Jurassic (Oxfordian and Late Callovian) Ammonites from the Western Interior Region of the United States

GEOLOGICAL SURVEY PROFESSIONAL PAPER 1232

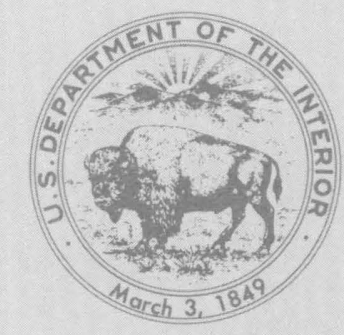




\section{Jurassic (Oxfordian and Late Callovian) Ammonites from the Western Interior Region of the United States}

By RALPH W. IMLAY

G E O L O I G A L S U R V E Y P R O F E S I O N A L P A P E R 1232

Marine waters invaded Montana in

late Callovian time,

advanced southward across Wyoming

in early Oxfordian time,

and persisted in Montana

during late Oxfordian time

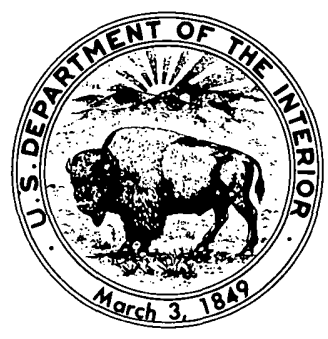

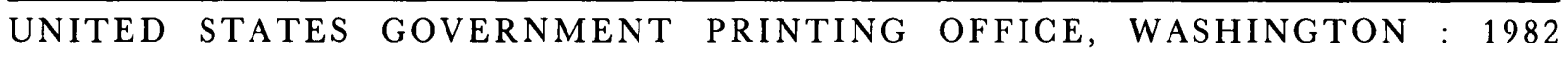




\section{UNITED STATES DEPARTMENT OF THE INTERIOR}

JAMES G. WATT, Secretary

\section{GEOLOGICAL SURVEY}

Dallas L. Peck, Director

\section{Library of Congress Cataloging in Publication Data}

Imlay, Ralph Willard, 1908-

Jurasic (Oxfordian and Late Callovian) ammonites from the western interior region of the United States.

(Geological Survey professional paper ; 1232)

Bibliography: $p$.

Includes index.

Supt. of Docs. no.: I 19.16:1232

1. Ammonidea. 2. Paleontology -Jurassic. 3. Paleontology - West (U.S.) I. Title. II. Series.

QE807.A51593 564'.53'0978 81-607015

AACR2

For sale by the Distribution Branch, U.S. Geological Survey, 604 South Pickett Street, Alexandria, VA 22304 


\section{CONTENTS}

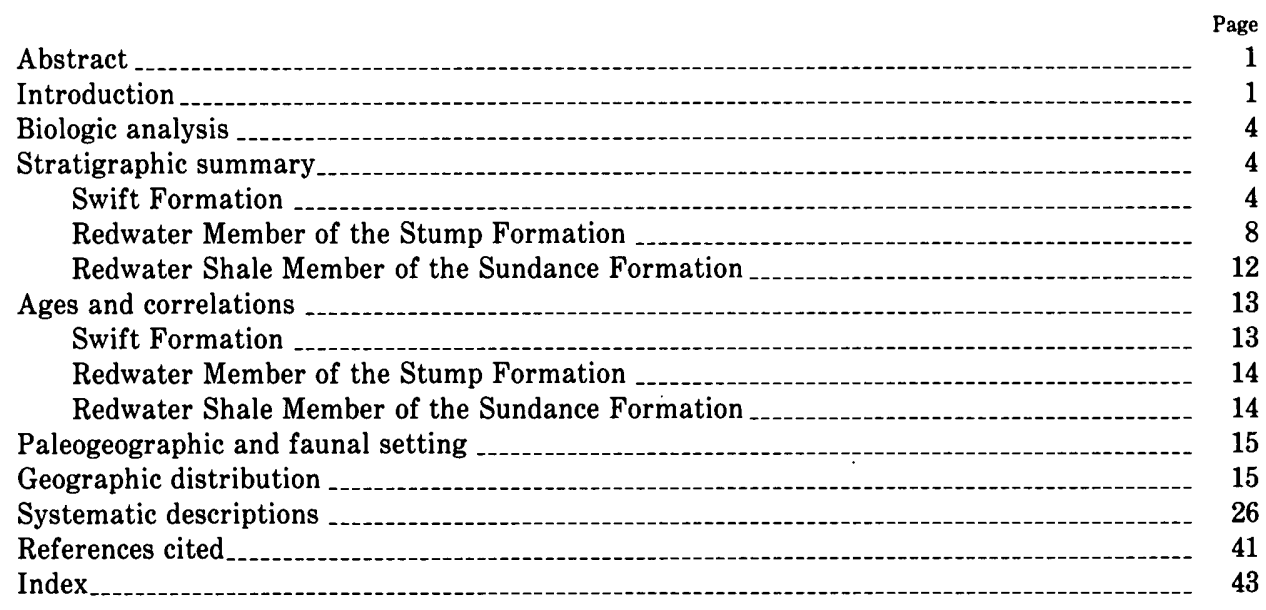

\section{ILLUSTRATIONS}

[Plates 1-26 follow index]

Plate 1. Quenstedtoceras (Lamberticeras) and Q. (Pavloviceras)

2. Quenstedtoceras (Pavloviceras) and Goliathiceras (Goliathiceras)

3. Goliathiceras (Goliathiceras)

4, 5. Goliathiceras (Goliathites)

6. Goliathiceras (Goliathites) and G. (Pachycardioceras)

7-13. Goliathiceras (Pachycardioceras)

14. Cardioceras (Scarburgiceras) and Goliathiceras (Pachycardioceras)

15. Cardioceras (Cardioceras)

16. Cardioceras (Scarburgiceras) and C. (Cardioceras)

17. Cardioceras (Cardioceras)

18-21. Cardioceras (Scarburgiceras)

22. Cardioceras (Scoticardioceras), C. (Cawtoniceras), and C. (Scarburgiceras)

23. Cardioceras (Maltoniceras)

24. Cardioceras (Maltoniceras?) and C. (Maltoniceras)

25. Cardioceras (Vertebriceras), Grossouvria?, and Grossouvria (Poculisphinctes?)

26. Cardioceras (Sagitticeras), Prososphinctes?, and Cardioceras (Subvertebriceras)

FIGURE 1. Generalized index map of latest Callovian and early to middle Oxfordian ammonite localities in the Western Interior region of the United States

2-6. Columnar sections of-

2. The Swift Formation in Montana

3. The Redwater Member of the Stump Formation in southeast Idaho and adjoining areas in Wyoming and Utah

4. The Redwater Shale Member of the Sundance Formation in the Bighorn Basin in north-central Wyoming

5. The Redwater Shale Member of the Sundance Formation in southeastern Wyoming

6. The Redwater Shale Member of the Sundance Formation in the Black Hills of northeastern Wyoming and western South Dakota.

7. Eurasian ranges of some late Callovian and Oxfordian ammonite genera and subgenera present in the

Western Interior region of the United States

8. Correlation of upper Callovian and Oxfordian marine formations in Montana and adjoining areas...........................

9. Correlation of Oxfordian marine formations in Wyoming and some adjoining States

10. Comparisons and correlation of late Callovian and Oxfordian ammonite faunas in the Western Interior region with similar faunas in western Canada and southern Alaska. 


\section{TABLES}

Table 1. Ammonite genera and subgenera of latest Callovian and early to middle Oxfordian Age in the Western Interior region of the United States

2. Geographic distribution of latest Callovian and Oxfordian ammonites in the Swift Formation in Montana

3. Geographic distribution of Oxfordian ammonites in the Redwater Member of the Stump Formation in southeastern Idaho and adjoining areas .

4. Geographic distribution of Oxfordian ammonites in the Redwater Shale Member of the Sundance Formation in the Bighorn and Wind River Basins of Wyoming

5. Geographic distribution of Oxfordian ammonites in the Redwater Shale Member of the Sundance Formation in central, south-central, east-central and southeastern Wyoming

6. Geographic distribution of Oxfordian ammonites in the Redwater Shale Member of the Sundance Formation in the Black Hills of northeastern Wyoming and western South Dakota

7. Description of Jurassic (late Callovian and Oxfordian) ammonite localities in the Western Interior region of the United States 


\title{
JURASSIC (OXFORDIAN AND LATE CALLOVIAN) AMMONITES FROM THE WESTERN INTERIOR REGION OF THE UNITED STATES
}

\author{
By RALPH W. IMLAY
}

\begin{abstract}
Ammonites collected at many localities in the north-central part of the Western Interior region show that during latest Callovian and most of Oxfordian time, the region was occupied by a sea that entered north-central and northeastern Montana from the northwest. That sea had strong connections northward with seas in Alaska and the Arctic region, as shown by the fact that most of the ammonites present are cardioceratids of boreal origin. It had weak connections westward, as shown by the presence of only three specimens of perisphinctid ammonites of Tethyan origin and by the absence of the ammonites Phylloceras and Lytoceras. Their absence in the Western Interior region contrasts with their abundance, in association with cardoceratid ammonites, in southern Alaska, where deposition took place near a major ocean in waters that were probably deeper than in an inland sea.

Ammonite age evidence indicates that the shallow sea that entered the Western Interior region during late Callovian time was restricted until earliest Oxfordian time to north-central Montana and to the Williston Basin farther east. In contrast, during late early Oxfordian time, the sea spread far southward across Wyoming and parts of adjoining States. Subsequently, during early middle Oxfordian time, the sea withdrew as far north as the south side of the Wind River Basin in central Wyoming and as far north as the Black Hills area in northeastern Wyoming and adjoining South Dakota. Then the sea advanced southward again as far as northern Colorado, deposited very shallow water sediments of slight thickness, but soon retreated northward into Montana during late middle Oxfordian time. It apparently persisted in the Williston Basin area as well as farther west
\end{abstract} until late Oxfordian time.

Ammonite evidence indicates that the Swift Formation in Montana ranges in age from latest Callovian to middle Oxfordian. The latest Callovian has been identified only in the Bearpaw and Little Rocky Mountains in north-central Montana by the presence of the subgenus Quenstedtoceras (Lamberticeras) in the lower 11-20 $\mathrm{m}$ of the lower shale member. Lamberticeras in the upper 1-2 $\mathrm{m}$ of its range was collected with Scarburgiceras, Pavloviceras, and Prososphinctes; this association in Europe is indicative of the upper part of the Quenstedtoceras lamberti zone. Nonetheless, some of the collections may represent mixtures from slightly different stratigraphic levels, or from float. This last possibility is favored by the fact that in both the Bearpaw Mountains and the Little Rocky Mountains, the highest occurrence of Lamberticeras is succeeded within 2 to $6 \mathrm{~m}$ by beds containing ammonites that correlate with the upper part of the Cardioceras cordatum zone, or with the lower part of the Persphinctes plicatilis zone.

The Cardioceras cordatum zone is represented by many ammonites in central and south-central Montana in the basal part of the Swift Formation. The zone of Perisphinctes plicatilis, however, may be represented at the southern margin of Glacier Park by an ammonite that in side view closely resembles Cardioceras (Scoticardioceras) whitfieldi Reeside and that is associated with many specimens of Buchia concentrica (Sowerby) at the top of the lower shale member. This occurrence shows that the overlying upper sandstone member must certainly be of middle Oxfordian Age and may in part be of late Oxfordian Age.

The Redwater Member of the Stump Formation in eastern Idaho and in nearby bordering States correlates in its lower and middle parts with the Cardioceras cordatum zone and probably is not younger than that zone.
The Redwater Shale Member of the Sundance Formation in central and southeastern Wyoming correlates entirely with the Cardioceras cordatum zone. That member in the Black Hills of northeastern Wyoming and western South Dakota is also correlated with that zone except possibly for its upper third, which has not furnished any ammonites. The lower shale unit of the same member in the Bighorn Basin is correlated with the $C$. cordatum zone. The upper sandstone unit of the Redwater Shale Member in that basin is probably in part of that age but could also be somewhat younger. The upper sandstone unit is herein correlated with the marine Windy Hill Sandstone Member south of the Bighorn Basin on the basis of their stratigraphic position conformably below the nonmarine Morrison Formation.

The fossil evidence shows that the Redwater Shale Member of the Sundance Formation is equivalent to only the lower part of the Swift Formation in Montana. It shows that the time represented by deposition of the upper sandstone member of the Swift Formation is represented in part in the Black Hills area and in southern Wyoming by an interval of erosion that was succeeded by deposition of the Windy Hill Sandstone Member of the Sundance Formation. The evidence suggests that marine deposition continued in parts of Montana while the lower part of the continental Morrison Formation was being deposited in areas to the south.

\section{INTRODUCTION}

The latest Jurassic ammonites from the Western Interior region of the United States (table 1) that were described by John B. Reeside, Jr., in 1919 have been restudied by the writer in order to (1) revaluate their geographic (fig. 1) and stratigraphic distribution (figs. 2-6) and the age significance of the genera and subgenera present (fig. 7); (2) make correlations with faunas of the same general ages in British Columbia and the Pacific Coast region as far north as Alaska (fig. 10); (3) date the formations more precisely in terms of the standard European zones (figs. 8-10); and (4) determine what changes took place in the distribution of land and sea.

This study is based on fossils from 159 localities, of which 52 are in the Swift Formation in Montana, 9 are in the Redwater Member of the Stump Formation in southeastern Idaho and adjoining states, 82 are in the Redwater Shale Member of the Sundance Formation in Wyoming, and 16 are in the same member in western South Dakota.

Field studies were made by the writer during parts of the summers of 1944, 1945, 1950, 1960, 1965, 1966, 1968, 1971-1973, and 1977. He was aided during parts of field seasons by W. A. Cobban in 1960 and 1972, N. F. Sohl in 1960, B. Carter Hearn in 1965, and G. N. Pipiringos in 1966 and 1977. Field assistants included H. C. Yingling in 1944, W. G. Saalfrank in 1945, and W. O. Ross in 1965, 1966,1968 , and 1971-1973. 
JURASSIC (OXFORDIAN, LATE CALLOVIAN) AMMONITES, WESTERN INTERIOR, UNITED STATES

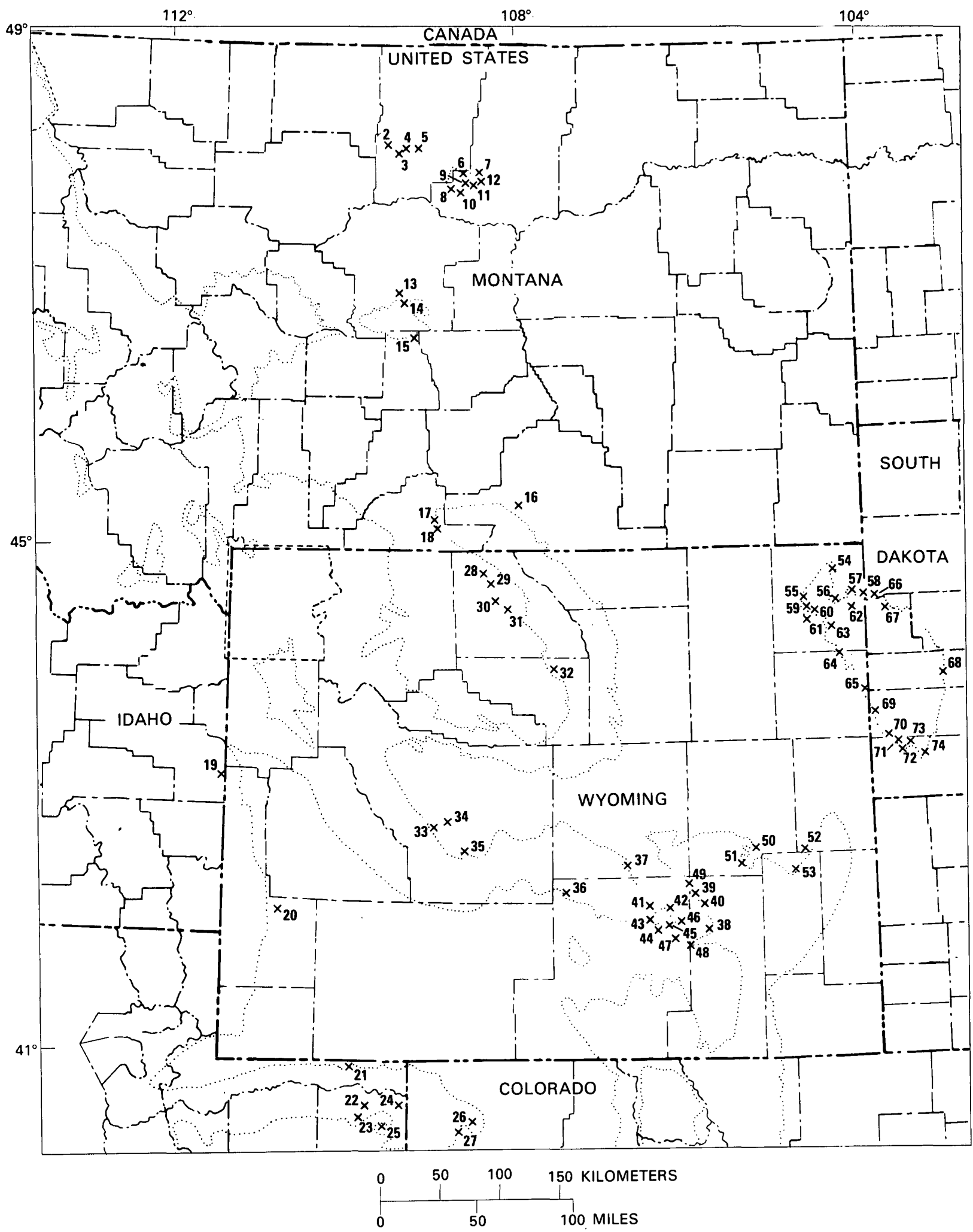

FIGURE 1.-Latest Callovian and early to middle Oxfordian ammonite localities in the Western Interior region of the United States. Dotted lines represent boundaries of mountain ranges. 


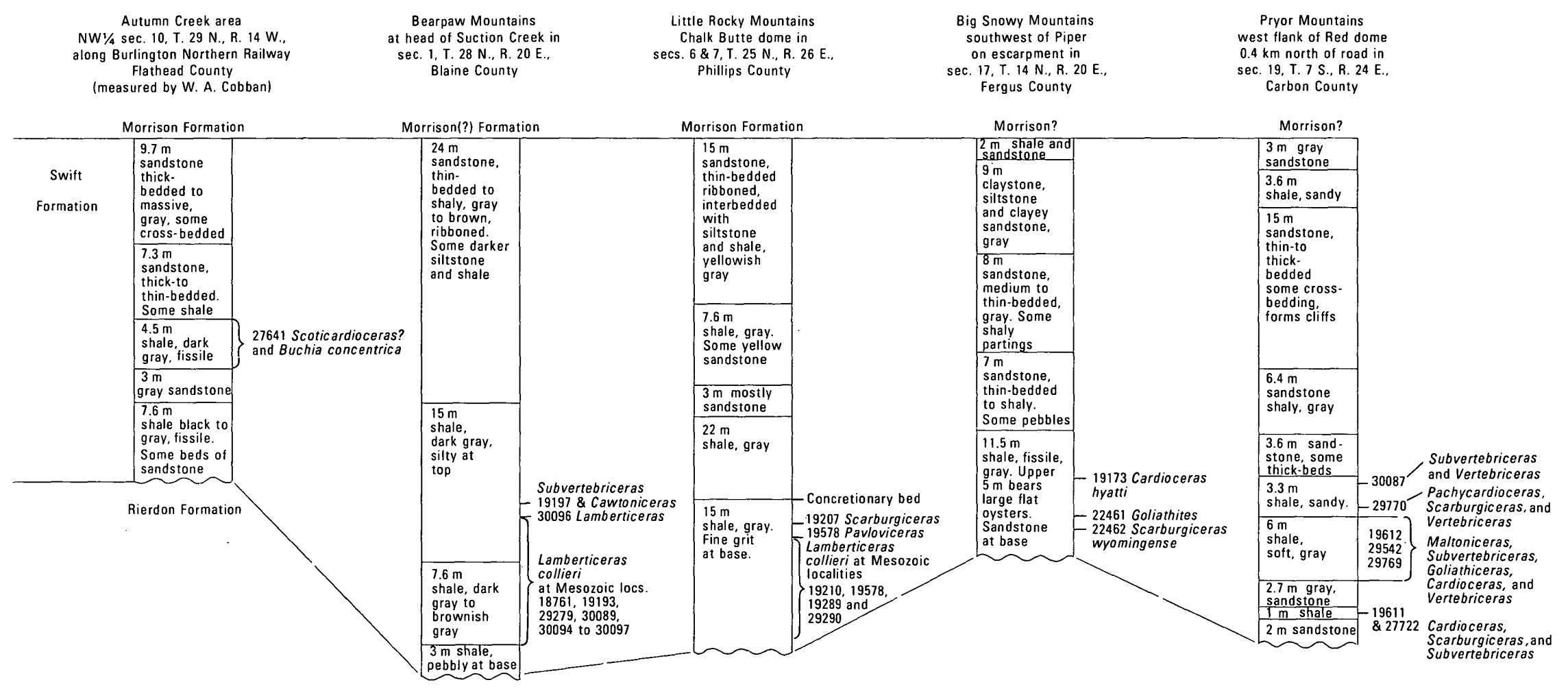

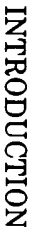

FIGURE 2-Columnar sections of the Swift Formation in Montana. List of species by localities is given in table 2. 


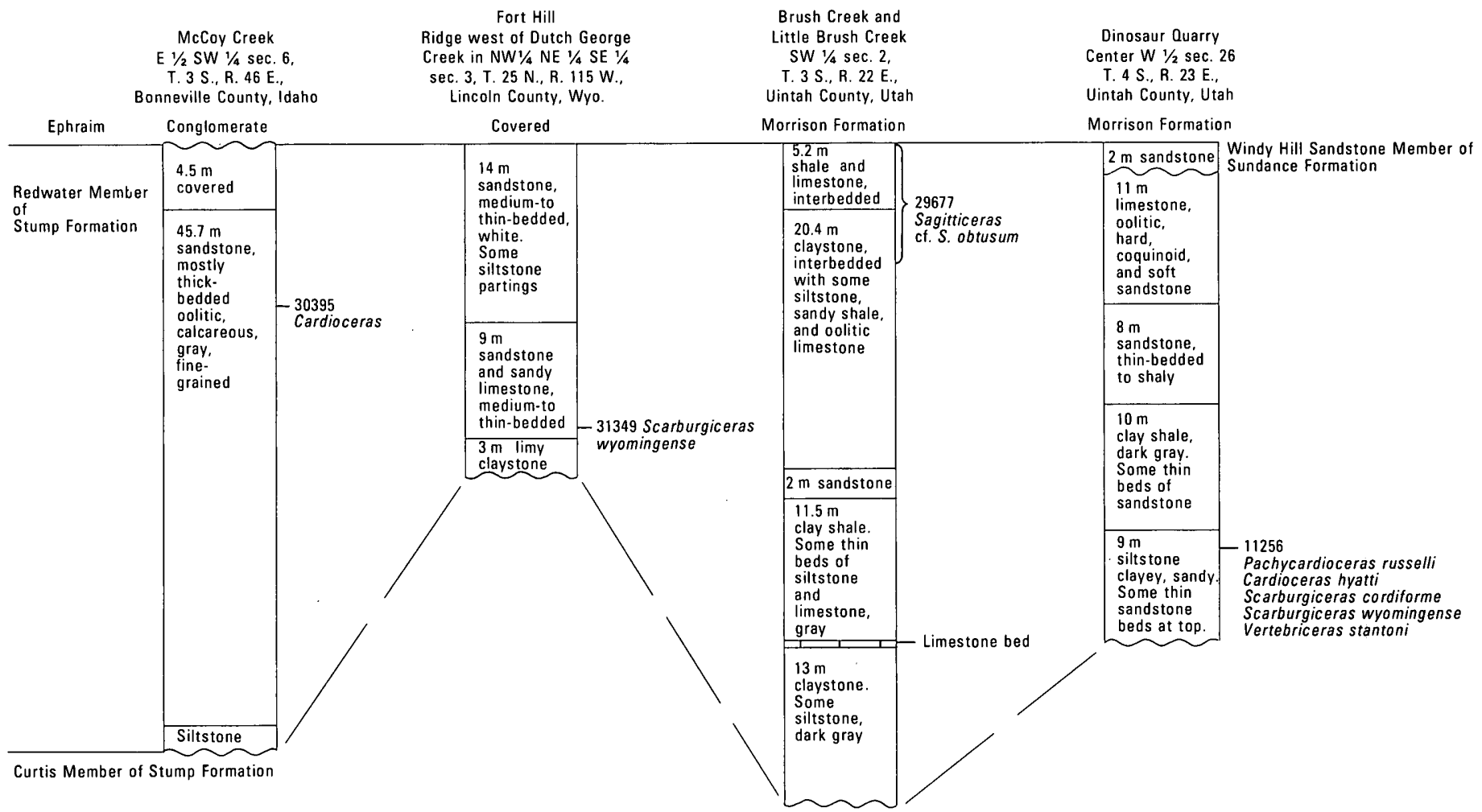

FIGURE 3.-Columnar sections of the Redwater Member of the Stump Formation in southeastern Idaho and adjoining areas in Wyoming and Utah. List of species by localities is given in table 3.

\section{BIOLOGIC ANALYSIS}

The ammonites of latest Callovian to early middle Oxfordian Age that are described herein number 885 specimens. Their distribution by family, subfamily, genus, and subgenus is shown on table 1. Only two families are represented. Of these, the Perisphinctidae are represented by three specimens and the Cardioceratidae by the rest. Among the dominant subgenera present, Lamberticeras includes 34 percent of the total number of specimens; Vertebriceras, 15 percent; Subvertebriceras, about 12 percent; Cardioceras, about 11 percent; Scarburgiceras, 7 percent; Cawtoniceras, about 5 percent; Pachycardioceras, 4 percent; and Pavloviceras, about 3 percent.

\section{STRATIGRAPHIC SUMMARY}

\section{SWIFT FORMATION}

The Swift Formation in Montana (fig. 2) ranges in thickness from about 1 to $50 \mathrm{~m}$ in surface outcrops and to $122 \mathrm{~m}$ or more in the subsurface; consists of glauconitic sandstone, siltstone and claystone; and generally has a basal conglomerate in surface sequences. The Swift is underlain unconformably by the Rierdon Formation and is overlain conformably by the Morrison Formation, which thins northward and disappears near the Canadian border.

In northern Montana, the Swift Formation consists of two transitional members of similar thickness. The upper sandstone member consists mostly of thin- to thick-bedded glauconitic, ripple-marked sandstone but includes some micaceous shale partings. The lower shale member consists mostly of dark, fissile, micaceous noncalcareous shale. Eastward from the Sawtooth Range, west of Great Falls, these members pinch out on the Sweetgrass arch but reappear farther east and become shalier eastward (Cobban, 1945, p. 1281-1286; Imlay and others 1948).

TABLE 1.-Ammonite genera and subgenera of latest Callovian and early to middle Oxfordian Age in the Western Interior region of the United States

\begin{tabular}{|c|c|c|c|}
\hline Family & Subfamily & $\begin{array}{l}\text { Genus and } \\
\text { subgenus }\end{array}$ & $\begin{array}{l}\text { No. of } \\
\text { specimens }\end{array}$ \\
\hline \multirow[t]{15}{*}{ Cardioceratidae } & Cardioceratinae & Quenstedtoceras & \\
\hline & & (Lamberticeras) & 300 \\
\hline & & (Pavloviceras) & 29 \\
\hline & & Goliathiceras & 16 \\
\hline & & (Goliathites) & 15 \\
\hline & & (Pachycardioceras) & 34 \\
\hline & & Cardioceras & 100 \\
\hline & & (Scarburgiceras) & 71 \\
\hline & & (Scoticardioceras) & 6 \\
\hline & & (Scoticardioceras?) & 1 \\
\hline & & (Cawtomiceras) & 40 \\
\hline & & (Maltoniceras) & 22 \\
\hline & & (Subvertebriceras) & 110 \\
\hline & & (Vertebriceras) & 132 \\
\hline & & (Sagitticeras) & 6 \\
\hline \multirow[t]{3}{*}{ Perisphinctidae } & $\begin{array}{l}\text { Pseudoperi- } \\
\text { sphinctinae }\end{array}$ & Grossouvria? & 1 \\
\hline & & (Poculisphinctes?) & 1 \\
\hline & Perisphinctinae & Prososphinctes? & 1 \\
\hline
\end{tabular}




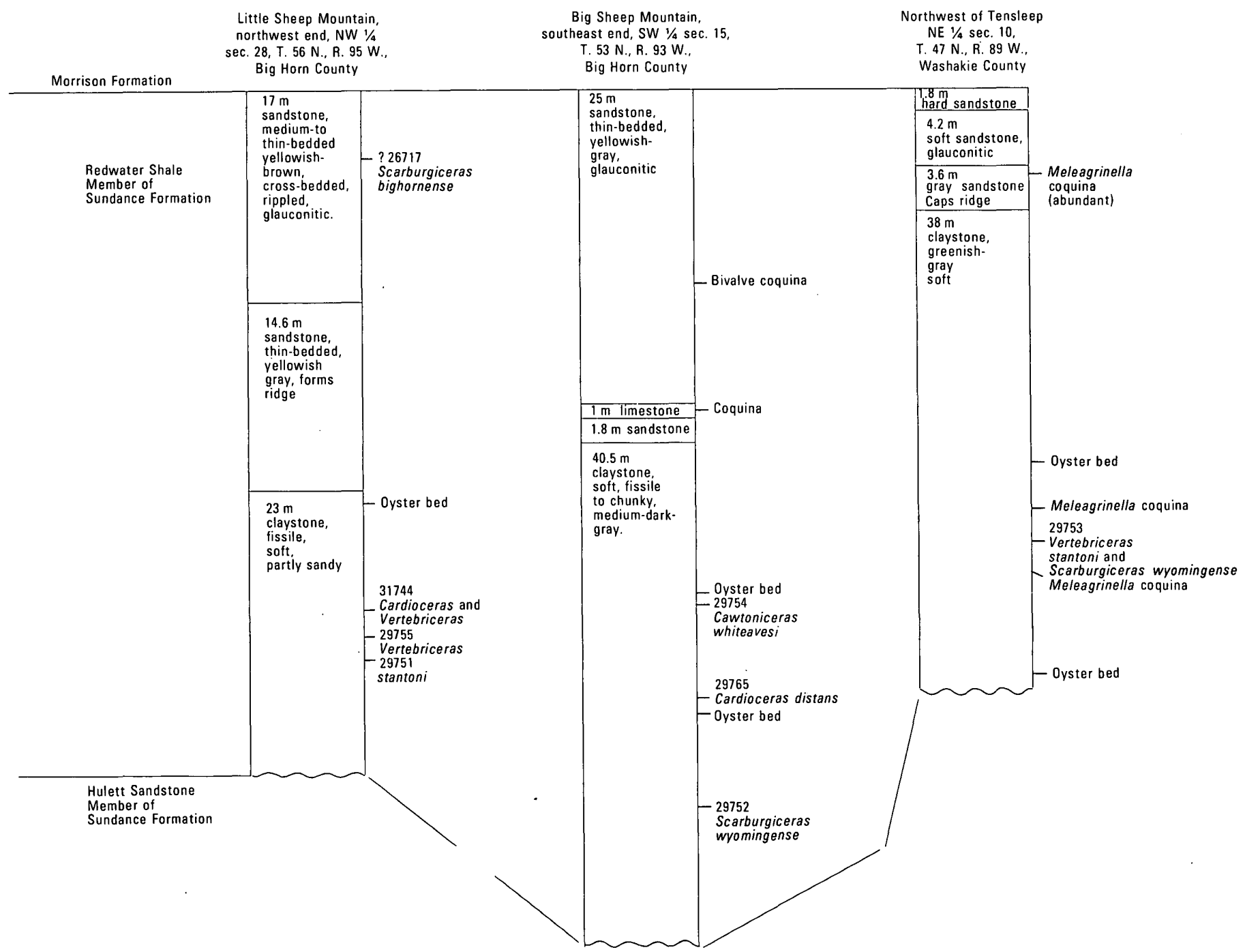

FIGURE 4.-Columnar sections of the Redwater Shale Member of the Sundance Formation in the Bighorn Basin in north-central Wyoming. List of species by localities is given in table 4 .

In southwestern and central Montana, by contrast, the Swift Formation consists mostly of medium- to thick-bedded glauconitic ripple-marked sandstone, but some shale appears at or near the base in the Big Snowy and Pryor Mountains of Central and south-central Montana and thickens eastward (Imlay and others; 1948; Imlay, 1954, 1956, p. 566; Moritz, 1951, p. 1804-1810). These stratigraphic relationships and lithologic changes have been summarized by Imlay $(1980$, p. 68,82 , 113,114 ).

The lithologic features of the Swift Formation at many localities in the Sweetgrass arch area have been described by Cobban (1945, p. 1291-1303), in southwestern Montana by Moritz (1951, p. 1805-1810), and in many parts of Montana by Imlay and others (1948), by Imlay $(1954$, p. $61-64 ; 1956$, p. 566$)$, and by Mudge (1972, p. A97-A106). In addition, a description of the section of the Swift Formation measured by W. A. Cobban in the
Autumn Creek area south of Glacier Park is presented herein because of its age significance.

Swift Formation exposed a little east of Autumn Creek in NW1/4 sec. 10, T. $29 \mathrm{~N}, \mathrm{R} .14 \mathrm{~W}$., Flathead County, Mont.

Morrison Formation

Thickness (Meters)

Swift Formation:

Sandstone, thick-bedded to massive, ripple-marked, glauconitic

Sandstone, thin- to medium-bedded, some interbedded sandy shale, light-gray

Claystone, fissile, micaceous, noncalcareous, dark-gray to black; contains Buchia concentrica (Sowerby) in abundance

Sandstone, thick-bedded, light-gray

3.0

Shale, silty to sandy, dark-gray 7.6 Total thickness 32.1 


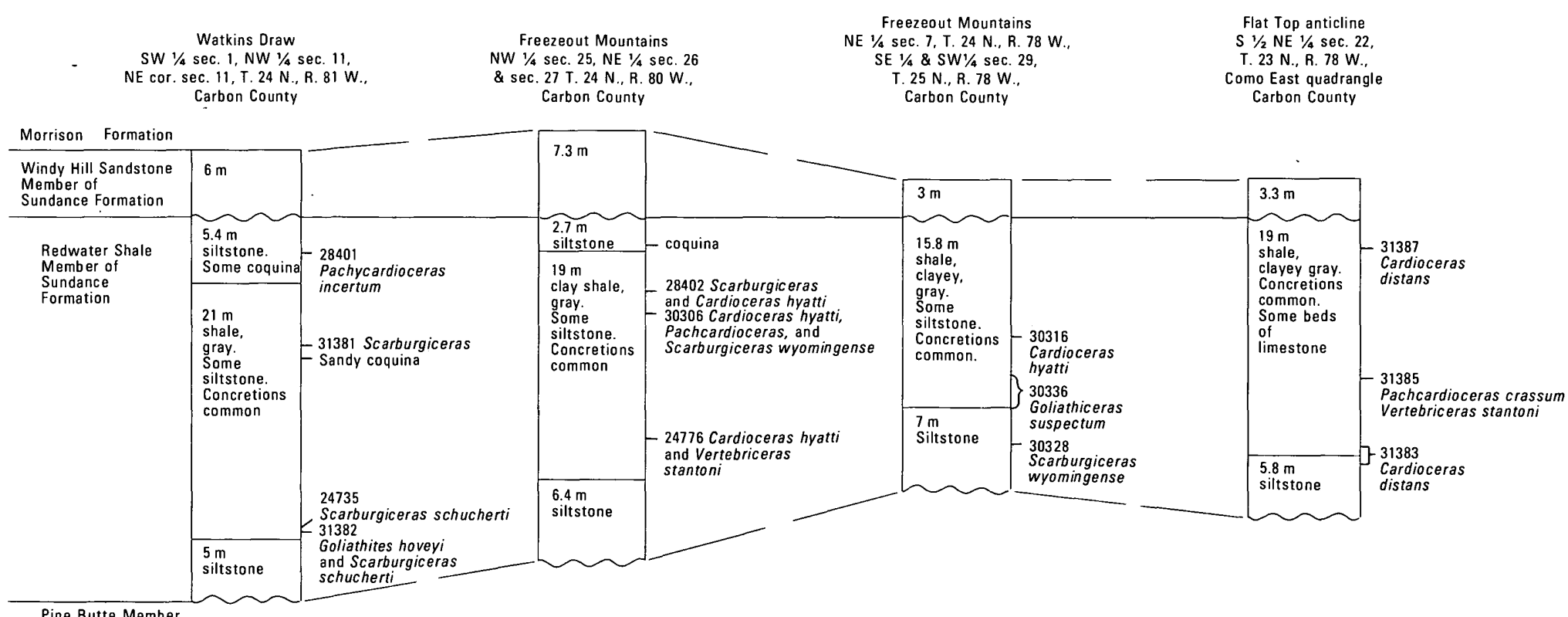

Pine Butte Member
of Sundance Formation

FIGURE 5.-Columnar sections of the Redwater Shale Member of the Sundance Formation in southeastern Wyoming. List of species by localities is given in table 5. 


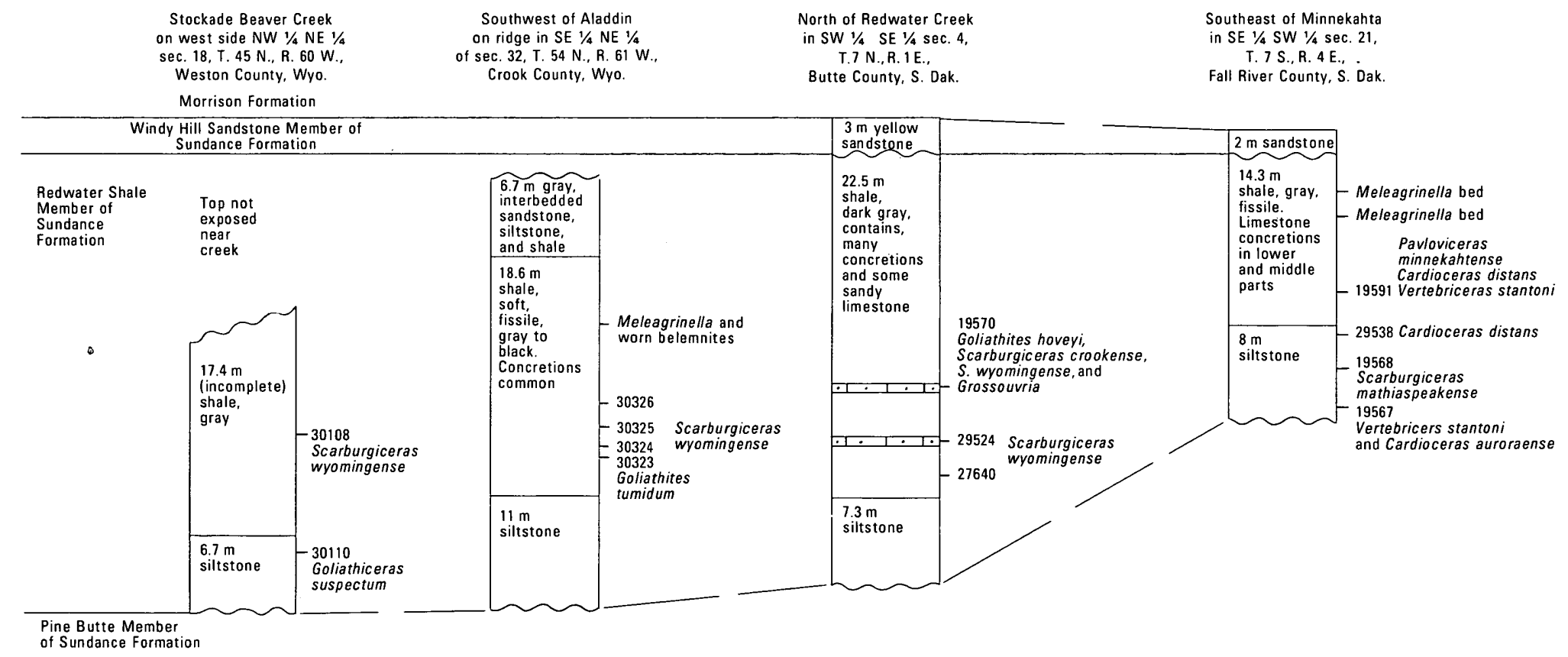

FIGURE 6.-Columnar sections of the Redwater Shale Member of the Sundance Formation in the Black Hills of northeastern Wyoming and western South Dakota. List of species by localities is given in table 6 . 


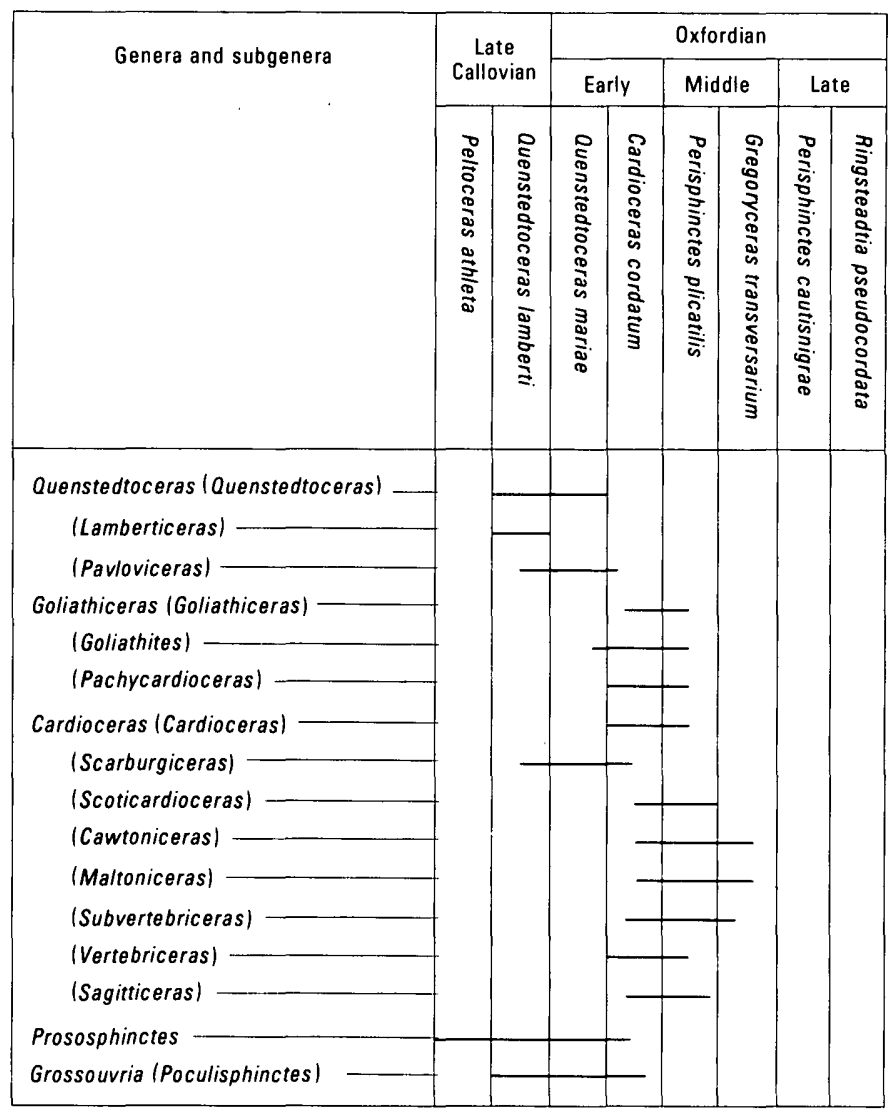

FIgURE 7.-Eurasian ranges of some late Callovian and Oxfordian ammonite genera and subgenera present in the Western Interior region of the United States.

\section{REDWATER MEMBER OF THE STUMP FORMATION}

The Redwater Member of the Stump Formation (fig. 3 ) crops out in (1) southeastern Idaho in the mountains bordering the Snake River, (2) western Wyoming in the Wyoming Range, (3) the southwestern corner of Wyoming near Evanston, (4) northeastern Utah from Peoa eastward, and (5) the northwestern corner of Colorado (Pipiringos and Imlay, 1979, fig. 3 on p. C6). The Redwater ranges in thickness from a featheredge to about $55 \mathrm{~m}$, consists mostly of thin- to thick-bedded, calcareous, glauconitic sandstone interbedded with some siltstone and silty sandstone, and is locally oolitic, crossbedded, or ripplemarked. This sandstone unit is underlain at McCoy Creek in southeastern Idaho and in the Wyoming Range of western Wyoming by a thin shale unit that ranges from 1.8 to $5.6 \mathrm{~m}$ in thickness and consists of glauconitic siltstone and claystone that locally is sandy. This shale unit becomes nearly twice as thick farther south near Evanston, Wyo., and near Peoa, Utah. From Peoa eastward, it thickens to about $18 \mathrm{~m}$ at Brush Creek and to $19 \mathrm{~m}$ near Dinosaur Quarry. Both units contain a fair abundance of belemnites and bivalves but very few ammonites, except for one occurrence in the lower shale unit near Dinosaur Quarry in northeastern Utah (fig. 3). (For further details concerning sections, see Pipiringos and Imlay, 1979, p. C17-C25.)

The Redwater Member of the Stump Formation rests unconformably on the Curtis Member but does not extend nearly as far south or southwest. It is overlain unconformably by the Ephraim Conglomerate from near Peoa northward to westernmost Wyoming and southeastern Idaho. The Redwater is overlain conformably by the Morrison Formation in the Uinta Mountains from a little east of Peoa to a little east of Vernal in northeast Utah. Still farther east near Dinosaur Quarry, the Redwater is overlain unconformably by the Windy Hill Sandstone Member of the Sundance Formation, which in turn is overlain conformably by the Morrison Formation. Typically, the Redwater Member of the Stump, as exposed in southeastern Idaho and westernmost Wyoming, differs from the Redwater Shale Member of the Sundance Formation, as exposed throughout most of Wyoming, by being sandier and by having a much thinner basal lower shale unit, or none at all. Its sequences, as exposed in the eastern part of the Uinta Mountains, and in an adjoining part of northwest Colorado, show that the Redwater Member represents a western extension of the Redwater Shale Member. 


\begin{tabular}{|c|c|c|c|c|c|c|c|c|c|}
\hline \multicolumn{3}{|r|}{ Zones in northwest Europe } & \multicolumn{2}{|c|}{$\begin{array}{l}\text { Autumn Creek at } \\
\text { south edge of } \\
\text { Glacier National Park in } \\
\text { northwest Montana }\end{array}$} & $\begin{array}{l}\text { Little Rocky } \\
\text { Mountains } \\
\text { in north-central } \\
\text { Montana }\end{array}$ & $\begin{array}{l}\text { Big Snowy Mountains } \\
\text { southwest of Piper } \\
\text { in central Montana }\end{array}$ & \multicolumn{2}{|r|}{$\begin{array}{l}\text { Pryor Mountains } \\
\text { at Red dome in } \\
\text { south-central } \\
\text { Montana }\end{array}$} & $\begin{array}{c}\text { Bighorn Basin } \\
\text { in north-central } \\
\text { Wyoming }\end{array}$ \\
\hline \multirow{5}{*}{ 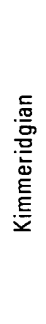 } & \multirow{3}{*}{ 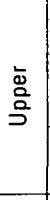 } & Aulacostephanoceras acutissiodorenses & \multirow{7}{*}{\multicolumn{2}{|c|}{$\begin{array}{l}\text { Morrison } \\
\text { Formation } \\
12 \mathrm{~m}\end{array}$}} & \multirow{7}{*}{$\begin{array}{c}\text { Morrison } \\
\text { Formation } \\
20-25 \mathrm{~m}\end{array}$} & \multirow{7}{*}{$\begin{array}{l}\text { Morrison Formation } \\
\text { (lower part) } \\
\text { Total thickness } \\
67 \mathrm{~m}\end{array}$} & \multirow{8}{*}{\multicolumn{2}{|c|}{$\begin{array}{c}\text { Morrison } \\
\text { Formation } \\
\text { (lower part) } \\
52 \mathrm{~m}\end{array}$}} & \multirow{9}{*}{$\begin{array}{c}\text { Morrison } \\
\text { Formation } \\
55-79 \mathrm{~m}\end{array}$} \\
\hline & & Aulacostephanus eudoxus & & & & & & & \\
\hline & & Rasenia mutabilis & & & & & & & \\
\hline & \multirow{2}{*}{ 离 } & Rasenia cymodoce & & & & & & & \\
\hline & & Pictonia baylei & & & & & & & \\
\hline \multirow{7}{*}{ 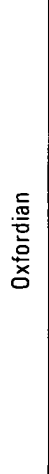 } & \multirow{3}{*}{$\begin{array}{l}\text { ฏ } \\
\text { 음 }\end{array}$} & Ringsteadtia pseudocordata & & & & & & & \\
\hline & & Decipia decipiens & & & & & & & \\
\hline & & Perisphinctes cautisnigrae & \multirow{10}{*}{ 䒺高 } & \multirow{2}{*}{$\begin{array}{c}\text { Upper } \\
\text { sandstone member } \\
17 \mathrm{~m}\end{array}$} & \multirow{2}{*}{$\begin{array}{c}\text { Upper } \\
\text { sandstone member } \\
18-24 \mathrm{~m}\end{array}$} & Upper & & & \\
\hline & \multirow{2}{*}{$\begin{array}{l}\stackrel{0}{\bar{z}} \\
\stackrel{\bar{z}}{\Sigma}\end{array}$} & Gregoryceras transversarium & & & & $7-26 \mathrm{~m}$ & & Upper & \\
\hline & & Perisphinctes plicatilis & & $\begin{array}{l}\text { Lower shale member } \\
14 \mathrm{~m}\end{array}$ & $\begin{array}{l}\text { Lower } \\
\text { shale }\end{array}$ & $\begin{array}{l}\text { Lower } \\
\text { shale land }\end{array}$ & 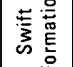 & $32 \mathrm{~m}$ & $\begin{array}{l}\text { Redwater Shale } \\
\text { Member }\end{array}$ \\
\hline & $\bar{\Phi}$ & Cardioceras cordatum & & & $\begin{array}{l}\text { member } \\
27-30 \mathrm{~m}\end{array}$ & $\begin{array}{l}\text { member } \\
11.5-13 \mathrm{~m}\end{array}$ & & $\begin{array}{c}\text { Lower shale member } \\
15.5 \mathrm{~m}\end{array}$ & \\
\hline & 9 & Quenstedtoceras mariae & & & & & & & \\
\hline \multirow{6}{*}{ 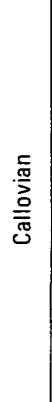 } & \multirow{2}{*}{ 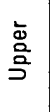 } & Quenstedtoceras lamberti & & & & & & & $\subseteq$ \\
\hline & & Peltoceras athleta & & & & & & & E⿱⿱㇒⿴囗夊心 \\
\hline & \multirow{2}{*}{$\frac{\frac{0}{0}}{\frac{\pi}{\bar{\nu}}}$} & Erymnoceras coronatum & & & & & & & 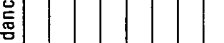 \\
\hline & & Kosmoceras jason & & & & & & & \\
\hline & \multirow{2}{*}{$\sum_{0}^{\circ}$} & Sigaloceras calloviense & & & & & & & \\
\hline & & Macrocephalites macrocephalus & \multicolumn{2}{|r|}{$\begin{array}{l}\text { Rierdon Formation } \\
\text { (upper part) }\end{array}$} & $\begin{array}{l}\text { Rierdon Formation } \\
\text { (upper part) }\end{array}$ & $\begin{array}{l}\text { Rierdon Formation } \\
\text { (upper part) }\end{array}$ & & $\begin{array}{l}\text { Sierdon Formation } \\
\text { (upper part) }\end{array}$ & $\begin{array}{l}\text { Hulett Sandstone } \\
\text { Member } 5-32 \mathrm{~m}\end{array}$ \\
\hline
\end{tabular}

FIGURE 8.-Correlation of upper Callovian and Oxfordian marine formations in Montana and adjoining areas. 


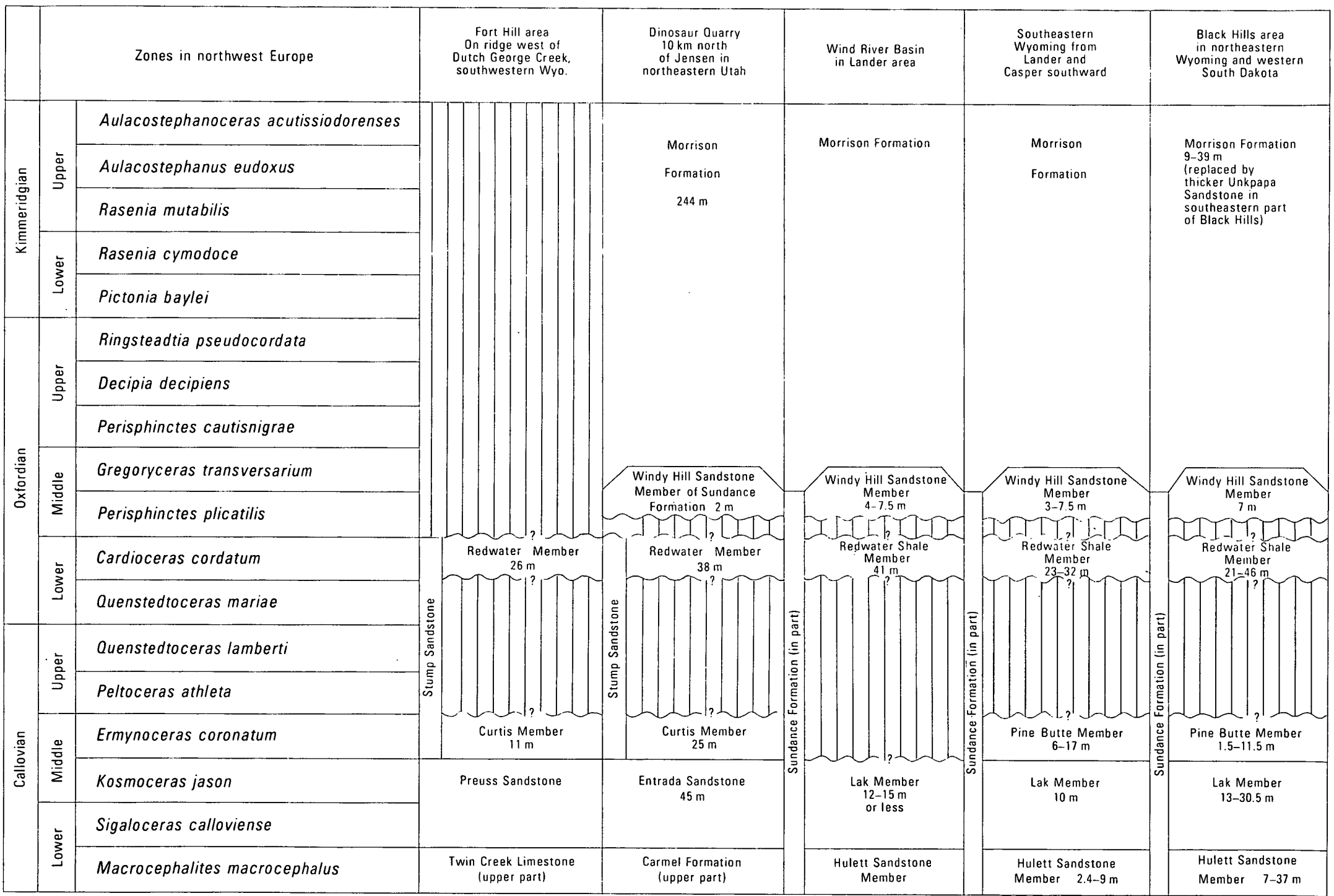

FIGURE 9.-Correlation of Oxfordian marine formations in Wyoming and some adjoining States. 


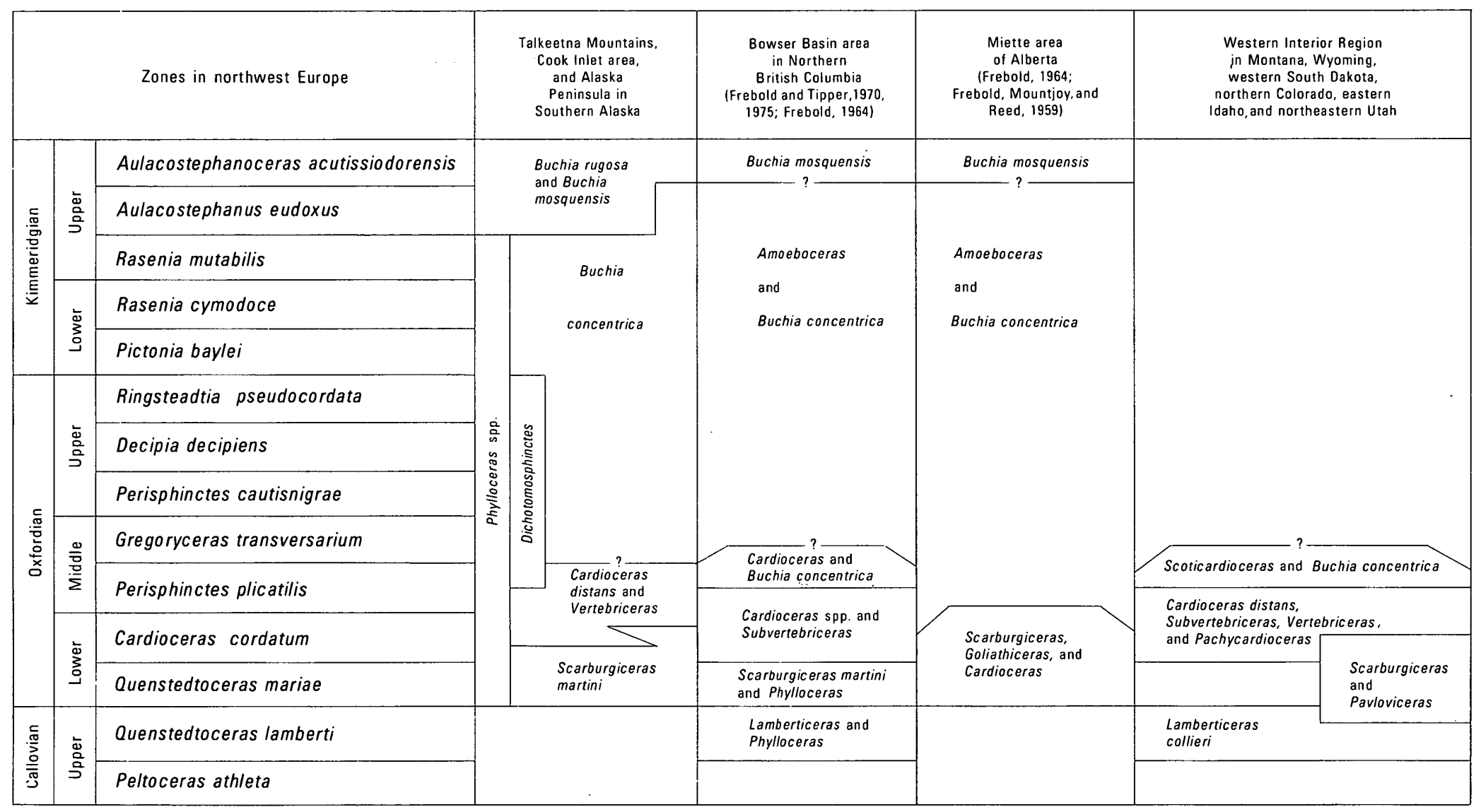

FIGURE 10-Comparisons and correlation of late Callovian and Oxfordian ammonite faunas in the Western Interior region with similar faunas in western Canada and southern Alaska. 
REDWATER SHALE MEMBER OF THE SUNDANCE FORMATION

In the Bighorn Basin of north-central Wyoming (fig. 4), the Redwater Shale Member of the Sundance Formation ranges in thickness from 37 to $53 \mathrm{~m}$ on the west side of the basin and from 44 to $76 \mathrm{~m}$ on the east side and south end of the basin (Imlay, 1956, p. 567-571, $595,598)$. Within the basin, the member thickens irregularly southward except near Tensleep, where thinning took place during Oxfordian time along an axis of a southward-trending uplift, which is probably an extension of the Sheridan arch (Peterson, 1954, p. 474-477, $503 ; 1957$, p. 403-405).

The Redwater Shale Member on the east side of the basin consists of a lower shale unit and an upper sandstone unit. The lower shale unit consists of soft, fissile, calcareous claystone interbedded with silty to sandy beds, which grades upward into the sandstone unit and which rests unconformably on the Hulett Sandstone Member of the Sundance Formation. The sandstone unit consists of thin- to thick-bedded, glauconitic sandstone interbedded with some shaly sandstone. It constitutes more than half the total member at the northern end of the east side of the basin near Lovell; a little less than half, farther south near Greybull; and only a fourth or less, from Tensleep southward. The member is overlain sharply but apparently conformably by the continental Morrison Formation. (For further detail concerning local sections, see Imlay, 1956, p. 567-573, 595-598.)

In contrast, the Redwater Shale Member of the Sundance on the west side of the Bighorn Basin consists mostly of glauconitic, thin- to thick-bedded, partly crossbedded, partly cliff-forming sandstone. Below that is 19-21 $\mathrm{m}$ of softer thin-bedded sandstone interbedded with silty sandstone. At the base, as exposed in the gorge of the Shoshone River, is about $1 \mathrm{~m}$ of silty claystone that contains pebbles of limestone and chert and that rests on about $5 \mathrm{~m}$ of sandy beds representing the westernmost featheredge of the Hulett Sandstone Member of the Sundance Formation (Imlay, 1956, p. 592). All facies contain an abundance of belemnites.

These lithologic and stratigraphic features show that the Redwater Shale Member of the Sundance in the Bighorn Basin closely resembles the Swift Formation in the Pryor Mountains (Imlay, 1954, p. 55, 57) in southcentral Montana and was deposited under essentially the same conditions. In both areas, those named lithologic units (1) become thinner and sandier westward, (2) are overlain sharply but conformably by the Morrison Formation, (3) rest unconformably on the Hulett Sandstone Member of the Sundance in the easternmost exposures, and (4) rest unconformably on calcareous shales in their westernmost exposures near Cody, Wyo., and at Red dome at the west end of the Pryor Mountains. These facts show that the history of sedimentation during Late Jurassic time was essentially the same throughout the area comprising the Bighorn Basin and the adjoining mountains to the east and northeast.

In central, south-central, and southeastern Wyoming (fig. 5), the Redwater Shale Member of the Sundance differs from that in the Bighorn Basin (1) by being overlain unconformably by the marine Windy Hill Sandstone Member of the Sundance Formation; (2) by lying unconformably at most places on the Pine Butte or the Lak Member of the Sundance rather than on the Hulett Sandstone Member of the Sundance; (3) by becoming much shalier to the east and southeast; and (4) by being divisible in south-central and southeastern Wyoming into four units, which from bottom to top are alternately siltstone and shale (Pipiringos, 1968, p. D4, D23). Toward the northwest, about $32 \mathrm{~km}$ southeast of Lander in the Wind River Basin, the upper units pass into shelly limestone and glauconitic cliff-forming sandstone, and the lower two units pass into softer, silty, or clayey sandstone. Toward the east in south-central Wyoming, the upper two units are beveled out in the Freezeout Mountains by an unconformity at the base of the Windy Hill Sandstone Member (Pipiringos, 1968, fig. 3 on p. D4). Similarly, in southeastern Wyoming, all four units are beveled out southward from Douglas by the same unconformity (Pipiringos and O'Sullivan, 1976). (For details concerning local sections, see Love and others, 1945; Love, 1958; Pipiringos, 1957, 1968; and Pipiringos and O'Sullivan, 1978.)

The Redwater Shale Member of the Sundance in the Black Hills (fig. 6) in eastern Wyoming and western South Dakota differs from that member in southcentral and east-central Wyoming by consisting of only two units. Of these, the lower unit consists mostly of siltstone but locally contains some sandy beds. Its thickness ranges from $4.5 \mathrm{~m}$ near Buffalo Gap, S. Dak., to $11 \mathrm{~m}$ near Aladdin, Wyo., but at most places it is about $8 \mathrm{~m}$. The upper unit consists mostly of greenish-gray shale but also contains some thin beds of coquinoidal, or oolitic, or sandy limestone in its upper half and at a few localities contains some fine-grained glauconitic sandstone. Its thickness ranges from 1.5 to $30 \mathrm{~m}$. These two units are similar lithologically to the two units present in the Freezout Mountains, East, as illustrated by Pipiringos $(1968$, p. D8, D12). Possibly some of the silty to sandy beds near the top of the Redwater Shale Member, as exposed near Aladdin and north of Spearfish, could be equivalent to the upper siltstone unit as described by Pipiringos (1968, p. D12). The Redwater Shale Member in the Black Hills contains belemnites and bivalves throughout, but ammonites have been found only in its lower and middle parts.

The lithologic features of the Redwater Shale Member at many localities in the Black Hills have been described by Imlay (1947, p. 245, 266-273), Cuppels (1963, p. 283), Gott and Schnabel (1963, p. 143), Mapel and Pillmore (1963, p. M12), and Post (1967, p. 453). In 
addition, a section measured in a fresh roadcut across a ridge southwest of Aladdin (fig. 6) has furnished some fossils of age significance and is presented herein.

Redwater Shale Member of Sundance Formation on ridge southwest of Aladdin in SE1/4 NE1/4 sec. 32, T. 54 N., R. 61 W., Crook County, Wyo. Thickness

Redwater Shale Member:

23. Interbedded sandstone, siltstone, and a little claystone, yellowish-gray

22. Siltstone, shale, and thin beds of sandstone

21. Shale, black, fissile, soft

20. Sandstone, yellowish-gray, and limestone pebbles

19. Shale

18. Concretionary bed

17. Shale

16. Concretionary bed

15. Shale, gray, fissile, soft

14. Concretionary bed (USGS Mesozoic loc. 30326)

13. Shale

12. Concretionary bed

11. Shale

10. Concretionary bed (USGS Mesozoic loc. 30325)

9. Shale, gray, fissile, soft

8. Concretionary bed (USGS Mesozoic loc. 30324)

7. Shale, gray, fissile, soft

6. Concretionary bed. (USGS Mesozoic loc. 30323)

5. Shale, gray, fissile, soft

4. Claystone

3. Alternating beds of yellowish silty sandstone, sandy siltstone, and silty shale; worn belemnites at upper contact.

2. Silty shale, gray

1. Sandy siltstone, highly glauconitic crossbedded; contains worn belemnites; rests sharply on Lak Member of Sundance

Total thickness

\section{AGES AND CORRELATIONS}

\section{SWIFT FORMATION}

The age of the Swift Formation in Montana (figs. 7, 8), on the basis of ammonites, ranges from latest Callovian, as now defined in western Europe (Callomon, 1964, p. 284, 289; Enay and others, 1971, p. 652,661), to early middle Oxfordian. Its basal beds, however, vary considerably in age from place to place, and its upper beds, which have not yielded any ammonites, could be in part of late Oxfordian Age.

The oldest beds in the Swift Formation are characterized by the subgenus Quenstedtoceras (Lamberticeras), which in Europe occurs only in the zone of Quenstedstoceras lamberti. That subgenus to date has been found in the Western Interior region only in the Bearpaw and Little Rocky Mountains of north-central Montana. In those mountains, Lamberticeras occurs in the lower $10.6-18 \mathrm{~m}$ of a claystone-siltstone unit in the lowest part of the Swift Formation exposed around the margins of structural domes (Knechtel, 1944). That subgenus in the Little Rocky Mountains is associated at a few places in the upper part of its range with several other ammonite taxa. Thus, at Chalk Butte dome, Lamberticeras in the uppermost meter of its range is associated with Scarburgiceras (USGS Mesozoic loc. 9207) and Pavloviceras (USGS Mesozoic loc. 19578). At Morrison dome, Lamberticeras is associated with the same taxa in shale just below the base of the upper sandstone unit. At Camp Creek dome, Lamberticeras is associated with Scarburgiceras and Prososphinctes (USGS Mesozoic loc. 19579) from 6 to $7.6 \mathrm{~m}$ above the base of the Swift Formation, but it ranges as much as $18 \mathrm{~m}$ above the base. Judging by the ranges of these genera in Europe, the beds containing an association of Lamberticeras, Pavloviceras, and Scarburgiceras should correlate mainly with the upper part of the Quenstedtoceras lamberti zone.

Ammonites indicative of the west European zone of Quenstedtoceras mariae have not yet been found in Montana, and only a slight thickness of beds in the Bearpaw Mountains could belong to that zone. Thus, at Taylor Butte in the Bearpaw Mountains, an occurrence of Lamberticeras $10.6 \mathrm{~m}$ above the base of the Swift Formation is succeeded $4.5 \mathrm{~m}$ higher by a faunule (USGS Mesozoic loc. 29278) consisting of Cardioceras $\mathrm{cf}$. C. lilloetense Reeside, C. (Cawtoniceras) whiteavesi Reeside, C. (Maltoniceras) sundancense Reeside, and $C$. (Vertebriceras) stantoni Reeside, which are indicative of the upper part of the Cardioceras cordatum zone or the lower part of the Peisphinctes plicatilis zone of Europe. Similarly, at Suction Creek in the Bearpaw Mountains, a collection made only about $1 \mathrm{~m}$ above the highest occurrence of Lamberticeras contains the ammonites $C$. (Subvertebriceras) canadense Whiteaves and C. (Cawtoniceras) whiteavesi Reeside of late $C$. cordatum or early P. plicatilis age (USGS Mesozoic loc. 19197). Failure to find ammonites characteristic of the $Q$. mariae zone could be related to the fact that in the Bearpaw Mountains the Jurassic shales near the domes have been faulted, intruded by dikes and sills, and slightly metamorphosed.

Similarly, in the Little Rocky Mountains, where the lower shale member of the Swift Formation ranges from 27 to $32 \mathrm{~m}$ in thickness, Lamberticeras occurs in place in the lower 18 to $20 \mathrm{~m}$, and ammonites characteristic of the Cardioceras cordatum zone are found in the upper 3 to $6 \mathrm{~m}$. Evidently, the Quenstedoceras mariae zone could be represented by 3 to $6 \mathrm{~m}$ of beds from which no ammonites were obtained.

In Montana, ammonite genera and subgenera characteristic of the Cardioceras cordatum zone of western Europe have been found at several localities. For example, in the Little Rocky Mountains $1.6 \mathrm{~km}$ southwest of Landusky at USGS Mesozoic loc. 19205, the upper $6 \mathrm{~m}$ of the lower shale member of the Swift Formation has furnished C. (Scarburgiceras) schucherti Reeside and $C$. (Cawtoniceras) whiteavesi Reeside. In the same mountains on the west side of Zortman Butte at USGS 
Mesozoic loc. 30102, the upper $6 \mathrm{~m}$ of the lower shale member has furnished $C$. (Scarburgiceras) americanum Reeside, C. (S.) cf. C. (S.) crookense Reeside, C. (S.) cf. C. (S.) wyomingense Reeside, C. (Cawtoniceras) whiteavesi Reeside, and C. (Subvertebriceras) cf. C. (S.) canadense Whiteaves.

In the Big Snowy Mountains southwest of Piper, the basal $6 \mathrm{~m}$ of the lower shale member from bottom to top has furnished $C$. (Scarburgiceras) wyomingense Reeside, Goliathiceras (Goliathites) tumidum (Reeside), and Cardioceras hyatti Reeside (fig. 2). In the Pryor Mountains on the west and south sides of Red dome, the lower $14 \mathrm{~m}$ of the Swift Formation has furnished a succession of ammonites that probably correlate with the middle of the European Cardioceras cordatum zone on the basis of the association of the subgenus Scarburgiceras, Goliathites, Subvertebriceras, Maltoniceras, and Cardioceras. However, if an incorrect subgeneric assignment has been made for the new species herein described as $C$. (Scarburgiceras) bighornense Imlay, then the ammonites collected from $6.8 \mathrm{~m}$ above the base of the Swift Formation at Red dome could represent the upper part of the Cardioceras cordatum zone or even the lower part of the Perisphinctes plicatilis zone.

Marine fossils definitely of middle Oxfordian Age have been found at only one place in Montana. The evidence consists of one ammonite (pl. 22, fig. 4) resembling Cardioceras (Scoticardioceras) whitfieldi Reeside (1919, p. 34, pl. 6, figs. 1) that was found with many specimens of Buchia concentrica (Sowerby) in $4.5 \mathrm{~m}$ of grayishblack fissile shale exposed at the south edge of Glacier National Park along the Burlington Railway about 4.8 $\mathrm{km}$ southwest of the village of Summit, Mont. (fig. 1). This occurrence is of interest stratigraphically because it is at the top of the lower shale member of the Swift Formation just beneath $17 \mathrm{~m}$ of thin- to thick-bedded to massive sandstone that underlies the Morrison Formation. It is likewise of interest chronologically because it suggests (1) that Buchia concentrica does occur as low as the Perisphinctes plicatilis zone; (2) that an occurrence of Buchia concentrica in the basal bed of the Swift Formation on Diversion Ridge a little south of the Sun River in the $\mathrm{NW}^{1 / 4} \mathrm{SW}^{1 / 4}$ sec. 12, T. 21 N., R. 9 W., (Mudge, 1972, p. A48) does not imply that the basal bed is younger than the Perisphinctes plicatilis zone; (3) that Buchia concentrica may be associated with Cardioceras in the Bowser Basin of British Columbia (Frebold and Tipper, 1975, p. 157); and (4) that the upper sandstone member of the Swift Formation throughout at least northern Montana is probably of middle to late Oxfordian Age, younger than the P. plicatilis zone.

\section{REDWATER MEMBER OF THE STUMP FORMATION}

The Redwater Member of the Stump Formation (fig. 9) correlates basally with the Cardioceras cordatum zone of western Europe, as shown by the association of the subgenera Pachycardioceras, Cardioceras, Scarburgiceras, and Vertebriceras (fig. 3) only $5 \mathrm{~m}$ above the base of the member near Dinosaur Quarry in northeastern Utah (USGS Mesozoic loc. 11256). Such a correlation is confirmed by the association of the subgenera Cardioceras and Scarburgiceras $4.5 \mathrm{~m}$ above the base of the member at Lily Park in the northwest corner of Colorado (USGS Mesozoic loc. 27730). The higher beds could correlate with the $C$. cordatum zone or with the lower part of the $P$. plicatilis zone, as shown by the presence of (1) C. (Cardioceras) by itself near the base of the Redwater Member of the Stump at McCoy Creek, Idaho; and (2) C. (Sagitticeras) in the upper $9 \mathrm{~m}$ of the member on Little Brush Creek in northeast Utah (fig. 3). Evidently the upper part of the Redwater Member of the Stump correlates at least partly with the Cardioceras cordatum zone and perhaps partly with the lower part of the Perisphinctes plicatilis zone. Inasmuch as the Redwater Member at Dinosaur Quarry is overlain unconformably by the Windy Hill Sandstone Member of the Sundance Formation, as in central, south-central, and eastern Wyoming, it is herein correlated with the $C$. cordatum zone as in those areas.

\section{REDWATER SHALE MEMBER OF THE SUNDANCE FORMATION}

In the Bighorn Basin (fig. 8), the lower part of the Redwater Shale Member of the Sundance Formation is correlated with the Cardioceras cordatum zone on the basis of the association of the ammonite subgenera Cardioceras, Vertebriceras, Cawtoniceras, and Scarburgiceras (fig. 7). The upper part may also be of that age on the basis of an occurrence of Scarburgiceras reported to be only $6 \mathrm{~m}$ below the top of the member on the east side of Little Sheep Mountain (USGS Mesozoic loc. 26717). This occurrence, however, needs checking in the field because (1) the ammonite Scarburgiceras bighornense was obtained from a highly folded area according to the collector; (2) preservation of the specimen indicates that it occurred in a concretionary bed typical of the lower shale unit of the member but not typical of the upper sandstone unit; (3) the ammonite has been collected elsewhere $14 \mathrm{~m}$ below the top of the lower shale unit of the Redwater Shale Member on the west side of Little Sheep Mountain (fig. 4); and (4) the ammonite also has been collected $2.4 \mathrm{~m}$ below the top of the lower shale member of the Swift Formation at Red dome on the west side of the Pryor Mountains in south-central Montana (fig. 2).

In the Wind River Basin (fig. 9), the entire Redwater Shale Member correlates with the Cardioceras cordatum zone on the basis of the presence of $C$. (Subvertebricera) canadense (Whiteaves) 9-15 $\mathrm{m}$ above the base of the member about $8 \mathrm{~km}$ west-northwest of Lander (USGS Mesozoic loc. 30104) and Cardioceras distans (Whitfield) $6 \mathrm{~m}$ below the top of the member on the Lander anticline (USGS Mesozoic loc. 21090). 
This dating is confirmed by some of the most fossiliferous sequences of the Redwater Shale Member exposed in southeastern Wyoming (fig. 5). For example, the lower part of the member is dated as not older than the Cardioceras cordatum zone by the presence of the subgenera Cardioceras and Vertebriceras about $10.6 \mathrm{~m}$ above the base of the member in the southwest Freezeout Mountains and by the subgenus Cardioceras about 4.5-6 $\mathrm{m}$ above the base of the member in the west Flat Top anticline. Similarly, an early Oxfordian Age not younger than the $C$. cordatum zone is shown by the association of the subgenera Scarburgiceras and Cardioceras only $6 \mathrm{~m}$ below the top of the member in the southwest Freezeout Mountains.

This dating is confirmed by the studies of Sykes and Callomon (1979, p. 847), who have shown that on specimens of Cardioceras from the $C$. cordatum zone, the secondary ribs trend strongly forward from the ventrolateral shoulders to a finely serrated keel. In contrast, on specimens of Cardioceras from the overlying zone, the secondary ribs on the ventrolateral shoulders are separated by a smooth, or nearly smooth, area from a coarsely serrated keel. This dating is suggested also by the fact that the Boreal Cardioceratidae in Eurasia spread farthest south during early 0xfordian time (Sykes and Callomon, 1979, p. 844).

In the Black Hills (fig. 9) of northeastern Wyoming and western South Dakota, the same dating is confirmed by the ammonites present in the lower and middle parts of the Redwater Shale Member (fig. 6). Thus, the subgenera Vertebriceras and Cardioceras southeast of Minnekakta, S. Dak., are found $1 \mathrm{~m}$ and also $10.6 \mathrm{~m}$ above the base of the member and are associated with Pavloviceras at their highest occurrence. Scarburgiceras is found at many places at positions ranging from $4 \mathrm{~m}$ above the base to within $12 \mathrm{~m}$ of the top of the member. Goliathites occurs at positions ranging from 11.5 to $16 \mathrm{~m}$ above the base. The subgenus Goliathiceras on the west side of Beaver Creek (Imlay, 1947, p. 271) occurs $18 \mathrm{~m}$ below the top of the member, which at that place is about $46 \mathrm{~m}$ thick. These subgenera, judging by their stratigraphic ranges in Europe (fig. 7) and by the association of Pavloviceras with Cardioceras, show that the ammonite-bearing beds are not younger than the $C$. cordatum zone, although most of the associated genera and subgenera range higher. Nonetheless, in the Black Hills, the upper 12 to $18 \mathrm{~m}$ of the member, which has not yielded any ammonites, could be slightly younger.

\section{PALEOGEOGRAPHIC AND FAUNAL SETTING}

The ammonites of latest Callovian and Oxfordian Age in the Western Interior region lived in a shallow to fairly shallow sea that entered the Williston Basin from the northwest through southwestern Alberta and British Columbia (Frebold and others, 1959, p. 12-19) and extended as far south as the present area of northern Colorado (Imlay, 1980, figs. 8, 9). The sea derived most of its sediment from the west but received some from other directions. In latest Callovian time, this sea extended only as far as north-central and northeastern Montana and adjoining parts of North Dakota and Saskatchewan, but in late early Oxfordian time, it spread rapidly southward across areas that included most of Wyoming and parts of adjoining States. During early middle Oxfordian time, corresponding to the Perisphinctes plicatilis zone of western Europe, this sea withdrew as far north as the south side of the Wind River Basin in central Wyoming and the Black Hills in northeastern Wyoming and western South Dakota. During that withdrawal, considerable erosion took place in areas from which the sea withdrew.

The sea advanced southward again at least as far as it had earlier in the Oxfordian. The transgression was shorter lived and shallower, however, as shown by (1) its sediments being much thinner, (2) consisting mostly of ripple-marked sandstone, and (3) containing only a few marine molluskan genera that include oysters and belemnites but no ammonites. After deposition of an average thickness of $4.5 \mathrm{~m}$ of sediment, the sea retreated northward into Montana but apparently remained in the northern and northeastern parts of that State during at least part of late Oxfordian time, as shown by the presence of the bivalve Buchia concentrica (Sowerby) 17 to $21.5 \mathrm{~m}$ below the top of the Swift Formation at the southern edge of Glacier National Park as well as at the base of that formation about $96 \mathrm{~km}$ to the southsoutheast near Sun River Canyon. Apparently, marine waters persisted in northern Montana from latest Callovian until late Oxfordian time and did not extend as far west as the Sawtooth Range south of Glacier Park until middle Oxfordian time.

The ammonites that lived in this sea during Callovian and Oxfordian time are mostly of boreal origin as they belong almost entirely to the Family Cardioceratidae and closely resemble, or are in part identical with species that lived farther northwest in western Canada (Frebold, 1964, p. 47-49; Frebold and others, 1959; Frebold and Tipper, 1975) and in Alaska (Reeside, 1919, p. $9,18,21,25,27,28,31,33)$. The ammonites differ mainly by not being associated with Phylloceras as were those in the Bowser Basin of British Columbia and in southern Alaska (fig. 10). The only ammonites in the Western Interior region that are distinctly of Tethyan origin are Poculisphinctes and Prososphinctes.

\section{GEOGRAPHIC DISTRIBUTION}

The geographic occurrences of the ammonites described herein are shown on figure 1 and tables 2 to 6 and are described in detail in table 7. 
TABLE 2.-Geographic distribution of latest Callovian and Oxfordian ammonites in the Swift Formation in Montana [All one- and two-digit numbers refer to localities on fig. 1. Vertical numbers are USGS Mesozoic locality numbers]

\begin{tabular}{|c|c|c|c|c|c|c|c|c|c|c|c|c|c|c|c|c|c|c|c|c|c|c|c|c|c|c|c|c|}
\hline \multirow{4}{*}{ Species } & \multicolumn{28}{|c|}{ Swift Formation } \\
\hline & Autumn & \multicolumn{16}{|c|}{ Bearpaw Mountains } & \multicolumn{11}{|c|}{ Little Rocky Mountains } \\
\hline & 1 & 2 & 3 & \multicolumn{5}{|c|}{4} & \multicolumn{9}{|c|}{5} & \multicolumn{7}{|c|}{6} & 7 & 8 & \multicolumn{2}{|c|}{9} \\
\hline & 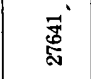 & 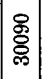 & क्षे & 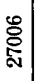 & స్్ㅊ & 离 & 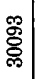 & 兽 & $\begin{array}{l}\overrightarrow{0} \\
\stackrel{5}{\infty} \\
\stackrel{\sim}{*}\end{array}$ & $\begin{array}{l}\stackrel{5}{\sigma} \\
\bar{\sigma}\end{array}$ & $\frac{8}{2}$ & : & 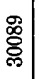 & 若 & 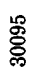 & 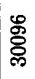 & 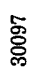 & 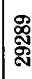 & & 参 & 監 & 胥 & 엻 & $\begin{array}{l}\infty \\
\vdots \\
\stackrel{2}{\circ} \\
\stackrel{2}{\circ}\end{array}$ & $\begin{array}{l}\stackrel{\not}{0} \\
\text { 号 } \\
\end{array}$ & 苟 & 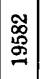 & 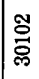 \\
\hline $\begin{array}{l}\text { Quenstedtoceras (Lamberticeras) collieri Reeside } \\
\text { (Pavloviceras) cf. } Q \text { (P.) omphaloides } \\
\text { (J. Sowerby) }\end{array}$ & 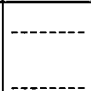 & $\times$ & $x$ & $\times$ & $\times$ & $\times$ & $\times$ & $\times$ & $\times$ & --- & $\times$ & $\times$ & $\times$ & $x$ & $x$ & $x$ & $x$ & $x$ & & $x$ & $x$ & $\ldots$ & $x$ & $x$ & $x$ & $x$ & $x$ & $\ldots$ \\
\hline (P.) cf. Q. (P.) latum (Reeside) & & - & -.-- & - & --. & --- & 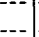 & 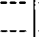 & 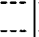 & -... & -... & -.- & --- & $\cdots$ & -- & - & $\cdots$ & & & & - & --- & $-{ }_{--}^{--}$ & - & -- & $-\cdots$ & 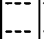 & \\
\hline $\begin{array}{l}\text { Goliathiceras (Goliathites) tumidum (Reeside) } \\
\text { (Pachycardioceras) cf. } Q .(P .) \\
\text { bellefourchense (Reeside) }\end{array}$ & $-\cdots$ & - & $\cdots$ & - & - & --- & - & - & $\cdots$ & - & $\cdots$ & - & - & -- & $-\cdots$ & -- & $\cdots$ & $\cdots$ & & & & & & -3 & - & - & -- & \\
\hline Cardioceras (Cardioceras) hyatti Reeside & & - & -..- & $\ldots$ & -.- & -.- & --- & --. & - & -..- & -..- & .... & -..- & --- & [-- & --. & $\cdots$ & $\ldots$ & & & -..- & -.- & --- & - & - & $\cdots$ & - & \\
\hline (Scarburgiceras) americanum Reeside & & --- & --- & -- & -- & $\cdots$ & $\cdots$ & $\cdots$ & --- & --- & --- & $\cdots$ & $\cdots$ & $\cdots$ & - & $\cdots$ & -- & - & & - & - & - & -- & -- & -- & -- & -- & $\bar{x}$ \\
\hline $\begin{array}{l}\text { (S.) bighornense Imlay, n. sp. } \\
(S .) \text { cf. }(S .) \text { crookense Reeside. }\end{array}$ & & --- & -- & - & --- & - & $\ldots$ & $\cdots$ & $\ldots$ & -- & $--\cdot$ & -- & -- & --- & -- & [-. & -- & - & & $\ldots$ & --- & -- & $--\cdot \mid$ & $\mid--$ & -- & $--\cdot$ & 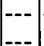 & $\bar{x}$ \\
\hline (S.) schucherti Reeside & & {$\left[\begin{array}{l}--- \\
---\end{array}\right.$} & $\begin{array}{l}--- \\
---\end{array}$ & --- & --- & 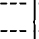 & - & - & --- & --- & --. & --- & - & --- & $\cdots$ & $\ldots$ & $\cdots$ & - & & & -.- & $-\cdots$ & - & - & $-\cdots$ & $\bar{x}$ & $\cdots$ & \\
\hline (S.) wyomingense Reeside & & - & $\begin{array}{l}--- \\
---\end{array}$ & 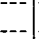 & -- & 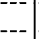 & --- & --. & --- & & -.. & --. & - & 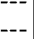 & & & & & & & & & - & - & - & - & - & \\
\hline (S.) cf. $C$. (S.) wyomingense Reeside & & - & \begin{tabular}{l|l}
$\cdots--$ \\
$\cdots-1$
\end{tabular} & - (-- & --- & --- & --. & 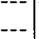 & 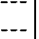 & -.. & 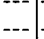 & - & --- & - & -- & $\cdots$ & $\cdots$ & - & & & - & $\ddot{x}$ & --- & - & - & - & $\begin{array}{ll}--- \\
---\end{array}$ & $\bar{x}$ \\
\hline (Scoticardioceras) $\mathrm{cf}$. C. (S.) stillwelli Reeside & & $\cdots$ & \begin{tabular}{l|l}
$-\cdots-$ \\
$-\cdots$
\end{tabular} & $\cdots$ & $\ldots$ &.-- & -.- &.- & -.- & --. & 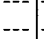 & - & .... & -.- & -- & $\cdots$ & $\cdots$ & - & & & -..- &.-- & --. & - & - & - & - & \\
\hline (S. ) cf. C. (S. ) whitfieldi Reeside & x & - & 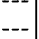 & $\ldots$ & $\ldots$ & 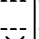 & --. & - & --- & - & 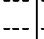 & --. & ... & -..- & $\ldots$ & - & $\cdots$ & & & & $\ldots$ & $\ldots$ & - & - & - & - & - & \\
\hline (Cawtoniceras) whiteavesi Reeside & & - & $\ldots$ & $\ldots$ & - & $x$ & & -- & - & $\times$ &.-- & $\ldots$ & $\ldots$ & -.- & $\cdots$ & -- & $\cdots$ & & & & .. & $\ldots$ & $\ldots$ & $\ldots$ & $\ldots$ & $x$ & -- & $x$ \\
\hline (Maltoniceras) sundancense Reeside & & - & --- & - & $\ldots$ & $x$ & - & - & - & -- & 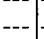 & - & $\ldots$ & $\cdots$ & - & ... & - & & & & - & - & - & - & - & -- & -- & \\
\hline (M. ?) reddomense Imlay, n. sp. & & $\ldots$ & --- & $\ldots$ & $\ldots$ & $\ldots$ & --- & --- & -- & -- & - & -- & $\ldots$ & $\ldots$ & ..- & $\cdots$ & $\cdots$ & & & & -- & -- & -- & -- & --- & & --- & \\
\hline (Subvertebriceras) canadense Whiteaves & & & & $\ldots$ & $\ldots$ & $\ldots$ & & & - & $x$ & - & - & - & $\ldots$ & - & 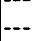 & . & & & & - & -- & - & - & - & & $-\cdots$ & $x$ \\
\hline (S.) cf. $C$. (S.) canadense Whiteaves ... & & & - & - & - & - & -- &.-- & -- & $\times$ & -- & - & -- & -- & - & - & $\ldots$ & & &. & $\cdots$ & $\ldots$ & -- & -- & --- & --- & --- & $x$ \\
\hline (Vertebriceras) stantoni Reeside & & $-\cdots$ & & - & - & $\times$ & & $\ldots$ & -- & -- & -- & - & -- & & - & $\cdots$ & & & & & $\ldots$ & $-\ldots$ & $\ldots$ & -- & --- & & $-\cdots$ & \\
\hline (V.) cf. C. (V.) haresi Reeside & & & $\cdots$ & $\cdots$ & $\ldots$ & $\ldots$ & $\ldots$ & $\ldots$ & -- & & --- & --- & -- & --- & - & $\cdots$ & $\cdots$ & & & $\cdots$ & -- & $-\cdots$ & --- & --- & & $-\cdots$ & --- & \\
\hline Grossouvria? sp. & & -- & --- & $\cdots$ & - & -- & -- & -- & -- & - & $\cdots$ & $\cdots$ & -- & --- & - & - & - & & & & -- & -- & -- & -- & $\ldots$ & -- & -- & \\
\hline Prososphinctes? sp. & & & & & --- & & & & & & & & & & & & & & & & & & -- & -- & & & & \\
\hline
\end{tabular}

TABLE 3.-Geographic distribution of Oxfordian ammonites in the Redwater Member of the Stump Formation in southeastern Idaho and adjoining areas

[All one- and two-digit numbers refer to localities on fig. 1. Vertical numbers are USGS Mesozoic locality numbers]

\begin{tabular}{|c|c|c|c|c|c|c|c|c|c|c|}
\hline \multirow{5}{*}{ Species } & \multicolumn{10}{|c|}{ Redwater Member of Stump Formation } \\
\hline & \multirow{3}{*}{$\begin{array}{c}\text { Idaho } \\
\begin{array}{c}\text { McCoy } \\
\text { Creek }\end{array} \\
19\end{array}$} & \multirow{3}{*}{$\begin{array}{c}\text { Wyoming } \\
\text { Fort } \\
\text { Hill } \\
20\end{array}$} & \multicolumn{6}{|c|}{ Utah } & \multicolumn{2}{|c|}{ Colorado } \\
\hline & & & \multicolumn{6}{|c|}{ Uinta Mountains } & \multirow{2}{*}{\begin{tabular}{|c|} 
Little \\
Snake \\
River \\
26 \\
\end{tabular}} & \multirow{2}{*}{\begin{tabular}{|c|}
$\begin{array}{c}\text { Lily } \\
\text { Park }\end{array}$ \\
27 \\
\end{tabular}} \\
\hline & & & 21 & 22 & 23 & 24 & \multicolumn{2}{|c|}{25} & & \\
\hline & 吕 & 璦 & :̊心 & క్ర్టి & ஜू్ & 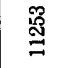 & $\stackrel{\mathscr{3}}{\rightrightarrows}$ & $\underset{\infty}{\stackrel{\infty}{*}}$ & $\overline{\text { के }}$ & 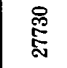 \\
\hline \multirow{2}{*}{\multicolumn{11}{|c|}{$\begin{array}{l}\text { Quenstedtoceras (Goliathites) cf. Q. (G.) hoveyi (Reeside) } \\
\text { Goliathiceras (Pachycardioceras) russelli (Reeside) }\end{array}$}} \\
\hline & 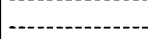 & -4 & ..... &.- & & $\ldots$ & $x$ & & |---o- & \\
\hline \multirow{6}{*}{$\begin{array}{l}\text { Cardioceras (Cardioceras) hyatti Reeside } \\
\text { (C.) sp. } \\
\text { (Scarburgiceras) cordiforme (Meek and Hayden) } \\
\text { (S.) cf. C. (S.) schucherti Reeside } \\
\quad(S .) \text { wyomingense Reeside } \\
\quad(S .) \text { sp. } \\
\text { (Vertebriceras) stantoni Reeside } \\
\text { (Sagitticeras) cf. C. (S.) obtusum Reeside }\end{array}$} & $x$ & $x$ & & & & $-x$ & $x$ & $x$ & $-\cdots$ & $x$ \\
\hline & & & & & & & $x$ & & & \\
\hline & & & & & & & & & $x$ & - \\
\hline & & $x$ & & & & & $x$ & $-\cdots$ & & \\
\hline & $x$ & |--.--.-- & -..... & & & & $x$ & & & $x$ \\
\hline & & & ------ & $x$ & $x$ & & & & & \\
\hline
\end{tabular}


TABLE 2.-Geographic distribution of latest Callovian and Oxfordian ammonites in the Swift Formation in Montana-Continued [All one- and two-digit numbers refer to localities on fig. 1. Vertical numbers are USGS Mesozoic locality numbers]

\begin{tabular}{|c|c|c|c|c|c|c|c|c|c|c|c|c|c|c|c|c|c|c|c|c|c|c|c|c|c|c|c|}
\hline \multirow{4}{*}{ Species } & \multicolumn{27}{|c|}{ Swift Formation } \\
\hline & \multicolumn{12}{|c|}{ Little Rocky Mountains-Continued } & \multicolumn{6}{|c|}{$\begin{array}{l}\text { Big Snowy } \\
\text { Mountains }\end{array}$} & \multicolumn{9}{|c|}{ Pryor Mountains } \\
\hline & \multicolumn{4}{|c|}{10} & \multicolumn{5}{|c|}{11} & \multicolumn{3}{|c|}{12} & 13 & \multicolumn{4}{|c|}{14} & 15 & 16 & \multicolumn{5}{|c|}{17} & \multicolumn{3}{|c|}{18} \\
\hline & 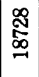 & 芯 & 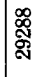 & ळ్ & 疍 & 象 & 疋 & 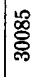 & 怘 & 哭 & 姿 & 咢 & 들 & $\stackrel{?}{9}$ & $\stackrel{9}{9}$ & 芶 & జิ & $\overline{\bar{g}}$ & 胥 & 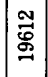 & స్్ㅊ & 蒙 & 总 & 옳 & 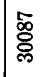 & 宊 & ठั. \\
\hline $\begin{array}{l}\text { Quenstedtoceras (Lamberticeras) collieri Reeside } \\
\text { (Pavloviceras) cf. } Q \text {. (P.) omphaloides } \\
\text { (J. Sowerby) }\end{array}$ & $x$ & $\begin{array}{l}x \\
x\end{array}$ & $x$ & $x$ & $x$ & $x$ & $x$ & -- & $x$ & $x$ & $x$ & -- & & & -- & -- & --- & -- & - & -- & -- & $-\cdots$ & & & -- & -- & \\
\hline$(P$.$) cf. Q .(P$.$) latum (Reeside)$ & $\cdots$ & $\hat{x}$ & $\mid--$ & & & - & -- & - & & & 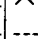 & -- & & & & $\bar{x}$ & - & - & ... & $-\cdots$ & - & $\mid$ & $x$ & & - & 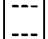 & - \\
\hline $\begin{array}{c}\text { Goliathiceras (Goliathites) tumidum (Reeside) } \\
\text { (Pachycardioceras) cf. } Q .(P .) \\
\text { bellefourchense (Reeside) }\end{array}$ & $-\cdots$ & -- & - & & & -- & - & - & & & $\cdots$ & -- & - & $x$ & - & - & - & $\bar{x}$ & & +- & $-\cdots$ & -- & $x$ & $x$ & - & -- & - \\
\hline Cardioceras (Cardioceras) hyatti Reeside & -- & 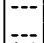 & $-\cdots$ & & & $\cdots$ & $-\ldots$ & & & & & & & $\mathrm{x}$ & - & $\cdots$ & $-\cdots$ & $x$ & & - & - &.- & $\mathrm{X}$ & 1 & - & -- & \\
\hline (Scarburgiceras) americanum Reeside & $\cdots$ & $\bar{x}$ & - & & & $\cdots$ & - & $x$ & & & - & $\mathrm{X}$ & & & & $-\cdots$ & - & - & --..- & $\mid$ & $\bar{x}$ & -- & & $t-$ & $-\cdots$ & 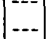 & $x$ \\
\hline (S.) bighornense Imlay, n. sp. & - & -- & -- & & &.-- & -- & --. & & &.- & -.. & & & - & $\ldots$ & -- & - & & --- & $\ldots$ & -- & & $x$ & - &.-- & \\
\hline (S.) cf. (S.) crookense Reeside & -- & - & - & & & -- & - & - & & & $\begin{array}{ll}--1 \\
-\cdots\end{array}$ & 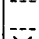 & & & $1-$ & - & - & - & & - & -- & - & & & - & 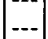 & - \\
\hline (S.) schucherti Reeside & -- & - & - & & & -- & -- & & & & - & $x$ & & & & - & - & - & & - & - & - & & & - & - & \\
\hline (S.) wyomingense Reeside & - & - & - & & & - & - & - & & & - & - & $x$ & & $x$ & - & - & - &.- & -- & $\mid$ & --- & & & - & -- & \\
\hline (S.) cf. $C$. (S.) wyomingense Reeside & & - & - & & & -- & - & & & & - & - & & & - & $\ldots$ & $x$ & - & & -- & $-\ldots$ & -- & & & - & $\mathrm{X}$ & $x$ \\
\hline (Scoticardioceras) cf. C. (S.) stillwelli Reeside & - & - & - & & $x$ & $\cdots$ & -- & - & & & $\cdots$ & --- & & & - & - &.- & - & & $\ldots$ & -1 & - & & & $\ldots$ & -- & \\
\hline (S.) cf. $C$. (S.) whitfieldi Reeside & - & -- & - & & & -- & - & & & & - & - & & & - & - & - & - & & & - & - & & & - & $-\cdots$ & \\
\hline (Cawtoniceras) whiteavesi Reeside & $-\ldots$ & -- & - & & & - & $\ldots$ & --. & & & & ... & & & - & $\ldots$ & -..- & $\ldots$ & $x$ & -- & -- & -- & & & & - & \\
\hline (Maltoniceras) sundancense Reeside & - & $\ldots$ & -- & & & - & --- & - & & & $x$ & - & & & - & - & -- & - & & $x$ & -- & -- & & & $\mathrm{x}$ & $\ldots$ & -- \\
\hline (M. ?) reddomense Imlay, n. sp. & -- & - & $\ldots$ & & & $\ldots$ & -- & $\ldots$ & & & $\ldots$ & -- & & & - & - & $\ldots$ & -- & & $x$ & -- & & $\mathrm{x}$ & & $x$ & --- & -- \\
\hline (Subvertebriceras) canadense Whiteaves & $\ldots$ & -- & $-\cdots$ & & & $\ldots$ & -- & - & & & $\cdots$ & - & & & - & - & $\cdots$ & -- & & $x$ & $x$ & $x$ & $x$ & & $x$ & -- & \\
\hline$(S$.$) cf. C .(S$.$) canadense Whiteaves$ & -- & -- & -- & & & --- & -- & -- & & & -- & -- & & & - & $-\cdots$ & -- & $\ldots$ & & --- & $\cdots$ & -- & & & -- & $-\cdots$ & -- \\
\hline (Vertebriceras) stantoni Reeside & -- & --- & $-\cdots$ & & & -- & -- & -- & & & $-\cdots$ & - & & & - & $-\cdots$ & -- & -- & & -- & -- & --- & $-\because$ & $x$ & -- & -- & -- \\
\hline (V.) cf. C. (V.) haresi Reeside & $\ldots$ & -- & -- & & &.- & -- & - & & & & & & & & $\ldots$ & 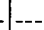 & -- & & --- & -- & $\ldots$ & $X$ & & & --- & \\
\hline Grossouvria? sp. & -- & $--:$ & - & & & -- & -- & - & & & $x$ &.- & & & - & $-\ldots$ & -- & - & -- & $\ldots$ & $-\cdots$ & -- & $-\cdots$ & & $-\cdots$ & $-\cdots$ & $-\cdots$ \\
\hline Prososphinctes? sp. & & & & & $x$ & -- & $-\cdots$ & -- & & & - & & & & -. & $\ldots$ & -- & -- & & --- & $\ldots$ & & & & --- & $-\cdots$ & - \\
\hline
\end{tabular}

TABLE 4.-Geographic distribution of Oxfordian ammonites in the Redwater Shale Member of the Sundance Formation in the Bighorn and Wind River Basins of Wyoming

[All one- and two-digit numbers refer to localities on fig. 1. Vertical numbers are USGS Mesozoic locality numbers.]

\begin{tabular}{|c|c|c|c|c|c|c|c|c|c|c|c|c|c|c|c|c|c|c|c|}
\hline \multirow{4}{*}{ Species } & \multicolumn{19}{|c|}{ Redwater Shale Member of Sundance Formation } \\
\hline & \multicolumn{16}{|c|}{ Bighorn Basin } & \multicolumn{3}{|c|}{$\begin{array}{l}\text { Wind } \\
\text { River } \\
\text { Basin }\end{array}$} \\
\hline & \multicolumn{6}{|c|}{28} & \multicolumn{4}{|c|}{29} & 30 & \multicolumn{4}{|c|}{31} & \multirow{2}{*}{$\frac{32}{\text { ஜ }}$} & \multirow{2}{*}{$\frac{33}{\underset{0}{0}}$} & \multirow{2}{*}{$\frac{34}{\text { §్ }}$} & \multirow{2}{*}{$\frac{35}{\stackrel{్}{乛 ్}}$} \\
\hline & 동 & 胥 & 点 & $\underset{⿱ 乛}{\mathbb{N}}$ & 号 & 营 & $\frac{\overrightarrow{5}}{\frac{m}{n}}$ & 药 & 蛋 & 营 & 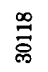 & 愛 & 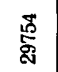 & ?̊ㅠㅇ & 莡 & & & & \\
\hline \multirow{7}{*}{ 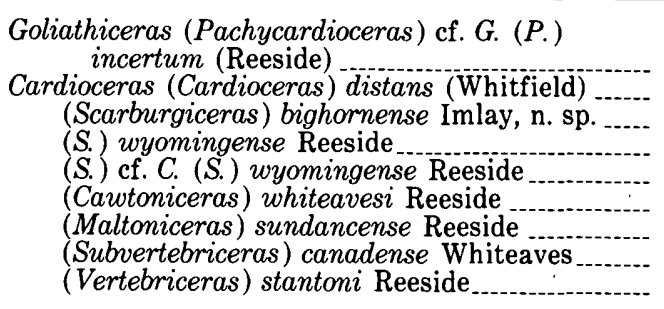 } & & 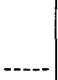 & & & & & & & & & & & & & $x$ & & & & \\
\hline & $\mathrm{x}$ & $x$ & & $x$ & & & 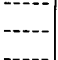 & $x$ & & & $x$ & & & $x$ & & & 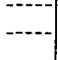 & $x$ & \\
\hline & -..- & & & & $x$ & $x$ & $\cdots$ & 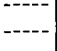 & 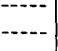 & & 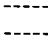 & $x$ & $\cdots$ & 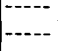 & & $x$ & & & $-\cdots$ \\
\hline & $\cdots$ & $\cdots$ & & & & & $\cdots$ & $-\cdots$ & 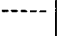 & & & & $\bar{x}$ & 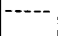 & & & & & $-\bar{x}$ \\
\hline & $\cdots$ & 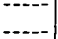 & -.. & - & & $x$ & $\cdots$ & - n & & & & $\cdots$ & & & & & & $\cdots$ & 入 \\
\hline & $\cdots$ & & & & & & $-\cdots$ & -....... & 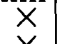 & & & & & & & & $x$ & 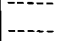 & 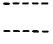 \\
\hline & & & & & & & & & & & & $-\ldots$ & & & & $x$ & $-\cdots$ & & \\
\hline
\end{tabular}


TABLE 5.-Geographic distribution of Oxfordian ammonites in the Redwater Shale Member of the Sundance Formation in central, south-central, east-central, and southeastern Wyoming

[All one- and two-digit numbers refer to localities on fig. 1. Vertical numbers are USGS Mesozoic locality numbers.]

\begin{tabular}{|c|c|c|c|c|c|c|c|c|c|c|c|c|c|c|c|c|c|c|c|c|c|c|c|c|c|c|c|c|c|c|c|c|c|c|c|c|c|c|c|c|c|}
\hline \multirow{5}{*}{ Species } & \multicolumn{41}{|c|}{ Redwater Shale Member of the Sundance Formation } \\
\hline & \multicolumn{41}{|c|}{ Southeastern Wyoming } \\
\hline & \multirow{2}{*}{\multicolumn{2}{|c|}{\begin{tabular}{|c|}
$\begin{array}{l}\text { Carbon } \\
\text { County }\end{array}$ \\
36 \\
\end{tabular}}} & \multirow{2}{*}{\multicolumn{3}{|c|}{\begin{tabular}{|c|c}
$\mathrm{n}$ & $\begin{array}{c}\text { Natrona } \\
\text { County }\end{array}$ \\
37 \\
\end{tabular}}} & \multicolumn{3}{|c|}{$\begin{array}{l}\text { Albany } \\
\text { County }\end{array}$} & \multicolumn{26}{|c|}{ Carbon County } & \multicolumn{5}{|c|}{ Converse County } & \multirow{2}{*}{ 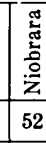 } & \multirow{2}{*}{ 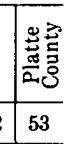 } \\
\hline & & & & & & 38 & & & & 42 & & 43 & & & 44 & & & 45 & & & & & 46 & & & & 47 & & & & $4 \varepsilon$ & & & 49 & & 50 & & 5 & & & \\
\hline & $\stackrel{8}{\Xi}$ & $\mid$ & 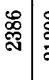 & & 趸 & 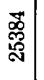 & 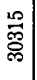 & 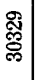 & 要 & 窟 & 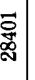 & 总 & 窇 & & 敢 & : & 常 & & 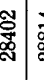 & & 怘 & $\stackrel{\circ}{:}$ & $\begin{array}{l}0 \\
0 \\
0 \\
0\end{array}$ & 芯 & 麜 & 恋 & 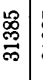 & 点 & 点 & 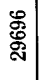 & 吕 & 离 & E & $\begin{array}{l}\mathscr{0} \\
\mathbb{0} \\
\approx \\
a\end{array}$ & 总 & นั้ & 蛋 & & 熵 & 总 & 藏 \\
\hline Quenstedtoceras (Pavloviceras) latum (Reeside & & & & & & & & & & & & & & & & & & & & & & & & & & & & & & & & $x$ & & 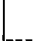 & & 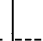 & 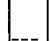 & & & & \\
\hline Goliathiceras (Goliathiceras) suspectum & $\ldots$ & & & & - & & & & & & & 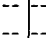 & & & 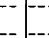 & & & & & & & & & & $x$ & & & & & $x$ & $\ddot{x}$ & & 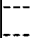 & $\ldots$ & & $\ldots$ & .. & & $\cdots$ & & \\
\hline (Goliathites) hoveyi (Reeside) & & ... & $\bar{x}$ & & & & & $=-$ & & & & & & & & & & & & & & & -.. & & & & & & & & $\hat{x}$ & $\bar{x}$ & . & $\ldots$ & & & & & $\cdots$ & & \\
\hline (Pachycardioceras) albaniense (Reeside) & --- & -.. & & & - & & & $\cdots$ & & & & 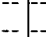 & & & - & - & & & & & & $\bar{x}$ & - & & 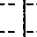 & -- & .. & & - & ...... & $\ldots$ & -... & - & -. & & - & - & $\ldots$ & 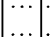 & & $\ldots$ \\
\hline (P. (bellefourchense (Reeside) ............... & $\ldots$ & & & & & & & 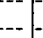 & & & & & & & $\ldots$ & & & & & & & & $\cdots$ & & & -. & $\ldots$ & & & . & -.- & $\bar{x}$ & -- & - & -. & $\ldots$ & $\ldots$ & $\cdots$ & $\left|\begin{array}{c}\cdots \\
\cdots\end{array}\right|$ & & \\
\hline (P.) crassum (Reeside) & -- & .. & & & & -.. & - & ... & & & & .. & & & -.... & - & - & - & $\ldots$ & -- & 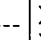 & $\times$ & ... & & - & -- & $\times$ & & & $\ldots$ & -- & --- & --- & -- & & $-\ldots$ & $\ldots$ & -- & ... & & \\
\hline (P.) incertum (Reeside) & - & . & & & & $\ldots$ & - & - & & & $x$ & -- & 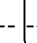 & & - & & 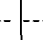 & - & - & & $\cdots$ & $\times$ & $\ldots$ & & 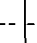 & - & $\cdots$ & & & & - & $-\bar{z}$ & -- & -- & & - & $-\cdots$ & -- & .... & & \\
\hline $\begin{array}{l}(P .) \text { russelli (Reeside) } \\
(P .) \text { cf. } C .(P .) \text { russelli (Reeside) }\end{array}$ & -- & $\cdots$ & & & & - & & 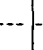 & & & & -- & & & -- & $\bar{x}$ & $x$ & & & & & & $\cdots$ & & & & & & & & & & & -- & & -- & -- & -- & $\cdots$ & & \\
\hline $\begin{array}{l}(P .) \text { cf. C. }(P .) \text { russelli (Reeside) } \\
\text { Cardioceras (Cardioceras) auroraense Reeside }\end{array}$ & & $\cdots-$ & & & & & & & & & & & & & & & & & & & & & & & & & & & & & & $|\bar{x}|$ & & $\cdots$ & & & & $\cdots$ & $\cdots$ & & \\
\hline (C.) distans (Whitfield) & $-\ldots$ & -1 & & & & & & & & & & & & & $\bar{x}$ & & & & & & & $\ldots$ & ... & & .. & $\bar{x}$ & (t) & $x$ & - & - & - & -.. & -- & --- & & $\cdots$ & -- & - & $\cdots$ & & $\cdots$ \\
\hline (C.) hyatti Reeside & --- & & & & $\times$ & -.- & & & & & & 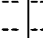 & & & $x$ & $\bar{x}$ & & & & $\bar{x}$ & & -. & $\bar{x}$ & & & & & & & & & & & & & & $\ddot{x}$ & & $\left|\begin{array}{c}\cdots \\
\ldots\end{array}\right|-10$ & & \\
\hline $\begin{array}{l}\text { (Scarburgiceras) cordiforme (Meek and } \\
\text { Hayden) }\end{array}$ & & & & & & & & & & & & & & & & & & & & & & $x$ & & & & & & & & & & & & & & & & & & & \\
\hline (S. ) crookense Reeside & --- & -- & & & & & & & & & & & & & & & & & & & $x$ & & -- & & & & $\cdots$ & & & $\cdots$ & $x$ & -- & -- & - & & - & - & -- & $\cdots$ & & \\
\hline & & & & & & & & & & & & $x$ & -. & & & & 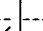 & & & & -- & $x$ & - & & & -- & -- & & & -1 & & & --- & & & & & & & & \\
\hline (S.) wyomingense Reeside & $x$ & $x$ & & $\times$ & & & $\mathrm{x}$ & $x$ & & & & - & & & -. & K & $x$ & & & & -. & & - & $x$ & & & -- & & & $\cdots$ & $\times$ & $\cdots$ & $x$ & $x$ & & 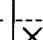 & $x$ & $\times$ & $\mathrm{x}$ & & $\dddot{\mathrm{V}}$ \\
\hline $\begin{array}{l}\text { (S.) cf. C. (S.) wyomingense Reeside } \\
\text { Maltoniceras) plattense Reeside }\end{array}$ & $\ldots$ & - & $\mathrm{x}$ & & & & & $\because$ & & & & -1 & & & & & & & & & & & & & & & & & & -- & & & & & & & & & $\cdots$ & & $x$ \\
\hline (Vertebriceras) stantoni Reeside & & - & & & & & & & & & & & & & $\bar{x}$ & & $\bar{x}$ & $\bar{x}$ & & 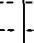 & & & - & & & - & $\bar{x}$ & & & $\ldots$ & $x$ & $\cdots$ & $\bar{x}$ & & & & - & $\cdots$ & $\begin{array}{c}\cdots \\
\cdots\end{array}$ & $\dddot{x}$ & $\ddot{x}$ \\
\hline (V.) haresi Reeside & & & & & & $\times$ & & & & & & & & & & & & & & & & & & & & & & & & 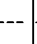 & & & & & & & & & & & \\
\hline
\end{tabular}


TABLE 6.-Geographic distribution of Oxfordian ammonites in the Redwater Shale Member of the Sundance Formation in the Black Hills of northeastern Wyoming and western South Dakota

[All one- and two-digit numbers refer to localities on Fig. 1. Vertical numbers are USGS Mesozoic locality numbers.]

\begin{tabular}{|c|c|c|c|c|c|c|c|c|c|c|c|c|c|c|c|c|c|c|c|c|c|c|c|c|c|c|c|c|c|c|c|c|c|c|c|c|c|c|c|}
\hline \multirow{5}{*}{ Species } & \multicolumn{39}{|c|}{ Redwater Shale Member of Sundance Formation in Black Hills } \\
\hline & \multicolumn{25}{|c|}{ Northeastern Wyoming } & \multicolumn{14}{|c|}{ Western South Dakota } \\
\hline & \multicolumn{21}{|c|}{ Crook County } & \multicolumn{4}{|c|}{$\begin{array}{l}\text { Weston } \\
\text { County }\end{array}$} & \multirow{2}{*}{\multicolumn{3}{|c|}{$\begin{array}{c}\begin{array}{c}\text { Butte } \\
\text { County }\end{array} \\
66 \\
\end{array}$}} & \multirow{3}{*}{ 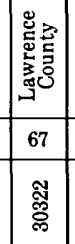 } & \multirow{3}{*}{ 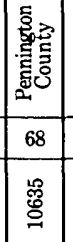 } & \multicolumn{2}{|c|}{$\begin{array}{l}\text { Gunter } \\
\text { County }\end{array}$} & \multicolumn{7}{|c|}{ Fall River County } \\
\hline & \multicolumn{2}{|c|}{54} & \multirow{2}{*}{$\begin{array}{l}55 \\
0 \\
0 \\
0\end{array}$} & & & & 57 & & & & 58 & & & & & 61 & & 62 & & 63 & & 64 & & 65 & & & & & & & 69 & 70 & & 72 & & 73 & & 74 & \\
\hline & & $\begin{array}{l}\text { 惫 } \\
\text { 虽 }\end{array}$ & & $\stackrel{\mathbb{E}}{\mathbb{*}}$ & & 串 & 范 & 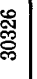 & 胥 & \begin{tabular}{l}
0 \\
$\stackrel{0}{0}$ \\
\hdashline \\
\hdashline
\end{tabular} & 浔 & & & & 方 & 苟 & 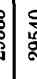 & 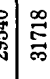 & 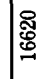 & 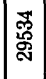 & \begin{tabular}{|l|l|}
0 \\
8 \\
8 \\
8
\end{tabular} & 芯 & 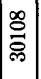 & 总 & 윰 & 总 & 䂛 & 芯 & & & 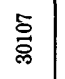 & 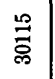 & 总 & & : & 惫 & 랑 & & 变 \\
\hline Quenstedtoceras (Pavloviceras) latum (Reeside) _- & $\ldots$ & & $x \mid$ & $-\ldots$ & & & & $\ldots$ & & $x$ & $\ldots$ & & & & & & & $\ldots$ & .-- & -. & $\mid-$ &.- & $\ldots$ & & -- & -- & $\ldots$ & -- & & ---ol & & & & & -- & & & & \\
\hline (P.) minnekahtense Imlay, n. sp. & $\ldots$ & & & $\ldots$ & $\ldots$ & & & $\ldots$ & -- & $x$ & .. & & -- & & & & & $\ldots$ & $\ldots$ & $\ldots$ & - & -- & -. & - & - & - & -1 & -. & -..- & |- & $\cdots$ & .... & & -- & .- & -. & $\bar{x}$ & - & \\
\hline Goliathiceras (Goliathiceras) suspectum (Reeside) & $\cdots$ & & . & -... & $\ldots$ & & & & & & -- & & $\ldots$ & & & $\bar{x}$ & 人) & & & -- & $\begin{array}{ccc}-- \\
--\end{array}$ & \begin{tabular}{|r}
-- \\
--
\end{tabular} & -- & 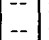 & $\bar{x}$ & - & $\begin{array}{c}-- \\
-\end{array}$ & -- & 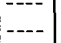 & $x$ & -... & $-\ldots$ & $\ldots$ & -- & -- & -- & .- & -- & $x$ \\
\hline (Goliathites) hoveyi (Reeside) & $\ldots$ & & - & $\times$ & - & -- & & $\ldots$ & - & $x$ & - & & 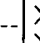 & & & - & - & - & - & -- & -- & -- & -- & - & - & $x$ & -- & -. & $-\ldots$ & --- & $-\cdots$ & -... & -- & -- & -- & .. & -- & - & \\
\hline (G.) tumidum (Reeside) & $\ldots$ & $\bar{x}$ & -... & -.. & $x$ & - & $\ldots$ & -- & -- & $x$ & - & & 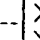 & & - & - & $x$ & $\mid--$ & -- & -- & -- & -- & -- & -- & - & - & -- & -- & $\cdots$ & $-\cdots$ & --- & --- & -- & -- & -- & -- & -- & -- & -- \\
\hline (Reeside) & - & & $-\cdots$ & $\cdots$ & - & & & $\cdots$ & - & - & - & & $-\lambda>$ & & & -- & - & - & & -- & -- & -- & -- & -- & - & - & - & -- & --- & $-\cdots$ & $-\cdots$ & $\cdots$ & -- & -- & $\cdots$ & 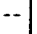 & -- & - & \\
\hline $\begin{array}{l}\text { (Pachycardioceras) bellifourchense (Reeside) } \\
\text { (P.) crassum (Reeside) }\end{array}$ & & & $\cdots-$ & -- & $\cdots$ & & & $\cdots$ & -- & & $\cdots$ & & $--1\rangle$ & & & $\bar{x}$ & & - & & & -- & -- & -- & -- & & - & - & & $-\cdots$ & $x$ & $\cdots$ & $\cdots$ & & -- & - & -- & & $\cdots$ & \\
\hline $\begin{array}{l}(P .) \text { crassum (Reeside) } \\
(P .) \text { incertum (Reeside) }\end{array}$ & $\ldots$ & & - & $\ldots$ & $\ldots$ & ון & & 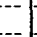 & & -1 & $\cdots$ & & -- & & & & & . & & -- & -- & -- & -- & $\bar{x}$ & -- & -1 & - & -- & --- & A & $\cdots$ & $\cdots$ & -- & & - & - & $\cdots$ & - & \\
\hline (P.) russelli (Reeside) & & $\cdots$ & . & $\ldots$ & . & & & & & $\bar{x}$ & & & & & & & & & & & $\ldots$ & $\cdots$ & $\cdots$ & ... & - & . & $\ldots$ & - & - & $\cdots$ & $\cdots$ & -- & 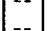 & $\bar{x}$ & & -- & $\cdots$ & & \\
\hline Cardioceras (Cardioceras) auroraense Reeside & $\ldots$ & - & |. & $\cdots$ & $\ldots$ & -- & & ... & -... & & - & & $\ldots$ & & $\ddot{x}$ & 人) & . & $\cdots$ & & $|-|$. &.-- & \begin{tabular}{|c}
-- \\
-
\end{tabular} & {$\left[\begin{array}{cc}- \\
--\end{array}\right.$} & $-\overline{-}$ & $\ldots$ & $\ldots 1$ - & -1 & $-\overline{-}$ & -a. & $\bar{x}$ & - & $\cdots$ & $\mid$\begin{tabular}{l|l}
-- \\
--
\end{tabular} & $\mathrm{x}$ & $\bar{x}$ & -1 & -- & & \\
\hline (C.) distans (Whitfield) & 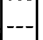 & --- & $x$ & .... & . & & & ... & - & $x$ & $\ldots$ & & $\ddot{x} \ddot{\gamma}$ & & $x$ & < & & $x$ & $\bar{x}$ & - & -- & $\cdots$ & {$[-1$} & - & - & 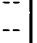 & $\begin{array}{l}-- \\
-1\end{array}$ & -1 & 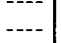 & $-\cdots$ & 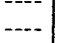 & 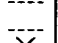 & - &.- & - & $\cdots$ & $\ddot{x}$ & $\bar{x}$ & \\
\hline (C.) hyatti Reeside & $\ldots$ & - & $\ldots$ & $\ldots$ & $\ldots$ & & & $\ldots$ & -. & $\ldots$ & - & $\ldots$ & -. & & & $-\cdots$ & $\ldots$ & $-\ldots$ & 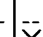 & --- & -- & -- & -- & -- & -- & -- & -- & -- & $---\mid$ & --- & --- & $\times$ & $\mid-$. & .. & -- & -- & --1 & $\ldots$ & \\
\hline (Scarburgiceras) americanum Reeside & - & $\bar{x}$ & $\cdots$ & -- & - & & & 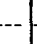 & - & & $\times$ & - & -- & & $x$ & - & & $\ldots$ & $x$ & -- & -- & -- & -- & -- & -- & -- & - & -- & $\cdots$ & -- & ---- & --- & -- & -- &.- & -- & -- & -- & \\
\hline (S.) cordiforme (Meek a & $\cdots$ & -- & - & $\cdots$ & $\cdots$ & & & $\cdots$ & $\cdots$ & $\times 1$ & -- & $\times-$ & $\cdots$ & & & $\cdots$ & - & $-\cdots$ & & -- & -- & -- & -- & -- & - & $\bar{x}$ & -- & -- & $\cdots$ & $---\rangle$ & --- & $-\cdots$ & $-\cdots$ & -- & -- & -- & -- & -- & \\
\hline (S.) crookense Reeside & $\cdots$ & -- & $x$ & $\cdots$ & $\cdots$ & & & $\cdots$ & -- & & $\cdots$ & & -- & & & - & - & $\ldots$ & - & -- & -- & -- & $-\cdot$ & -- & -- & $x$ & -1 & -- & $-\cdots$ & --- & $-\cdots$ & $---\mid$ & -- & $\bar{x}$ & -- & $\bar{x}$ & -- & -- & \\
\hline $\begin{array}{l}\text { S.?) mathiaspeakense Imlay, n. sp. } \\
\text { (S.) reesidei Maire }\end{array}$ & & & $\left.\ddot{x}\right|^{-}$ & $\cdots$ & $\cdots$ & & & -1 & -1 & $\bar{x}$ & 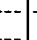 & $\bar{x}$ & -- & & & 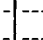 & & $\cdots$ & & -- & -- & -- & {$[-]$} & -- & -- & -- & -1 & $-\cdots$ & ---- & ---- & $\cdots-$ & $\cdots$ & -- & & $\cdots$ & ${ }^{x}$ & -- & & \\
\hline $\begin{array}{l}\text { (S.) reesider Marre } \\
\text { (S.) wyomingense Reeside }\end{array}$ & $x$ & & 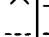 & $\cdots$ & 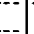 & 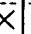 & $x$ & $\bar{x}$ & $\bar{x}$ & & $\bar{x}$ & $x$ & & & & & & -- & $-\bar{x}$ & $\bar{x}$ & $\cdots$ & $\bar{x}$ & $\bar{x}$ & {$[-$} & -- & $\ddot{x}$ & $\ddot{x}$ & -- & $-\bar{x}$ & $\cdots$ & $\cdots$ & $-\bar{x}$ & - & - & -- & - & -- & -- & \\
\hline$(S$.$) cf. C .(S$.$) wyomingense Reeside$ & -.. & $\bar{x}$ & $-\cdots$ & -..- & 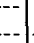 & & & & & & & & & & & & & - & & & - & $\mid \begin{array}{l}3 \\
--\end{array}$ & {$\left[\begin{array}{l}1 \\
--\end{array}\right.$} & $\ddot{x}$ & -- & \begin{tabular}{c|c}
- \\
.-
\end{tabular} & $\begin{array}{l}-. \\
-.\end{array}$ & $\bar{x}$ & … & - & $x$ & a. & $\ddot{x}$ & $\begin{array}{l}-- \\
--\end{array}$ & -1 & -- & $\begin{array}{l}-- \\
-.\end{array}$ & 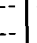 & \\
\hline coticardioceras) stillwelli Reeside & $\ldots$ & & $\ldots$ & ... & $\ldots$ & & & -.. & -. & & 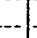 & & & & & & & $\ldots$ & & & -. & -- & .. & -- & -1 & -- & -- & $\ldots$ & $-\ldots$ & ---- & $\ldots$ & $-\cdots$ & -- & -. & -- & -. & -- & & \\
\hline & $\ldots$ & & -...... & $\ldots$ & $\ldots$ & & & $\ldots$ & ... & & & -1 & $x$ & & & & & $\ldots$ & & -- &.- & -- & {$[-$.} & -- &. & .. & .. &.- & --- & $-\ldots$ & .... & --- &..- & -- & -- & -- & .- & & \\
\hline (Cawtoniceras) whiteavesi Reeside & -.. & $\ldots$ & .... & -... & $\ldots$ & & & $\ldots$ & ... & & .... & 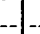 & .. & & & ... & $\ldots$ & $\ldots$ & $x$ & -- &.- &.- &.- & -- & -- & -- & - & -- & --- & ---- & $-\ldots$ & ---- & -- & -- & -- & -- & -- & - & \\
\hline (Maltoniceras) sundancense Reeside & $\ldots$ & إ--. & .... & -.. & $\ldots$ & & & $\ldots$ & -- & & 1 & & & & & & & $-\ldots$ & $x$ & -.. & -- & -- & -- &.- & - & -- & - & -- & $\ldots$ & $-\ldots$ & --- & --- & $\ldots$ & -- & -- & -- & -- & & \\
\hline (Subvertebriceras) canadense Wh & $\ldots$ & $\ldots$ & -... & & $\ldots$ & & & -- & .. & & & & & & & & & 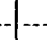 & & & $-\overline{1}$ &.- &.- & -- & -- & -- & -- & -- & --- & --- & $\cdots$ & $-\cdots$ & -- & -- & $=$ & -- & - & & \\
\hline (Vertebriceras) stantoni Reeside & -. & & 1 & & 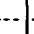 & & & 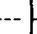 & - & & & & & & & & & d & & -- & $\times$ & -- &.- & -- & - & -- & - & -- & --- & $---\mid$ & $\ldots$ & --- & -- & -- & $x$ & --1 & $\times$ & 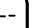 & \\
\hline (V.) haresi $\mathrm{R}$ & $\ldots$ & & $\times 1-$ & & $\cdots$ & & & $\cdots$ & -- & & & & & & & & & & & $-\cdot$ & -- & -- & -- & -- & - & $\ddot{x}$ & -- & -- & --- & $\cdots$ & --- & --- & $\cdots$ & -- & -- & -- & -- & -- & \\
\hline $\begin{array}{l}\text { (Sagntticeras) obtusum Reeside } \\
\text { irossouvria (Poculisphinctes?) cf. G. (P.) trina }\end{array}$ & & & & & & & & & & & & & & & & & & & & & & & & & & & & & & & & & & & & $\cdots$ & & & \\
\hline (Buckman) & & & & & & & & & & & & & & & & & & & & & & -- & & & & $x$ & & -- & $-\cdots$ & $-\cdots$ & --- & $-\cdots$ & & -- & -- & -- & -- & & \\
\hline
\end{tabular}


TABLE 7.-Description of Jurassic (late Callovian and Oxfordian) ammonite localities in the Western Interior region of the United States

\begin{tabular}{|c|c|c|c|}
\hline $\begin{array}{l}\text { Loc. No. } \\
\text { (fig. 1) }\end{array}$ & $\begin{array}{l}\text { USGS Mesozoic } \\
\text { loc. No. }\end{array}$ & $\begin{array}{l}\text { Collector's } \\
\text { field No. }\end{array}$ & Collector, year of collection, description of locality, and stratigraphic assignment \\
\hline 1 & 27641 & -......--- & $\begin{array}{l}\text { W. A. Cobban, R. W. Imlay, and N. F. Sohl, 1960. North side of Burlington Northern } \\
\text { Railway 0.4 km east of Autumn Creek on south edge of Glacier National Park, Marias } \\
\text { Pass quadrangle. Montana. Swift Formation near top of lower member in sandv shale. }\end{array}$ \\
\hline 2 & 30090 & I71-7-20A & $\begin{array}{l}\text { R. W. Imlay and W. O. Ross, } 1971 \text {. Bearpaw Mountains. On ridge east of center of dome } \\
\text { north of Peoples Creek in east-central part of NE } 1 / 4 \mathrm{NE} 1 / 4 \text { sec. } 32 \text {, T. } 29 \mathrm{~N} \text {., R. } 18 \mathrm{E} \text {., } \\
\text { Maddux quadrangle, Blaine County, Mont. Swift Formation, lower part. }\end{array}$ \\
\hline 3 & 30099 & I71-7-19B & $\begin{array}{l}\text { R. W. Imlay and W. O. Ross, 1971. Bearpaw Mountains in western part of Taylor Butte, } \\
\text { SE. cor. sec. } 31, \mathrm{~T} .28 \mathrm{~N} \text {., R. } 19 \text { E., Rattlesnake quadrangle, Blaine County, Mont. Swift } \\
\text { Formation. } 7 \mathrm{~m} \text { above base. }\end{array}$ \\
\hline 4 & 27006 & P57-25 & $\begin{array}{l}\text { B. C. Hearn, Jr., 1957. Bearpaw Mountains northwest of Taylor Butte in NW. cor. NE } 1 / 4, \text {, } \\
\text { NE } 1 / 4 \text { sec. } 11 \text { T. } 28 \text { N., R. } 19 \text { E., Rattlesnake quadrangle, Blaine County, Mont. Swift } \\
\text { Formation, } 10.6 \mathrm{~m} \text { above base. }\end{array}$ \\
\hline 4 & 29277 & I65-72-9B & $\begin{array}{l}\text { W. O. Ross and R. W. Imlay, } 1965 \text {. Bearpaw Mountains } 0.8 \mathrm{~km} \text { northwest of Taylor Butte } \\
\text { in NW } 1 / 4 \text { NE } 1 / 4 \text { sec. } 11, \mathrm{~T} .28 \text { W., R. } 19 \text { E., Rattlesnake quadrangle, Blaine County, Mont. } \\
\text { Swift Formation. } 6-7.6 \mathrm{~m} \text { above base. }\end{array}$ \\
\hline 4 & 29278 & I65-7-29C & $\begin{array}{l}\text { B. C. Hearn, Jr., and others, } 1965 \text {. Bearpaw Mountains, } 0.24 \mathrm{~km} \text { east-northeast from } \\
\text { USGS Mesozoic loc. } 29277 \text { in SE } 1 / 4 \mathrm{SE} / 4 \mathrm{sec} \text {. } 2 \text {, T. } 28 \mathrm{~N} \text {., R. } 19 \text { E., Rattlesnake quad- }\end{array}$ \\
\hline 4 & 30093 & I71-71-19C & $\begin{array}{l}\text { R. W. Imlay and W. O. Ross, } 1971 \text {. Bearpaw Mountains. West side of Taylor Butte in } \\
\text { east-central part of SE } 1 / 4 \mathrm{SE}_{1 / 4} \text { sec. } 2, \mathrm{~T} \text {. } 28 \mathrm{~N} \text {., R. } 19 \mathrm{E} \text {., Rattlesnake quadrangle, Blaine }\end{array}$ \\
\hline 4 & 30098 & I71-7-19A & $\begin{array}{l}\text { R. W. Imlay and W. O. Ross, } 1971 \text {. Bearpaw Mountains. West side of Taylor Butte in } \\
\text { north-central part of NE1/4 NE } 1 / 4 \text { sec. 11, T. } 28 \mathrm{~N} \text {., R. 19 E., Rattlesnake quadrangle, } \\
\text { Blaine County, Mont. Swift Formation, } 6.7 \mathrm{~m} \text { above base. Near same place as USGS } \\
\text { Mesozoic loc. } 29277 \text {. }\end{array}$ \\
\hline 5 & 18761 & - & $\begin{array}{l}\text { M. M. Knechtel and J. B. Reeside, Jr., 1938. Bearpaw Mountains. Suction Creek in south- } \\
\text { central part of sec. } 1 \text {, T. } 28 \mathrm{~N} \text {., R. } 20 \mathrm{E} \text {., Rattlesnake quadrangle, Blaine County, Mont. } \\
\text { Swift Formation, } 6 \mathrm{~m} \text { above base of dark shale member. }\end{array}$ \\
\hline 5 & 19197 & B.P.4 & $\begin{array}{l}\text { R. W. Imlay and H. C. Yingling, Jr., 1944. Bearpaw Mountains. At head of Suction Creek } \\
\text { in south-central part of sec. 1, T. } 28 \text { N., R. } 20 \text { E., Rattlesnake quadrangle, Blaine County, }\end{array}$ \\
\hline 5 & 19199 & B.P.4a & $\begin{array}{l}\text { R. W. Imlay and H. C. Yingling, Jr., 1944. Bearpaw Mountains. Collected about } 46 \mathrm{~m} \text { east } \\
\text { of USGS Mesozoic loc. } 19197 \text { at about same stratigraphic position. }\end{array}$ \\
\hline 5 & 29279 & I65-7-28C & $\begin{array}{l}\text { B. C. Hearn, Jr., W. O. Ross, and R. W. Imlay, 1965. Bearpaw Mountains. On north side } \\
\text { of road in NE } 1 / 4 \mathrm{NW} 1 / 4 \mathrm{NE} 1 / 4 \text { sec. } 12 \text {, T. } 28 \mathrm{~N} \text {., R. } 20 \text { E., Rattlesnake quadrangle, Blaine } \\
\text { County, Mont. Swift Formation, probably } 6-7.6 \mathrm{~m} \text { above base. }\end{array}$ \\
\hline 5 & 30089 & 171-7-18B & W. O. Ross and R. W. Imlay, 1971. Bearpaw Mountains. On north side of Suction Creek \\
\hline 5 & 30094 & 171-7-18D & $\begin{array}{l}\text { W. O. Ross and R. W. Imlay, 1971. Bearpaw Mountains. Same place as USGS Mesozoic loc. } \\
\text { 29279. Swift Formation, 8.5-9.7 m above base. }\end{array}$ \\
\hline 5 & 30095 & I $71-718 \mathrm{C}$ & $\begin{array}{l}\text { W. O. Ross and R. W. Imlay, 1971. Bearpaw Mountains. Same place as USGS Mesozoic loc. } \\
\text { 29279. Swift Formation, } 5.2-6 \mathrm{~m} \text { above base. }\end{array}$ \\
\hline 5 & 30096 & $171-718 \mathrm{~F}$ & $\begin{array}{l}\text { W. O. Ross and R. W. Imlay, 1971. Bearpaw Mountains. Same place as USGS Mesozoic loc. } \\
29279 \text { Swift Formation. } 174-18.3 \text { m above base. }\end{array}$ \\
\hline 5 & 30097 & $171-7-18 \mathrm{E}$ & W. O. Ross and R. W. Imlay, 1971. Bearpaw Mountains. Same place as USGS Mesozoic loc. \\
\hline 6 & 29289 & I65-8-3D & $\begin{array}{l}\text { R. W. Imlay and W. O. Ross, 1965. Little Rocky Mountains. North-central part of Chalk } \\
\text { Butte in center of SE } 1 / 4 \text { SW } 1 / 4 \text { sec. } 6 \text {, T. } 25 \text { N., R. } 26 \text { E., Phillips County, Mont. Swift } \\
\text { Formation, lower } 7.6 \mathrm{~m} \text {. }\end{array}$ \\
\hline 6 & 29290 & $\mathrm{I} 65-8-3 \mathrm{E}$ & $\begin{array}{l}\text { R. W. Imlay and W. O. Ross, } 1965 \text {. Little Rocky Mountains. North side of Chalk Butte in } \\
\text { NW } 1 / 4 \mathrm{SW}_{1 / 4} \mathrm{SE} 1 / 4 \text { sec. } 6, \text { T. } 25 \text { N., R. } 26 \text { E., Phillips County, Mont. Swift Formation, } \\
\text { lower } 9 \mathrm{~m} \text {. }\end{array}$ \\
\hline 6 & 9835 & 28 & $\begin{array}{l}\text { H. R. Bennett and A. J. Collier, 1916. Little Rocky Mountains at east end in sec. 7, } \\
\text { T. } 25 \text { N., R. } 26 \text { E. Phillips County, Mont. Swift Formation. }\end{array}$ \\
\hline 6 & 19207 & L.R. 8 & $\begin{array}{l}\text { R. W. Imlay and H. C. Yingling, Jr., 1944. Little Rocky Mountains at Chalk Butte in sec. } \\
7 \text {, T. } 25 \text { N., R. } 26 \text { E., Phillips County, Mont. Swift Formation, about } 11.5 \mathrm{~m} \text { above base } \\
\text { in soft gray claystone. }\end{array}$ \\
\hline 6 & 19210 & L.R.10 & $\begin{array}{l}\text { R. W. Imlay and H. C. Yingling, Jr., 1944. Little Rocky Mountains on east side of Chalk } \\
\text { Butte in sec. 7, T. } 25 \text { N., R. } 26 \text { E., Phillips County, Mont. Swift Formation, gray shale } \\
\text { about } 11.5 \text { m above base. }\end{array}$ \\
\hline 6 & 19578 & I45-8-8D & $\begin{array}{l}\text { R. W. Imlay and W. G. Saalfrank, 1945. Little Rocky Mountains near west end of Chalk } \\
\text { Butte in sec. } 7, \mathrm{~T} \text {. } 25 \mathrm{~N} \text {., R. } 26 \mathrm{E} \text {., Phillips County, Mont. Swift Formation, about } \\
9-10.5 \mathrm{~m} \text { above base and } 3 \mathrm{~m} \text { below a row of limestone concretions. }\end{array}$ \\
\hline 7 & 19580 & I45-8-5C & $\begin{array}{l}\text { R. W. Imlay and W. G. Saalfrank, } 1945 \text {. Little Rocky Mountains on east side of Matador } \\
\text { Butte in east-central part of sec. 3, T. } 25 \text { N., R. } 26 \text { E., Phillips County, Mont. Swift } \\
\text { Formation, } 4.5 \mathrm{~m} \text { above base. }\end{array}$ \\
\hline 8 & 19205 & L.R.4 & $\begin{array}{l}\text { R. W. Imlay and H. C. Yingling, Jr., 1944. Little Rocky Mountains about } 1.6 \mathrm{~km} \text { southwest } \\
\text { of Landusky in sec. } 32 \text {. T. } 25 \mathrm{~N} \text {., R. } 24 \mathrm{E} \text {., Phillips County, Mont. Swift Formation, from } \\
\text { upper part of gypsiferous shale and } 3 \mathrm{~m} \text { below lowest sandstone bed. }\end{array}$ \\
\hline 9 & 19582 & I45-8-5D & $\begin{array}{l}\text { R. W. Imlay and W. G. Saalfrank. Little Rocky Mountains about 1.6 km east of Zortman on } \\
\text { north side of road. Probably in east-central part of sec. 15, T. } 25 \text { N., R. } 25 \text { E., Phillips } \\
\text { County, Mont. }\end{array}$ \\
\hline 9 & 30102 & I71-7-14B & $\begin{array}{l}\text { W. O. Ross and R. W. Imlay, 1971. Little Rocky Mountains on west side of Zortman Butte } \\
\text { just south of divide in south-central part of NW } 1 / 4 \mathrm{NE} 1 / 4 \text { sec. } 20 \text {, T. } 25 \mathrm{~N} \text {., R. } 25 \mathrm{E} \text {., Phillips } \\
\text { County, Mont. Swift Formation, from upper } 6 \mathrm{~m} \text { of claystone unit, which is about } 9 \mathrm{~m} \\
\text { thick. }\end{array}$ \\
\hline 10 & 18728 & 4 & $\begin{array}{l}\text { J. B. Reeside, Jr., M. M. Knechtel, and S. W. Hobbs, } 1939 \text {. Little Rocky Mountains at } \\
\text { Morrison Butte in SE } 1 / 4 \text { sec. } 12 \text {, T. } 24 \text { N., R. } 24 \text { E., Phillips County, Mont. Swift } \\
\text { Formation, loose fragments of ammonites collected in shale about } 4.5 \text { m above contact } \\
\text { with limestone. }\end{array}$ \\
\hline
\end{tabular}


TABLE 7.-Description of Jurassic (late Callovian and Oxfordian) ammonitelocalities in the Western Interior region of the United States -Continued

\begin{tabular}{|c|c|c|c|}
\hline $\begin{array}{l}\text { Loc. No. } \\
\text { (fig. 1) }\end{array}$ & $\begin{array}{l}\text { USGS Mesozoic } \\
\text { loc. No. }\end{array}$ & $\begin{array}{l}\text { Collector's } \\
\text { field No. }\end{array}$ & Collector, year of collection, description of locality, and stratigraphic assignment \\
\hline 10 & 19204 & L.R.2 & $\begin{array}{l}\text { R. W. Imlay and H. C. Yingling, Jr., 1944. Little Rocky Mountains at Morrison Butte in } \\
\text { sec. } 12 \text {, T. } 24 \text { N., R. } 24 \text { E., Phillips County, Mont. Swift Formation, from gypsiferous } \\
\text { shale just below upper sandstone member. }\end{array}$ \\
\hline 10 & 29288 & $\mathrm{I} 65-8-2 \mathrm{~A}$ & $\begin{array}{l}\text { R. W. Imlay and W. O. Ross, } 1965 \text {. Little Rocky Mountains at Morrison Butte in west- } \\
\text { central part of SW } 1 / 4 \text { sec. } 7 \text {, T. } 24 \text { N., R. } 25 \text { E., Phillips County, Mont. Swift Formation, } \\
\text { basal } 3 \text { m of lower siltstone member. }\end{array}$ \\
\hline 10 & 30101 & I71-7-16D & $\begin{array}{l}\text { R. W. Imlay and W. O. Ross, } 1971 \text {. Little Rocky Mountains at Morrison Butte in SW } 1 / 4 \text {. } \\
\text { NW } 1 / 4 \mathrm{SW}_{1 / 4} \mathrm{sec} \text {. } 7 \text {, } 24 \mathrm{~N} \text {., R. } 25 \mathrm{E} \text {., Phillips County, Mont. Swift Formation, float } \\
\text { from lower } 6 \mathrm{~m} \text { of claystone. }\end{array}$ \\
\hline 11 & 19579 & I45-8-7B & $\begin{array}{l}\text { R. W. Imlay and W. G. Saalfrank, 1945. Little Rocky Mountains on south side Camp Creek } \\
\text { Butte in SW. cor. sec. 36, T. } 25 \text { N., R. } 25 \text { E., Phillips County, Mont. Swift Formation, } \\
6-7.6 \mathrm{~m} \text { above base. }\end{array}$ \\
\hline 11 & 30083 & I71-7-17D & $\begin{array}{l}\text { R. W. Imlay and W. O. Ross, 1971. Little Rocky Mountains on south side of Camp Creek } \\
\text { Butte in SW1/4 SW } 1 / 4 \text { sec. } 36 \text {, T. } 25 \mathrm{~N} \text {., R. } 25 \mathrm{E} \text {., Phillips County, Mont. Swift Formation, } \\
\text { about } 10.6 \mathrm{~m} \text { above base in a white limestone lentil. }\end{array}$ \\
\hline 11 & 30084 & I71-7-17E & $\begin{array}{l}\text { R. W. Imlay and W. O. Ross, 1971. Little Rocky Mountains at same location as USGS } \\
\text { Mesozoic loc. } 30083 \text {. Swift Formation, } 6-12 \mathrm{~m} \text { above base. }\end{array}$ \\
\hline 11 & 30085 & $\mathrm{I} 71-7-17 \mathrm{~F}$ & $\begin{array}{l}\text { W. O. Ross, 1971. Little Rocky Mountains at Camp Creek Butte. About } 122 \mathrm{~m} \text { northwest } \\
\text { of USGS Mesozoic locs. } 30083 \text { and } 30084 \text { on west side of gulch in } \mathrm{SW} 1 / 4 \mathrm{SW} 1 / 4 \text { sec. } 36 \text {, } \\
\text { T. } 25 \mathrm{~N} \text {., R. } 25 \text { E., Phillips County, Mont. Swift Formation, Cardiceras-bearing beds } \\
\text { about } 12 \mathrm{~m} \text { above orange beds at USGS Mesozoic loc. } 30086 \text {, which mark top of range }\end{array}$ \\
\hline 11 & 30086 & I71-7-17G & $\begin{array}{l}\text { R. Imlay and W. O. Ross, 1971. Little Rocky Mountains at same location as } \\
\text { USGS Mesozoic loc. } 30083 \text {. Swift Formation, orange concretionary beds about } 20 \mathrm{~m}\end{array}$ \\
\hline 12 & 9832 & 23 & H. R. Bennett for A. J. Collier, 1916. Little Rocky Mountains at East Coburn Butte. \\
\hline 12 & 19577 & I45-8-7D & $\begin{array}{l}\text { R. W. Imlay and W. G. Saalfrank, } 1945 \text {. Little Rocky Mountains at southwest end of west } \\
\text { Coburn Butte in sec. 33, T. } 25 \text { N. R. } 26 \text { E. Phillips County Mont. Swift Formation. }\end{array}$ \\
\hline 12 & 30103 & I71-7-15B & $\begin{array}{l}\text { R. W. Imlay and W. O. Ross, } 1971 \text {. Little Rocky Mountains on south side of West Coburn } \\
\text { Butte in NW } 1 / 4 \text { SW } 1 / 4 \text { NE } 1 / 4 \text { sec. 33, T. } 25 \text { N., R. } 26 \text { E., Phillips County, Mont. Swift } \\
\text { Formation } 12-18 \mathrm{~m} \text { above base of claystone unit at base of formation. }\end{array}$ \\
\hline 13 & 19171 & B.S. 2 & $\begin{array}{l}\text { R. W. Imlay and H. C. Yingling, Jr., 1944. Big Snowy Mountains, } 0.8 \mathrm{~km} \text { south of Heath } \\
\text { in sec. } 2 \text {, T. } 14 \mathrm{~N} \text {. R. } 19 \mathrm{E} \text {., Fergus County, Mont. Swift Formation, Float on upper } \\
4.5 \mathrm{~m} \text { of gray shale at top of the Rierdon Formation. }\end{array}$ \\
\hline 14 & 19173 & B.S.15 & $\begin{array}{l}\text { R. W. Imlay, } 1944 \text {. Big Snowy Mountains. on north side of road and } 2.4 \mathrm{~km} \text { east of Piper } \\
\text { in northern part of sec. } 14, \mathrm{~T} \text {. } 14 \mathrm{~N} \text {., R. } 20 \mathrm{E} \text {., Fergus County, Montana. Swift Formation, } \\
\text { about } 7.6 \mathrm{~m} \text { above sandy limestone bench and } 6 \mathrm{~m} \text { below upper sandstone. }\end{array}$ \\
\hline 14 & 19179 & B.S. 3 & $\begin{array}{l}\text { R. W. Imlay and H. C. Yingling, Jr., 1944. Big Snowy Mountains, } 1.6 \mathrm{~km} \text { southwest of } \\
\text { Piper on southwest corner of scarp in SE } 1 / 4 \text {, sec. } 17, \mathrm{~T} \text {. } 14 \mathrm{~N} \text {., R. } 20 \mathrm{E} \text {., Fergus County, } \\
\text { Mont. Swift Formation, float lying on } 10 \mathrm{~m} \text { of fissile shale and nodular limestone in } \\
\text { basal part of Rierdon Formation. Ammonite probably derived from } 3 \mathrm{~m} \text { of ledgy } \\
\text { limestone at base of the Swift. }\end{array}$ \\
\hline 14 & 22461 & & $\begin{array}{l}\text { R. W. Imlay, 1950. Big Snowy Mountains southwest of Piper at same place as USGS } \\
\text { Mesozic loc. } 19179 \text {. Swift Formation, basal shale member, } 4.5 \mathrm{~m} \text { above top of Rierdon } \\
\text { Formation. }\end{array}$ \\
\hline 14 & 22462 & & $\begin{array}{l}\text { R. W. Imlay, 1950. Big Snowy Mountains southwest of Piper at same place as USGS } \\
\text { Mesozoic loc. 19179. Swift Formation, basal shale member; fossils obtained from con- } \\
\text { cretions } 3 \mathrm{~m} \text { above top of Rierdon Formation. }\end{array}$ \\
\hline 15 & 19611 & I $45-8-3 \mathrm{~A}$ & $\begin{array}{l}\text { R. W. Imlay and W. G. Saalfrank, 1945. South side of Big Snowy Mountains. } 1.6 \mathrm{~km} \text { west } \\
\text { of Cameron Creek near line between secs. } 28 \text { and } 33 \text {, T. } 11 \text { N., R. } 21 \text { E., Golden Valley }\end{array}$ \\
\hline 16 & 31720 & $60-G V-3$ & $\begin{array}{l}\text { Marvin Kaufmann, } 1961 \text {. East side of Pryor Mountains on Grapevine Creek near the } \\
\text { Bighorn River; about } 64 \mathrm{~km} \text { southwest of Billings in sec. 6, T. } 6 \text { S., R. } 31 \text { E., Bighorn } \\
\text { County, Mont. Swift Formation. }\end{array}$ \\
\hline 17 & 19612 & I45-7-4A & $\begin{array}{l}\text { R. W. Imlay and W. G. Saalfrank, 1945. West side of Pryor Mountains in southwest corner } \\
\text { of Red dome in sec. 19, T. } 7 \text { S., R. } 24 \text { E., Carbon County, Mont. Swift Formation, fossils } \\
\text { from } 6 \mathrm{~m} \text { of silty clavstone } 6 \mathrm{~m} \text { above the base of the formation }\end{array}$ \\
\hline 17 & 27722 & -......... & N. F. Sohl and R. W. Imlay, 1960. Pryor Mountains. West flank of Red dome in sec. 19, \\
\hline 17 & 29542 & I66-7-8D & $\begin{array}{l}\text { R. W. Imlay, W. O. Ross, and Mario Suarez, 1966. Pryor Mountains. West flank of Red } \\
\text { dome at same place and stratigraphic position as USGS Mesozoic loc. } 19612 \text {. }\end{array}$ \\
\hline 17 & 29769 & I68-7-13A & $\begin{array}{l}\text { W. } 0 \text {. Ross and R. W. Imlay, 1968. Pryor Mountains. West flank of Red dome from } 0.4- \\
0.8 \mathrm{~km} \text { north of road from Bridger to Bowler in NE } 1 / 4 \mathrm{sec} 19 \text {, T. } 7 \mathrm{~S} \text {., R. } 24 \mathrm{E} \text {., Carbon } \\
\text { County, Mont. Swift Formation, from } 6 \mathrm{~m} \text { of silty claystone whose base is } 6 \mathrm{~m} \text { above } \\
\text { base of the formation. }\end{array}$ \\
\hline 17 & 29770 & I68-7-13B & $\begin{array}{l}\text { W. O. Ross and R. W. Imlay, 1968. Pryor Mountains. West flank of Red dome about } 0.4 \mathrm{~km} \\
\text { north of road from Bridger to Bowler in SW } 1 / 4 \mathrm{NE} 1 / 4 \text { sec. } 19 \text {, T. } 7 \mathrm{~S} \text {., R. } 24 \mathrm{E} \text {., Carbon } \\
\text { County, Mont. Swift Formation, sandy beds overlying the } 6 \mathrm{~m} \text { of claystone listed under } \\
\text { USGS Mesozoic locs. } 19612.29542 \text { and } 29769 \text {. }\end{array}$ \\
\hline 18 & 30087 & I71-7-6C & $\begin{array}{l}\text { R. W. Imlay, 1971. Pryor Mountains. South flank of Red dome near center } \mathrm{SW} 1 / 4 \mathrm{sec} \text {. } 28 \text {, } \\
\text { T. } 7 \mathrm{~S} \text {, R. } 24 \mathrm{E} \text {; about } 0.4 \mathrm{~km} \text { southwest of confluence of two gulches, Carbon County, } \\
\text { Mont. Swift Formation from top of claystone unit just below upper cliff-forming } \\
\text { sandstone. }\end{array}$ \\
\hline 18 & 30091 & I71-7-10A & $\begin{array}{l}\text { W. O. Ross and R. W. Imlay, 1971. Pryor Mountains. South flank of Red dome in SW } 1 / 4 \\
\text { SE } 1 / 4 \text { sec. } 28 \text {, T. } 7 \text { S., R. } 24 \text { E., Carbon County, Mont. Swift Formation, lower part, about } \\
6 \mathrm{~m} \text { below bed containing large oysters, } 21 \mathrm{~m} \text { below base of upper sandstone unit and } \\
8.2 \mathrm{~m} \text { above highest occurrence of Gryphaea in the upper part of the Rierdon } \\
\text { Formation. }\end{array}$ \\
\hline
\end{tabular}


TABLE 7:-Description of Jurassic (late Callovian and Oxfordian) ammonite localities in the Western Interior region of the United States -Continued

\begin{tabular}{|c|c|c|c|}
\hline $\begin{array}{c}\text { Loc. No. } \\
\text { (fig. 1) }\end{array}$ & $\begin{array}{l}\text { USGS Mesozoic } \\
\text { loc. No. }\end{array}$ & $\begin{array}{l}\text { Collector's } \\
\text { field No. }\end{array}$ & Collector, year of collection, description of locality, and stratigraphic assignment \\
\hline 18 & 30092 & I71-7-10B & $\begin{array}{l}\text { W. O. Ross and R. W. Imlay, 1971. Pryor Mountains at same place as USGS Mesozoic loc. } \\
30091 \text { but about } 3 \mathrm{~m} \text { higher. }\end{array}$ \\
\hline 19 & 30395 & I73-7-11B & $\begin{array}{l}\text { W. A. Ross and R. W. Imlay, 1973. Caribou Range. Roadcut on north side of McCoy Creek } \\
\text { near center of } \mathrm{E}^{1 / 2} \mathrm{SW} 1 / \mathrm{4} \text { sec. } 6 \text {, T. } 3 \mathrm{~S} \text {., R. } 46 \mathrm{E} \text {., Irwin quadrangle, Bonneville County, } \\
\text { Idaho. Stump Formation, Redwater Member. }\end{array}$ \\
\hline 20 & 31349 & I76-9-11 & $\begin{array}{l}\text { R. W. Imlay and G. N. Pipiringos, } 1976 \text {. On ridge west of Dutch George Creek in NW } 1 / 4 \text {. } \\
\text { NE } 1 / 4 \mathrm{SE}^{1 / 4} \text { sec. 3, T. } 25 \mathrm{~N} \text {., R. } 115 \text { W., Fort Hill quadrangle, Lincoln County, Wyo. } \\
\text { Stump Formation, Redwater Member, } 15 \mathrm{~m} \text { below Ephraim Conglomerate and } 30 \mathrm{~m} \\
\text { above base of Redwater Member. }\end{array}$ \\
\hline 21 & 29673 & 7U-1-FC2 & $\begin{array}{l}\text { G. N. Pipiringos and B. H. Helming, 1962. Near Manilla in } \mathrm{SW}^{1 / 4} \mathrm{SW}^{1 / 4} \mathrm{SW} 1 / 4 \mathrm{sec} .27 \text {, } \\
\text { T. } 3 \text { N., R. } 69 \text { W., Manilla quadrangle, in northeast Utah. Stump Formation, Redwater } \\
\text { Member, basal } 3 \mathrm{~m} \text {. }\end{array}$ \\
\hline 22 & 29677 & $7 \mathrm{U}-2-\mathrm{FC} 2$ & $\begin{array}{l}\text { G. N. Pipiringos and B. H. Helming, 1962. Little Brush Creek in } \mathrm{SE} 1 / 4 \text { sec. } 3, \mathrm{~T} .3 \mathrm{~S} \text {, } \\
\text { R. } 22 \text { E., about } 20 \mathrm{~km} \text { north-northeast of Vernal, Utah. Stump Formation, Redwater } \\
\text { Member, uppermost } 9 \mathrm{~m} \text {. }\end{array}$ \\
\hline 23 & 6283 & ---------- & $\begin{array}{l}\text { Robert Forester, } 1909 \text {. Stanaker Draw, probably sec. } 8, \text { T. } 2 \text { N., R. } 22 \text { E., north of Vernal, } \\
\text { Uintah County, Utah. Stump Formation, Redwater Member. }\end{array}$ \\
\hline 24 & 11253 & 2 & $\begin{array}{l}\text { J. B. Reeside, Jr., 1922. Island Park, } 1.6 \mathrm{~km} \text { below the C. Ruple Ranch, } 40 \mathrm{~km} \text { northeast } \\
\text { of Vernal, Utah; same distance south of Wyoming border; and } 9.6 \mathrm{~km} \text { west of Colorado } \\
\text { border, Uintah County, Utah. Stump Formation, Redwater Member. }\end{array}$ \\
\hline 25 & 11256 & 5 & $\begin{array}{l}\text { J. B. Reeside, Jr., } 1922 \text {. Dinosaur Quarry, } 10 \mathrm{~km} \text { north of Jensen, Uintah County, Utah. } \\
\text { Stump Formation, Redwater Member. }\end{array}$ \\
\hline 25 & 8748 & 188 & $\begin{array}{l}\text { Earl Douglas, 1913. Dinosaur Quarry north of Jensen, Utah. Stump Formation, Redwater } \\
\text { Member. }\end{array}$ \\
\hline 27 & 27730 & $\mathrm{C} 87$ & $\begin{array}{l}\text { George Pipiringos, 1962. From small tributary on east side of Little Snake River, } 6.4 \mathrm{~km} \\
\text { above its junction with the Yampa River in NW } 1 / 4 \text { sec. } 4 . \mathrm{T} \text {. } 6 \mathrm{~N} \text {., R. } 98 \mathrm{~W} \text {., Lone } \\
\text { Mountain quadrangle, Moffat County, Colo. Stump Formation, Redwater Member. } \\
\text { Float from } 6 \mathrm{~m} \text { above base of member. Probably derived from limestone ledge } 11.5 \mathrm{~m} \\
\text { above base of member. } \\
\text { J. C. Wright and Jacob Nir, 1960. Lily Park near NW. cor. sec. } 28 \text {, T. } 6 \text { N., R. } 99 \text { W., Moffat } \\
\text { County, Colo. Stump Formation, Redwater Member. }\end{array}$ \\
\hline 28 & 26717 & JsSM2 & $\begin{array}{l}\text { R. L. Rioux, 1957. Bighorn Basin. East flank of Little Sheep Mountain in NW } 1 / 4 \mathrm{sec} .22 \text {, } \\
\text { T. } 56 \mathrm{~N} ., \mathrm{R} \text {. 95 W.; Big Horn County, Wyo. Sundance Formation, Redwater Shale } \\
\text { Member, } 6 \mathrm{~m} \text { (?) below top. }\end{array}$ \\
\hline 28 & 29751 & I68-7-12B & $\begin{array}{l}\text { W. O. Ross. and } R \text {. W. Imlay, 1968. Bighorn Basin, west flank of Little Sheep Mountain } \\
\text { in west-central part of SE } 1 / 4 \text { sec. } 33 \text {, T. } 56 \text { N., R. } 95 \text { W., Big Horn County, Wyo. Sun- } \\
\text { dance Formation, Redwater Shale Member, about } 10.6 \text { m above base. }\end{array}$ \\
\hline 28 & 29755 & I68-7-12C & $\begin{array}{l}\text { R.W. Imlay and W. O. Ross, 1968. Bighorn Basin. Northwest end of Little Sheep Mountain } \\
\text { in NW } 1 / 4 \text { sec. 2, T. } 56 \text { N., R. } 95 \text { W., Big Horn County, Wyo. Sundance Formation, } \\
\text { Redwater Shale Member, about } 10.6 \mathrm{~m} \text { above base, } 11 \mathrm{~m} \text { below an oyster bed, and } 23 \mathrm{~m} \\
\text { below a sandstone cliff. }\end{array}$ \\
\hline 28 & 31744 & $-2-2--$ & $\begin{array}{l}\text { Emmett Evanoff, 1978. Bighorn Basin, northwest flank of Little Sheep Mountain, NW } 1 / 4,4 \text {. } \\
\text { NW } 1 / 4 \text { sec. } 21 \text {, T. } 56 \text { N., R. } 95 \text { W., Lovell Lakes } 71 / 2 \text { min. quadrangle, Big Horn County, } \\
\text { Wyo. Sundance Formation, Redwater Shale Member, fossils in concretions in olive- } \\
\text { gray siltstone about } 13 \mathrm{~m} \text { above base of member. }\end{array}$ \\
\hline 28 & 31750 & 2 & $\begin{array}{l}\text { C. F. Vondra and students, 1968. Bighorn Basin. SE } 1 / 4 \text { sec. } 3 \text {, T. } 55 \text { N., R. } 95 \text { W., Big Horn } \\
\text { County, Wyo. Sundance Formation, claystone in Redwater Shale Member. }\end{array}$ \\
\hline 28 & 31754 & 6 & $\begin{array}{l}\text { C. F. Vondra and students, 1968. Bighorn Basin. SE } 1 / 4 \text { sec. } 3 \text {, T. } 55 \text { N., R. } 95 \text { W., Big Horn } \\
\text { County, Wyo. Sundance Formation, Redwater Shale Member. }\end{array}$ \\
\hline 29 & 31751 & 2 & $\begin{array}{l}\text { C. F. Vondra and students, } 1968 \text {. Bighorn Basin. NW } 1 / 4 \text { sec. } 1 \text {, T. } 54 \text { N., R. } 95 \text { W., Big Horn } \\
\text { County, Wyo. Sundance Formation, Siltstone in Redwater Shale Member. }\end{array}$ \\
\hline 29 & 31752 & 3 & $\begin{array}{l}\text { C. F. Vondra and students, } 1968 \text {. Bighorn Basin. NW } 1 / 4 \text { sec. } 1, \text { T. } 54 \text { N., R. } 95 \text { W., Big Horn } \\
\text { County, Wyo. Sundance Formation, Redwater Shale Member. }\end{array}$ \\
\hline 29 & 31753 & 5 & $\begin{array}{l}\text { C. F. Vondra and students, } 1968 \text {. Bighorn Basin. NW } 1 / 4 \text { sec. } 1 \text {, T. } 54 \text { N., R. } 95 \text { W., Big Horn } \\
\text { County, Wyo. Sundance Formation, Redwater Shale Member. }\end{array}$ \\
\hline 29 & 29759 & I68-7-9A & $\begin{array}{l}\text { W. O. Ross, } 1968 \text {. Bighorn Basin at northwest end of Spence dome in SE } 1 / 4 \text { sec. } 25 \text {, } \\
\text { T. } 55 \text { N., R. } 95 \text { W., Big Horn County, Wyo. Sundance Formation, Redwater Shale }\end{array}$ \\
\hline 30 & 30118 & $171-7-9 \mathrm{C}$ & $\begin{array}{l}\text { R. W. Imlay and W. O. Ross, } 1971 \text {. Bighorn Basin. NW } 1 / 4 \mathrm{NE}^{1 / 4} \text { sec. } 33 \text {, T. } 54 \mathrm{~N} \text {., R. } 94 \mathrm{~W} \text {., } \\
\text { Sheep Canyon quadrangle, Big Horn County, Wyo. Sundance Formation, Redwater } \\
\text { Shale Member, about } 14 \mathrm{~m} \text { above top of Hulett Sandstone Member of Sundance and } \\
9 \mathrm{~m} \text { below bed containing big oysters. }\end{array}$ \\
\hline 31 & 29752 & I68-7-7D & $\begin{array}{l}\text { W. O. Ross, 1968. Bighorn Basin at head of gulch in NE } 1 / 4 \text { sec. } 22 \text {, T. } 53 \text { N., R. } 93 \text { W., Big } \\
\text { Horn County, Wyo. Sundance Formation, Redwater Shale Member, about } 11 \text { m above }\end{array}$ \\
\hline 31 & 29754 & I68-7-6B & $\begin{array}{l}\text { W. O. Ross and R. W. Imlay, 1968. Bighorn Basin in NE. cor. sec. } 33 \text {, T. } 53 \text { N., R. } 93 \text { W., } \\
\text { Big Horn County, Wyo. Sundance Formation, Redwater Shale Member, about } 27.4 \mathrm{~m}\end{array}$ \\
\hline 31 & 29765 & I68-7-10A & $\begin{array}{l}\text { R. W. Imlay and W. O. Ross, } 1968 \text {. Bighorn Basin at south end of Big Sheep anticline in } \\
\text { south-central part sec. } 27 \text {, T. } 53 \text { N., R. } 93 \text { W., Big Horn County, Wyo. Sundance } \\
\text { Formation, Redwater Shale Member, about } 20 \mathrm{~m} \text { above top of Hulett Sandstone Mem- } \\
\text { ber of Sundance and } 8 \mathrm{~m} \text { below oyster bed. }\end{array}$ \\
\hline 31 & 31755 & 8 & $\begin{array}{l}\text { C. F. Vondra and students, 1968. Bighorn Basin. NE } 1 / 4 \text { sec. } 33, \text { T. } 53 \text { N., R. } 93 \text { W., Big Horn } \\
\text { County, Wyo. Sundance Formation, Redwater Shale Member. }\end{array}$ \\
\hline 32 & 29753 & I68-7-20B & $\begin{array}{l}\text { R. W. Imlay and W. O. Ross, } 1968 \text {. Bighorn Basin in NE } 1 / 4 \text { sec. } 10, \text { T. } 47 \text { N., R. } 89 \text { W., } \\
\text { Washakie County, Wyo. Sundance Formation, Redwater Shale Member, about } 14 \mathrm{~m} \\
\text { above base. }\end{array}$ \\
\hline 33 & 30104 & $171-7-31 \mathrm{~A}$ & $\begin{array}{l}\text { W. O. Ross and R. W. Imlay, 1971. Wind River Basin, Baldwin Creek area, about } 8 \mathrm{~km} \\
\text { west-northwest of Lander, NE } 1 / 4 \text { sec. } 16 \text {, T. } 33 \text { N., R. } 100 \text { W., Fremont County, Wyo. } \\
\text { Sundance Formation, Redwater Shale Member from } 9-15 \text { m above base. }\end{array}$ \\
\hline
\end{tabular}


TABLE 7.-Description of Jurassic (late Callovian and Oxfordian) ammonitelocalities in the Western Interiorregion of the United States -Continued

\begin{tabular}{|c|c|c|c|}
\hline $\begin{array}{l}\text { Loc. No. } \\
\text { (fig. 1) }\end{array}$ & $\begin{array}{l}\text { USGS Mesozoic } \\
\text { loc. No. }\end{array}$ & $\begin{array}{l}\text { Collector's } \\
\text { field No. }\end{array}$ & Collector, year of collection, description of locality, and stratigraphic assignment \\
\hline 34 & 21090 & -........... & $\begin{array}{l}\text { R. M. Thompson, 1948. Wind River Basin about } 6.4 \mathrm{~km} \text { north-northeast of Lander in } \\
\text { NW }{ }^{1 / 4} \mathrm{NW}^{1 / 4} \text { sec. } 19, \mathrm{~T} \text {. } 2 \text { E., R. } 2 \text { E., Fremont County, Wyo. Sundance Formation, } \\
\text { Redwater Shale Member. }\end{array}$ \\
\hline 35 & 28400 & $1-15 F \# 2$ & $\begin{array}{l}\text { G. N. Pipiringos, 1961. Wind River Basin on Johnson Ranch in NW } 1 / 4 \mathrm{sec} .13, \mathrm{~T} .31 \mathrm{~N} . \text {, } \\
\text { R. } 98 \text { W., Fremont County, Wyo. Sundance Formation, Redwater Shale Member } 24 \mathrm{~m} \\
\text { above base. }\end{array}$ \\
\hline 36 & 11179 & 111 & $\begin{array}{l}\text { W. T. Lee, 1922. Whiskey Gap, in northwest corner of Carbon County, Wyo. Sundance } \\
\text { Formation, Redwater Shale Member. }\end{array}$ \\
\hline 36 & 24736 & .... & $\begin{array}{l}\text { R. G. Hubbell, } 1953 \text {. Western Ferris Mountains in W } 1 / 2 \text { sec. } 11, T .27 \text { N., R. } 88 \text { W., } \\
\text { northwest corner of Carbon County, Wyo. Sundance Formation, Redwater Shale } \\
\text { Member, about } 4.5 \text { m above base. }\end{array}$ \\
\hline 37 & 2386 & $-\cdots---$ & $\begin{array}{l}\text { Charles Schuchert, 1899. Near Grand Canyon of the North Platte River, south of Alcova, } \\
\text { Natrona County. Wyo. Sundance Formation. Redwater Shale Member. }\end{array}$ \\
\hline 37 & 31390 & $\mathrm{I} 77-6-20 \mathrm{~A}$ & $\begin{array}{l}\text { G. N. Pipiringos, } 1977 \text {. On ridge northeast of Cottonwood Creek near Alcova Reservoir in } \\
\text { east-central part of SW } 1 / 4 \mathrm{NW}^{1 / 4} \text { sec. } 1, \mathrm{~T} \text {. } 29 \mathrm{~N} \text {., R. } 83 \text { W., Alcova quadrangle, Wyo. } \\
\text { Sundance Formation, Redwater Shale Member, float near top of siltstone unit probably } \\
\text { derived from lower shale. }\end{array}$ \\
\hline 37 & 31391 & I77-6-20B & $\begin{array}{l}\text { Knut Anderson, } 1977 \text {. About } 0.16 \mathrm{~km} \text { northwest of USGS Mesozoic loc. } 31390 \text { in same } \\
\text { ridge near Alcova Reservoir, Wyo. Sundance Formation, Redwater Shale Member, } \\
\text { float } 12-15 \mathrm{~m} \text { above base of member. }\end{array}$ \\
\hline 38 & 25384 & & $\begin{array}{l}\text { J. D. Love, 1948. Core at depth of } 773 \mathrm{ft}(236 \mathrm{~m}) \text { in Wasatch Oil Company's } 25 \text { Swan Land } \\
\text { and Livestock No. } 1 \text { test well. NE } 1 / 4 \mathrm{NW}^{1 / 4} \mathrm{NE}^{1 / 4} \text { sec. } 25 \text {, T. } 24 \mathrm{~N} \text {., R } 76 \text { W., Albany } \\
\text { County, Wyo. Sundance Formation, Redwater Shale Member. }\end{array}$ \\
\hline 39 & 30315 & I72-8-2A & $\begin{array}{l}\text { W. O. Ross and R. W. Imlay, } 1972 \text {. About } 61 \mathrm{~m} \text { east of road, in south-central part of } \mathrm{SE}^{1 / 4} 4 \\
\text { SW } 1 / 4 \text { sec. } 13 \text {, T. } 27 \mathrm{~N} \text {., R. } 77 \text { W., Chalk Hills quadrangle, Albany County, Wyo. } \\
\text { Sundance Formation, Redwater Shale Member, about } 3 \mathrm{~m} \text { below ledge and } 6.7 \mathrm{~m} \text { below } \\
\text { base of Windy Hill Sandstone Member of Sundance. }\end{array}$ \\
\hline 40 & 30329 & I72-7-31B & $\begin{array}{l}\text { R. W. Imlay, 1972. NW } 1 / 4 \text { SW } 1 / 4 \text { SW } 1 / 4 \text { sec. } 15 \text {, T. } 26 \mathrm{~N} \text {, R. } 76 \mathrm{~W} \text {., Boot Hill quadrangle } \\
\text { Albany County, Wyo. Sundance Formation, Redwater Shale Member, } 2 \mathrm{~m} \text { below top } \\
\text { of lower shale unit and } 13.4 \mathrm{~m} \text { above base of member. }\end{array}$ \\
\hline 41 & 24734 & --------- & $\begin{array}{l}\text { R. G. Hubbell, } 1953 \text {. SW } 1 / 4 \text { sec. } 27 \text {, T. } 26 \text { N., R. } 80 \text { W., Carbon County, Wyo. Sundance } \\
\text { Formation, Redwater Shale Member, about } 3 \mathrm{~m} \text { above base. }\end{array}$ \\
\hline 42 & 30321 & $\mathrm{I} 72-8-6 \mathrm{~B}$ & $\begin{array}{l}\text { W. O. Ross, } 1972 \text {. Freezeout Mountains. Center of SE } 1 / 4 \text { sec. 3, T. } 25 \text { N., R. } 79 \text { W., Cameron } \\
\text { Creek quadrangle, Carbon County, Wyo. Sundance Formation, Redwater Shale Mem- } \\
\text { ber, float from near base of shale unit. }\end{array}$ \\
\hline 43 & 28401 & FC- $\# 1$ & $\begin{array}{l}\text { G. H. Pipiringos, } 1961 \text {. Young Ranch in SW } 1 / 4 \text { sec. } 1 \text {, T. } 24 \text { N., R. } 81 \text { W., Carbon County, } \\
\text { Wyo. Sundance Formation, Redwater Shale Member, } 29 \text { m above base. }\end{array}$ \\
\hline 43 & 24735 & $\# 3$ & $\begin{array}{l}\text { R. G. Hubbell, } 1953 \text {. Watkins Draw in NW } 1 / 4 \text { sec. } 11 \text {, T. } 24 \text { N., R. } 81 \text { W., Carbon County, } \\
\text { Wyo. Sundance Formation, Redwater Shale Member, about } 6.7 \mathrm{~m} \text { above base. }\end{array}$ \\
\hline 43 & 31381 & I77-6-13A & $\begin{array}{l}\text { G. N. Pipiringos and R. W. Imlay, 1977. South side of Watkins Draw in Difficulty quad- } \\
\text { rangle. SW } 1 / 4 \mathrm{SE}^{1 / 4} \mathrm{SW} \text { i/4 sec. } 1 \text {, T. } 24 \mathrm{~N} \text {., R. } 81 \mathrm{~W} \text {. Carbon County, Wyo. Sundance } \\
\text { Formation, Redwater Shale Member, float } 21 \mathrm{~m} \text { above base of member. Probably } \\
\text { derived from a coquinoid sandstone ledge } 15 \mathrm{~m} \text { above base of lower shale. }\end{array}$ \\
\hline 43 & 31382 & I77-6-13B & $\begin{array}{l}\text { R. W. Imlay and G. N. Pipiringos, } 1977 \text {. Watkins Draw on section line } 305 \mathrm{~m} \text { due south } \\
\text { of NE. cor. sec. } 10 \text {, T. } 24 \text { N., R. } 81 \mathrm{~W} \text {, T. E. Ranch quadrangle, Carbon County, Wyo. } \\
\text { Sundance Formation, Redwater Shale Member, float from } 6-7.6 \mathrm{~m} \text { above base. }\end{array}$ \\
\hline 44 & 30318 & I72-8-4C & $\begin{array}{l}\text { R. W. Imlay, 1972. SE } 1 / 4 \mathrm{NE}^{1 / 4} \text { sec. } 19, \mathrm{~T} .24 \mathrm{~N} \text {., R. } 80 \mathrm{~W} \text {., Difficulty quadrangle, Carbon } \\
\text { County, Wyo. Sundance Formation, Redwater Shale Member, about } 15 \mathrm{~m} \text { below the } \\
\text { Windy Hill Sandstone Member of Sundance in a shale unit. }\end{array}$ \\
\hline 44 & 31715 & - & $\begin{array}{l}\text { Collector unknown (see Reeside, } 1919, \text { p. } 8 \text { and 9). Difficulty Canyon in southwestern part } \\
\text { of T. } 24 \text { N., R. } 80 \text { W., Carbon County, Wyo. Sundance Formation, Redwater Shale } \\
\text { Member. }\end{array}$ \\
\hline 45 & 30306 & I $72-7-30 \mathrm{~A}$ & $\begin{array}{l}\text { R. W. Imlay and W. O. Ross, } 1972 \text {. Freezeout Mountains, southwest, in NW } 1 / 4 \mathrm{NW} 1 / 4 \text { sec. } \\
25 \text {, T. } 24 \text { N., R. } 80 \text { W. Windy Hill quadrangle, Carbon County, Wyo. Sundance For- } \\
\text { mation, Redwater Shale Member, from } 1 \text { m ledge which is } 5 \mathrm{~m} \text { below sandstone ledge } \\
\text { in upper part of lower shale unit. }\end{array}$ \\
\hline 45 & 24776 & 6 & $\begin{array}{l}\text { R. G. Hubbell, } 1953 \text {, Freezeout Mountain, in NE } 1 / 4 \text {, sec. } 26, \mathrm{~T} .24 \mathrm{~N}, \mathrm{R} .80 \mathrm{~W} . \text { Carbon } \\
\text { County, Wyo., Sundance Formation, Redwater Shale Member, from a-loose block } \\
10.3 \mathrm{~m} \text { above base of member. }\end{array}$ \\
\hline 45 & 28402 & FC-\#1 & $\begin{array}{l}\text { G. N. Pipiringos, } 1961 \text {. Freezeout Mountains, southwest in NE } 1 / 4 \text { sec. } 26, T .24 \text { N., } \\
\text { R. } 80 \text { W., Carbon County, Wyo. Sundance Formation, } 22 \mathrm{~m} \text { above base of Redwater }\end{array}$ \\
\hline 45 & 28814 & $2-30 \mathrm{~W} F \mathrm{FC} \# 2$ & $\begin{array}{l}\text { G. N. Pipiringos, 1961. Freezeout Mountains, southwest, in sec. } 27, T \text { T. } 24 \text { N., R. } 80 \text { W., } \\
\text { about } 1.6 \mathrm{~km} \text { west of USGS Mesozoic loc. } 28402 \text {, Carbon County, Wyo. Sundance } \\
\text { Formation. Redwater Shale Member, float. }\end{array}$ \\
\hline 46 & 2382 & & $\begin{array}{l}\text { Charles Schuchert, 1899. East side of Freezeout Mountains around University of Wyo- } \\
\text { ming cabin in T. } 25 \mathrm{~N} \text {., R. } 78 \text { W., Carbon County, Wyo. Sundance Formation, Redwater } \\
\text { Shale Member from nodules above the belemnite beds. }\end{array}$ \\
\hline 46 & 2385 & ---------- & $\begin{array}{l}\text { Charles Schuchert, } 1899 \text {. Same place as USGS Mesozoic loc. } 2382 \text {. Fossils from belemnite } \\
\text { beds near middle of Sundance Formation. }\end{array}$ \\
\hline 46 & 30328 & I72-7-30B & $\begin{array}{l}\text { W. O. Ross, } 1972 \text {. Freezeout Mountains about } 1 \mathrm{~km} \text { south-southwest of T. B. Ranch houses } \\
\text { near center of } \mathrm{S}^{1 / 2} \mathrm{NE} 1 / 4 \text { sec. 7, T. } 24 \mathrm{~N} \text {., R. } 78 \mathrm{~W} \text {., T. B. quadrangle, Carbon County, Wyo } \\
\text { Sundance Formation, Redwater Shale Member, about } 9.7 \mathrm{~m} \text { below base of Windy Hill } \\
\text { Sandstone Member of Sundance. } \\
\text { R. W. Imlay and W. O. Ross, } 1972 \text {. Freezeout Mountains about } 0.8 \mathrm{~km} \text { southwest of T. B. } \\
\text { Ranch houses in } \mathrm{SW}^{1 / 4} \mathrm{SW} 1 / 4 \mathrm{SE}^{1 / 4} \text { sec. } 32, \mathrm{~T} \text {. } 25 \mathrm{~N} \text {., R. } 78 \mathrm{~W} \text {., T. B. Ranch quadrangle, } \\
\text { Carbon County, Wyo. Sundance Formation, } 4 \mathrm{~m} \text { above base of Redwater Shale Member } \\
\text { and } 0.6 \mathrm{~m} \text { below top of basal siltstone. }\end{array}$ \\
\hline
\end{tabular}


TABLE 7.-Description of Jurassic (late Callovian and Oxfordian) ammonite localities in the Western Interior regionof the United States-Continued

\begin{tabular}{|c|c|c|c|}
\hline $\begin{array}{l}\text { Loc. No. } \\
\text { (fig. 1) }\end{array}$ & $\begin{array}{l}\text { USGS Mesozoic } \\
\text { loc. No. }\end{array}$ & $\begin{array}{l}\text { Collector's } \\
\text { field No. }\end{array}$ & Collector, year of collection, description of locality, and stratigraphic assignment \\
\hline 46 & 30336 & I72-8-3D & $\begin{array}{l}\text { W. O. Ross, 1972. Freezeout Mountains in NW } 1 / 4 \mathrm{SE}^{1 / 4} \text { sec. } 32, \mathrm{~T} .25 \mathrm{~N} ., \mathrm{R} .78 \mathrm{~W} \text {., T. B. } \\
\text { Ranch quadrangle, Carbon County, Wyo. Sundance Formation, Redwater Shale Mem- } \\
\text { ber, } 6.7-8.5 \mathrm{~m} \text { above base. }\end{array}$ \\
\hline 47 & 31383 & I77-6-14A & $\begin{array}{l}\text { R. W. Imlay and G. N. Pipiringos, 1977. Flat Top anticline, west, in NW } 1 / 4 \mathrm{SE}^{1 / 4} \mathrm{NW} 1 / 4 \mathrm{sec} \text {. } \\
\text { 14, T. } 23 \text { N., R. } 79 \text { W., Como East quadrangle, Carbon County, Wyo. Sundance For- } \\
\text { mation, Redwater Shale Member, upper } 1.2 \text { m of lower siltstone unit. }\end{array}$ \\
\hline 47 & 31385 & I77-6-14C & $\begin{array}{l}\text { G. N. Pipiringos and R. W. Imlay, 1977. At same butte as type section of Pine Butte } \\
\text { Member in SE } 1 / 4 \mathrm{NW}^{1 / 4} \mathrm{NW} 1 / 4 \text { sec. } 14 \text {, T. } 23 \mathrm{~N} \text {., R. } 79 \mathrm{~W} \text {., Como East quadrangle, Carbon } \\
\text { County, Wyo. Sundance Formation, Redwater Shale Member, } 6 \text { m above base of lower } \\
\text { shale unit. }\end{array}$ \\
\hline 47 & 31387 & I77-6-14E & $\begin{array}{l}\text { R. W. Imlay, 1977. Flat Top anticline in NE } 1 / 4 \mathrm{NW}^{1 / 4} \mathrm{NW}^{1 / 2} \text { sec. } 14, \mathrm{~T} .23 \mathrm{~N} ., \mathrm{R} .79 \mathrm{~W} \text {., Como } \\
\text { East quadrangle, Carbon County, Wyo. Sundance Formation, } 22.5 \mathrm{~m} \text { above base of } \\
\text { Redwater Shale Member and } 2.4 \mathrm{~m} \text { below top of upper shale unit of Redwater Shale } \\
\text { Member. Equals bed } 33 \text { of that member as described by Pipiringos, (1957, p. } 46 \text { ). }\end{array}$ \\
\hline 47 & 9357 & 225 & $\begin{array}{l}\text { C. J. Hares, H. M. Robinson, and C. F. Bowen, } 1915 \text {. SW } 1 / 4 \text { sec. } 13 \text {, T. } 23 \text { N., R. } 79 \text { W., } \\
\text { Saddleback Hills quadrangle, Wyo. Sundance Formation, Redwater Shale Member. }\end{array}$ \\
\hline 48 & 29696 & 507 & $\begin{array}{l}\text { G. N. Pipiringos, } 1947 \text {. Como Bluff in SW } 1 / 4 \text { NE } 1 / 4 \text { sec. } 18, T .22 \mathrm{~N} \text {., R. } 77 \text { W., Carbon } \\
\text { County, Wyo. Sundance Formation, Redwater Shale Member, near top of bed } 28 \\
\text { described by Pipiringos }(1957 \text { p } 50 \text { ) }\end{array}$ \\
\hline 48 & 529 & -------- & I. C. Russell and A. Hyatt, 1888. Como Bluff area near Aurora in T. 22 N., R. 77 W., Carbon \\
\hline 48 & 2388 & ------ & $\begin{array}{l}\text { Charles Schuchert, } 1889 \text {. Como Bluff near station at Aurora, Wyo. Sundance Formation, } \\
\text { Redwater Shale Member. }\end{array}$ \\
\hline $\begin{array}{l}48 \\
49\end{array}$ & $\begin{array}{l}11177 \\
28816\end{array}$ & 127 & $\begin{array}{l}\text { W. T. Lee, 1922. Como Ridge, Wyo. Sundance Formation, Redwater Shale Member. } \\
\text { G. N. Pipiringos, } 1963 \text {. Isolated outcrop in SE } 1 / 4 \mathrm{NW}^{1 / 4} \mathrm{NW} 1 / 4 \text { sec. } 20, \mathrm{~T} \text {. } 28 \mathrm{~N} \text {., R. } 77 \mathrm{~W} \text {., } \\
\text { Squaw Spring quadrangle, } 56 \mathrm{~km} \text { S. } 18^{\circ} \mathrm{E} \text {. of Casper, Carbon County, Wyo. Sundance } \\
\text { Formation. Redwater Shale Member, basal bed. }\end{array}$ \\
\hline 50 & 20519 & F20 & J. D. Love, 1946. Chalk Butte area, SW 1/4 sec. 9, T. 31 N., R. 71 W., Converse County, Wyo. \\
\hline 50 & 20520 & ------.... & J. D. Love, 1946. Same location as Mesozoic loc. 20519. Sundance Formation, float from \\
\hline 50 & 30312 & I72-8-17B & $\begin{array}{l}\text { W. O. Ross and R. W. Imlay, } 1972 . \mathrm{NW}^{1 / 4} \mathrm{NW}^{1 / 4} \text { sec. } 9 \text {, T. } 31 \mathrm{~N} \text {., R. } 71 \mathrm{~W} \text {., Chalk Butte } \\
\text { quadrangle, Converse County, Wyo. Sundance Formation, Redwater Shale Member, in } \\
\text { shale } 8-10 \mathrm{~m} \text { above base of member. }\end{array}$ \\
\hline 51 & 28815 & $\begin{array}{l}3-5 \mathrm{NW} F C \\
\quad \# 2\end{array}$ & 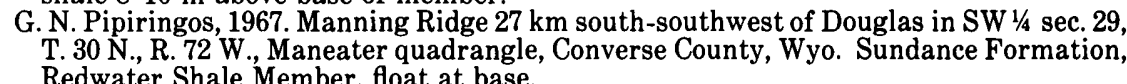 \\
\hline 51 & 30327 & I72-8-16A & $\begin{array}{l}\text { R. W. Imlay, } 1972 \text {. Manning Ridge in SE } 1 / 4 \mathrm{NW}^{1 / 4} \mathrm{SW} 1 / 4 \text { sec. } 29, \mathrm{~T} .30 \mathrm{~N} ., \mathrm{R} .72 \mathrm{~W} \text {., } \\
\text { Maneater quadrangle, Converse county, Wyo. Sundance Formation, Redwater Shale }\end{array}$ \\
\hline 52 & 20529 & SD-9 & N. M. Denson, 1946. Glendo area in SE $1 / 4 \mathrm{SW}^{1 / 4}$ sec. $36, T .31$ N., R. 67 W., Niobrara County, \\
\hline 53 & 20531 & D-15 & $\begin{array}{l}\text { N.M. Denson, } 1946 \text {. Glendo area, in NE } 1 / 4 \mathrm{SW}^{1 / 4} \text { sec. } 13, \mathrm{~T} .29 \text { N., R. } 68 \text { W., Platte County, } \\
\text { Wyo. Sundance Formation, Redwater Shale Member. }\end{array}$ \\
\hline 54 & 29528 & I66-76D & $\begin{array}{l}\text { W. O. Ross, 1966. Black Hills area in NW 1/4 } \mathrm{SW}^{1 / 1} / \mathrm{SW}^{1 / 4} \mathrm{sec} .28 \text {, T. } 56 \mathrm{~N} ., \mathrm{R} .63 \mathrm{~W} \text {., Mona } \\
\text { quadrangle, Crook County Wyo. Sundance Formation, Redwater Shale Member, con- } \\
\text { cretionary gray shale. }\end{array}$ \\
\hline 54 & 29532 & I66-7-6F & $\begin{array}{l}\text { W. O. Ross, Mario Suarez, and R. W. Imlay, 1966. Black Hills area about } 5 \mathrm{~km} \text { west of } \\
\text { Mona in } \mathrm{SW}^{1 / 4} \mathrm{NW}^{1 / 4} \mathrm{SW} 1 / 4 \text { sec. } 28 \text {, T. } 56 \text { N., R. } 63 \mathrm{~W} \text {., Mona quadrangle, Crook County, } \\
\text { Wyo. Sundance Formation, Redwater Shale Member, clay shale exposed on east side } \\
\text { of road. }\end{array}$ \\
\hline 55 & 16615 & ------ & $\begin{array}{l}\text { L. W. Stillwell, } 1902 \text {. Black Hills area, } 6.4 \mathrm{~km} \text { west of Devils Tower in sec. } 10 \text {, T. } 53 \mathrm{~N} \text {., } \\
\text { R. } 66 \text { W. Crook County. Wyo. Sundance Formation, Redwater Shale Member. }\end{array}$ \\
\hline 56 & 31717 & & $\begin{array}{l}\text { Collector unknown. Black Hills area, in foothills west of Bear Lodge Mountains, Crook } \\
\text { County, Wyo. Probably in sec. 15, T. } 53 \text { N., R. } 63 \text { W. Sundance Formation, Redwater }\end{array}$ \\
\hline 57 & 30323 & $\mathrm{I} 72-8-10 \mathrm{~B}$ & $\begin{array}{l}\text { W. O. Cobban, R. W. Imlay., W. O. Ross, John Callomon, and Tove Birkelund, } 1972 . \\
0.8 \text { km south of Aladdin in SE } 1 / 1 / 4 \mathrm{~N}^{1 / 4} \text { sec. } 32 \text {, T. } 54 \mathrm{~N} . \text {, R. } 61 \text { W., Crook County, Wyo. } \\
\text { Sundance Formation, Redwater Shale Member. From a concretionary bed in shale } \\
14 \mathrm{~m} \text { above top of the Lak Member of Sundance. }\end{array}$ \\
\hline 57 & 30324 & I72-8-10C & $\begin{array}{l}\text { Cobban and others, } 1972 \text {. Same locality data as USGS Mesozoic loc. 30323. From a } \\
\text { concretionary bed in shale } 15 \mathrm{~m} \text { above top of the Lak Member of the Sundance For- } \\
\text { mation, and } 22 \mathrm{~m} \text { below top of measured section. }\end{array}$ \\
\hline 57 & 30325 & I72-8-10D & $\begin{array}{l}\text { Cobban and others, 1972. Same locality data as USGS Mesozoic loc. } 30323 \text {. From a } \\
\text { concretionary bed in shale } 20.4 \mathrm{~m} \text { below exposed top of measured section. }\end{array}$ \\
\hline 57 & 30326 & I72-8-10E & Cobban and others, 1972. Same locality data as USGS Mesozoic loc. 30323. From shale \\
\hline 57 & 31719 & & $\begin{array}{l}\text { Collector unknown. } 5 \mathrm{~km} \text { southwest of Barrett (Aladdin), Crook County, Wyo. Probably } \\
\text { in NE } 1 / 4 \text { sec. } 1 \mathrm{~T} .53 \mathrm{~N} \text {. R. } 62 \mathrm{~W} \text {. Sundance Formation, Redwater Shale Member. }\end{array}$ \\
\hline 58 & 16616 & & $\begin{array}{l}\text { L. W. Stillwell, } 1902.2 .2 \mathrm{~km} \text { north of Redwater Creek in Wyoming near State line with } \\
\text { South Dakota. Probably in sec. } 7 \text {, T. } 53 \text { N., R. } 60 \text { W., in Crook County. Sundance } \\
\text { Formation, Redwater Shale Member. }\end{array}$ \\
\hline 58 & 29523 & I66-7-3D & $\begin{array}{l}\text { Mario Suarez, 1966. About } 5 \mathrm{~km} \text { north of Beulah in west-central part of sec. } 19, \mathrm{~T} .53 \mathrm{~N} \text {., } \\
\text { R. } 60 \mathrm{~W} \text {., Crook County, Wyo. Sundance Formation, Redwater Shale Member, above } \\
7.6 \mathrm{~m} \text { of siltstone. }\end{array}$ \\
\hline 58 & 29541 & $166-7-3 \mathrm{C}$ & $\begin{array}{l}\text { R. W. Imlay, 1966. Same general location as USGS Mesozoic loc. 29523. At head of } \\
\text { westward-draining gulch about } 3.2 \mathrm{~km} \text { north-northwest of Beulah. Sundance For- } \\
\text { mation, Redwater Shale Member, at top of } 7.6 \mathrm{~m} \text { of siltstone. }\end{array}$ \\
\hline
\end{tabular}


TABLE 7.-Description of Jurassic (lateCallovian and Oxfordian) ammonitelocalities in the Western Interior region of the United States-Continued

\begin{tabular}{|c|c|c|c|}
\hline $\begin{array}{l}\text { Loc. No. } \\
\text { (fig. 1) }\end{array}$ & $\begin{array}{l}\text { USGS Mesozoic } \\
\text { loc. No. }\end{array}$ & $\begin{array}{l}\text { Collector's } \\
\text { field No. }\end{array}$ & Collector, year of collection, description of locality, and stratigraphic assignment \\
\hline 59 & 31716 & -------- & $\begin{array}{l}\text { Collector unknown. Two miles south of Belle Fourche River, near Bear Lodge Butte } \\
\text { (Devils Tower), Crook County, Wyo. Possibly represented by exposures at SE } 1 / 4 \text { sec. } 3 \text {, } \\
\text { T. } 53 \text { N., R. 63 W. Sundance Formation, Redwater Shale Member. }\end{array}$ \\
\hline 60 & 19382 & -..-- & $\begin{array}{l}\text { Collector unknown. } 0.8 \mathrm{~km} \text { northeast of the Johnson Horse Ranch near the Belle Fourche } \\
\text { River. Probably near the center of T. } 52 \text { N., R. } 65 \text { W., Crook County, Wyo. }\end{array}$ \\
\hline 61 & 29521 & I66-7-6D & $\begin{array}{l}\text { Mario Suarez, 1966. On east side of road in SW } 1 / 4 \mathrm{SE}^{1 / 4} \mathrm{NW}^{1 / 4} \text { sec. } 25 \text {, T. } 52 \mathrm{~N} ., \mathrm{R} .66 \mathrm{~W} \text {., } \\
\text { Nefsy Divide quadrangle, Crook County, Wyo. Sundance Formation, Redwater Shale } \\
\text { Member. }\end{array}$ \\
\hline 61 & 29529 & I66-7-5F & $\begin{array}{l}\text { W. O. Ross and R. W. Imlay, 1966. On west side of Belle Fourche road near center of NW } 1 / 4 \\
\text { sec. } 25 \text {, T. } 52 \text { N., R. } 66 \text { W., Nefsy Divide quadrangle, Crook County, Wyo. Sundance } \\
\text { Formation, Redwater Shale Member. }\end{array}$ \\
\hline 61 & 29535 & I66-7-6C & $\begin{array}{l}\text { Mario Suarez, 1966. About } 15 \mathrm{~m} \text { east of road in NW } 1 / 4 \mathrm{NE}^{1 / 4} \mathrm{SW} 1 / 4 \text { sec. } 25, \mathrm{~T} .52 \mathrm{~N} \text {., } \\
\text { R. } 66 \text { W., Nefsy Divide quadrangle, Crook County, Wyo. Sundance Formation, Red- } \\
\text { water Shale Member. }\end{array}$ \\
\hline 61 & 29540 & I66-7-6B & $\begin{array}{l}\text { R. W. Imlay, } 1966 \text {. About } 122 \mathrm{~m} \text { east of road in } \mathrm{SE}^{1 / 4} \mathrm{NE}^{1 / 4} \mathrm{SW} 1 / 4 \text { sec. } 25, \mathrm{~T} \text {. } 52 \mathrm{~N} \text {., } \\
\text { R. } 66 \text { W., Nefsy Divide quadrangle Crook County, Wyo. Sundance Formation, Red- } \\
\text { water Shale Member, } 23-30 \mathrm{~m} \text { below top. }\end{array}$ \\
\hline 62 & 31718 & - & $\begin{array}{l}\text { Collector unknown. "Aladdin Stage road at south fork of Redwater Creek, Crook County, } \\
\text { Wyo." Probably represented by outcrops in the southern part of sec. 7, T. } 52 \text { N., } \\
\text { R. } 61 \text { W. Sundance Formations, Redwater Shale Member. }\end{array}$ \\
\hline 63 & 16620 & --------- & $\begin{array}{l}\text { L. W. Stillwell, } 1902.6 .4 \mathrm{~km} \text { west of Sundance, Crook County, Wyo. Sundance Formation, } \\
\text { Redwater Shale Member. }\end{array}$ \\
\hline 63 & 29534 & I66-7-5D & $\begin{array}{l}\text { R. W. Imlay, 1966. West of Sundance in SW } 1 / 4 \text { sec. 17, T. } 51 \text { N., R. } 63 \mathrm{~W} \text {., Crook County, } \\
\text { Wyo. Sundance Formation, Redwater Shale Member about } 17.6 \mathrm{~m} \text { below top and } 9 \mathrm{~m} \\
\text { below an oyster bed. }\end{array}$ \\
\hline 63 & 29531 & I $66-7-5 \mathrm{C}$ & $\begin{array}{l}\text { R. W. Imlay and Mario Suarez, 1966. Black Hills area. Northeast side of road in SE } 1 / 4 \\
\text { SW 1/4 sec. 17, T. } 51 \text { N., R. } 63 \text { W., Crook County, Wyo. Sundance Formation, Redwater } \\
\text { Shale Member, about } 21 \mathrm{~m} \text { below top and } 12 \mathrm{~m} \text { below an oyster bed. }\end{array}$ \\
\hline 64 & 30114 & I71-6-27A & $\begin{array}{l}\text { R. W. Imlay, 1971. Black Hills area in NE } 1 / 4 \mathrm{NE}^{1 / 4} \mathrm{NW}^{1 / 4} \mathrm{sec} .1, \mathrm{~T} .48 \mathrm{~N} ., \mathrm{R} .63 \mathrm{~W} \text {., Weston } \\
\text { County Wyo. Sundance Formation, Redwater Shale Member, near middle of basal } \\
\text { siltstone unit. }\end{array}$ \\
\hline 65 & 30108 & $171-6-25 \mathrm{~A}$ & $\begin{array}{l}\text { R. W. Imlay and W. O. Ross, 1971. About } 91.5 \mathrm{~m} \text { south of section measured by Imlay ( } 1947 \text {, } \\
\text { p. } 271 \text { ) in NW } 1 / 4 \mathrm{NE} 1 / 4 \text { sec. } 18, \mathrm{~T} \text {. } 45 \mathrm{~N} ., \mathrm{R} .60 \mathrm{~W} \text {., Weston County, Wyo. Sundance } \\
\text { Formation, Redwater Shale Member, } 15-18 \mathrm{~m} \text { above base, from slabs of coquina in } \\
\text { siltstone. }\end{array}$ \\
\hline 65 & 30109 & I17-6-25B & $\begin{array}{l}\text { R. W. Imlay and W. O. Ross, 1971. West side of Stockade Beaver Creek in east-central part } \\
\text { of sec. } 18 \text {, T. } 45 \text { N., R. } 60 \text { W., Weston County, Wyo. Sundance Formation, Redwater } \\
\text { Shale Member. }\end{array}$ \\
\hline 65 & 30110 & I71-6-25C & $\begin{array}{l}\text { R. W. Imlay, } 1971 \text {. About } 124 \mathrm{~m} \text { south of USGS Mesozoic loc. } 30109 \text { and slightly east of } \\
\text { center of sec. } 18 \text {, T. } 45 \mathrm{~N} \text {., R. } 60 \text { W., Weston County, Wyo. Sundance Formation, }\end{array}$ \\
\hline 66 & 19570 & I45-6-23A & $\begin{array}{l}\text { R. W. Imlay and W. D. Saalfrank, } 1945 \text {. About } 1.6 \mathrm{~km} \text { east of road to Beulah, } 1.6 \mathrm{~km} \text { north } \\
\text { of Redwater Creek, and } 16 \mathrm{~km} \text { northwest of Spearfish in SE } 1 / 4 \mathrm{sec} .4, \mathrm{~T} \text {. } 7 \mathrm{~N} \text {., R. } 1 \mathrm{E} \text {., } \\
\text { Butte County, S. Dak. Sundance Formation, Redwater Shale Member, } 16.7 \mathrm{~m} \text { above } \\
\text { base of unit } 22 \text { of published measured section (Imlay, 1947, p. 267) }\end{array}$ \\
\hline 66 & 27640 & & $\begin{array}{l}\text { N. F. Sohl, } 1960.1 .6 \mathrm{~km} \text { north of Redwater Creek in SE } 1 / 4 \text { sec. } 4, \mathrm{~T}, 7 \mathrm{~N} \text {., R. } 1 \mathrm{E} \text {., Butte } \\
\text { County, S. Dak. Sundance Formation, Redwater Shale Member, } 9 \mathrm{~m} \text { above base of unit } \\
22 \text { of published measured section (Imlay, } 1947, \text { p. } 266,267 \text { ). }\end{array}$ \\
\hline 66 & 29524 & I66-6-26B & $\begin{array}{l}\text { R. W. Imlay, } 1966 \text {. } 1.6 \mathrm{~km} \text { north of Redwater Creek in NE } 1 / 4 \mathrm{SW}^{1 / 4} \mathrm{SE}^{1 / 4} \mathrm{sec} .4, \mathrm{~T} .7 \mathrm{~N} \text {., } \\
\text { R. } 1 \text { E., Butte County, S. Dak. Sundance Formation, Redwater Shale Member } 14.3 \mathrm{~m} \\
\text { above Lak Member of Sundance and } 4.2 \mathrm{~m} \text { above base of clay shale that overlies } 7.3 \mathrm{~m}\end{array}$ \\
\hline 67 & 30322 & I72-8-10S & $\begin{array}{l}\text { W. A. Cobban, 1972. About } 1.6 \mathrm{~km} \text { northeast of Spearfish in NE1/4 SW } 1 / 4 \mathrm{SE}^{1 / 4} \mathrm{sec} .3 \text {, } \\
\text { T. } 6 \mathrm{~N} \text {, R. } 2 \text { E., Jolly quadrangle, Lawrence County, S. Dak. Sundance Formation, float } \\
\text { on Redwater Shale Member. }\end{array}$ \\
\hline 68 & 10635 & 1859 & $\begin{array}{l}\text { T. W. Stanton, } 1921 \text {. On north side of Spring Creek east of road from Rapid City to } \\
\text { Rockerville. Probably in E1/2 sec. } 3 \text {, T. } 1 \text { S., R. } 7 \text { E., Pennington County, S. Dak. } \\
\text { Sundance Formation. Redwater Shale Member. }\end{array}$ \\
\hline 69 & 30107 & I71-6-23B & $\begin{array}{l}\text { W. O. Ross, } 1971 \text {. Head of Dugout Creek in west-central part of SE } 1 / 4 \mathrm{SW} 1 / 4 \mathrm{sec} .19, \mathrm{~T} .4 \mathrm{~S} \text {., } \\
\text { R. } 1 \text { E. Custer County. S. Dak. Sundance Formation. Redwater Shale Member, float }\end{array}$ \\
\hline 70 & 30115 & I71-6-28A & $\begin{array}{l}\text { W. O. Ross and R. W. Imlay, } 1971 \text {. On west bank of a gulch in NW } 1 / 4 \mathrm{NE} 1 / 4 \mathrm{SW} 1 / 4 \mathrm{sec} \text {. } 28 \text {, } \\
\text { T. } 6 \mathrm{~S} \text {., R. } 2 \mathrm{E} \text {., Custer County, S. Dak. Sundance Formation, Redwater Shale Member, } \\
\text { from base of } 15-18 \mathrm{~m} \text { of grayish-black siltstone overlain by } 3.6 \mathrm{~m} \text { of glauconitic sand- } \\
\text { stone that directly underlies Morrison Formation. }\end{array}$ \\
\hline 71 & 30105 & I71-6-28B & $\begin{array}{l}\text { R. W. Imlay and W. O. Ross, } 1971 \text {. From gully in NE. cor. sec. 14, T. } 7 \text { S., R. } 2 \text { E., Fall River } \\
\text { County, S. Dak. Sundance Formation, Redwater Shale Member, about } 12 \mathrm{~m} \text { below } \\
3-4.5 \mathrm{~m} \text { of sandstone at the top of the member. }\end{array}$ \\
\hline 72 & 25176 & F5 & $\begin{array}{l}\text { R. D. Smith and V. R. Wilmarth, } 1954 \text {. On Chord's Road south of Matias Peak in SE } 1 / 4 \text { sec. } \\
\text { 34, T. } 7 \text { S., R. } 3 \text { E., Minnekahta quadrangle, Fall River County, S. Dak. Sundance } \\
\text { Formation, Redwater Shale Member. }\end{array}$ \\
\hline 73 & 19567 & I45-6-16D & $\begin{array}{l}\text { R. W. Imlay and W. G. Saalfrank, } 1945 \text {. About } 3.2 \mathrm{~km} \text { east-southeast of Minnekahta in } \\
\text { SE } 1 / 4 \mathrm{SW} 1 / 4 \mathrm{sec} \text {. } 21 \text {, T. } 7 \mathrm{~S} \text {., R. } 4 \mathrm{E} \text {., Fall River County, S. Dak. Sundance Formation, } \\
\text { Redwater Shale Member, float } 0.61 \mathrm{~m} \text { above base of unit } 12 \text { of Imlav (1947. }\end{array}$ \\
\hline 73 & 19568 & $\mathrm{I} 45-6-16 \mathrm{E}$ & $\begin{array}{l}\text { R. W. Imlay and W. G. Saalfrank, } 1945 \text {. Same location as USGS Mesozoic loc. } 19567 . \\
\text { Sundance Formation, float about } 4 \mathrm{~m} \text { above base of Redwater Shale Member. (See } \\
\text { Imlay, 1947, p. 269, unit 12.) }\end{array}$ \\
\hline 73 & 19571 & I45-6-16C & $\begin{array}{l}\text { R. W. Imlay and W. G. Saalfrank, 1945. Same location as USGS Mesozoic loc. } 19567 . \\
\text { Sundance Formation, Redwater Shale Member, } 10.6 \mathrm{~m} \text { above base of a soft fissile gray } \\
\text { shale constituting unit } 12 \text { of Imlay }(1947, \text { p. } 269)\end{array}$ \\
\hline
\end{tabular}


TABLE 7.-Description of Jurassic (late Callovian and Oxfordian) ammonitelocalities in the Western Interior region of the United States_Continued

\begin{tabular}{c|c|c|c}
\hline $\begin{array}{c}\text { Loc. No. } \\
\text { (fig. 1) }\end{array}$ & $\begin{array}{c}\text { USGS Mesozoic } \\
\text { loc. No. }\end{array}$ & $\begin{array}{c}\text { Collector's } \\
\text { field No. }\end{array}$ & Collector, year of collection, description of locality, and stratigraphic assignment \\
\hline 73 & 29538 & I66-6-23A & $\begin{array}{c}\text { R. W. Imlay, W. O. Ross and G. N. Pipiringos, 1966. In SW. cor. sec. 21, T. 7 S., R. 4 E., } \\
\text { Fall River County, S. Dak. Sundance Formation, Redwater Shale Member, from near } \\
\text { base of soft claystone unit overlying siltstone. } \\
\text { R. W. Imlay and W. O. Ross, 1966. About 8 km south-southwest of Hot Springs in NE. cor. } \\
\text { sec. 15, T. 8 S., R. 5 E., Fall River County, S. Dak. Sundance Formation, Redwater Shale } \\
\text { Member. In clay shale 11.5 m above base of member and 4.5 m above top of basal } \\
\text { siltstone unit. }\end{array}$ \\
\hline
\end{tabular}

\section{SYSTEMATIC DESCRIPTIONS \\ Family CARDIOCERATIDAE Siemiradzki, 1891 \\ Subfamily CARDIOCERATINAE Siemiradzki, 1891 \\ Genus QUENSTEDTOCERAS Hyatt, 1877 \\ Subgenus LAMBERTICERAS Buckman, 1920}

Quenstedtoceras (Lamberticeras) collieri Reeside

Plate 1, figures 1-12

Quenstedtoceras collieri Reeside, 1919, U.S. Geol. Survey Prof. Paper 118 , p. 14, pl. 1, figs. 1-18.

Quenstedtoceras (Bourkelamberticeras) collieri Reeside, Imlay, 1948,

U.S. Geol. Survey Prof. Paper 214-B, p. 25, pl. 7, figs. 12, 14, 15, 18.

This species is represented by several hundred septate specimens from mountains in north-central Montana.

It has a compressed shell, a very narrowly rounded venter, a whorl section that changes during growth from ovate to subtriangular, a fairly narrow umbilicus, and a vertical umbilical wall that rounds evenly into the flanks on small septate specimens and abruptly on the large septate specimens. Its living chamber is unknown.

The innermost whorls are smooth (pl. 1, figs. 2,3 ) to diameters of about $8 \mathrm{~mm}$. At diameters of 8 to $18 \mathrm{~mm}$, the whorls bear fairly sharp ribs that trend backward on the umbilical wall, incline slightly forward on the lower part of the flanks, curve forward more sharply on the upper part of the flanks, and then form pronounced chevrons on the venter. About half the ribs bifurcate at or a little below the middle of the flanks. A few ribs arise freely at about the same position. All ribs are strongest on the uppermost part of the flanks and weakest on the middle part of the flanks.

Adorally, at diameters greater than $18 \mathrm{~mm}$, the ribs gradually become higher and sharper near the umbilicus, more prominent on and near the venter, weak to faint at and a little below the middle of the flanks, and indistinctly united at furcation points a little below the middle of the flanks.

This species differs from $Q$. (L.) lamberti (J. Sowerby) (Arkell and others, 1957, p. L303, fig. 372-1a, b; Buckman, 1920, v. 3, pl. 154 and p. 14) by having fewer intercalated ribs. It differs from $Q$. (L.) henrici $\mathrm{R}$. Douville (1912, p. 55, pl. 4, figs. 24-32) by having a higher and thinner whorl section and weaker ribbing near the middle of the flanks. These characteristics likewise distinguish $Q$. (L.) collieri Reeside from the species of $Q$. (Lamberticeras) that are present in the upper Callovian beds of north-central British Columbia (Frebold and Tipper, 1975, p. 148, 149, pl. 1, figs. 1-11).

Quenstedtoceras (Lamberticeras) collieri Reeside has been found only in the basal beds of the Swift Formation exposed in the Little Rocky Mountains and the Bearpaw Mountains in north-central Montana. In those mountains, the species has been collected at 16 localities as highly compressed specimens in splintery shale in the lower 12 to $15 \mathrm{~m}$ of the formation. The species has also been collected at 13 localities as loose pyritized nodules on the weathered surfaces overlying the lower part of that shale. Other ammonities have not been found with $Q$. (L.) collieri in the layers of splintery shale. The species has been found, however, with other pyritized ammonites at five localities on the weathered surfaces of the shale. Such associations include Quenstedtoceras (Pavloviceras) cf. Q. (P.) omphaloides (J. Sowerby) at USGS Mesozoic loc. 19204, 19577-19580; Cardioceras (Scarburgiceras) americanum Reeside at USGS Mesozoic loc. 19204; C. (Maltoniceras) sundancense Reeside at USGS Mesozoic loc. 19577; C. (Scoticardioceras) cf. $C$. (S.) stillwelli Reeside at USGS Mesozoic loc. 19579; Prososphinctes? sp. at USGS Mesozoic loc. 19579; and Grossouvria? sp. at USGS Mesozoic loc. 19577.

Types.-Holotype, USNM 32298; paratypes, USNM 32299-32301; hypotypes, USNM 104137, 303601-303606, and 303724 .

Occurrences.-Swift Formation, lower part, in the Little Rocky Mountains, north-central Montana at USGS Mesozoic locs. 9832, 9835, 18728, 18761, 19204, 19210, 19577-19580, 19582, 29288-29290, 30083, 30084, 30086, and 30101; in the Bearpaw Mountains, north-central Montana at USGS Mesozoic locs. 19199, 27006, 2927729279, 30089, 30090, and 30093-30099.

\section{Subgenus Pavioviceras Buckman, 1920}

\section{Quenstedtoceras (Pavloviceras) cf. Q. (P.) omphaloides (J. Sowerby)}

Plate 2, figures 1-8

cf. Pavloviceras omphaloides J. Sowerby. Buckman, 1921, Type ammonites, v. 3, p. 18, pl. 195

This species is represented by 20 fragmentary and mostly poorly preserved pyritized septate specimens. On the best preserved specimens, the whorls are stout, depressed, subovate, a little wider than high, become 
wider during growth, and do not bear a keel or even a slightly sharpened venter. The umbilicus is fairly wide.

The ribs are sharp, wiry, and are strongest on the venter. On the smaller specimens, the ribs trend forward on the upper two-thirds of the flanks and then cross the venter nearly transversely. On the largest specimens, the ribs trend nearly radially on the lower third of the flanks, trend only slightly backward on the upper two-thirds of the flanks, and then arch forward gently on the venter. One-third to one-half of the primary ribs bifurcate at about one-third the height of the flanks. In each rib pair, the posterior rib curves backward more strongly than does the anterior rib.

The largest specimen shows a great resemblance to $Q$. (Pavloviceras) omphaloides (J. Sowerby) as figured by Buckman (1921, v. 3, p. 18, pl. 195) and differs mainly by having a rounder whorl section and a little less flexuous ribbing. Its ribbing is even more similar to that on $Q$. $(P$.) bathyomphalum Buckman as figured by Maire (1938, pl. 5, figs. 9, 9a, 10, 10a), but this largest specimen apparently has a wider umbilicus and a more rounded whorl section. It likewise resembles the holotype of $Q$. $(P$. ) schaunburgensis Maubeuge (1975, p. 175, fig. G1982 on p. 176) but differs by having less flexuous ribbing.

Figured specimens.-Swift Formation, 4.5 to $14 \mathrm{~m}$ above its base, in the Little Rocky Mountains, Mont., at USGS Mesozoic locs. 19204, 19577-19580.

\section{Quenstedtoceras (Pavloviceras) latum (Reeside)}

Plate 1, figures 13, 15-20, 22-24, 29

Cardioceras? latum Reeside, 1919, U.S. Geol. Survey Prof. Paper 118, p. 37, pl. 20, figs. 7-12 (probably not figs. 13-16).

This species, represented by seven specimens, was based originally on six very small septate specimens and one much larger nonseptate fragment of an outer whorl. That larger fragment differs from the small specimens by having very strong, widely spaced primary ribs and a nearly smooth obtusely fastigate venter. The lack of ribbing on the venter of the larger fragment indicates that this fragment probably does not belong to the subgenus Pavloviceras, which retains strong ribbing on its adult whorls. Furthermore, an ammonite of moderate size has now been found whose inner whorls (pl. 1, figs. 22-24) bear ribbing similar to that on the small specimens of $Q$. ( $P$.) latum that were illustrated by Reeside and whose outer whorl is likewise fairly similar (see pl. 1, figs. 13, 29).

The small specimens, which include the holotype, have a fairly wide umbilicus; depressed whorls that become more depressed during growth; sharp primary ribs that curve forward to the middle of the flanks where they are strongest; somewhat weaker secondary ribs that arise mostly in pairs from the primary ribs, curve backward on the upper parts of the flanks, and then recurve forward gently to the middle of the venter where they form shallow chevrons. On some specimens, these chevrons are much less shallow than they are on other specimens.
The innermost exposed whorl of the larger ammonite (pl. 1, figs. 22-24) bears ribbing that appears to be a little sharper than that on the small type specimens but is comparable in sharpness with the ribbing on some specimens that Reeside included in the species (USGS Mesozoic loc. 2388 and 16616). The main difference is in the presence of an obtusely fastigate venter and somewhat sharper ribs on the venter.

On the nextouter whorl, of which one-fourth represents body chamber, the primary ribs remain sharp and gradually become more widely spaced and higher. The secondary ribs are much weaker than the primary ribs, outnumber them about three to one, and do not fade on the venter. The rib pattern is identical with that on the inner whorl and closely similar to that on the type specimens.

Type.-Holotype, USNM 32354; paratypes, USNM 32353 and USNM 32355 (probably invalid as discussed herein); hypotypes, USNM 303611-303613.

Compared figured specimen.-USNM 303614 (pl. 1, fig. 14, 21).

Occurrences.-Redwater Shale Member of the Sundance Formation in Wyoming at USGS Mesozoic locs. 2388, 16615, 16616, 19382, and 30341. Possibly represented by small pyritized specimens (pl. 1, figs. 14,21) in basal part of the Swift Formation in the Little Rocky Mountains, Montana, at Mesozoic loc. 19204.

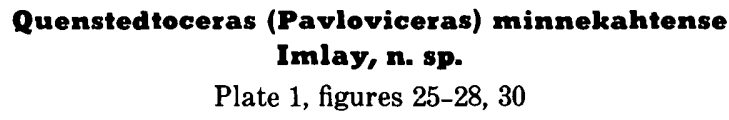

This species is represented by two septate specimens. Its whorl section is ovate, a little wider than high, and is widest at about one-fourth of the whorl height. Its umbilicus is fairly narrow. The umbilical wall is vertical and rounds evenly into the flanks. The venter is obtusely fastigate on the small specimen and on the adapical end of the large specimen.

Ribbing is strong. On the smaller specimen, the primary ribs trend radially, are fairly strong and moderately spaced, but became stronger and more widely spaced adorally and terminate at about the top of the lower third of the flanks. Weaker secondary ribs arise in pairs from the primary ribs, and generally one secondary rib arises freely between forked ribs near the middle of the flanks. The secondary ribs also trend nearly radially or recurve slightly backward on the upper part of the flanks, then curve forward on the venter, where they form marked chevrons.

On the larger specimen, the primary ribs adorally become much stronger, more widely spaced, and terminate rather abruptly at about the top of the lower third of the flanks. From them arise three to four low broad secondary ribs. Generally, one secondary rib arises freely between the forked ribs. As a result, the secondary ribs outnumber the primary ribs a little more than four to one. Adorally, the secondary ribs become very broad, and the spaces between them become very narrow. 
This species differs from $G$. (P.) latum Reeside by having a narrower umbilicus, a higher, rounder whorl section, much less flexuous ribbing, and apparently much stronger primary ribs. Its coarse primary ribs resemble those of $Q$. (P.) stibarum Buckman (1921, pl. 197), but it differs by having many more secondary ribs per primary rib.

Types.-Holotype, USNM 303615; paratype, USNM 303616.

Occurrences.-Redwater Shale Member of the Sundance Formation in Wyoming at USGS Mesozoic loc. 16616; in South Dakota, at USGS Mesozoic loc. 19571.

\section{Genus GOLIATHICERAS Buckman, 1919}

\section{Goliathiceras (Goliathiceras) suspectum (Reeside)}

Plate 2, figures 9, 10; plate 3, figures 1-7

Quenstedticeras? suspectum Reeside, 1919, U.S. Geol. Survey Prof.

Paper 118, p. 16, pl. 2, figs. 2-4, pl. 3, pl. 4, figs. 5-7, pl. 5, figs. 1-2.

This species, represented by 16 specimens, has a robust shell; a faintly pinched venter at small diameters; stout rounded whorls that are wider than high at diameters greater than $30 \mathrm{~mm}$; an umbilicus whose width is about 34 percent of the diameter; a high vertical umbilical wall that rounds rather abruptly into the flanks; and a scaphitoid body chamber.

On septate whorls of small and intermediate size, the primary ribs are high, rounded, trend radially, or incline slightly forward on the lower third of the flank, and then pass into pairs of slightly weaker secondary ribs. Most pairs of secondary ribs are separated by single ribs that arise freely near the middle of the flanks. All secondary ribs curve forward on the upper part of the flanks and form chevrons on the venter. Those chevrons are fairly angular on the small septate specimens but become less so on the larger septate specimens. Some specimens have somewhat coarser ribbing than do other specimens of comparable size, but all specimens become less coarsely ribbed adorally. The largest septate whorls bear weak broad ribs only on the venter and on the upper parts of the flanks. The largest adult body chamber is smooth except for striae.

This species is characterized by its stout rounded whorls, by an umbilicus that is intermediate between fairly wide and fairly narrow, and by regular ribbing persisting to a diameter of about $120 \mathrm{~mm}$ and then gradually fading out at a diameter of about $170 \mathrm{~mm}$.

The species differs from Quenstedtoceras (Goliathites) hoveyi (Reeside) by having broader whorls, a wider umbilicus, and much stronger and more persistent ribbing.

Types.-Holotype, USNM 32304; paratypes, USNM 32303 , 32305, 32359; hypotypes, USNM 303617-303619.

Occurrences.-Redwater Shale Member of the Sundance Formation in Wyoming at USGS Mesozoic locs. 529, 19382, 29535, 29696, 30110, and 30336; in South Dakota at Mesozoic loc. 10635.

\section{Subgenus GOLIATHITES Axkell, 1943 \\ Goliathiceras (Goliathites) hoveyi (Reeside)}

Plate 5, figures 1, 2; plate 6, figures 1-4

Ammonites (Aegoceras) subtumidum Whitfield and Hovey, 1906, Am. Mus. Nat. History Bull., v. 22, art. 23, p. 400, pl. 62.

Quenstedtoceras? hoveyi Reeside, n. sp., 1919, U.S. Geol. Survey Prof. Paper 118, p. 15, pl. 1, figs. 9-14, pl. 2, fig. 1.

This species is represented by seven medium to large specimens. It has a stout shell, a rounded venter, whorls that are wider than high, a narrow umbilicus, a vertical umbilical wall, and an abruptly rounded umbilical shoulder.

On medium-sized septate specimens that are at least $65 \mathrm{~mm}$ in diameter, the primary ribs are nearly straight, broadly rounded, and divide near the umbilicus into two or three rounded but weaker secondary ribs. A few ribs arise freely on the flanks between the forked ribs. All secondary ribs trend radially on the middle of the flanks and then curve forward to form shallow chevrons on the venter.

On larger septate specimens, the ribs gradually fade out. At a diameter of $120 \mathrm{~mm}$, the primary ribs are absent and the secondary ribs are faint but form weak chevrons on the venter.

This species is characterized by having a rounded venter, a narrow umbilicus, short primary ribs, and by its adult body chamber becoming smooth.

In addition, the species may be represented by one small, laterally compressed specimen (pl. 6, figs. 5, 6), whose flanks bear ribs similar to those on the mediumsized specimens of $G$. (G.) hoveyi but whose venter bears only gently arched ribs that do not form chevrons. Those ribs, however, do become more strongly arched adorally.

$G$. (G.) hoveyi is characterized by its very narrow umbilicus, short primary ribs, and stocky lobes. Compared with $G$. (Goliathites) tumidum (Reeside), it has a smaller umbilicus, a more laterally compressed whorl section, much weaker primary ribs, fewer intercalated ribs, and its secondary ribs persist to a much larger shell diameter.

Its association with such ammonites as Pavloviceras, Pachycardioceras, Cardioceras, Scarburgiceras, Vertebriceras, and Maltoniceras shows that it is of early $\mathrm{Ox}$ fordian Age and is probably not much if at all, older than the Cardioceras cordatum zone.

Type.-Holotype, USNM 29328; paratype, USNM 32302; hypotype, USNM 303620.

Compared figured specimen.USNM 303621 (pl.6, figs. 5, 6).

Occurrences. - Redwater Shale Member of the Sundance Formation in Wyoming at USGS Mesozoic locs. $529,2386,2388,16616,19382,31382$, and 31717 ; in western South Dakota, possibly at USGS Mesozoic loc. 19570; Stump Formation in northeastern Utah at USGS Mesozoic loc. 11253 . 
Goliathiceras (Goliathites) tumidum (Reeside)

Plate 4, figures 1-14

Ammonites (Aegoceras) subtumidum Whitfield and Hovey (part), 1906, Am. Mus. Nat. History Bull., v. 22, art. 23, p. 400, pl. 60, fig. 5. Quenstedticeras? tumidum Reeside, 1919, U.S. Geol. Survey Prof. Paper 118, p. 17, pl. 4, figs. 8-10, pl. 5, figs. 3, 4.

This species is represented in the U.S. Geological Survey collections by seven specimens. It has a very stout shell, a rounded venter, whorls much wider than high, and a fairly narrow umbilicus. Its umbilical wall is vertical at its base, rounds evenly into the flanks on the inner whorls, and sharply into the flanks at a diameter of about $40 \mathrm{~mm}$.

The primary ribs on the small septate inner whorls are narrow, fairly high, and terminate in elongate swellings a little below the middle of the flanks. From the swellings pass two or three slightly weaker, radially trending secondary ribs. Some short secondary ribs are intercalated between the pairs of forked ribs. All secondary ribs bend forward slightly along the midline of the venter. A few secondary ribs fork again high on the flanks. Fairly strong ribbing persists to a diameter of $55 \mathrm{~mm}$.

The separate holotype (Reeside, 1919, p. 17, pl. 4, figs. $8-10$, pl. 5 , figs. 3,4 ) develops a smooth outer whorl at a diameter of $100 \mathrm{~mm}$. A similar smooth whorl is present on a septate specimen shown herein (pl. 4, figs. 11-13).

The species is characterized by its broad whorls, by fine ribbing; by swellings or elongate tubercles on its primary ribs, and apparently by a marked reduction in strength of ribbing on its larger septate whorls.

It resembles $G$. (Goliathites) cyclops Arkell (1947, p. 346, pl. 74, figs. 3-5) in coarseness of ribbing and in its depressed rounded whorl shape, but differs by losing its ribbing at a smaller size. Its association with such ammonites as Pavloviceras, Pachycardioceras, Cardioceras, Scarburgiceras, Vertebriceras, and Maltoniceras shows that it is of early Oxfordian age and probably not older than the Cardioceras cordatum zone.

Types.-Holotype, Am. Mus. Nat. History collection; hypotypes, USNM 303622-303627.

Occurrences.-Redwater Shale Member of the Sundance Formation in Wyoming at USGS Mesozoic locs. 16616, 19382, 29540, 29532, and 30323. Swift Formation in Montana at USGS Mesozoic locs. 22461 and 29769. The holotype was collected in Red Canyon, half a mile south of Mathias Peak, probably in sec. 34, T. 7 S., R. 3 E., Fall River County, S. Dak.

Goliathiceras (Goliathites?) subtumidum (Reeside) Ammonites (Algoceras) subtumidum Whitfield and Hovey (part), 1906, Am. Mus. Nat. History Bull., v. 22, art. 23, p. 400, pl. 60, figs. 3, 4. Quenstedtoceras? subtumidum (Whitfield and Hovey). Reeside, 1919,

U.S. Geol. Survey Prof. Paper 118, p. 16, pl. 4, figs. 1-4.

This species is not represented in the collections of the U.S. Geological Survey. The holotype specimen is characterized by coarse ribbing; a stout form; a narrow umbilicus; a venter that is sharp at a diameter of $50 \mathrm{~mm}$ but gradually becomes broadly rounded at a diameter of $70 \mathrm{~mm}$; umbilical shoulders that change in shape from evenly rounded to abrupt at a diameter of about $30 \mathrm{~mm}$; and by the presence of stocky lobes. An assignment to Goliathites is favored because the venter changes during growth from sharp to broadly rounded and because the lobes are stocky rather than elongate.

The holotype of $G$. (G. ?) subtumidum differs from $G$. (G.) tumidum Reeside by having a sharper venter. It differs from $G$. (G. ) hoveyi Reeside by having a stouter form as well as a sharper venter.

Occurrence.-Redwater Shale Member of the Sundance Formation in Wyoming near the Belle Fourche River, opposite the mouth of Inyan Kara Creek (Whitfield and Hovey, 1906, p. 401). This location is at or near USGS Mesozoic loc. 19382.

\section{Subgenus PACHYCARDIOCERAS Buckman, 1926}

Goliathiceras (Pachycardioceras) albaniense (Reeside)

Plate 10, figure 1; plate 11, figures 1, 2

Cardioceras (Pachycardioceras) albaniense Reeside, 1919, U.S. Geol. Survey Prof. Paper 118, p. 35, p. 36, pl. 23, figs. 1-3, pl. 24, figs. $1,2$.

This species is based on one specimen that shows the living chamber and part of the largest septate whorls but does not show the other septate whorls. As discussed by Reeside (1919, p. 36), the specimen resembles $G$. (P. ) incertum (Reeside) closely but "differs in its proportionately wider whorls, larger size, and more persistent and finer sculpture." It could be within the range of variation of that species, or of $G$. (P.) crassum (Reeside); it is associated with both.

Type.-Holotype, USNM 28448.

Occurrence.-Redwater Shale Member of the Sundance Formation in Wyoming at USGS Mesozoic loc. 2385.

\section{Goliathiceras (Pachycardioceras) bellefourchense (Reeside)}

Plate 6, figures 7-10

Cardioceras bellefourchense Reeside, 1919, U.S. Geol. Survey Prof. Paper 118, p. 20, pl. 11, figs. 1, 2, pl. 12, figs. 1, 2.

This species in addition to the septate holotype is now represented by a smaller specimen (pl. 6, figs. 9, 10). The general appearance of these specimens is similar to that of $G$. ( $P$. ) crassum (Reeside), but they differ by having broader inner septate whorls at diameters of about 70 to $75 \mathrm{~mm}$, an angular instead of a sharp venter, somewhat stronger ribs, and by furcation of the primary ribs a little lower on the flanks. Whether they represent a distinct species or are merely a stout, coarsely ribbed variant of $G$. $(P$. ) crassum will have to await the discovery of additional specimens.

Type.-Holotype, USNM 32321; hypotype, USNM 303628.

Occurrence.-Redwater Shale Member of the Sundance Formation in Wyoming at USGS Mesozoic locs. 2388 and 19382. Possibly represented in the Swift Formation in Montana at USGS Mesozoic loc. 29770. 
Goliathiceras (Pachycardioceras) crassum (Reeside)

Plate 8, figures 1-4; plate 9, figures 1-6

Ammonites (Amaltheus) cordiformis Meek and Hayden (part) in Whitfield and Hovey, 1906, Am. Mus. Nat. History Bull., v. 22, art. 23 , p. 401 , pls. 52,55 and 56 .

Cardioceras crassum Reeside, 1919, U.S. Geol. Survey Prof. Paper 118, p. 23, pl. 12, figs. 3, 4, pl. 13, fig. 1, pl. 14, figs. 1, 2.

This species, represented by 14 specimens, is characterized by a stout shell, a sharp unpinched venter that persists onto the largest preserved whorls, a fairly narrow umbilicus, and an umbilical wall that changes gradually during growth from gently inclined to vertical. Its body chamber is incomplete, but on the largest specimen, it occupies about one-third of a whorl.

Ribbing is coarse on the septate whorls but gradually fades on the body chamber. On the smaller septate whorls, the primary ribs are sharp, widely spaced, radially trending, and generally pass into pairs of slightly weaker secondary ribs a little below the middle of flanks. Some primary ribs remain umbranched, and some secondary ribs arise freely near the middle of the flanks. The secondary ribs curve forward high on the flanks and form chevrons on the venter.

During further growth, the ribs on septate whorls of intermediate size gradually become higher, coarser and more widely spaced. Adorally, on the largest septate whorl, the ribs become much coarser on the middle and upper parts of the flanks but gradually disappear from the lower part. Apparently, the body whorl, if preserved, must be nearly smooth.

This species differs from $Q$. $(P$.) russelli Reeside by having a sharper venter, stronger and more persistent ribbing, and stouter whorls. Its ribbing is a little coarser than that of $G$. (P. ) globosus Arkell (1947, p. 340, pl. 62, figs. 2a, b, 4a, b, 7a, b, 12a, b).

Type.-Holotype USNM 32323; hypotypes, USNM 29312, 303629-303630.

Occurrence.-Redwater Shale Member of the Sundance Formation in Wyoming at USGS Mesozoic locs. $2385,19382,29521,29535$, and 31385; in South Dakota at USGS Mesozoic loc. 10635.

Goliathiceras (Pachycardioceras) incertum (Reeside)

Plate 12, figures 1, 2; plate 13, figures 1-5; plate 14, figures 9-13 Cardioceras? incertum Reeside, 1919, U.S. Geol. Survey Prof. Paper 118 , p. 36 , pl. 20 , figs. $17-20$, pl. 21 , pl. 22, figs. $1,2$.

This species, represented by 11 specimens, has a stout shell; a narrowly rounded venter that is almost sharp on septate whorls; a fairly narrow umbilicus; a vertical umbilical wall that rounds evenly into the flanks on the smallest whorls and fairly abruptly on the outer whorls; and an adult body chamber that occupies about half a whorl and retracts slightly from the septate whorls.

The ribs on the septate whorls are low, rounded, and trend nearly radially. The primary ribs branch at onefourth to one-third the height of the flanks into two or three slightly weaker secondary ribs. Other secondary ribs arise freely on the flanks. All secondary ribs curve slightly forward on the venter and form shallow chevrons along the median line. Adorally, on the outermost septate whorl, the primary ribs gradually become faint and disappear. On the adult body whorl, the secondary ribs become very broad and faint adorally.

This species is characterized by a narrowly rounded but not sharpened venter and by low, fairly weak ribs that trend radially to near the midventral line, where they form very weak chevrons. It has much weaker ribbing than $G$. (P.) crassum (Reeside), or $G$. (P.) bellefourchense (Reeside), which are illustrated herein.

Type.-Holotype, USNM 32356; paratype, USNM 32357; hypotypes, USNM 303631-303633.

Occurrences.-Redwater Shale Member of the Sundance Formation in Wyoming at USGS Mesozoic locs. $2385,28401,30109$, and possibly at 31755 .

\section{Goliathiceras (Pachycardioceras) russelli (Reeside)}

Plate 7, figures 1-8

Cardioceras russelli Reeside, 1919, U.S. Geol. Survey Prof. Paper 118, p. 29, pl. 13, figs. 2, 3; pl. 14, figs. 3-5.

This species, represented by six specimens, has a moderately compressed discoidal shell; a sharp venter that becomes a little less sharp adorally on the largest septate whorl and on the adapical fourth of an incomplete body chamber; a fairly narrow umbilicus; a vertical to overhanging umbilical wall that passes abruptly into the flanks; a whorl section that is wider than high on the smaller septate whorls but becomes a little higher than wide on the outermost septate whorl and on the incomplete body chamber.

The primary ribs on most of the septate whorls are fairly high and trend radially on the lower third of the flanks, where they pass into two or three slightly weaker secondary ribs. A few secondary ribs arise freely near the middle of the flanks, trend nearly radially on the middle part of the flanks, and then curve gently forward to the venter, where they form chevrons. All ribs become broader and stronger during growth as far as the adapical part of the outermost septate whorl. Adorally, on that whorl, the ribs become markedly weaker but persist longest on the upper part of the flanks, where they are faintly visible on the adapical part of the body chamber.

This species is characterized by its moderately stout form, fairly strong nearly straight ribs, fairly narrow umbilicus, and the triangular cross section of its whorls.

Type.-Holotype, USNM 32324; paratype, USNM 32325; hypotypes, USNM 303634-303636.

Occurrences.-Redwater Shale Member of the Sundance Formation in Wyoming at USGS Mesozoic locs. 2388, 16616, and 20519; same member in South Dakota at USGS Mesozoic loc. 25176; in the Stump Formation in northeast Utah at USGS Mesozoic loc. 11256. Also, probably represented in Wyoming at USGS Mesozoic loc. 30306. 


\section{Genus CRRDIOCERAS Neumayr and Uhlig, 1881}

Cardioceras (Cardioceras) auroraense Reeside

Plate 16, figures 9-15

Cardioceras auroraense Reeside, 1919, U.S. Geol. Survey Prof. Paper 118 , p. 19, pl. 10, figs. 1-5.

This species is represented by 11 septate specimens that are moderate in size, discoidal, and compressed but that become stouter during growth. The best preserved specimen (pl. 16, fig. 14) at a whorl height of $29 \mathrm{~mm}$ has a whorl thickness of $21 \mathrm{~mm}$ and at a whorl height of about $47 \mathrm{~mm}$ has a whorl thickness of $45 \mathrm{~mm}$. Its umbilicus is fairly narrow and its umbilical wall is vertical and rounds abruptly into the flank. The venter bears a high compressed keel.

The septate whorls at diameters ranging from 15 to 80 $\mathrm{mm}$ bear strong moderately spaced primary ribs that curve backward on the umbilical wall and then incline slightly forward to the middle of the flanks, where they terminate in fairly strong laterally compressed tubercles. From most tubercles pass pairs of slightly weaker secondary ribs, which are separated by single secondary ribs that arise freely near or a little above the middle of the flanks. All secondary ribs curve strongly forward on the flanks and venter and form marked crenulations on the keel. The septate whorls at diameters of 80 to 100 $\mathrm{mm}$ become smooth except for faint crenulations on the keel.

The species is characterized by its coarse ribbing and fairly prominent lateral tubercles. It resembles $C$. $(C$. $)$ cordatum (J. Sowerby) as figured by Arkell (1946, p. 308, pl. 68, figs. 1-9) but differs by having more compressed whorls, a slightly narrower umbilicus, shorter primary ribs, and by lacking square shoulders and tertiary ribs.

Types.-Holotype, USNM 32319; paratype, USNM 32320; hypotypes, USNM 303637-303641.

Occurrences.-Redwater Shale Member of the Sundance Formation in Wyoming at USGS Mesozoic locs. 529, 2388, and 29529; same member in South Dakota at Mesozoic locs. 10635, 19567, and 25176.

\section{Cardioceras (Cardioceras) distans (Whitfield)}

Plate 17, figures 1-16

Ammonites cordiformis var. distans Whitfield, 1880, U.S. Geog. and Geol. Survey Rocky Mountain Region, p. 380, 381, pl. 6, fig. 25. C. distans (Whitfield). Neumayr, 1883, K. Akad. Wiss. Wien, Denkschr., Math.-Naturwiss. Klasse, v. 47, p. 302.

C. distans (Whitfield), Reeside, 1919, U.S. Geol. Survey Prof. Paper 118 , p. 24, pl. 15, figs. 18-21, pl. 16, figs. 1-6.

C. distans (Whitfield) var. depressum Reeside, 1919, U.S. Geol. Survey Prof. Paper 118, p. 24, pl. 15, figs. 22-24, pl. 16, figs. 7-11.

C. distans (Whitfield), Shimer and Shrock, 1944, Index fossils of North America, p. 585, pl. 242, figs. 3-5.

This moderate-sized species in the Western Interior region is represented by about 90 septate specimens. The whorl section is subquadrate, becomes stouter during growth, and on the largest whorls is a little wider than high. The umbilicus is moderately wide. The keel varies from sharp to pinched.
Ribs are high and sharp. Primary ribs are widely spread, incline forward slightly on the flanks, become a little stronger ventrally, and terminate near the middle of the flanks in prominent tubercles. From the tubercles arise pairs of slightly weaker secondary ribs. One or two secondary ribs arise freely between rib pairs at or a little above the middle of the flanks. All secondary ribs curve strongly forward on the venter and pass into prominent nodes on the keel. On some specimens, a few of the secondary ribs divide again at the base of the keel.

$C$. (C.) distans (Whitfield) greatly resembles the lectotype and topotype of $C$. (C. ) cordatum (J. Sowerby) figured by Arkell (1946, pl. 68, figs. 1a, b, 2a, b). It also greatly resembles other specimens that Arkell (1946, p. 308) assigned to $C$. (C.) subcordatum (Pavlow), although published under other names by Lahusen (1883, p. 50, 82, pl. 5, fig. 5a, b), Pavlow (1913, p. 48, pl. 14, fig. 5a, b), and Maire (1938, pl. 14, figs. 5a, b and 8). Apparently $C .(C$. ) distans differs by retaining fairly coarse ribbing to a diameter of at least $100 \mathrm{~mm}$, whereas $C$. $(C$.) cordatum becomes smooth at or below that diameter.

Types.-Holotype, USNM 32332; paratypes, USNM 12308, 32333-32339; hypotypes, USNM 303642-303644.

Occurrences.-Redwater Shale Member of the Sundance Formation in Wyoming at USGS Mesozoic locs. 16615, 16616, 16620, 19382, 21090, 29529, 29765, 30118, $31383,31387,31715,31716,31718,31744$, and 31752 . Same member in South Dakota at USGS Mesozoic locs. 19567, 19571 and 29538.

\section{Cardioceras (Cardioceras) hyatti Reeside}

Plate 15, figures 1-14

Cardioceras hyatti Reeside, n. sp., 1919, U.S. Geol. Survey Prof. Paper 118 , p. 26, pl. 15, figs. 1-4.

C. (Anacardioceras) hyatti Reeside. Maire, 1938, Soc. Géol. France Mém. new ser., v. 15, pts. 2 and 3, (Mém. 34), p. 75, pl. 13, fig. 2.

This species was originally described on the basis of three small septate specimens, but the collections now available include about 80 specimens, of which several show most of the adult body chamber.

The species has a compressed shell similar in shape and coiling to that of $C$. (S.) wyomingensis Reeside, as described herein. The adult body chamber occupies at least half a whorl and has a sharp compressed venter that terminates in an elevated keel.

The primary ribs are sharp and moderately spaced, start on the umbilical wall, trend radially or slightly forward on the flanks, become a little stronger ventrally, and terminate a little below the middle of the flanks. The secondary ribs arise in pairs from the primary ribs or are intercalated between the paired ribs. They are as sharp and nearly as strong as the primary ribs and maintain the same trend high on the flanks. Near the venter, the secondary ribs curve sharply forward and pass into weak crenulations on the keel. Adorally, on the body chamber, the primary ribs become weaker and more widely spaced, and the secondary ribs 
become stronger and indistinctly connected with the primary ribs.

The holotype (pl. 15, figs. 1, 2), at a maximum diameter of $45 \mathrm{~mm}$, has a whorl height of $20 \mathrm{~mm}$, a whorl thickness of $15 \mathrm{~mm}$, and an umbilical width of $12 \mathrm{~mm}$. On the largest available specimen (pl. 15, figs. 12-14) the same dimensions are $105,46,38$, and $25 \mathrm{~mm}$, respectively. Evidently the width of the umbilicus is equal to about one-fourth of the maximum diameter.

The general appearance of $C$. (C.) hyatti Reeside is similar to that of $(C).(S$. ) wyomingense Reeside. It differs by having a slightly smaller umbilicus, fairly straight ribbing on more of the flanks, stronger and sharper secondary ribs that curve forward much nearer the keel, weaker crenulations on the keel, and by lacking a faint shoulder along the margins of the venter.

Types.-Holotype, USNM 32326; hypotypes, USNM 256908, 303645-303650.

Occurrences.-Redwater Shale Member of the Sundance Formation in Wyoming at USGS Mesozoic locs. 24776, 28402, 28414, 30306, 30312, 30316, 30321, 31391, and 31715; in South Dakota at USGS Mesozoic loc. 30115. Redwater Member of the Stump Formation in northeastern Utah at USGS Mesozoic locs. 8748 and 11256. Swift Formation in Montana at USGS Mesozoic locs. 19173, 19611, and 29769.

\section{Subgenus SCARBURGICERAS Buckman, 1924 \\ Cardioceras (Scarburgiceras) americanum Reeside Plate 18, figures 9-21}

Cardioceras americanum Reeside, 1919, U.S. Geol. Survey Prof. Paper 118 , p. 18 , pl. 6 , figs. 15-20.

This species, represented in available collections by 37 specimens, of which most are very small, was illustrated originally by two fairly small septate specimens. Subsequently, a larger specimen was found that includes part of a septate whorl and about one-fourth of a larger nonseptate whorl. The septate whorl is appreciably larger than that of the holotype but bears similar ornamentation and is similar in shape and coiling.

The two specimens illustrated by Reeside (1919, pl. 16, figs. 15-19) are compressed, discoidal, and triangular in cross section. They have a sharp venter, a very narrow umbilicus, and at a diameter of about $25 \mathrm{~mm}$, their umbilical wall becomes vertical and their umbilical shoulders become sharply rounded. Ribbing is very fine but becomes stronger ventrally. The primary ribs are widely spaced, are strongest at the umbilical edge, and trend radially to the middle of the flanks, where they pass into several weaker secondary ribs. Other secondary ribs arise freely between the forked ribs. All secondary ribs curve forward high on the flanks and form crenulations on the venter.

The septate whorl that occurs with part of a nonseptate body whorl (pl. 18, figs. 16, 19-21) is triangular in cross section, a little higher than wide, and has a very narrow umbilicus. Its umbilical wall is inclined more than vertical, and its umbilical edge is abruptly rounded. Primary ribs are low and faint, trend radially or slightly forward, are strongest at the edge of the umbilicus, become weaker ventrally, and pass into pairs of secondary ribs a little below the middle of the flanks. Other secondary ribs arise freely near the middle of the flank. All ribs trend nearly straight across the flanks but at about three-fourths of the whorl height, they curve forward on the venter and form crenulations on a pinched keel.

The nonseptate body whorl of the same specimen bears very faint, broad, widely spaced primary ribs and slightly stronger more closely spaced secondary ribs that curve forward and pass into crenulations on a pinched keel. Apparently the adult whorl becomes smooth adorally except for those crenulations.

The smallest specimens (pl. 18, figs. 9-12, 17, 18), which are similar in shape and ornamentation to the larger septate specimen, differ by having a whorl section nearly as wide as high, a sharpened instead of a pinched venter, and somewhat finer ribbing.

The holotype (pl. 18, fig. 13-15) at a maximum diameter of $51 \mathrm{~mm}$, has a whorl height of $24 \mathrm{~mm}$, a whorl thickness of $16 \mathrm{~mm}$, and an umbilical width of $10 \mathrm{~mm}$. On a much larger hypotype (pl. 18, figs. 16, 19-21), the same dimensions are $106 \mathrm{~mm}, 49 \mathrm{~mm}, 39 \mathrm{~mm}$, and $24 \mathrm{~mm}$, respectively. Evidently the width of the umbilicus is equal to only one-fifth of the maximum diameter.

Types.-Holotype, USNM 32308; paratype, USNM 32309; hypotypes, USNM 303651-303653.

Occurrences.-Redwater Shale Member of the Sundance Formation in Wyoming at USGS Mesozoic locs. $16620,29523,29529$, and 29532; in Montana in the Swift Formation at USGS Mesozoic locs. 19204, 19207, 27722, 30085, 30092, 30102, and 30103.

\section{Cardioceras (Scarburgiceras) bighornense Imlay, n. sp. Plate 20, figures 1-7}

This species is represented by five specimens, of which two appear to be adults. The compressed shell has a fastigate whorl section that is a little higher than wide, a pinched venter, and an elevated keel. The body chamber, on the largest specimen, occupies nearly half a whorl.

Ribbing weakens markedly during growth. On the adapical part of the smallest specimen, the primary ribs begin on the umbilical wall, are slightly stronger near the umbilicus than they are higher on the flanks, trend radially on the flanks, and bifurcate or alternate with secondary ribs at or below the middle of the flanks. All ribs curve forward considerably high on the flanks and form marked crenulations on the sharp enter. Adorally, on the smallest specimen, all ribs become much broader, stronger, and more widely spaced; the primary ribs, in particular, become strong near the umbilicus; some primary ribs bifurcate low on the flanks; all ribs become indistinct near the venter; and the entire surface becomes marked by faint, gently flexuous striae. 
On the next larger specimen (pl. 20, figs. 4, 5) all ribs adorally gradually become lower and less conspicuous, but the primary ribs remain strongest and broadest near the umbilicus. All ribs on the venter become slightly elevated on its margins and then swing sharply forward to form weak crenulations on the keel. In addition, the entire surface bears weak striations that are particularly well shown on an external mold of a slightly larger specimen (pl. 20, fig. 2).

On the largest specimen, the ribbing gradually becomes even weaker adorally. The primary ribs become more widely spaced, the secondary ribs become faint but persist as crenulations on the keel, and faint striations are visible wherever some of the shell is preserved.

This species resembles $C$. (S.) wyomingense Reeside in shape and coiling and in the ribbing of its smallest septate whorls, but it develops entirely different ribbing on its intermediate and largest whorls.

Types.-Holotype, USNM 303654; paratypes, USNM 303655-303657.

Occurrences.-Redwater Shale Member of the Sundance Formation in Wyoming at USGS Mesozoic locs. 26717 and 29751; Swift Formation in Montana at USGS Mesozoic loc. 29770.

\section{Cardioceras (Scarburgiceras) cordiforme (Meek and Hayden)}

Plate 22, figures 22-25, 29-32)

(For references published between 1859 and 1919 see Reeside, 1919, p. 21.)

Cardioceras cordiforme (Meek and Hayden). Reeside, 1919, U.S. Geol. Survey Prof. Paper 118, p. 21, 21, pl. 7, figs. 1-6; pl. 8, figs. 1-7; pl. 9, fig. 1.

cf. Cardioceras cordiforme (Meek and Hayden). Maire, 1938, Soc. Géol. France Mém. new ser., v. 15 (Mém. 34) p. 77, pl. 8, figs. 4, 4a.

Cardioceras cordiforme (Meek and Hayden). Shimer and Shrock, 1944, Index fossils of North America, p. 585, pl. 242, figs. 1, 2.

Six specimens were assigned to this species by Reeside. Of these, the holotype as shown on his plate 7 , figures $1-4$, is a fairly large fragmentary septate specimen. A much larger septate hypotype is shown on his plate 8 , figures $1-3$ and plate 9 , figure 1 . Four very small specimens were also included in the species by Reeside (1919, pl. 7, figs. 5, 6, pl. 8, figs. 4-7).

The assignment of these four very small specimens to C. (Scarburgiceras) cordiforme (Meek and Hayden) is questioned because they are not similar morphologically to the holotype and are not associated with larger specimens that resemble the holotype. In fact, both Maire $(1938$, p. 61,62$)$ and Arkell $(1946$, p. 307$)$ consider that several other species are represented by the four small specimens. Of these, one specimen figured by Reeside $(1919$, pl. 8, figs. 4,5$)$ was assigned to $C$. (Anacardioceras) reesidei Maire $(1938$, p. 61$)$ and was considered to be the holotype by Arkell (1946, p. 307). Another specimen, figured by Reeside $(1919$, pl. 7 , fig. 5,6$)$, was apparently assigned to Cardioceras praecordatum Douvillé by Maire (1938, p. 62; Arkell, 1946, p. 307).
The writer agrees that several species are represented by the small ammonites that were assigned to $C$. (Scarburgiceras) cordiforme by Reeside. He considers 1) that one specimen figured by Reeside $(1919, \mathrm{pl} .8$, fig. 7) is an immature form of $C$. (S. ) wyomingense Reeside; 2 ) that another specimen figured by Reeside (1919, pl. 7, figs. 5, 6) is an immature form of $C$. (Cardioceras) hyatti Reeside and is very similar to a specimen of that species shown herein (pl. 15, figs. 5-7); and 3) that two specimens figured by Reeside (1919, pl. 8, figs. 4-6) belong to $C$. (Scarburgiceras) reesidei Maire.

These specific assignments are supported by the fact that the ammonite collections now available include three small- to moderate-sized specimens that are closely similar in shape and ribbing to the holotype of $C$. (S.) cordiforme (Meek and Hayden), but are quite different from the four small specimens that Reeside assigned to that species.

C. (Scarburgiceras) cordiforme (Meek and Hayden), as here redefined, has a compressed discoidal form, a triangular whorl section that is a little higher than wide in small- to medium-sized whorls but becomes a little wider than high in the larger septate specimens; a sharp venter; a fairly narrow umbilicus; a vertical umbilical wall that rounds evenly into the flanks; and nearly flat flanks that incline gently to the venter. The body chamber is unknown.

Ribbing is fairly well developed on small- and intermediate-sized specimens to a diameter of at least $85 \mathrm{~mm}$. The ribs are broad, low, fairly closely spaced, arise low on the umbilical wall, trend nearly radially on the lower two-thirds of the flanks, curve forward gently on the upper third of the flanks, and form chevrons on the venter. Some ribs bifurcate near or below the middle of the flanks, but many remain simple and are separated by short ribs that arise freely along the zone of furcation. All ribs are about equal strength at any one growth stage but become a little stronger adorally. Similar-trending but much fainter ribs occur on the adapical fourth of the largest specimen at a diameter of about $120 \mathrm{~mm}$. The rest of that septate specimen, as well as the outer whorl of the holotype, is smooth.

Types.-Holotype, USNM 203; hypotypes, USNM 32312, 303658-303660.

Occurrences.-Redwater Shale Member of the Sundance Formation in Wyoming at "southwest base of Black Hills," at USGS Mesozoic locs. 2385 and 29541; in the Redwater Member of the Stump Formation in northeast Utah at USGS Mesozoic loc. 11256.

\section{Cardioceras (Scarburgiceras) crookense Reeside}

Plate 14, figures 1-8

Cardioceras crookense Reeside. 1919, U.S. Geol. Survey Prof. Paper 118 , p. 23 , pl. 9 , figs. 2,4 .

Cardioceras crookense Reeside. Arkell, 1946, London, Paleont. Soc. A monograph on the ammonites of the English Corallian beds, pt. XI, p. 306 . 
This species, represented by 13 specimens, has a compressed shell, moderately rounded flanks, a sharp but not pinched venter, a fairly narrow umbilicus, and rounded umbilical shoulders.

Primary and secondary ribs are nearly equal in strength. The primary ribs arise on the umbilical wall, trend nearly radially or slightly forward on the lower third of the flanks, and then generally pass into pairs of secondary ribs. A few secondary ribs arise freely on the middle of the flanks. Some secondary ribs divide a little above the middle of the flanks as a trifid bundle. A few secondary ribs bifurcate near the keel. All secondary ribs on the venter curve gently forward and outnumber the primary ribs about two to one.

C. (S.) crookense differs from immature forms of $C$. (S.) cordiforme (see pl. 22, figs. 22-25) by having rounded instead of abrupt umbilical shoulders and by having ribs branching at various heights. The trifid rib bundles resemble those on $C$. (S.) martini Reeside (1919, p. 27, pl. 9, figs. 2, 5), but $C$. (S.) crookense differs by having a sharp instead of a pinched venter, by having secondary ribs that curve forward gently instead of strongly, by having ribs that are much less sharp, and by lacking prominent serrations on its venter.

Types.-Holotype, USNM 32316; hypotypes, USNM $303661,303362$.

Occurrences.-Redwater Shale Member of the Sundance Formation in Wyoming at USGS Mesozoic locs. 529,16615 , and 2382; in South Dakota at USGS Mesozoic loc. 19570 . Small pyritized specimens that probably represent $C$. (S.) crookense Reeside occur in north-central Montana at USGS Mesozoic loc. 30102.

\section{Cardioceras (Scarburgiceras?) mathiaspeakense Imlay, n. sp.}

Plate 19, figures 1-7

cf. Cardioceras (Scarburgiceras) alphacordatum Spath, in Frebold, Mountjoy, and Reed, 1959, Canada Geol. Survey Bull. 53, p. 20, pl. 1, figs. 1a, b.

cf. Cardioceras (Scarburgiceras) aff. C. (S.) alphacordatum Spath, in Frebold, 1959, Canada Geol. Survey Bull. 53, p. 21, pl. 2, fig. 1.

cf. Cardioceras (Scarburgiceras) alphacordatum Spath, in Arkell, 1946 , p. 303 , pl. 69 , figs. 8 a, b.

This species is represented by four specimens, of which one shows most of the adult body chamber. All the septate parts retain considerable shelly material. The species is moderate in size, discoidal, and compressed but becomes a little stouter on the body chamber. The umbilicus is fairly narrow on septate whorls but widens a little on the outermost whorl because of contraction of the body chamber. The umbilical wall is vertical and rounds abruptly into the flanks. The venter bears a high compressed keel. The body chamber occupies about three-fifths of a whorl and is probably nearly complete.

The small septate whorl bears robust, closely spaced primary ribs that curve backward on the umbilical wall, trend nearly radially on the lower half of the flanks, curve gently forward on the upper half, and then curve strongly forward on the venter. Most of the primary ribs remain unbranched, but a few primary ribs bifurcate near the middle of the flanks. A few secondary ribs arise freely between the primary ribs near the middle of the flanks. A few ribs bifurcate again near the keel. All ribs form fairly coarse denticulations on the keel.

The septate part of the large specimen bears weak nodes on the edge of the umbilical wall, weak radially trending striae on the lower two-thirds of the flanks, and very strong forwardly curved ribs on the upper third of the flanks. These ribs then recurve slightly, and most of them bifurcate at the margin of the keel. All of them pass into prominent nodes on a high pinched keel.

The body chamber, representing about half a whorl, retains very little shelly material and is nearly smooth, except for faint striations that arch forward on the upper part of the venter.

The holotype, at a maximum diameter of $115 \mathrm{~mm}$, has a whorl height of $52 \mathrm{~mm}$, a whorl thickness of $38 \mathrm{~mm}$, and a umbilical width of $25 \mathrm{~mm}$. At the adapical end of the body chamber, the same dimensions are $106,52,32$, and $16 \mathrm{~mm}$, respectively.

The suture line, incompletely exposed adjacent to the adult body chamber (pl. 19, figs. 1, 2), has a broad short lateral saddle and first lateral lobe, a small second lateral lobe, and small auxiliaries.

This species has ribbing similar to that on certain specimens from the Miette area in western Alberta (Frebold and others, 1959, p. 20, 21; pl. 1, figs. 1a, b; pl. 2, fig. 1) that show some resemblance to C. (Scarburgiceras ) alphacordatum Spath in Arkell (1946, pl. 69, figs. 8a, b). It differs from the specimens from western Alberta, however, by having a smaller umbilicus on the septate whorls and a retracted body chamber. Its septate inner whorls differ from the septate holotype of $C$. (S.) alphacordatum Spath by its primary ribs being much more closely spaced and mostly unbranched. The inner whorls likewise resemble those of Goliathiceras (Pachycardioceras) excavatiforme (V. Maire) (1938, p. 69 , pl. 9 , figs. 1a, b, 2a, b) in having many closely spaced unbranched primary ribs but differ by having a more compressed whorl section and a higher more compressed keel.

Types.-Holotype, USNM 303663, paratypes, USNM 303663-303665.

Occurrence.-Redwater Shale Member of the Sundance Formation in South Dakota at USGS Mesozoic locs. 19568, 25176, and 29533.

\section{Cardioceras (Scarburgiceras) schucherti Reeside} Plate 18, figures 1-8

Cardioceras schucherti Reeside, 1919, U.S. Geol. Survey Prof. Paper 118 , p. 30, pl. 11, figs. 3-5.

This species is represented by seven septate specimens. The shell is stout. Whorls are triangular in outline, a little wider than high, and have a pinched venter. 
The umbilicus is fairly narrow. The umbilical wall on outermost whorls is vertical and rounds abruptly into the flanks.

The septate whorls of small to intermediate sizes have high, narrow, widely spaced primary ribs that begin at the base of the umbilical wall and trend nearly radially to, or a little below the middle of the flanks where they terminate in laterally compressed tubercles. From these tubercles arise two or three much weaker secondary ribs. Other secondary ribs, arising freely between the tubercles, result in a total of three secondary ribs for each primary rib on the smaller septate whorls and as many as five on the larger septate whorls. All secondary ribs curve forward gently on the upper part of the flanks and on the venter and pass into crenulations on the keel.

The largest septate whorl (pl. 18, figs. 7, 8) has similar but stronger ribbing and tubercles on its adapical part and apparently much weaker ribbing and no tubercles on its adoral part. This weakening could in part be due to corrosion.

This species is characterized by its stout whorls, fairly narrow umbilicus, the presence of straight narrow, widely spaced primary ribs, which on some septate whorls terminate in tubercles, and the presence of about five much weaker secondary ribs for each primary rib. The small and intermediate-sized whorls show considerable resemblance to those on the type specimen of Cardioceras lillooetense Reeside (1919, p. 27, pl. 17, figs. 20-23), but the primary ribs and tubercles are straight, the secondary ribs are more numerous, and the species does not have weak ventral shoulders. The species also greatly resembles $C$. (S.) mirabile Arkell (1946, p. 303, text-fig. 107 on p. 304), but has much stronger primary ribs and more prominent lateral tubercles. Such coarse ribbing on its smaller septate whorls is very different from that on the holotype of $C$. (S.) alphacordatum Spath (Arkell, 1946, p. 303, pl. 69, figs. 8a, b) at a comparable size.

Types.-Holotype, USNM 32322; hypotypes, USNM 303666-303669.

Occurrerces.-Redwater Shale Member of the Sundance Formation in Wyoming at USGS Mesozoic locs. 2385, 24735, 30318, and 31382. Stump Formation in northwest Colorado at USGS Mesozoic loc. 30911. Swift Formation in Montana at USGS Mesozoic locs. 19205 and 30103.

\section{Cardioceras (Scarburgiceras) wyomingense Reeside}

Plate 21, figures 1-15

Cardioceras wyomingense Reeside, 1919, U.S. Geol. Survey Prof. Paper 118 , p. 34, pl. 15, figs. 12-17.

The original description of this species was based on parts of three septate whorls. The collections now in hand include about 120 specimens, of which several show the body chamber.
The species has a compressed shell, a fastigate whorl section that is a little higher than wide, a pinched venter, and an elevated keel. The flanks are flattened on the septate whorls but become gently rounded on the body chamber. The umbilicus is fairly narrow, and the umbilical wall is vertical and rounds abruptly into the flanks. The body chamber occupies about one-half of a whorl and is possibly complete.

The primary ribs are sharp, moderately spaced, start on the umbilical wall, trend slightly forward on the flanks, bifurcate a little below the middle of the flanks, and become distinctly stronger than the secondary ribs on the outermost septate whorl and on the body chamber. Some of the secondary ribs arise freely near the middle of the flanks. The secondary ribs are broader and a little weaker than the primary ribs but continue the same gentle forward trend to near a faint shoulder on the margin of the venter, where they curve forward more strongly and pass into fairly strong crenulations on the venter. Both primary and secondary ribs persist to the adoral end of the adult body chamber. Adorally, on the outer septate whorl and on the body chamber, all ribs become stronger and more widely spaced.

One large hypotype (pl. 21, figs. 9, 10) that includes nearly half a whorl of body chamber has a maximum diameter of $95 \mathrm{~mm}$, a whorl height of $41 \mathrm{~mm}$, a whorl thickness of $38 \mathrm{~mm}$, and an umbilical width of $26 \mathrm{~mm}$. On a medium-sized hypotype (pl. 21 , figs. 11, 13), the same dimensions are $77,31,28$, and $25 \mathrm{~mm}$, respectively. On a small hypotype (pl. 21, figs. 6,7 ), the same dimensions are $39,16,14$, and $13 \mathrm{~mm}$, respectively.

This species bears some resemblance to $C$. (Cardioceras) hyatti Reeside (1919, p. 26 , pl. 15, figs. 1-4) but differs by having a slightly wider umbilicus, secondary ribs that arch forward at a higher position on the flanks, primary ribs that are stronger relative to its secondary ribs, and a faint shoulder at the margin of the venter.

Types.-Holotype, USNM 32329; paratypes, USNM 32330 and 32331; hypotypes, USNM 303670-303676.

Occurrences.-Redwater shale Member of the Sundance Formation in Wyoming at USGS Mesozoic locs. $529,2385,11177,11179,16620,19382,24734,24736,25384$, $28815,29523,29528,29534,29541,29751,29752,29753$, $30108,30109,30114,30306,30312,30315,30322,30324-$ $30329,31390,31715,31719$ and 31750; Redwater Shale Member of the Sundance Formation in South Dakota at USGS Mesozoic locs. 19570, 27640, and 30115; Swift Formation in Montana at USGS Mesozoic locs. 19171 and 19179; Stump Formation in Utah at USGS Mesozoic loc. 11256 and in western Wyoming at USGS Mesozoic loc. 31349. Specimens compared with $C$. (S.) wyomingense occur in the Redwater Shale Member of the Sundance Formation in Wyoming at USGS Mesozoic locs. 20520, 20529, 20501, 28814, 29532, 29759, 29766, 30107, 31381, and 31390; in same member in South Dakota at USGS Mesozoic loc. 29524; in the Swift Formation in 
Montana at USGS Mesozoic locs. 19207, 22462, 30091, 30092 , and 30102.

\section{Cardioceras (Scarburgiceras) reesidei Maire}

Plate 16, figures 1-8

Cardioceras cordiforme Meek and Hayden. Reeside, 1919, U.S. Geol. Survey Prof. Paper 118, pl. 8, figs. 4a, 6. Not fig. 7.

Cardioceras (Scarburgiceras) reesidei Maire, 1938, Soc. Géol. France Mém., new ser., v. 15 (Mém. 34), p. 61, 62, pl. 7, fig. 6. Not fig. 5. Cardioceras (Scarburgiceras) cf. C. (S.) reesidei Maire. Arkell, 1946, A monograph on the ammonites of the English Corallian beds, pt. XI, p. 305-307, text-fig. 108, nos. 3-5.

This species is represented by 30 small immature ammonites that show some variation in coarseness of ribbing. One of the more finely ribbed specimens figured by Reeside (1919, pl. 8, fig. 6) was assigned by Maire (1938, p. 61) to Cardioceras precordatum Douville. One of the more coarsely ribbed specimens figured by Reeside (1919, pl. 8, figs. 4, 5) was assigned by Maire (1938, p. 61) to a new species, which Maire named Cardioceras (Anacardioceras) reesidei. Such variation in ribbing is similar to that shown in small specimens of $C$. (Scarburgiceras ) martini Reeside $(1919$, p. 27, 28, pl. 9, figs. 5-8). That species, however, at a comparable size has weaker and less flexuous primary ribs. Whether $C$. (S.) reeside $i$ is actually a distinct species needs confirmation based on additional collections that contain more mature specimens.

Types.-Holotype, USNM 32313; hypotypes, USNM 32314, 303677, 303723.

Occurrences.-Redwater Shale Member of the Sundance Formation in Wyoming at USGS Mesozoic locs. 16615,16616 , and 29541.

\section{Subgenus SCOTICARDIOCERAS Buckman, 1925 \\ Cardioceras (Scoticaxdioceras) stillwelli Reeside Plate 22, figures 11-13}

Cardioceras stillwelli Reeside, 1919, U.S. Geol. Survey Prof. Paper 118, p. 31 , pl. 6, figs. 11-14.

This species is definitely represented in available collections only by the septate holotype. That specimen has a compressed shell, rounded flanks, a fairly narrow umbilicus, and an umbilical wall that rounds gradually into the flanks. The outer whorl bears 10 broad, low, widely spaced primary ribs that trend nearly radially from the umbilical wall to near the middle of the flanks, are separated by faint striae, and pass ventrally into fine ribs. The entire upper half of the whorl bears many other faint ribs that outnumber the primary about 12 to 1 , become a little stronger ventrally, curve forward on the venter, and pass into very fine crenulations on the keel. Type.-Holotype, USNM 32307.

Occurrence.-Redwater Shale Member of the Sundance Formation in Wyoming at USGS Mesozoic loc. 16620.

Cardioceras (Scoticardioceras) cf. C. (S.) stillwelli Reeside Plate 22, figures 5-7

One very small pyritized ammonite closely resembles the holotype of $C$. (S. ) stillwelli Reeside in most features but differs by having 26 primary ribs that curve forward slightly on the lowest part of the flanks and then recurve slightly just below the middle of the flanks. Its ribbing is much finer than in $C$. (Scoticardioceras) whitfieldi Reeside (1919, p. 34, pl. 6, figs. 1-6).

Figured specimen.-USNM 303678.

Occurrences. - Swift Formation in Montana at USGS Mesozoic loc. 19579.

\section{Cardioceras (Scoticardioceras) whitfieldi Reeside}

Plate 22, figures 1-3, 8-10

Cardioceras whitfieldi Reeside, 1919, U.S. Geol. Survey Prof. Paper 118, p. 34, pl. 6, figs. 1-6.

This species is represented in available collections only by the two septate type specimens described by Reeside. It was assigned to the subgenus Scoticardioceras by Imlay (1947, p. 264) and Arkell (1956, p. 548).

The species has a compressed shell that is much higher than wide, a sharp keel, flattened flanks, a narrow umbilicus, and a vertical umbilical wall that rounds abruptly into the flanks at a diameter greater than $30 \mathrm{~mm}$.

Ribs are very fine, become finer during growth, and on the adoral third of the outer whorl of the holotype, they change to striae. Before the striae appear, the ribs bifurcate or trifurcate a little below the middle of the flanks, and some ribs arise singly between the forked ribs. All ribs incline radially or gently forward on the lower two-thirds of the flanks, bend forward more strongly to the keel on the upper third of the flanks, and then pass into very fine tertiary ribs that form crenulations on the venter.

Types.-Holotype, USNM 12294; paratype, USNM 12345.

Occurrence.-Redwater Shale Member of the Sundance Formation in Wyoming at USGS Mesozoic loc. 31716.

Cardioceras (Scoticardioceras)? cf. C. (S.) whitfieldi Reeside Plate 22, figure 4

One specimen, represented by an external mold, closely resembles the paratype of $C$. (Scoticardioceras) whitfieldi Reeside (1919, pl. 6, fig. 5) in its compressed shape and its fine ribbing but differs by having a slightly wider umbilicus and a more abruptly rounded umbilical shoulder.

Its primary ribs on the lower part of the flanks give rise to two or three secondary ribs that trend nearly radially on the flanks and then bend forward on the ventral margin. Some of the secondary ribs bifurcate again on the ventral margin. The keel is not preserved; consequently, the generic and subgeneric status of the species is uncertain.

The specimen is in a slab that contains an abundance of Buchia concentrica (Sowerby). A similar association of Buchia concentrica with Cardioceras is found in the Smithers-Hazelton area of British Columbia (Frebold and Tipper, 1975, p. 157, pl. 2, figs. 2, 4). 
Figured specimen.USNM 303679.

Occurrence.-Swift Formation in Montana near top of lower siltstone member at USGS Mesozoic loc. 27641.

\section{Subgenus CAWTONICERFS Buckman, 1923 \\ Cardioceras (Cawtoniceras) whiteavesi Reeside}

Plate 22, figures 14-21, 26-28

Cardioceras whiteavesi Reeside, 1919, U.S. Geol. Survey Prof. Paper 118 , p. 33 , pl. 17 , figs. $1-4$.

This species is represented in the U.S. Geological Survey collections by 40 specimens, of which three are as large as the holotype and one is a little larger.

The species is fairly small. In cross section, it ranges from compressed and subovate in the smallest specimens to stout and hexagonal in the larger specimens. The umbilicus is wide and has gently rounded shoulders. The keel bears fine, closely spaced tubercles which are evident at diameters of $8 \mathrm{~mm}$, or a little less. Ribs and tubercles develop on the flanks at about the same diameter and become fairly prominent within a few millimeters.

On the inner septate whorls, the primary ribs begin weakly near the umbilicus, trend nearly radially on the umbilical wall, curve slightly forward on the flanks, and terminate in sharp laterally compressed nodes near the middle of the flanks. The secondary ribs when they first appear, arise abruptly at the top of the flanks, are much weaker than the primary ribs with which they are faintly connected, outnumber the primary ribs about two to one, curve forward on the margin of the venter, and then pass faintly into nodes on the venter.

On the larger septate and nonseptate whorls, all ribs are much stronger and sharper. The secondary ribs arise mostly in pairs from the lateral tubercles, are fairly weak just above the tubercles, and pass into high, laterally compressed nodes on the margin of the venter. From these nodes pass one or two very weak tertiary ribs that incline strongly forward and pass into nodes on the venter. Some intercalated secondary ribs likewise pass into high nodes on the venter.

The ventral nodes on the small septate specimen outnumber the secondary ribs about $2 \frac{1}{2}$ to 1 and the primary ribs about 5 to 1 . On the holotype, which is less than half septate, the ventral nodes outnumber the secondary ribs about $1 \frac{1 / 2}{2}$ to 1 and the primary ribs about $3 \frac{1}{1} 2$ to 1 .

This species is characterized by its coarse sculpture, two sets of strong ribs, one set of weak tertiary ribs, sharp tubercles on the middle and upper parts of its flanks, and fairly sharp ventral tubercles that outnumber the secondary ribs.

It is distinguished from $C$. (Subvertebriceras) canadense Whiteaves by its much weaker tertiary ribs, more coarsely crenulated keel at a comparable size, and somewhat higher: and sharper secondary ribs at a comparable size.
Types.-Holotype, USNM 32340; hypotypes, USNM 303683-303686.

Occurrences.-Sundance Formation, Redwater Shale Member, in Wyoming at USGS Mesozoic locs. 16620, 28400, and 29754; Swift Formation in Montana at USGS Mesozoic locs. 19205, 19197, 30102, and 31720; also recorded from the Lillooet area, British Columbia (Reeside, 1919, p. 33).

\section{Subgenus MALTONICERAS Arkell, 1941 \\ Cardioceras (Maltoniceras) plattense Reeside}

Plate 24, figures 19, 20

Cardioceras plattense Reeside, 1919, U.S. Geol. Survey Prof. Paper 118, p. 28, pl. 9, figs. 9-12.

cf. C. (Maltoniceras) prionodes Crickmay, 1936, Geol. Soc. America Bull., v. 47, p. 559, pl. 1, fig. 3; pl. 2, fig. 13; pl. 3, fig. 3 .

This species is represented only by the holotype. It has a stout form; a triangular-shaped outer whorl that is nearly as wide as high and is weakly shouldered; a sharp keel that is not pinched; and a fairly narrow umbilicus.

The inner septate whorl, as exposed in the umbilicus of the holotype, has low, rounded, fairly widely spaced, radially trending primary ribs, more numerous but very faint secondary ribs, and distinct nodes on a crenulated keel.

The adoral fourth of the septate whorl and the adapical end of the body chamber bear similar low, widely spaced primary ribs that extend radially from the upper part of the umbilical wall across the lower fourth of the flanks. Somewhat sharper secondary ribs arise from the primary ribs in two's, or rarely three's, and some arise freely between the forked ribs. All secondary ribs trend nearly radially to a weak shoulder at about threefourths the height of the flanks, and terminate in weak elongated nodes. From these nodes pass pairs of tertiary ribs that curve slightly forward and form crenulations on the venter. These tertiary ribs outnumber the primary ribs about four to one.

Adorally, on the body chamber, the primary ribs become much weaker and more widely spaced.

The holotype of this species could represent an immature form of $C$. (M.) prionodes Crickmay (1936, p. 559, pl. 1, fig. 3), as suggested by the presence of fairly strong secondary ribs and a sharp keel.

Type.-Holotype, USNM 32318.

Occurrence.-Redwater Shale Member of the Sundance Formation in Wyoming at USGS Mesozoic loc. 2386.

\section{Cardioceras (Maltoniceras) sundancense Reeside}

Plate 23, figures 1-16

Cardioceras sundancense Reeside, 1919, U.S. Geol. Survey Prof. Paper 118 , p. 32,33 , pl. 17 , figs. $12-19$, pl. 18, figs. $1-3$.

This species, in addition to the four type specimens from the Black Hills in Wyoming, is represented by four specimens from Montana. 
The species is fairly large, discoidal, and compressed. It has a fairly narrow umbilicus. Its venter bears a keel that is pinched on its innermost whorls, is merely sharp on most whorls, and is minutely serrated. Its body chamber is incomplete but is represented by about onethird of a whorl that retracts from the inner whorls during growth. Its umbilical wall is nearly vertical basally but rounds evenly into the flanks.

Primary ribs on the inner septate whorls (pl. 23, figs. 13,14$)$ are low but distinct, bend slightly backward on the umbilical wall, incline radially, or slightly forward on the flanks, become a little stronger ventrally, bifurcate near the middle of the flanks, and are swollen or weakly tuberculate at the furcation point. Some secondary ribs arise freely between these points.

Secondary ribs on the inner septate whorls are fairly weak near the furcation points, become a little stronger ventrally, trend nearly radially on the upper parts of the flanks, curve forward, and form blunt nodes on the margins of the venter. From these nodes pass two or three very weak closely spaced tertiary ribs that curve slightly forward and pass into nodes or crenulations on the keel. Such crenulations exceed the number of primary ribs about five to one.

Adorally, on the outermost septate whorls (pl. 23, fig. 9), the tubercles on the flanks weaken and disappear. Similarly, the ribs gradually fade out adorally and ventrally except near the venter. The crenulations on the sharp venter persist, however, on the incomplete body chamber.

This species is characterized by its inner whorls bearing regular but weak ribbing; by having three sets of ribs; by the tertiary ribs being very fine; by its outer whorls becoming smooth except for a sharp serrated keel, and by its body chamber retracting from the septate whorls.

The species differs from $C$. (Maltoniceras) prionodes Crickmay (1936, p. 559, pl. 1, fig. 3, pl. 2, fig. 13, pl. 3, fig. 3) by its secondary ribs fading out at a much smaller diameter.

Types.-Holotype, USNM 29314; paratypes, USNM 32344-32346; hypotypes, USNM 303687-303691.

Occurrences.-Sundance Formation in Wyoming at USGS Mesozoic locs. 16620 and 31754; Swift Formation in Montana at Mesozoic locs. 19577, 19612, 29278, and 30087.

Cardioceras (Maltoniceras?) reddomense Imlay, n. sp. Plate 24, figures 1-18, 21

This species is represented by at least 13 specimens and perhaps also by 15 very small septate specimens.

The species is moderate in size and moderately compressed. The venter bears a keel that is narrowly rounded on small septate whorls but that becomes broadly rounded during growth and is not present on the largest preserved nonseptate whorl. The umbilical wall on the septate whorls is vertical at its base but rounds evenly into the flanks. On the adult body chamber, the umbilical wall is vertical, fairly high, and rounds rather abruptly into the flanks. The septate whorls are fastigate and as wide as high. The outermost nonseptate whorl is a little wider than high.

Primary ribs on the inner septate whorls are low, widely spaced, trend radially or slightly forward to near the middle of the flanks, where they recurve slightly and terminate in weak radially elongate tubercles, from which arise one or two secondary ribs.

Secondary ribs on the outer septate whorls are much weaker than the primary ribs, are much more numerous, arise on the flanks at, or a little above the lateral tubercles, become a little stronger ventrally, and on most specimens bear very weak elongate tubercles on the ventral margins. From these tubercles, or from the margins of the venter, arise extremely fine, closely spaced tertiary ribs that arch forward on the venter and form weak crenulations on the keel. These tertiary ribs outnumber the secondary ribs about 4 to 1 and the primary ribs about 12 to 1 .

On the nonseptate whorls, the primary ribs are broad, very low, widely spaced, incline gently forward, are strongest near the umbilicus, and become faint ventrally on the middle of the flanks. Secondary ribs are widely spaced, are fairly sharp on the upper parts of the flanks, and become stronger ventrally. All ribs fade adorally.

This species differs from $C$. (Maltoniceras) sundancense Reeside (1919, pl. 17, figs. 2-19; pl. 18, figs. 1-3) by having a stouter whorl section, a lower less serrated keel that disappears adorally on the adult whorl, weaker secondary ribs, and more numerous tertiary ribs.

Types.-Holotype, USNM 303692; paratypes, USNM 303693-303701.

Occurrences.-Swift Formation in the Pryor Mountains of south-central Montana at USGS Mesozoic locs. 19612, 29769, and 30087.

\section{Subgenus SUBVERTEBRICERAS Axkell, 1941}

\section{Cardioceras (Subvertebriceras) canadense Whiteaves}

Plate 26, figures 14-33

Cardioceras canadense Whiteaves, 1903, Ottawa Naturalist, v. 17, p. 65-67, figs. 1, 1a.

Cardioceras canadense Whiteaves. Reeside, 1919, U.S. Geol. Survey Prof. Paper 118, p. 20, pl. 17, figs. 5-11.

Cardioceras canadense Whiteaves. Frebold, 1964, Canada Geol. Survey Paper 63-4, pl. 47, figs. 3a, b.

This species is represented by about 110 specimens in available collections. These include several specimens that represent part or most of the adult body chamber, whereas the specimens described previously represent much smaller septate whorls.

The whorl section is hexagonal and a little wider than high. The umbilicus is wide and has gently rounded shoulders. The inner whorls are smooth to a diameter of about $8 \mathrm{~mm}$ and fairly smooth to a diameter of about $15 \mathrm{~mm}$. The keel is low but fairly sharp. 
On the septate whorls, the primary ribs are high, sharp and become stronger ventrally. The ribs curve backward on the umbilical wall, curve slightly forward on the flanks, and terminate at or a little above the middle of the flank in high laterally compressed tubercles. From these tubercles pass pairs of much weaker secondary ribs that become stronger ventrally, and on the ventral margin pass into sharp laterally compressed secondary tuloercles that curve forward.

From the secondary tubercles pass 2 or 3 very fine to fine tertiary ribs that swing sharply forward on the venter and pass on the keel into distinct, close, or fairly closely spaced nodes that become more widely spaced adorally. In addition, some primary ribs do not bifurcate on the flanks but all bear lateral tubercles; some secondary ribs arise freely on the upper part of the flanks; the ribbing overall varies somewhat in strength and spacing on different specimens, but on all septate specimens, the ribbing becomes higher and stronger adorally either gradually, or abruptly.

On the body chamber, in particular, all ribs and tubercles become much higher and sparser adorally; many of the secondary ribs arise freely or are indistinctly connected with the primary ribs; the tertiary ribs outnumber the primary ribs only about three to one, whereas on the septate whorls the ratio is about six to one.

C. (Subvertebriceras) canadense Whiteaves is characterized by very coarse ribbing comparable with that on $C$. (S.) zenoidae Ilovaisky, as illustrated by Arkell (1942, pl. 52, figs. 1, 2, and text-fig. 85, nos. 4-6, on p. 242).

Types.-Holotype, Geological Survey Canada No. 7437; hypotypes, USNM 32341-32343, 303702-303709.

Occurrences. - Sundance Formation, Redwater Shale Member in Wyoming at USGS Mesozoic locs. 16620, 30104, and 31753; Swift Formation in Montana at Mesozoic locs. 19612, 27722, 29542, 29769, and 30087. Probably also occurs in Montana at USGS Mesozoic locs. 19197 and 30102. Also occurs in the Fernie Group near Lillooet, British Columbia.

\section{Subgenus VERTEBRICERAS Buckman, 1920 \\ Cardiciceras (Vertebriceras) stantoni Reeside}

Plate 25, figures 16-26

Cardioceras stantoni Reeside, 1919, U.S. Geol. Survey Prof. Paper 118, p. 31, pl. 1Ei, figs. 5-8.

C. stantoni var. obesum Reeside, n. var., 1919, U.S. Geol. Survey Prof. Paper 118, p. 31, pl. 15, figs. 9-11.

This species is represented in the collections of the U.S. Geological Survey by 120 specimens from the northern part of the western interior region. Half of these specimens are from a single concretion collected near Minnekahta, S. Dak. (USGS Mesozoic loc. 19571). The specimens from that concretion, as illustrated herein, show clearly that the species varies considerably in stoutness and that some specimens are much stouter than the specimen illustrated by Reeside as $C$. stantoni var. obesum.
C. (Vertebriceras) stantoni is fairly small. Its whorl section varies from compressed to moderately stout and on most specimens is a little higher than wide. The keel is fairly sharp. The umbilicus is wide and has gently rounded shoulders.

Primary ribs are high and sharp, bend backward on the umbilical wall, curve slightly forward to the middle of the flanks, and are strongest near the middle of the flanks, where most of them bifurcate. The ventral ends of the primary ribs are not strictly tuberculate, although some are strongly swollen and could be interpreted as incipient tubercles. On specimens that bear some shelly material, the ribs are much stouter than the ribs on internal molds.

Secondary ribs arise mostly in pairs from stronger primary ribs, recurve slightly adorally on the upper parts of the flanks, become strongest on the margins of the venter, and then bend forward sharply to form crenulations on the keel. Intercalated secondary ribs become more common adorally on the body chamber.

The species is characterized by its compressed to moderately stout form, its wide umbilicus, its rounded umbilical shoulder, its sharp ribbing that is most pronounced near the middle of the flanks, and by its largest whorl bearing pronounced chevrons and a serrated keel.

Types.-Holotype, USNM 32327; paratype, 32328; Hypotypes, USNM 303710-303713.

Occurrences.-Redwater Shale Member of the Sundance Formation in western South Dakota at USGS Mesozoic locs. 19567 and 19571; in Wyoming at USGS Mesozoic locs. 529, 11177, 20529, 20531, 24776, 29531, $29749,29753,29755,31385,31715,31751$, and 31755; Redwater Member of the Stump Formation in northeast Utah at USGS Mesozoic loc. 11256; Swift Formation in Montana at USGS Mesozoic locs. 29770 and 29278.

\section{Cardioceras (Vertebriceras) haresi Reeside}

Plate 25, figures 1-10

Cardioceras haresi Reeside, 1919, U.S. Geol. Survey Prof. Paper 118, p. 25 , pl. 19, figs. 4-12.

This species is represented by 12 specimens, of which most are small and fragmentary. The whorl section is ovate and higher than wide on its inner whorls but during growth becomes quadrate and wider than high. The umbilicus is wide and has a gently rounded shoulder.

Innermost whorls are smooth. At diameters greater than $8 \mathrm{~mm}$, the whorls bear coarse primary and secondary ribs. Primary ribs curve backward on the umbilical wall, then curve gently forward to or a little above the middle of the flanks, where on the outer whorls they terminate in fairly high elongate tubercles.

Secondary ribs on the inner whorls arise mostly in pairs from the primary ribs, but many on the outer whorls arise freely on the upper part of the flanks. The ribs curve gently forward on the margins of the venter, bend sharply forward near the midline of the venter, and then rise into weak nodes along the midline. During 
growth, the secondary ribs become relatively much weaker than the coarse primary ribs.

The species is characterized by its depressed quadrate outer whorls, by those whorls attaining their greatest width ventrally, and by their ribs forming acute chevrons on the venter.

Type.-Holotype, USNM 32349; paratype, USNM 32350; hypotypes, USNM 303714-303716.

Occurrences.-Sundance Formation, Redwater Shale Member, in Wyoming at USGS Mesozoic locs. 9357, $16615,16616,19382$, and 28816 . Possibly represented in the Swift Formation in Montana at USGS Mesozoic loc. 29769.

\section{Subgenus SAGITTICERAS Buckman, 1920}

Cardioceras (Sagitticeras) obtusum Reeside

Plate 26, figures 1-7, 11-13

Cardioceras obtusum Reeside, 1919, U.S. Geol. Survey Prof. Paper 118, p. 28, pl. 20, figs. 1-6.

This species is represented by six small septate specimens from the Black Hills of eastern Wyoming and probably by two specimens from northeastern Utah. The cross section ranges from ovate in the smaller whorls to depressed ovate in the largest known whorl. At diameters greater than about $8 \mathrm{~mm}$, the whorls bear prominent, rounded, fairly widely spaced primary ribs that curve forward in the lower parts of the flanks and then recurve to the middle of the flanks where they are strongest. The secondary ribs are low, broad, and closely spaced, arise mostly in pairs from the primary ribs; curve backward on the margins of the venter; and then curve forward rather sharply to the keel, which is low, fairly smooth, and not distinctly nodose.

This species is distinguished from $C$. (Vertebriceras) haresi Reeside, with which it is associated, by having lower and broader ventral ribs, a lower, less distinct keel, and ovate instead of a quadrate whorl section, and by lacking lateral tubercles.

Types.-Holotype, USNM 32351; paratype, USNM 32352; hypotypes, USNM 303717-303719.

Occurrences.-Sundance Formation, Redwater Shale Member, in Wyoming at USGS Mesozoic locs. 16615, 16616; in the Redwater Member of the Stump Formation in northeast Utah at USGS Mesozoic locs. 6283 and 29677.

\section{Subfamily PSEUDOPERISPHINCTINAE Schindewolf, 1925 Genus GROSSOUVRIA Siemiradzkia, 1898 Subgenus POCULISPHINCTES Buckman, 1920 \\ Grossouvria (Poculisphinctes?) cf. G. (P.) trina (Buckman)} Plate 25, figures 14,15

One fragmentary ammonite from the Black Hills has a laterally compressed whorl section and a moderately wide umbilicus. Its primary ribs are sharp, fairly prominent, moderately spaced, and incline forward from the umbilical wall to a little above the middle of the flanks, where most of them give rise to pairs of secondary ribs.
The secondary ribs are much weaker than the primary ribs, outnumber them about three to one, incline slightly backward on the upper parts of the flanks, cross the venter transversely, and some terminate or join two other ribs on the venter.

This ammonite resembles the holotype of Trinisphinctes trinus Buckman (1922, v. 4, pl. 332), which Arkell (1939, p. 159) assigned to the subgenus Poculisphinctes Buckman (1920, v. 3, pl. 185). It differs from $P$. trinus (Buckman) by having much stronger ribbing and by lacking pronounced constrictions. In coiling, shape, and ribbing, the species likewise resembles Grossouvria nurrhaensis Spath (1931, pl. 4, p. 370, pl. 80, figs. 4a, b) from India but differs by having somewhat stronger ribbing. This ammonite also shows some resemblance to Decipia decipiens (Sowerby) (Arkell and others, 1957, p. L324, fig. 415) but differs in the trend of its ribs.

Figured specimen.USNM 303720.

Occurrence.-Redwater Shale Member of the Sundance Formation, about $17 \mathrm{~m}$ above base, in South Dakota at USGS Mesozoic loc. 19570. Associated ammonites include Cardioceras (Scarburgiceras) wyomingense Reeside and $C$. (S.) crookense Reeside.

\section{Grossouvria? sp.}

Plate 25, figures 11-13

One small fragment of a perisphinctid ammonite has an ovate whorl section that is a little higher than wide and bears one pronounced constriction. The primary ribs begin near the umbilicus and incline gently forward to the middle of the flanks, where most of them pass into pairs of weaker secondary ribs. Between most rib pairs are single secondary ribs that arise freely at or a little above the middle of the flanks. All secondary ribs incline forward on the flanks and arch forward slightly on the venter.

This fragment is assigned to Grossouvria rather than to Prososphinctes because its primary ribs are stronger than its secondary ribs, although it could represent an inner whorl of the species herein described as Grossouvria (Poculisphinctes?) cf. G. (P.) trina (Buckman). The specimen was collected as float together with Quenstedtoceras (Lamberticeras) collieri Reeside, Cardioceras (Maltoniceras) sundancense Reeside, and $Q$. (Pavloviceras) cf. Q. (P.) amphaloides (Sowerby).

Figured specimen.-USNM 303721.

Occurrence.-Swift Formation, 7.6-9 m above its base, in Little Rocky Mountains, Montana, at USGS Mesozoic loc. 19577.

\section{Family PERISPRINCTIDAE Steinman, 1890 Subfamily PERISPEINCTINAE Steinmann, 1890 Genus PROSOSPHINCTES Schindewolf, 1925 Prososphinctes? sp. \\ Plate 26, figures 8-10}

One small ammonite greatly resembles Prososphinctes mazuricus (Bukowski) as figured by Arkell and others (1957, p. L320, fig. 408-5a), except for being 
much smaller. Its outermost whorl is compressed and ovate, is marked by several forwardly inclined constrictions, and bears many closely spaced ribs that are very fine on the lower parts of the flanks but become broader ventrally and are most distinct on the venter. Some ribs begin near the umbilicus, some low on the flanks, and others near the middle of the flanks.

This species was collected as float together with Quenstedtoceras (Lamberticeras) collieri Reeside, $Q$. (Pavloviceras) cf. Q. (P.) amphaloides J. Sowerby, and Cardioceras (Scoticardioceras) cf. C. (S.) stillwelli Reeside.

Figured sipecimen._USNM 303722.

Occurrence.-Swift Formation, 6-71/2 m above base, in Little Rocky Mountains, Montana, at USGS Mesozoic loc. 19579.

\section{REFERENCES CITED}

Arkell, W. J., 1935-1947, A monograph on the ammonites of the English Corallian beds: London, Palaeontographical Society, $12 \mathrm{v}$. (see especially pt. 8,1942 , p. 239-254, pls. 52-55; pt. 12, 1947, p. $333-350$, pls. $71-74$ ).

1939, The ammonite succession at the Woodham Brick Company's pit, Akeman Street Station, Buckinghamshire, and its bearing on the classification of the Oxford Clay: Geological Society of London Quarterly Journal, no. 378, v. 95, pt. 2, p. 135-222, pls. 8-11.

1946, Standard of the European Jurassic: Geological Society of America Eulletin, v. 57, no. 1, p. 1-34.

1956, Jurassic geology of the world: London, Oliver \& Boyd, 806 p., 46 pls., 102 figs.

Arkell, W. J., and others, 1957, Mesozoic Ammonoidea, in Moore, R. C., ed., Treatise on invertebrate paleontology, Part L, Mollusca 4, Cephalopoda, Ammonoidea: New York and Lawrence, Kans., Geological Society of America and University of Kansas Press, p. L80-L4\$7.

Buckman, S. S., 1909-1930, Type ammonites: London, privately published, v. 1.-7.

Callomon, J. H., 1964, Notes on the Callovian and Oxfordian stages, in Maubeuge. P. L., ed., Colloque du Jurassique à Luxembourg, 1962: [Luxembourg] Institut Grand-Ducal, Section des Sciences Naturelles, Physiques, et Mathématiques, p. 269-291.

Cobban, W. A., 1945, Marine Jurassic formations of the Sweetgrass arch, Montana: American Association of Petroleum Geologists Bulletin, v. 29, no. 9, p. 1262-1303, 6 figs.

Cuppels, N. P., 1963, Geology of the Clifton quadrangle, Wyoming and South Dakota: U.S. Geological Survey Bulletin 1063-H, p. 271-321, pls. 23,24 , figs. $57-65$.

Crickmay, C. H., 1936, Study in the Jurassic of Wyoming: Geological Society of America Bulletin, v. 47, no. 4, p. 541-564, 3 pls.

Douvillé, Henri, 1912, Étude sur les Cardioceratides de Dives, Villerssur-Mer, it quelques autres gisements: Société Géologique de France Miemoires, v. 19, p. 1-77.

Enay, Raymond, Tintant, Henri, and Cariou, Elie, 1971, Les faunes Oxfordiennes d'Europe meridionale (Essai de zonation), in Colloque du Jurassique à Luxembourg, 1967: [France] Bureau de Recherches Géologiques et Minières Mémoire 75, p. 635-664.

Frebold, Hans, 1959, Marine Jurassic rocks in Nelson and Salmo areas, southern British Columbia: Canada Geological Survey Bulletin $49,31 \mathrm{p}$.

1964, Illustrations of Canadian fossils-Jurassic of western and arctic Canada: Canada Geological Survey Paper 63-4, 107 p., $51 \mathrm{pls}$.
Frebold, Hans, Mountjoy, E. W., and Reed, R. A., 1959, The Oxfordian beds of the Jurassic Fernie group, Alberta and British Columbia: Canada Geological Survey Bulletin 53, 47 p., 12 pls., 6 figs.

Frebold, Hans, and Tipper, H. W., 1970, Status of the Jurassic in the Canadian Cordillera of British Columbia, Alberta, and southern Yukon: Canadian Journal of Earth Sciences, v. 7, no. 1, p. 1-21. 1975, Upper Callovian and lower Oxfordian ammonites from southeastern Bowser Basin, British Columbia: Canadian Journal of Earth Sciences, v. 12, no. 2, p. 145-157, 2 pls., 2 figs.

Gott, G. B., and Schnabel, R. W., 1963, Geology of the Edgemont NE quadrangle, South Dakota: U.S. Geological Survey Bulletin 1063-E, p. 127-190, pls. 12-16, figs. 22-38.

Imlay, R. W., 1947, Marine Jurassic of the Black Hills area, South Dakota and Wyoming: American Association of Petroleum Geologists Bulletin, v. 31, no. 2, p. 227-273, 3 figs.

-1948, Characteristic marine Jurassic fossils from the Western Interior of the United States: U.S. Geological Survey Professional Paper 214-B, p. 13-33, pls. 4-9.

-1954, Marine Jurassic formations in the Pryor Mountains and northern Bighorn Mountains, Montana: Billings Geological Society, Guidebook, 5th Annual Field Conference, Sept. 1954, p. 54-64, 2 figs.

-1956, Marine Jurassic exposed in Bighorn Basin, Pryor Mountains, and northern Bighorn Mountains, Wyoming and Montana: American Association of Petroleum Geologists Bulletin, v. 40, no. 4, p. 562-599, 7 figs.

-1980, Jurassic paleobiogeography of the conterminous United States in its continental setting: U.S. Geological Survey Professional Paper 1062, 134 p., 33 figs.

Imlay, R. W., Gardner, L. S., Rogers, C.P., Jr., and Hadley, H. D., 1948, Marine Jurassic formations of Montana: U.S. Geological Survey Oil and Gas Investigations Preliminary Chart 32.

Knechtel, M. M., 1944, Oil and gas possibilities of the plains adjacent to the Little Rocky Mountains, Montana: U.S. Geological Survey Oil and Gas Investigations Preliminary Map 4, scale 1:48,000.

Lahusen [Laguzen], J. I., 1883-1884, Die Fauna der jurassischen Bildungen des Rjasanschen Gouvernements: Russia, Geologicheskii Komitet Mémoires, v. 1, no. 1, 94 p., 11 pls.

Love, J. D., 1958, Stratigraphy and fossils of marine Jurassic rocks along the southern margin of the Powder River Basin, Wyoming: Wyoming Geological Association, Guidebook, 13th Annual Field Conference, Powder River Basin, 1958, p. 64-70.

Love, J. D. and others, 1945, Stratigraphic sections and thickness maps of Jurassic rocks in central Wyoming: U.S. Geological Survey Oil and Gas Investigations Preliminary Chart 14.

Maire, Victor, 1938, Contributions à la connaissance des Cardiocératides: Société Géologique de France Mémoires, new ser., v. 15, no. $2 / 3$ (Mémoire 34 ), 132 p., 22 pls.

Mapel, W. J., and Pillmore, C. L., 1963, Geology of the Newcastle area, Weston County, Wyoming: U.S. Geological Survey Bulletin 1141-N, 85 p.

Maubeuge, P. L., 1975, Catalogue des ammonites du Jurassique supérieur (Callovien à Kimmeridgien) du Musée cantonal de BâleCampagne. 4 eme partie: Naturforschende Gesellschaft Baselland Tatigkeitsberichte, v. 29 (1971-1974), 338 p.

Moritz, C. A., 1951, Triassic and Jurassic stratigraphy of southwestern Montana: American Association of Petroleum Geologists Bulletin, v. 35, no. 8, p. 1781-1814.

Mudge, M. R., 1972, Pre-Quaternary rocks in the Sun River Canyon area, northwestern Montana: U.S. Geological Survey Professional Paper 663-A, 142 p., 3 pls., 4 figs.

Pavlov, A. P., 1913, Les Céphalopodes du Jura et du Crétacé inférieur de la Sibérie septentrionale: Académie Imperiale des Sciences de St. Petersbourg Mémoire, sér. 8, v. 21, pt. 4, p. 1-68, pls. 1-18.

Peterson, J. A., 1954, Marine Upper Jurassic, eastern Wyoming: American Association of Petroleum Geologists Bulletin, v. 38, no. 4 , p. $463-507,10$ figs. 
Peterson, J. A., 1957, Marine Jurassic of northern Rocky Mountains and Williston Basin: American Association of Petroleum Geologists Bulletin, v. 41 , no. 3, p. 399-440, 18 figs.

Pipiringos, G. N., 1957, Stratigraphy of the Sundance, Nugget, and Jelm Formations in the Laramie Basin, Wyoming: Wyoming Geological Survey Bulletin 47, 63 p., 5 pls., 3 figs.

-1968, Correlation and nomenclature of some Triassic and Jurassic rocks in south-central Wyoming: U.S. Geological Survey Professional Paper 594-D, 26 p., 23 figs.

Pipiringos, G. N., and Imlay, R. W., 1979, Lithology and sudivisions of the Jurassic Stump formation in southeastern Idaho and adjoining areas: U.S. Geological Survey Professional Paper 1035-C, 25 p., 16 figs.

Pipiringos, G. N., and O'Sullivan, R. B., 1976, Stratigraphic sections of some Triassic and Jurassic rocks from Douglas, Wyoming, to Boulder, Colorado: U.S. Geological Survey Oil and Gas Investigations Chart OC-69.

1978, Prinicipal unconformities in Triassic and Jurassic rocks, Western Interior United States-A preliminary survey: U.S. Geological Survey Professional Paper 1035-A, 29 p., 1 pl.
Post, E. V., 1967, Geology of the Cascade Springs quadrangle, Fall River County, South Dakota: U.S. Geological Survey Bulletin 1063-L, p. 443-504, pls. 29-31, figs. 86-91.

Reeside, J. B., Jr., 1919, Some American Jurassic ammonites of the genera Quenstedticeras, Cardioceras, and Amoeboceras, family Cardioceratidae: U.S. Geological Survey Professional Paper 118, 64 p., 24 pls.

Spath, L. F., 1927-1933, Revision of the Jurassic cephalopod fauna of Kachh (Cutch): India Geological Survey, Palaeontologia Indica, new ser., v. 9, 6 pts., 945 p., 130 pls.

Sykes, R. M., and Callomon, J. H., 1979, The Amoeboceras zonation of the Boreal upper Oxfordian: Palaeontology, v. 22, pt. 4, p. 839-903, pls. 112-121.

Whiteaves, J. F., 1903, Description of a species of Cardioceras from the Crows Nest coal fields: Ottawa Naturalist, v. 17, p. 65-67.

Whitfield, R. P., 1880, Paleontology of the Black Hills of Dakota, in Newton, Henry, and Jenney, W. P., Report on the geology and resources of the Black Hills of Dakota: U.S. Geographical and Geological Survey Rocky Mountain Region (Powell), p. 325-468, pls. 1-16.

Whitfield, R. P., and Hovey, E. O., 1906, Remarks on and descriptions of Jurassic fossils of the Black Hills: American Museum of Natural History Bulletin 22, p. 389-402. 


\section{INDEX}

[Italic page numbers indicate major references]

Page

\section{A}

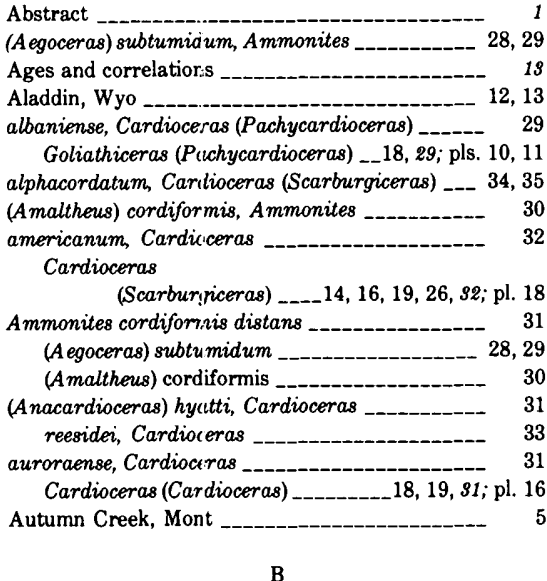

bathyomphalum, Qutnstedtoceras (Pavloviceras) -- 27 Bearpaw Mountains, Mont _-_-_-_-_-_-_-_-_-- 13, 26 Beaver Creek, S. Dak

bellefourchense, Cardioceras _-_-_-_-_-_-_--- 29 Goliathiceras

$$
\text { Bibliography }
$$
(Pachycardioceras) _-_16, 18, 19, 29, 30; pl. 6

Big Snowy Mountains, Mont

Bighorn Basin, Wyo 12,14 bighornense, Cardioceras (Scarburiniceras) _____14, 16, 17, s2; pl. 20 Scarburgiceras

Biologic analysis

Black Hills S. Dak., Wyo (Bourkelamberticerais) collieri, Quenstedtoceras _-_ 26 Bowser Basin, British̀ Columbia _-_._-_______._ 14, 15 Brush Creek, Utah Buchia concentrica ...---.---_--_-_-_.--14, 15, 36 Buffalo Gap, S. Dak

C

Camp Creek dome, M.ont _-_-_-_-_-_-_-_-_--_ 13 canadense, Cardioceras

Cardioceras (Subverte-

triceras) $13,14,16,17,19,37,38 ; \mathrm{pl} .26$

Cardioceras _-___ $4,14,15,21,28,29, s 1$ americanum -...-_- 32

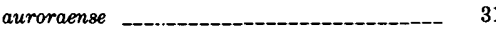

bellefourchense ...__-_____-_ $\quad 29$

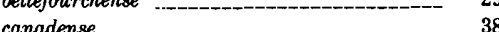

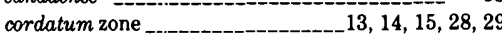

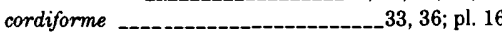
crassum _._. 30 crookense

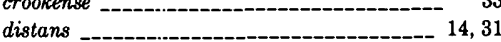

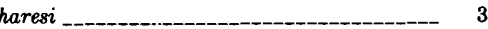

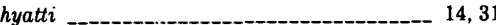
incertum - 30 latum

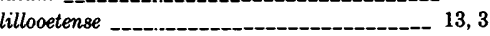

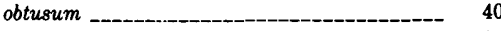
plattense 37 praecordatum ...-_-_-_-_-_-_-_-____-_ 33, 36 russelli schuchert stantoni

$$
\text { obesum }
$$

stillwelli

sundancense

whiteavesi

whitfieldi

wyomingense

(Anacardioceras) hyatti
reesidei

(Cardioceras) auroraense _ $18,19, s 1 ; \mathrm{pl} .16$

distans $17,18,19, s 1 ;$ pl. 17 cordatum

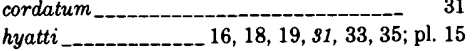
subcordatum _-_-_-_-_-_-_-_-_-_-_._- 31 $\mathrm{sp}$

(Cawtoniceras) whiteavesi _._____ 13, 14, 16, 17, 19, s7; pl. 22 (Maltoniceras)

Cardioceras (Maltoniceras) plattense18, 37 ; pl. 24 prionodes
reddomense sundancense

$41 ; \mathrm{pl} .23$

(Pachycardioceras) albaniense (Sagitticeras) obtusum _-_ 16, 19, 40; pl. 26

(Scarburgiceras) alphacordatum 34,35 americanum_-_-_-_-_14, 16, 19, 26, $s 2$; pl. 18 bighornense________ 14, 16, 17, s2; pl. 20 cordiforme ________ $16,18,19, s s, 34 ; \mathrm{pl} .22$ crookense ------_ 14, 16, 18, 19, ss, 40; pl. 14 martini__-__-_______-_-_-_ 34,36 mathiaspeakense _-__________-_-_ 19, s4; pl. 19 mirabile schucherti $13,16,18$, s4; $\mathrm{pl}, 18$ syomingense__ $14,16,17,18,19,31,32,33,35$, 40; pl. 21 sp

Scoticardioceras) stillwelli______ $16,19,26, s 6,41 ;$ pl. 22 $14,16,19,36$; pl. 22 (Subvertebriceras) canadense renaidae 39

(Vertebriceras) haresi__.___ $16,18,19,39,40 ;$ pl. 25 stantoni $13,16,17,18,19,89 ; \mathrm{pl} .25$

(Cardioceras) auroraense, Cardioceras__18, 19, s1; pl. 16 cordatum, Cardioceras____________ 31 distans, Cardioceras_-_-_17, 18, 19, s1; pl. 17 hyatti, Cardioceras____16, 18, 19, 31, 33, 35; pl. 15 subcordatum, Cardioceras sp., Cardioceras___- 16 Cardioceratidae _..._- 4, 15, 26 Cardioceratinae -_- 4,26 (Cawtoniceras), Cardioceras -13, 14, 16, 17, 19, s7; pl. 22 whiteavesi, Cardioceras _ 13, 14, 16, 17, 19, 37, pl. 22

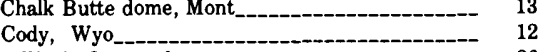
collieri, Quenstedtoceras

Quenstedtoceras (Bourkelamberticeras) 26 (Lamberticeras) $\quad 16,26,40,41 ; \mathrm{pl} .1$ concentrica, Buchia cordatum, Cardioceras (Cardioceras) cordatum, zone, Cardioceras cordatum zone, Cardioceras_____________ 13, 15, 28, 29
cordiforme, Cardioceras Cardioceras (Scarburgiceras)_-_-_16, 18, 19, ss, 34; pl. 22
Cardioceras-Continued

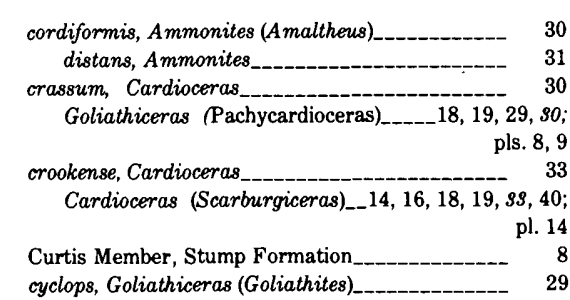

D

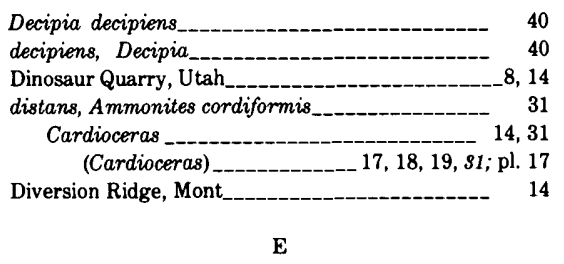

Ephraim Conglomerate European ainmonite zones. See name of particular zone. excavatiforme, Goliathiceras (Pachycardioceras) _- 34 Evanston, Wyo

Faunal setting

Flat Top anticline, Wyo

Freezeout Mountains, Wyo

G

Geographic distribution 15 Glacier National Park, Mont globosus, Goliathiceras (Pachycardioceras) _____._ 30 Goliathiceras 4,28 (Goliathiceras) suspectum _-_-_-_18, 19, 28; pls. 2, 3 $\begin{array}{ll}\text { (Goliathites) -_- } & \\ \text { cyclops } & \end{array}$ hoveyi subtumidum - 19,29 tumidum - $14,16,19,28,29 ;$ pl. 4 (Pachycardioceras) albaniense -_-_-_-_18, 29 ; pls. 10,11 bellefourchense --_-_-_16,18, 19, 29,30; pl. 6 crassum -_-_18, 19, 29, 30 ; pls. 8, 9 excavatiforme --_-_-_- 34 incertum 17, 18, 19, 29, s0; pls. 12, 13, 14 russelli $16,18,19,90 ; \mathrm{pl} .7$ (Goliathiceras) suspectum Goliathiceras

$18,19,28 ;$ pls. 2,3 (Goliathites), Goliathiceras _..._-____._14, 15, 28, 29 (Goliathites) cyclops, Goliathiceras hoveyi, Goliathiceras ___ 18, 19, 28, 29; pls. 5, 6 Quenstedtoceras _- 16, 28 subtumidum, Goliathiceras _.-_-_-_-_-_.-. 19, 29 tumidum, Goliathiceras _-_-_14, 16, 19, 28, 29; pl. 4 Great Falls, Mont Greybull, Wyo Grossouvria -

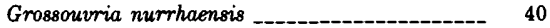

sp -_-_-_-_-_ 16, 26, 40; pl. 25 (Poculisphinctes) -
trina -190 Gryphaea 21 
39

haresi, Cardiocera

Cardioceras (Vertebriceras) _ 16, 18, 19, 39, 40; pl. 25 hoveyi, Goliathiceras (Goliathites) 18, 19, 28, 29; pls. 5, 6 Quenstedtoceras

(Goliathites) _-_-_-_..- 16,28

Hulett Sandstone Member, Sundance Formation _- 12 hyatti, Cardioceras 14,31

Cardioceras (Anacardioceras) _ (Cardioceras) ____16, 18, 19, s1, 33, 35; pl. 15

I

incertum, Cardioceras

30

Goliathiceras (Pachycardioceras) _._17, 18, 19, 29, so;

Introduction pls. $12,13,14$

\section{L}

Lak Member, Sundance Formation lamberti, Quenstedtoceras (Lamberticeras) lamberti zone, Quenstedtoceras _-_-_-_-_-_-_ 13 (Lamberticeras), Quenstedtoceras _-_______ 4, 13, 21, 26 collieri, Quenstedtoceras ________16, 26, 40, 41; pl. 1 lamberti, Quenstedtoceras Lander anticline, Wyo _._._- 14 Lander, Wyo

latum, Cardioceras (Pavloviceras) 16, 18, 19,27, 28; pl. 1 lillooetense, Cardioceras _..-_-___-_ 13, 35 Lily Park, Colo

Little Brush Creek, Utah

Little Rocky Mountains, Mont __ 13, 26

Little Sheep Mountain, Wyo _._-_-_-_-_-_-_ 14

Lovell, Wyo

M

(Maltoniceras), Cardioceras _-_-_-_-_-_4, 14, 28, 29, 37 plattense, Cardioceras _-_-_-_-_._-_ 18 ; pl. 24 prionodes, Cardioceras ___________ 37, 38 reddomense, Cardioceras 16,$38 ; \mathrm{pl} .24$ sundancense, Cardioceras _.....13, 16, 17, 19, 26, s7, $38,40,41 ;$ pl. 23

mariae zone, Quenstedtoceras _-._-_-_--_--_--- 13 martini, Cardioceras (Scarburgiceras) mathiaspeakense, Cardioceras (Scarburgiceras) _-____-_19, s4; pl. 19 mazuricus, Prososphinctes _._.__________ 40

McCoy Creek, Idaho Minnekahta, S. Dak minnekahtense, Quenstedtoceras (Pavloviceras) ___27; pl. 1 mirabile, Cardioceras (Scarburgiceras) _-_.-_ 35 Morrison Formation $4,8,12$

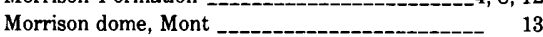

N

nurrhaensis, Grossouvria 40

\section{o}

obesum, Cardioceras stantoni obtusum, Cardioceras _-_._-_-_-_-_ 40 Cardioceras (Sagitticeras) -_-_-_-_16, 19, 40; pl. 26 omphaloides, Pavloviceras _-_-_-___-_-_-_.- 26

Quenstedtoceras (Pavloviceras) _-_-_-_16, 26, 40; pl. 2

$$
\mathbf{P}
$$

(Pachycardioceras), Goliathiceras

$4,14,28,29$ albaniense, Cardioceras

albaniense, Goliathiceras _-_-_-_-_18, 29; pls. 10, 11 bellefourchense, Goliathiceras _16, 18, 19, 29, 30; pl. 6 crassum, Goliathiceras _--_--18, 19, 29, s0; pls. 8, 9 excavatiforme, Goliathiceras --- 34

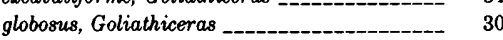
incertum, Goliathiceras -------_17, 18, 19, 29, so; pls. $12,13,14$
Paleogeography

Pavloviceras omphaloides

26

(Pavloviceras), Quenstedtoceras _-_-_-_4, 15, 26, 28, 29 bathyomphalum, Quenstedtoceras _-____-_ 27 latum, Quenstedtoceras _-_-_-_16, 18, 19, 27, 28; pl. 1 minnekahtense, Quenstedtoceras ________ 27; pl. 1 omphaloides, Quenstedtoceras _-_____-_16, 26, 40; pl. 2 schaunburgensis, Quenstedtoceras _._._._._._. 27 stibarum, Quenstedtoceras

Peoa, Utah

Perisphinctes plicatilis zone _.....___._._._. 13, 14,

Perisphinctidae _._._._._. 4, 40

Perisphinctinae -_-_-_-_-_-_-_ 4, 40

Phylloceras -

Pine Butte Member, Sundance Formation $\quad 12$

Piper, Mont _-____________________________ 14

plattense, Cardioceras

Cardioceras (Maltoniceras)

plicatilis zone, Perisphinctes _-________-_13, 14, 15

(Poculisphinctes), Grossouvria _-_____-_ 4, 15, 40

trina, Grossouvria

praecordatum, Cardioceras _-_-_-_-_-_-_-- 33, 36

prionodes, Cardioceras (Maltoniceras) 37, 38

Prososphinctes _-_._._. $4,13,15,40$ mazuricus $\mathrm{sp}$

16,$40 ;$ pl. 26

Pryor Mountains, Mont

$5,12,14$

Pseudoperisphinctinae

$-4,40$

\section{Q}

Quenstedticeras? tumidum

Quenstedtoceras

collieri

hoveyi

lamberti zone

mariae zone

subtumidum

suspectum

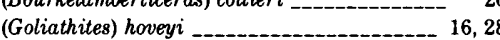

(Lamberticeras) - 13, 26

collieri _-__-___________-_16, 26, 40, 41; pl. 1 lamberti

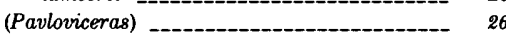
bathyomphalum latum _-_._._16, 18, 19, 27, 28; pl. 1 minnekahtense $27 ; \mathrm{pl} .1$ omphaloides _-_ schaunburgensis

stibarum 27
28

Red dome, Mont 12,14 reddomense, Cardioceras (Maltoniceras) 16, 38; pl. 24 Redwater Member, Stump Formation ___ $1,8,14$ Redwater Shale Member, Sundance Formation $1,8,12,14$ reesidei, Cardioceras (Anacardioceras) 33,36 Cardioceras (Scarburgiceras) $\quad 19,33,36 ; \mathrm{pl} .16$ Rierdon Formation

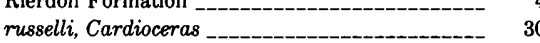

Goliathiceras (Pachycardioceras) _16, 18, 19, s0; pl. 7

(Sagitticeras), Cardioceras $-4,14,40$ obtusum, Cardioceras $16,19,40 ; \mathrm{pl} .26$ Sawtooth Range, Mont Scarburgiceras bighornense (Sarourgiceras), Cardioceras _--4, 13, 14, 15, 28, 29, 32 alphacordatum, Cardioceras _- 34, 35 americanum, Cardioceras 14, 16, 19, 26, 32; pl. 18 bighornense, Cardioceras _.___14, 16, 17, $32 ; \mathrm{pl} .20$ cordiforme, Cardioceras _--_16, 18, 19, 33, 34; pl. 22 crookense, Cardioceras _-_14, 16, 18, 19, ss, 40; pl. 14 martini, Cardioceras _34, 36 mathiaspeakense, Cardioceras _---_-_19, s4; pl. 19 mirabile, Cardioceras reesidei, Cardioceras $-19,33, s 6 ;$ pl. 16 schucherti, Cardioceras _-_-_-_13, 16, 18, s4; pl. 18
(Scarburgiceras), Cardioceras.- Continued wyomingense, Cardioceras _14, 16, 17, 18, 19, 31, 32 $33,35,40 ;$ pl. 21

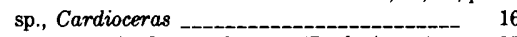
schaunburgensis, Quenstedtoceras (Pavloviceras) _- 27 schucherti, Cardioceras (Scoticardioceras), Cardioceras ___-_-___ 4, 36 stillwelli, Cardioceras _._-_-_16, 19, 26, s6, 41; pl. 22 whitfieldi, Cardioceras _-____-_14, 16, 19, 36; pl. 22 Sheridan arch

Shoshone River, Wyo

Snake River, Idaho

Spearfish, S. Dak

stantoni, Cardioceras Cardioceras (Vertebriceras) -_-_-_-_13, 16, 17, 18 19,$99 ;$ pl. 25

$\begin{array}{ll}\text { stantoni obesum, Cardioceras } & 39 \\ \text { stibarum, Quenstedtoceras (Pavloviceras) } & 28\end{array}$ stibarum, Quenstedtoceras (Pavloviceras) ------- 28 Cardioceras (Scoticardioceras)

pl. 22

Stratigraphic summary -_-_-_.-- 1,8 Stump Formation subcordatum, Cardioceras (Cardioceras) ---_----- 28, 29 Goliathiceras (Goliathites) 19,29 Goliathiceras (Goliathites) --_-_-_-19, 29
Quenstedtoceras

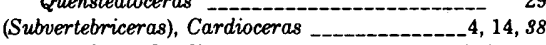
canadense, Cardioceras _-_.- 13, 14, 16, 17, 19, 37, s8; $\begin{aligned} & \text { pl. } 26\end{aligned}$ Summit, Mont Sun River Canyon, Mont

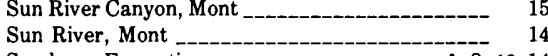
Sundance Formation sundancense, Cardioceras Cardioceras (Maltoniceras) _-_._13, 16, 17, 19, 26, s7, sespectum, Goliathiceras suspectum (Goliathiceras) _-_-_-_18, 19, 28; pls. 2, 3 $\begin{array}{rr}\text { Quenstedticeras } & 28 \\ \text { Sweetgrass arch, Mont } & 4,5\end{array}$ Sweetgrass arch, Mont Systematic descriptions $\mathrm{T}$

Tensleep, Wyo 12 trina, Grossouvria (Poculisphinctes) - 19, 40; pl. 25 Trinisphinctes trinus trinus, Trinisphinctes tumidum, Goliathiceras (Goliathites) Quenstedticeras

$\mathrm{U}$

Uinta Mountains, Utah 8 V

Vernal, Utah

(Vertebriceras), Cardioceras --------4, 14, 15, 28, 29, 89 haresi, Cardioceras _--_--_16, 18, 19, s9, 40; pl. 25 stantoni, Cardioceras _._. 13, 16, 17, 18, 19, s9; pl. 25

W

whiteavesi, Cardioceras Cardioceras (Cawtoniceras) whitfieldi, Cardioceras 37 ; pl. 22 Cardioceras (Scoticardioceras) _-_14, 16, 19, s6; pl. 22 Williston Basin Williston Basin
Wind River Basin, Wyo Windy Hill Sandstone Member, Sundance Formation ____________, 8,14 Wyoming Range, Wyo

uyomingense, Cardioceras

Cardioceras (Scarburgiceras) _-_-_14, 16, 17, 18, 19, $31,32,33,35,40 ;$ pl. 21

Z

zenaidae, Cardioceras (Subvertebriceras) 39

Zortman Butte, Mont

U.S. GOVERNMENT PRINTING OFFICE: $1982-361-614 / 14$ 


\section{PLATES $1-26$}

Contact photographs of the plates in this report are available, at cost, from U.S. Geological Survey Library, Federal Center, Denver, Colorado 80225. 


\section{PLATE 1}

[Figures natural size unless otherwise indicated]

FIgURES 1-12. Quenstedtoceras (Lamberticeras) collieri Reeside (p. 26)

1, 2. Hypotype, USNM 303605, from USGS Mesozoic loc. $19210(\times 2)$.

3. Hypotype, USNM 303724, from USGS Mesozoic loc. $19210(\times 2)$.

4. Hypotype, USNM 303604, from USGS Mesozoic loc. 19577.

5, 6. Hypotype, USNM 303606, from USGS Mesozoic loc. 19210

7. Hypotype, USNM 303603, from USGS Mesozoic loc. 19577.

8. Hypotype, USNM 303602, from USGS Mesozoic loc. 19577.

9, 10. Hypotype, USNM 303601, from USGS Mesozoic loc. 19577.

11, 12. Hypotype, USNM 104137, from USGS Mesozoic loc. 19577.

13, 15-20, 22-24, 29. Quenstedtoceras (Pavloviceras) latum (Reeside) (p. 27)

13, 29. Mostly nonseptate hypotype, USNM 303612, from USGS Mesozoic loc. 30341.

15, 16. Hypotype, USNM 303613, from USGS Mesozoic loc. 16616.

17, 18. Paratype, USNM 32353, from USGS Mesozoic loc. 16615.

19, 20. Holotype, USNM 32354, from USGS Mesozoic loc. 19382.

22-24. Hypotype, USNM 303611, from USGS Mesozoic loc. 30341

14, 21. Quenstedtoceras (Pavloviceras) cf. Q. (P.) latum (Reeside) (p. 27)

Ventral and lateral views of immature specimen ( $\times 3)$, USNM 303614, from USGS Mesozoic loc. 19204.

25-28, 30. Quenstedtoceras (Pavloviceras) minnekahtense Imlay, n. sp. (27)

25, 26. Paratype, USNM 303616, from USGS Mesozoic loc. 16616.

27, 28, 30. Holotype, USNM 303615, from USGS Mesozoic loc. 19571. 


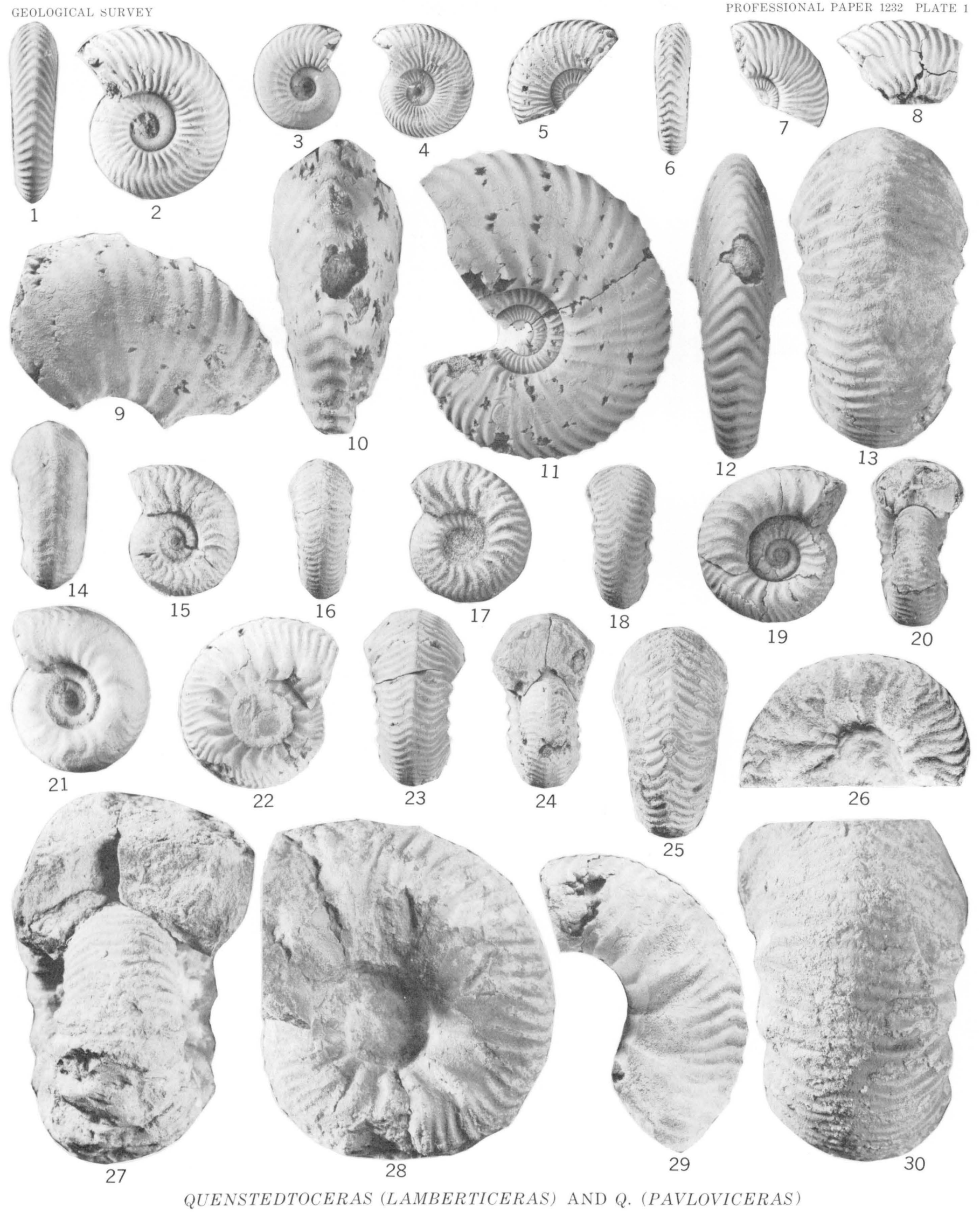


PLATE 2

[Figures natural size unless otherwise indicated]

Figures 1-8. Quenstedtoceras (Pavloviceras) cf. Q. (P.) omphaloides (J. Sowerby) (p. 26)

1, 2. Nearly smooth, immature specimen ( $\times 2$ ), USNM 303607, from USGS Mesozoic loc. 19204

3, 4. Slightly larger immature specimen ( $\times 2$ ), USNM 303610, from USGS Mesozoic loc. 19577.

5, 6. Fairly strongly ribbed specimen, USNM 303609, from USGS Mesozoic loc. 19579.

7, 8. Strongly ribbed septate specimen, USNM 303608, from USGS Mesozoic loc. 19579

9, 10. Goliathiceras (Goliathiceras) suspectum (Reeside) (p. 28)

Lateral and apertural view of a large septate specimen from which nearly half of a whorl has been removed. Hypotype, USNM 303617, from USGS Mesozoic loc. 30336. 


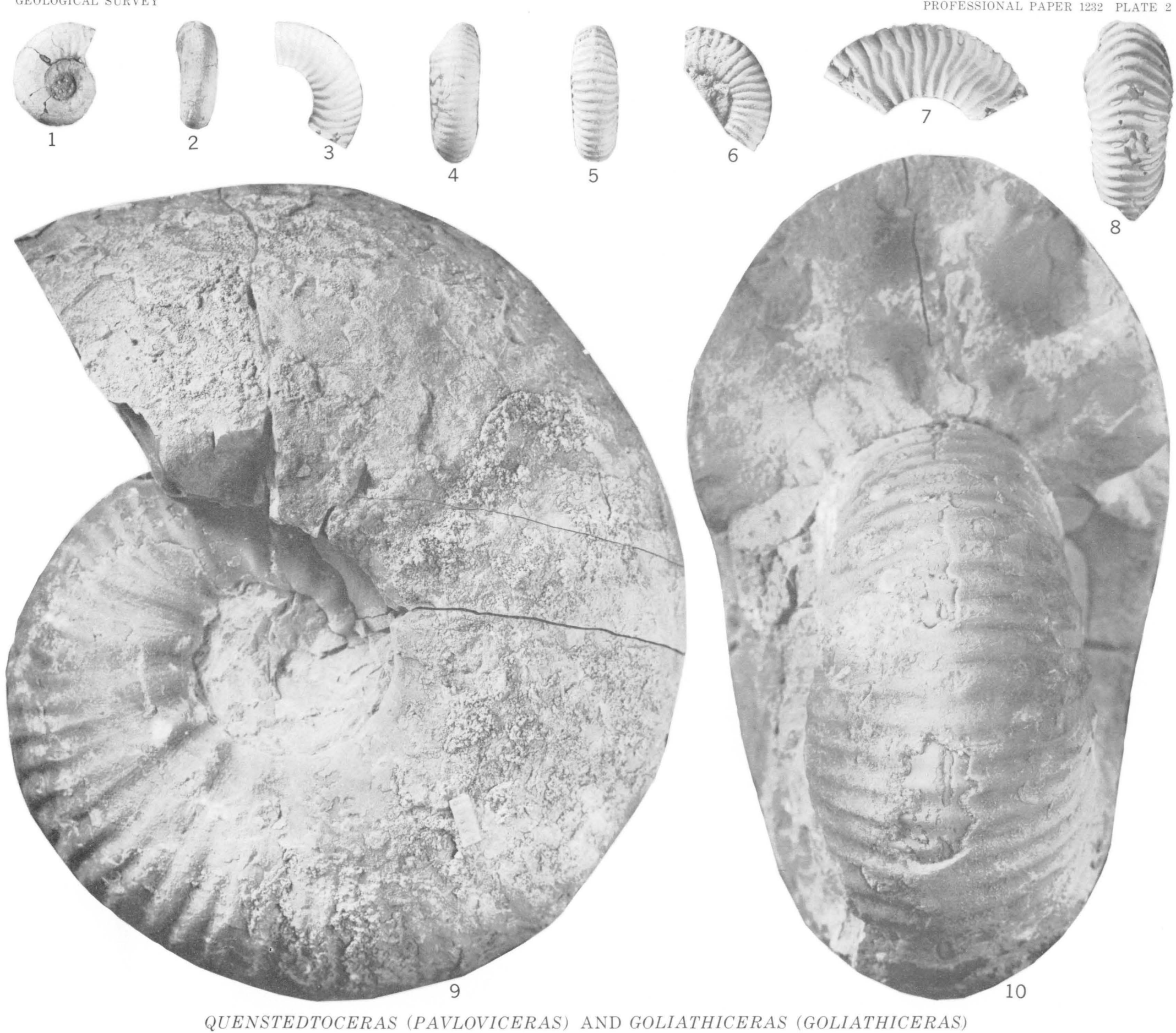


PLATE 3

[All figures are natural size]

FIGURES 1-7. Goliathiceras (Goliathiceras) suspectum (Reeside) (p. 28)

1, 2. Lateral and ventral views of inner whorls of the holotype, USNM 32304, from USGS Mesozoic loc. 529.

3, 4. Hypotype, USNM 303619, from USGS Mesozoic loc. 30110.

5-7. Hypotype, USNM 303618, from USGS Mesozoic loc. 29535. 


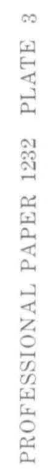
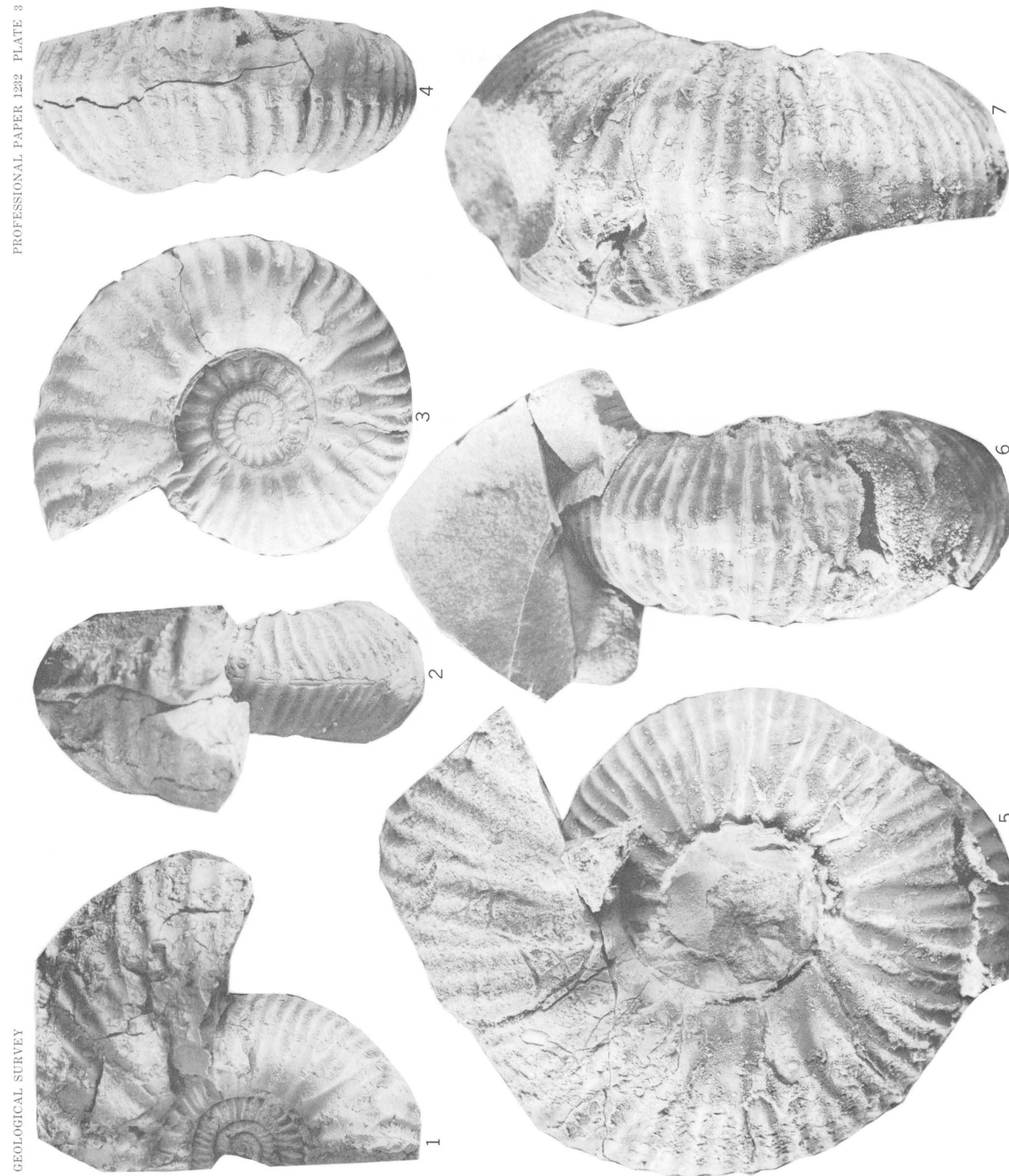

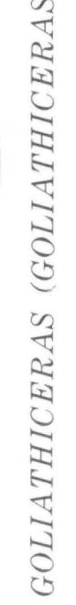




\section{PLATE 4}

[All figures are natural size]

FIGURES 1-14. Goliathiceras (Goliathites) tumidum (Reeside) (p. 29)

1-3. Hypotype, USNM 303625, from USGS Mesozoic loc. 16616.

4. Hypotype, USNM 303624, from USGS Mesozoic loc. 30323.

6. Hypotype, USNM 303626, from USGS Mesozoic loc. 30323.

7, 8. Hypotype, USNM 303623, from USGS Mesozoic loc. 22461.

9, 10, 14. Apertural, ventral and lateral views of hypotype USNM 303627, from USGS Mesozoic loc. 29769.

11-13. Lateral and ventral views of inner whorl and ventral view of outer whorl. Hypotype, USNM 303622, from USGS Mesozoic loc. 29540. 
GEOLOGICAL SURVEY
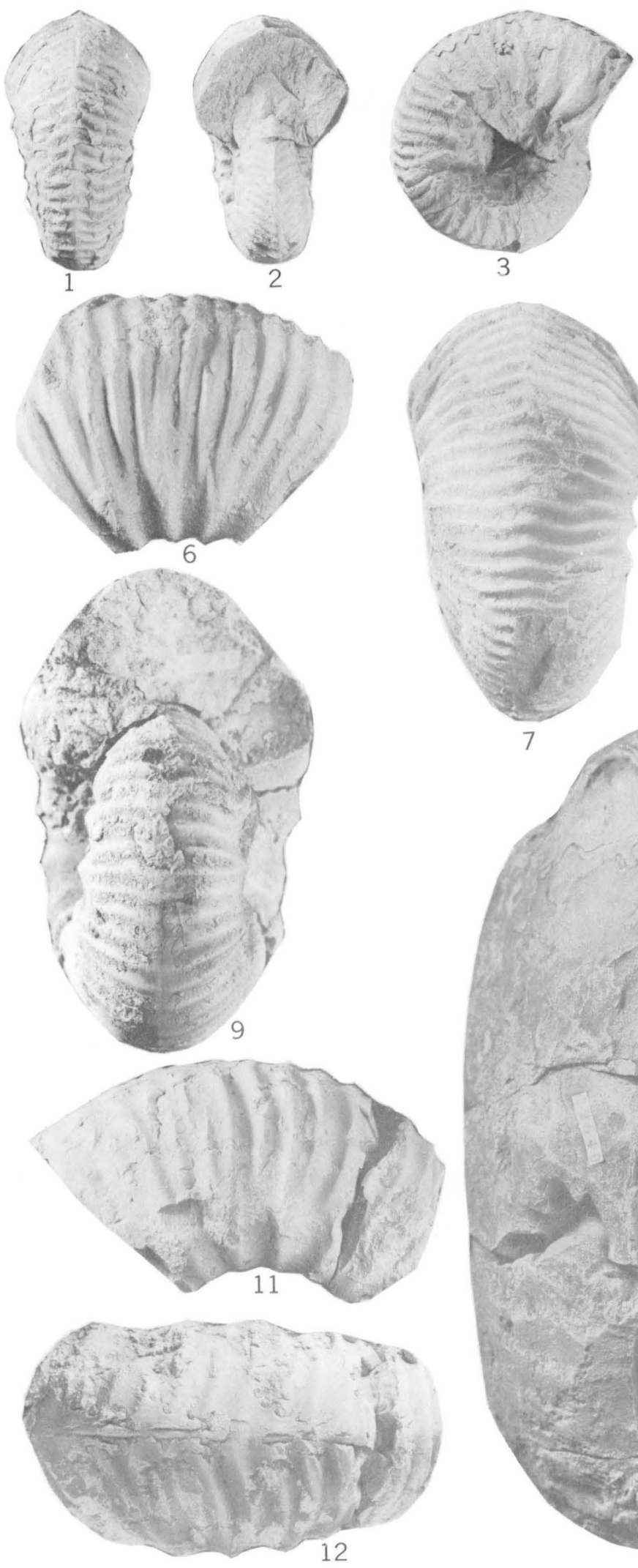

PROFESSIONAL PAPER 1232 PLATE 4
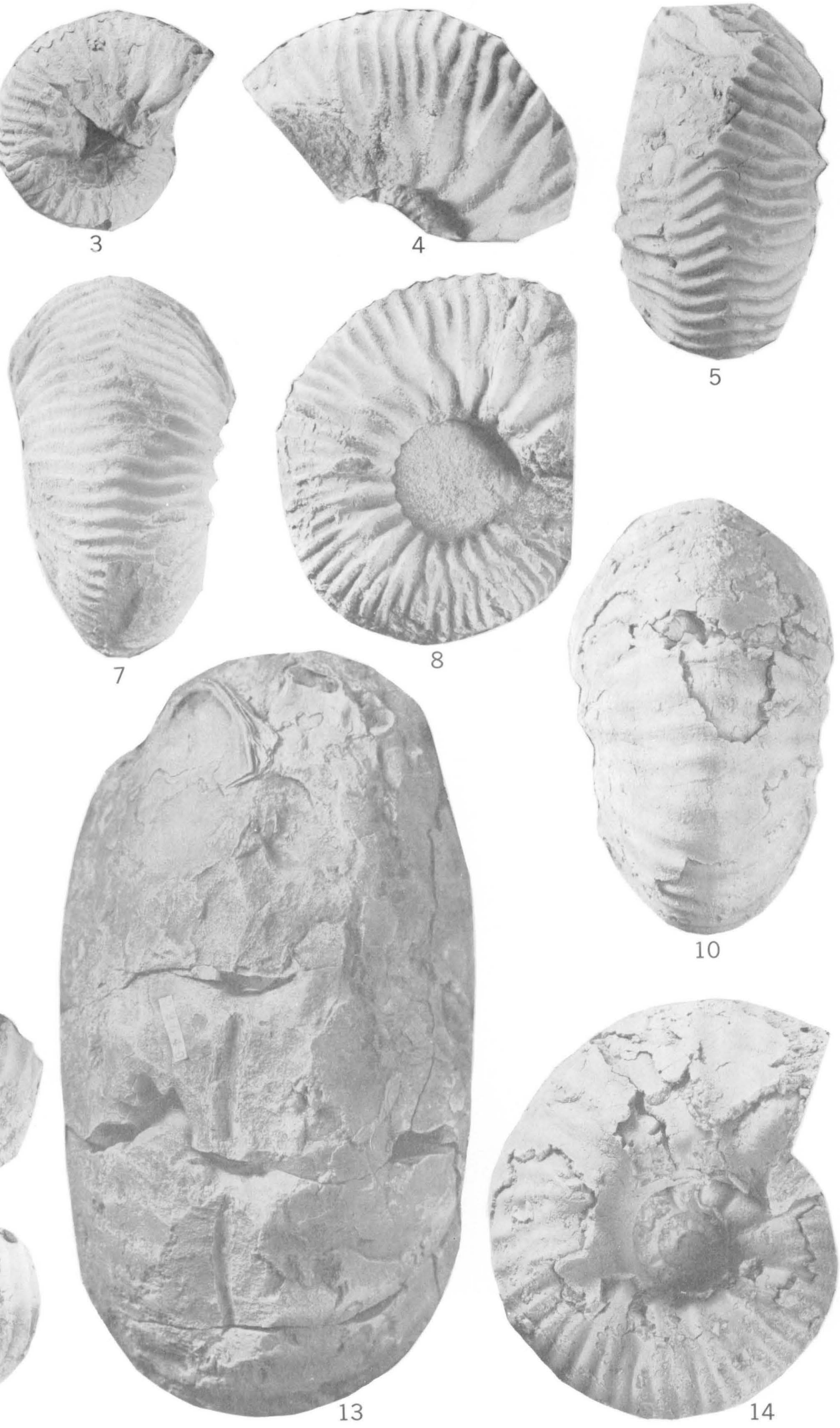

GOLIATHICERAS (GOLIATHITES) 


\section{PLATE 5}

[All figures are natural size]

FIGURES 1, 2. Goliathiceras (Goliathites) hoveyi (Reeside) (p. 28)

Lateral and apertural views of paratype, USNM 32302, from USGS Mesozoic loc. 529. 


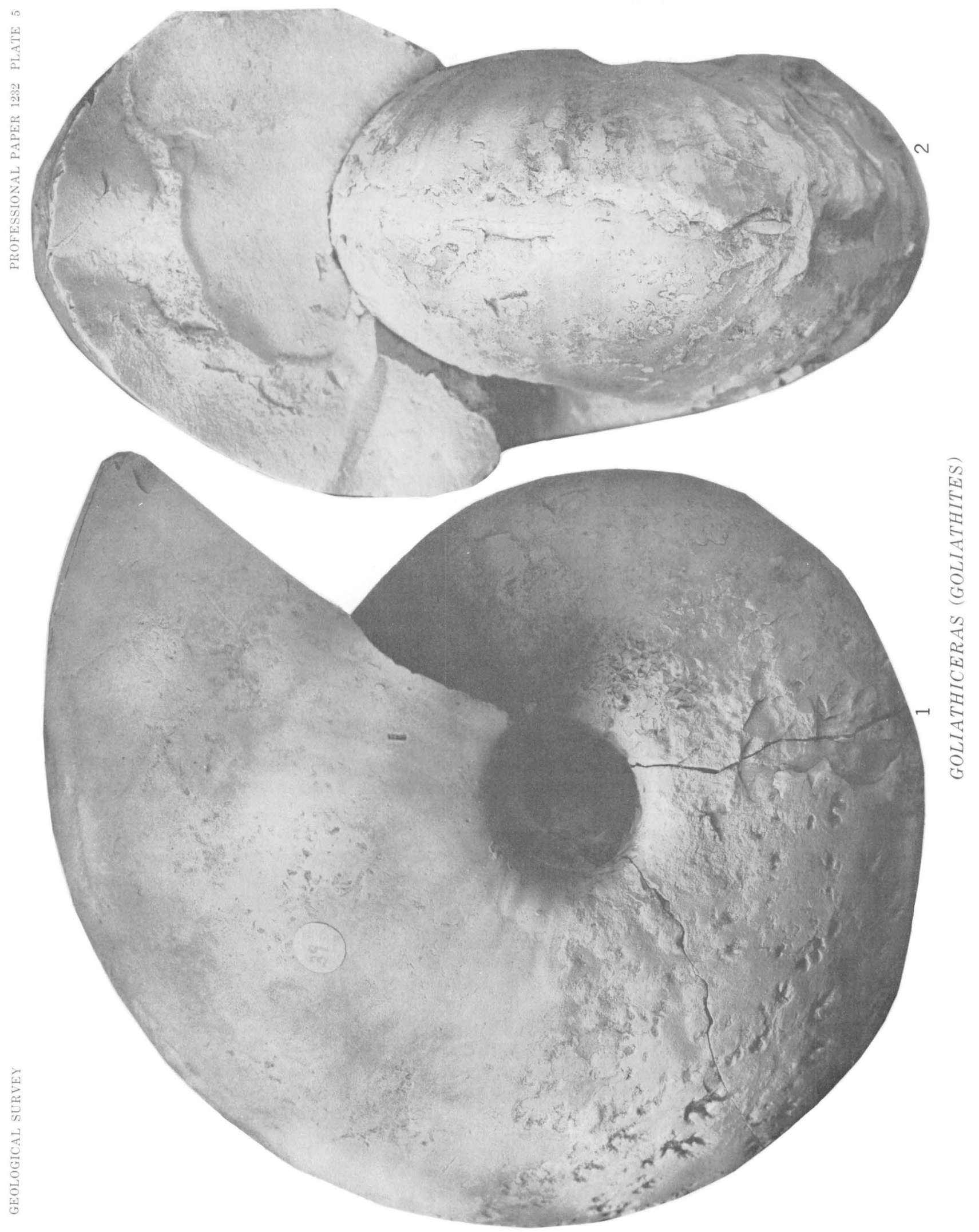




\section{PLATE 6}

(All figures are natural size]

FIGURES 1-4. Goliathiceras (Goliathites) hoveyi (Reeside) (p. 28)

1-3. Ventral, apertural and lateral views of hypotype, USNM 303620, from USGS Mesozoic loc. 31382

4. Holotype, USNM 29328, from USGS Mesozoic loc. 529

5, 6. Goliathiceras (Goliathites) cf. G. (G.) hoveyi (Reeside) (p. 28)

Views of immature specimen, USNM 303621, from USGS Mesozoic loc. 19570.

7-10. Goliathiceras (Pachycardioceras) bellefourchense (Reeside) (p. 29)

7, 8. Lateral and ventral views of holotype, USNM 32321, from USGS Mesozoic loc. 19382.

9, 10. Hypotype, USNM 303628, from USGS Mesozoic loc. 2388 


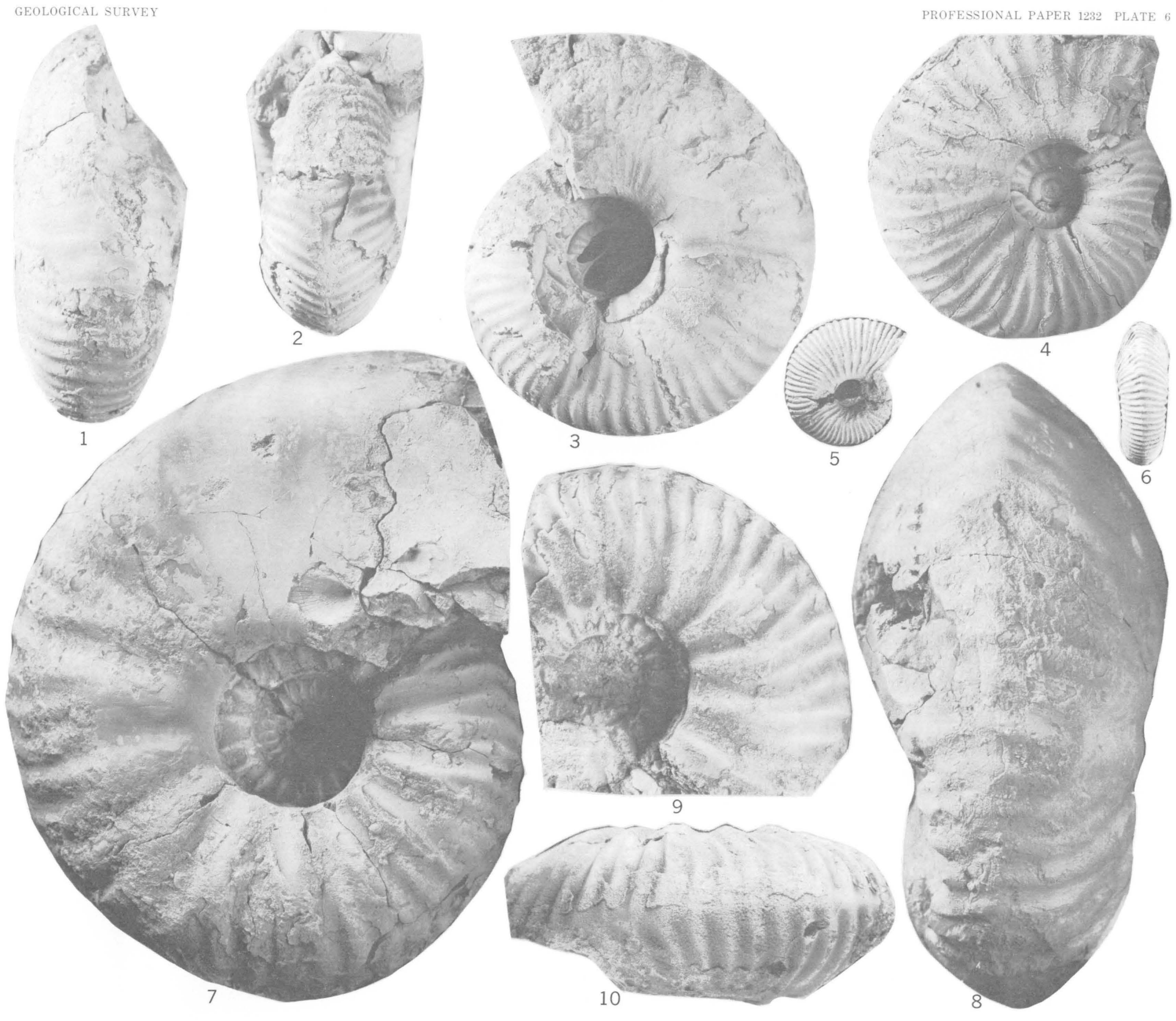

GOLIATHICERAS (GOLIATHITES) AND G. (PACHYCARDIOCERAS) 


\section{PLATE 7}

[All figures are natural size]

FIGURES 1-8. Goliathiceras (Pachycardioceras) russelli (Reeside) (p. 30)

1. Hypotype, USNM 303635, from USGS Mesozoic loc. 20519.

2. Paratype, USNM 32325, from USGS Mesozoic loc. 2388.

$3,5,7$. Ventral and lateral views of holotype, USNM 32324, from USGS Mesozoic loc. 16616.

4, 6. Lateral and ventral views of hypotype, USNM 303636, from USGS Mesozoic loc. 25176.

8. Hypotype, USNM 303634, from USGS Mesozoic loc. 11256. 


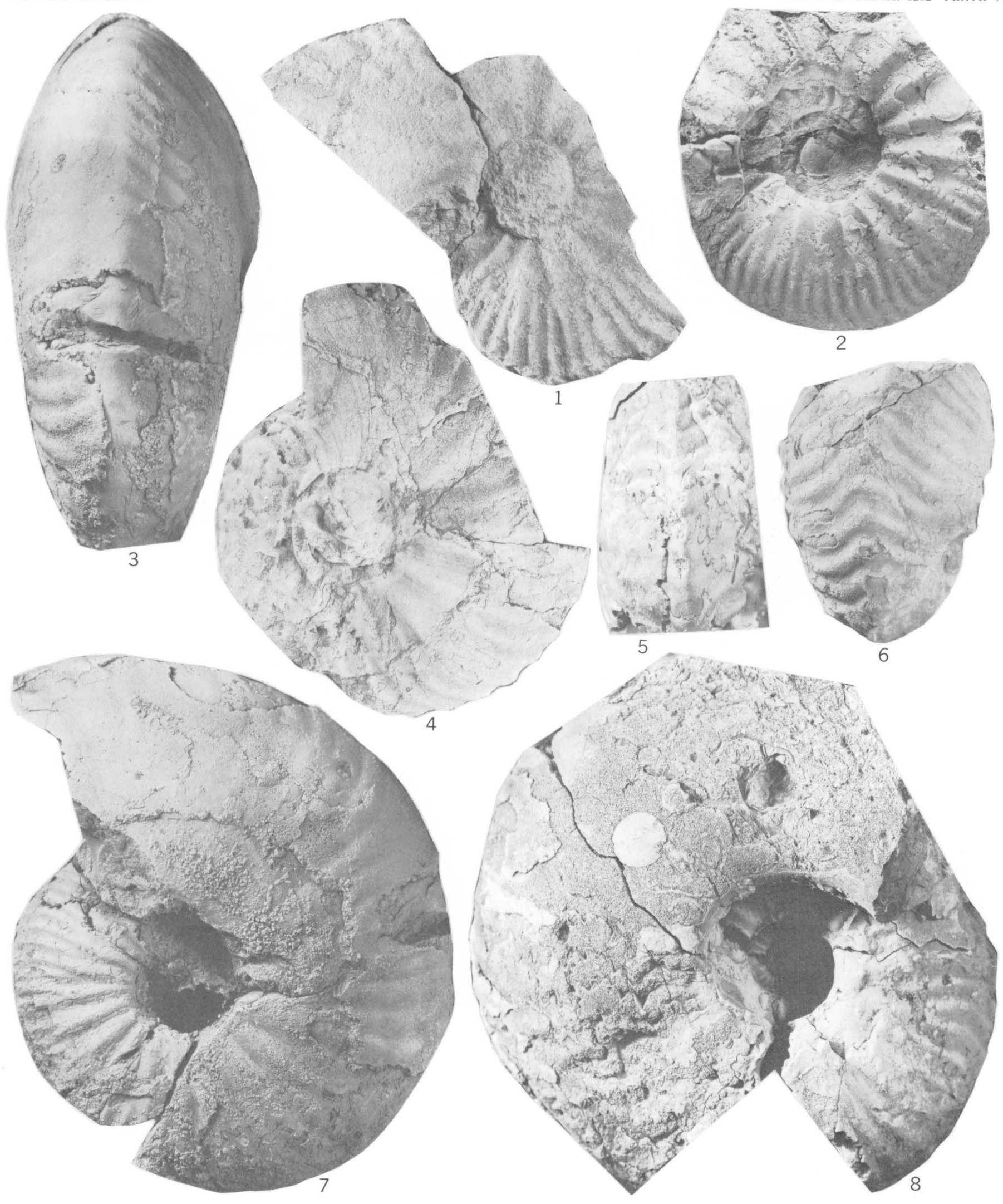

GOLIATHICERAS (PACHYCARDIOCERAS 


\section{PLATE 8}

\section{[All figures are natural size]}

FiguREs 1-4. Goliathiceras (Pachycardioceras) crassum (Reeside) (p. 30)

Hypotype, USNM 303630, from USGS Mesozoic loc. 31385. Shows parts of an inner septate whorl and an outer nonseptate whorl. 


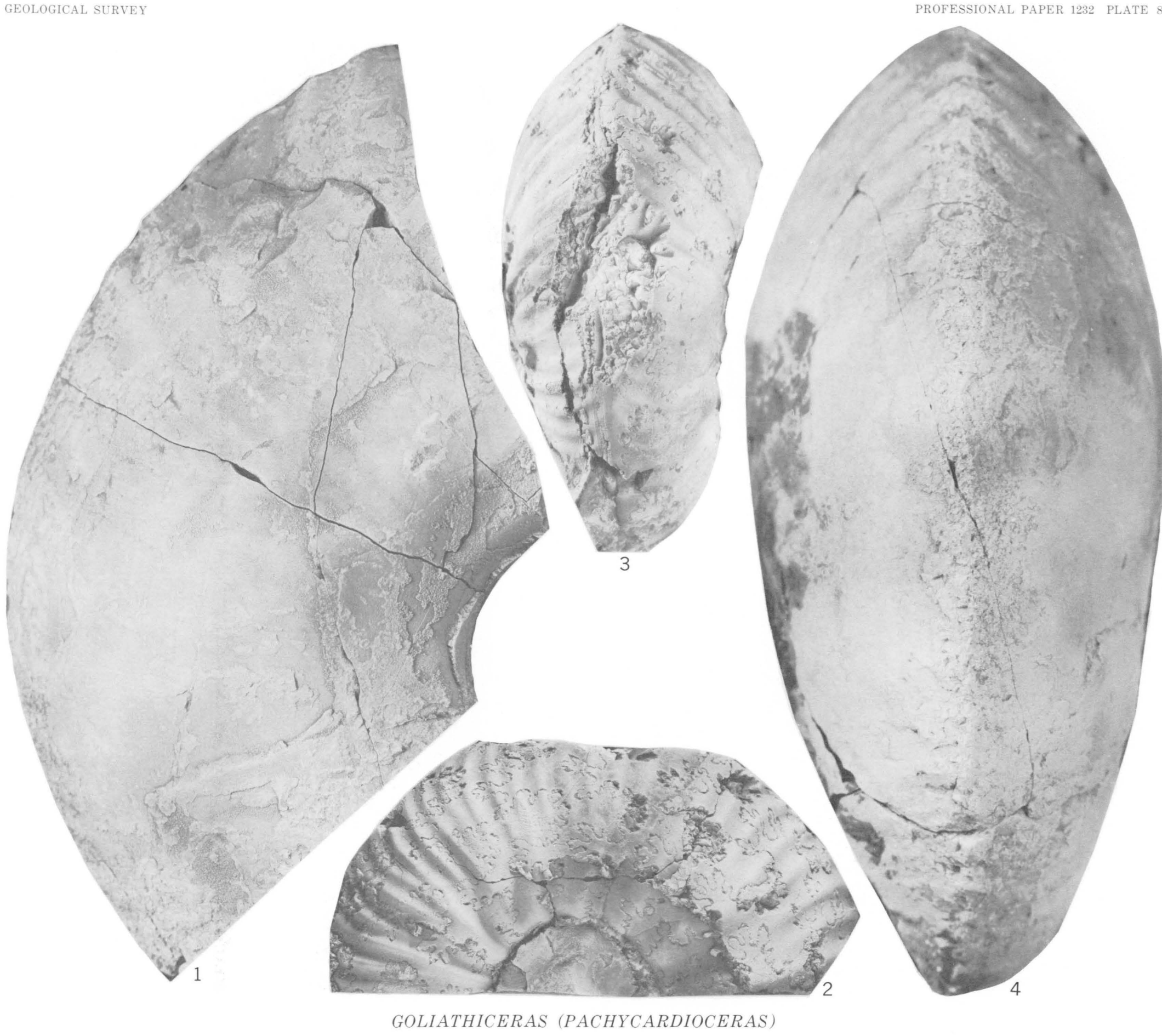




\section{PLATE 9}

[All figures are natural size]

FIGURes 1-6. Goliathiceras (Pachycardioceras) crassum (Reeside) (p. 30)

1, 2. Hypotype, USNM 29312, from USGS Mesozoic loc. 19382.

3. Hypotype, USNM 303629, from USGS Mesozoic loc. 10635.

4-6. Holotype, USNM 32323, from USGS Mesozoic loc. 2385. Figure 5 shows part of an inner whorl on the side opposite that shown in figure 6 . 


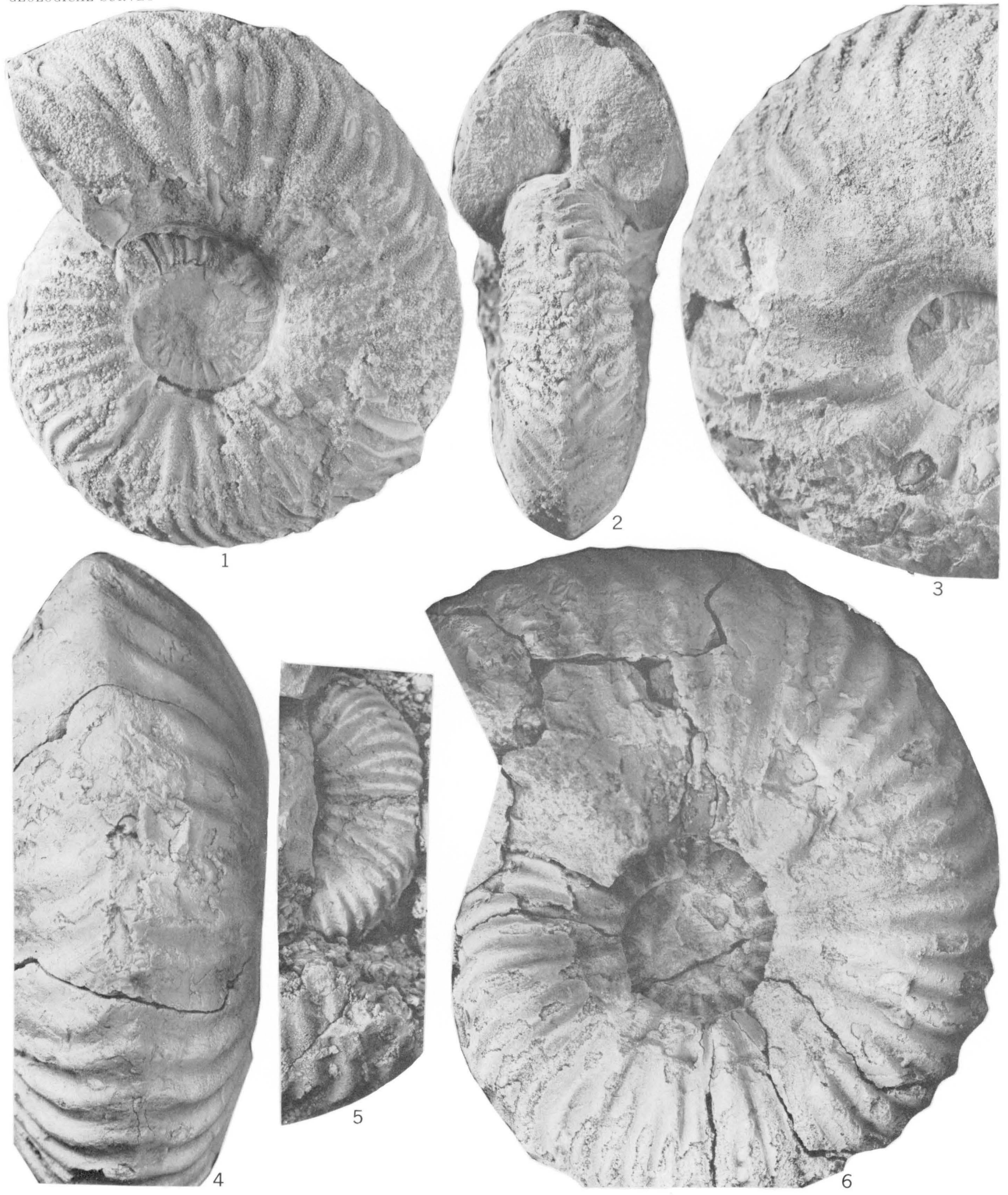

GOLIATHICERAS (PACHYCARDIOCERAS) 


\section{PLATE 10}

[Figure is slightly reduced]

FIGURE 1. Goliathiceras (Pachycardioceras) albaniense (Reeside) (p. 29)

Holotype, USNM 28448, from USGS Mesozoic loc. 2385. Shows nearly complete body chamber that occupies about three-fourths of a whorl. 


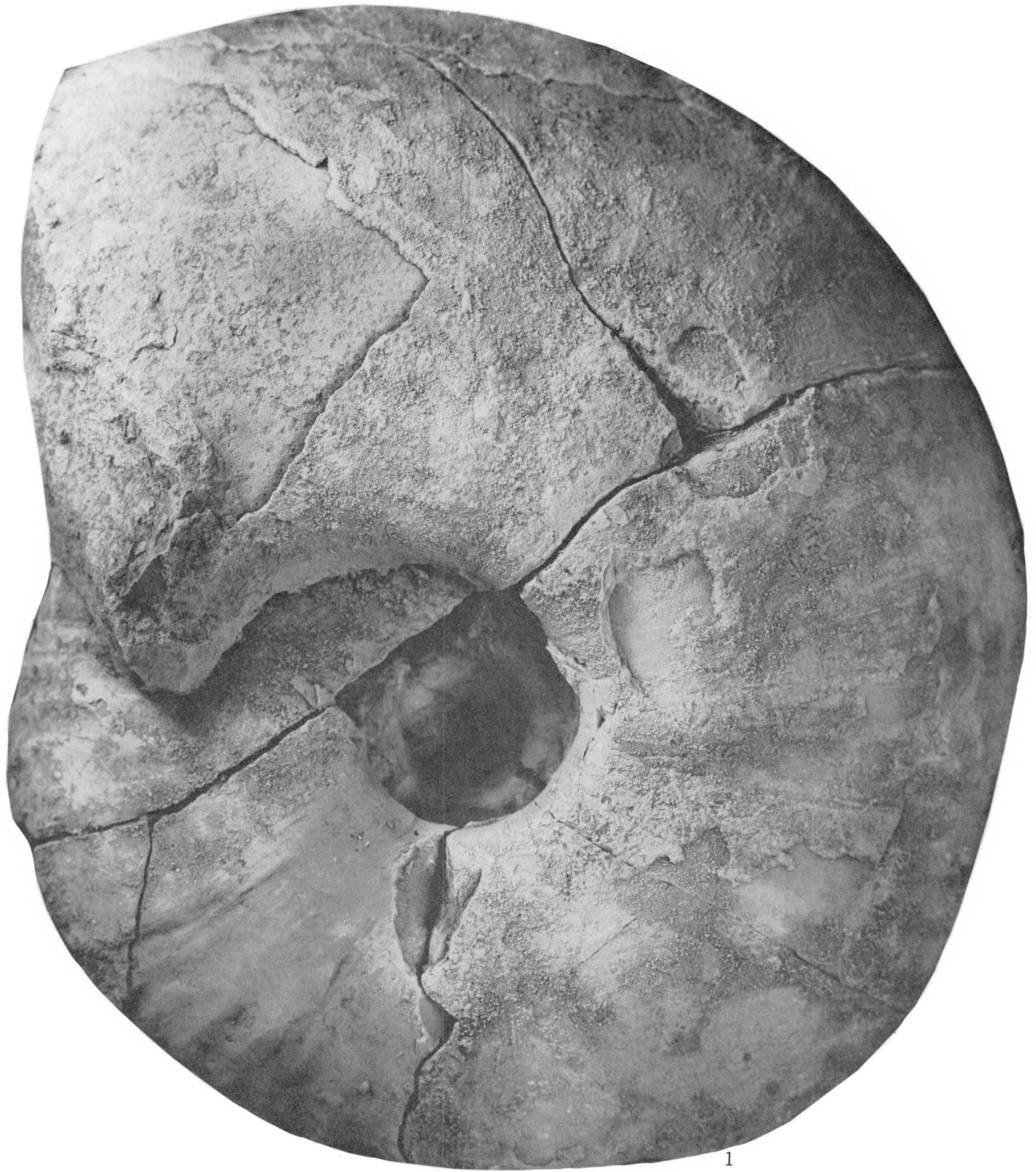

GOLIATHICERAS (PACHYCARDIOCERAS) 


\section{PLATE 11}

[Figures are slightly reduced]

FIGURES 1,2. Goliathiceras (Pachycardioceras) albaniense (Reeside) (p. 29)

Holotype, USNM 28448, from USGS Mesozoic loc. 2385. Part of body whorl has been removed to show ribbing on the outermost septate whorl. 


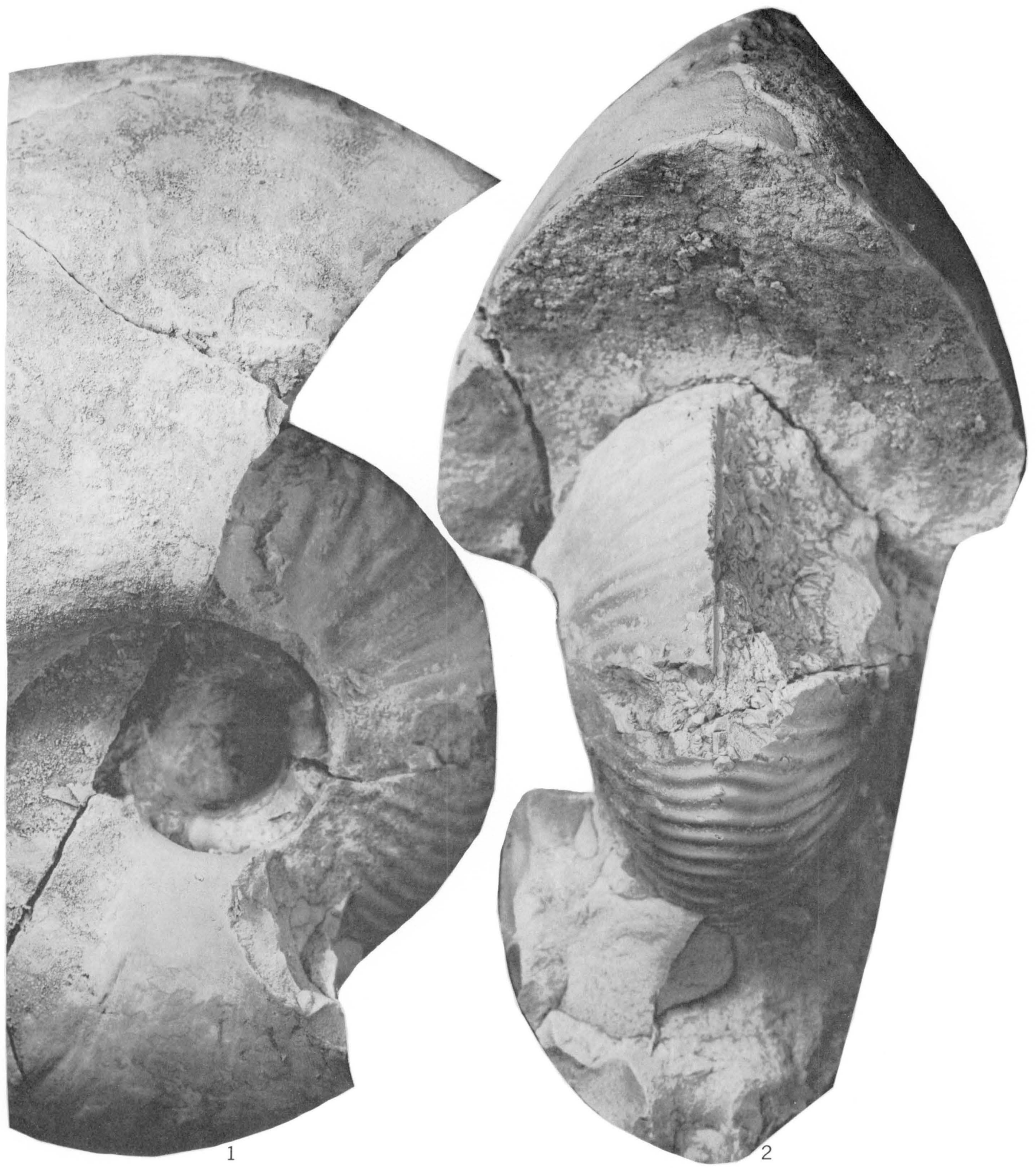

GOLIATHICERAS (PACHYCARDIOCERAS) 
PLATE 12

[Both figures are natural size]

FIGURES 1, 2. Goliathiceras (Pachycardioceras) incertum (Reeside) (p. 30)

Lateral and ventral views of paratype, USNM 32357, from USGS Mesozoic loc. 2385. 


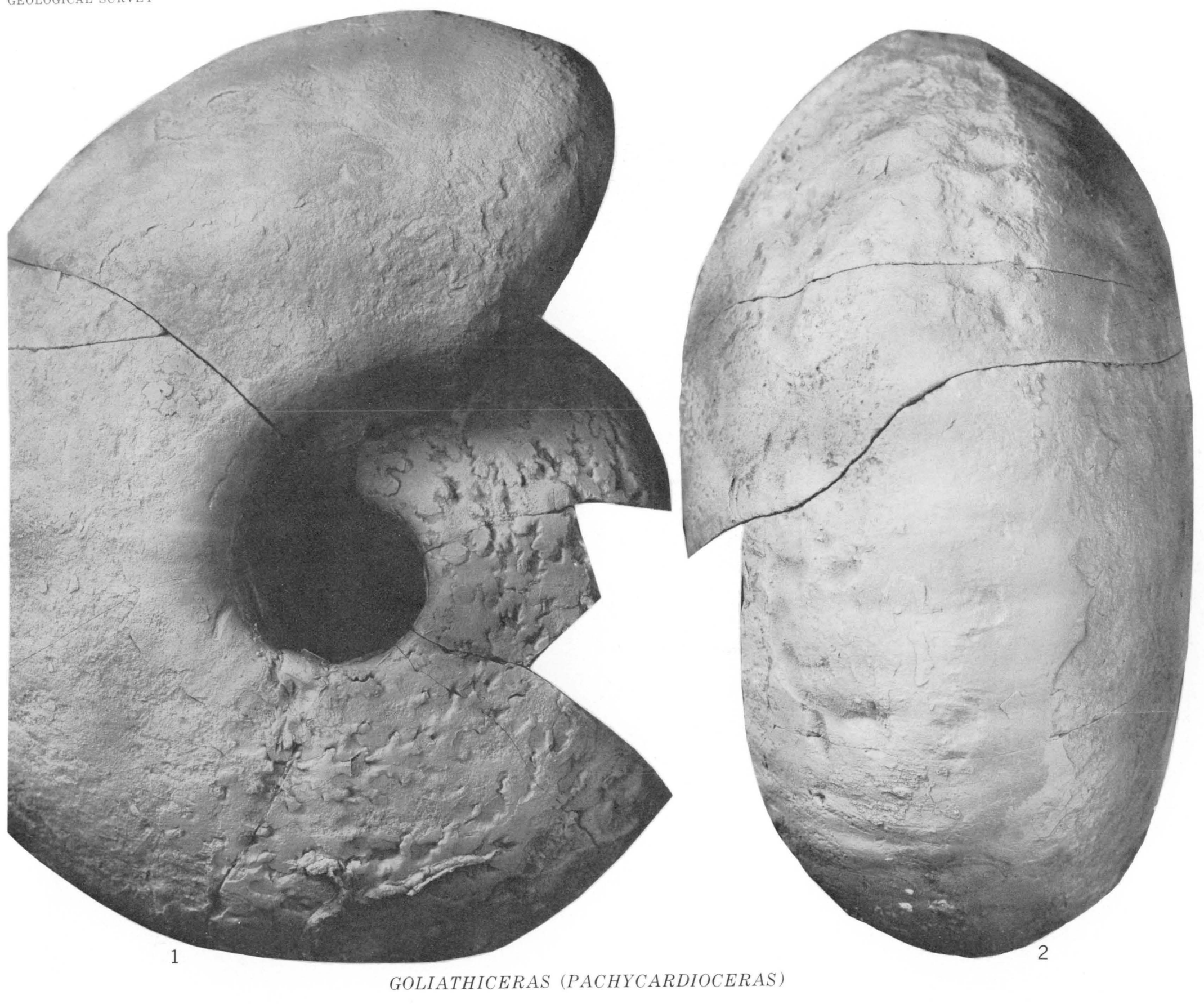




\section{PLATE 13}

[All figures are natural size]

Figures 1-5. Goliathiceras (Pachycardioceras) incertum (Reeside) (p. 30)

1, 2. Hypotype, USNM 303632, from USGS Mesozoic loc. 30109.

3-5. Holotype, USNM 32356, from USGS Mesozoic loc. 2385. Ventral and lateral views. 
GEOLOGICAL SURVEY
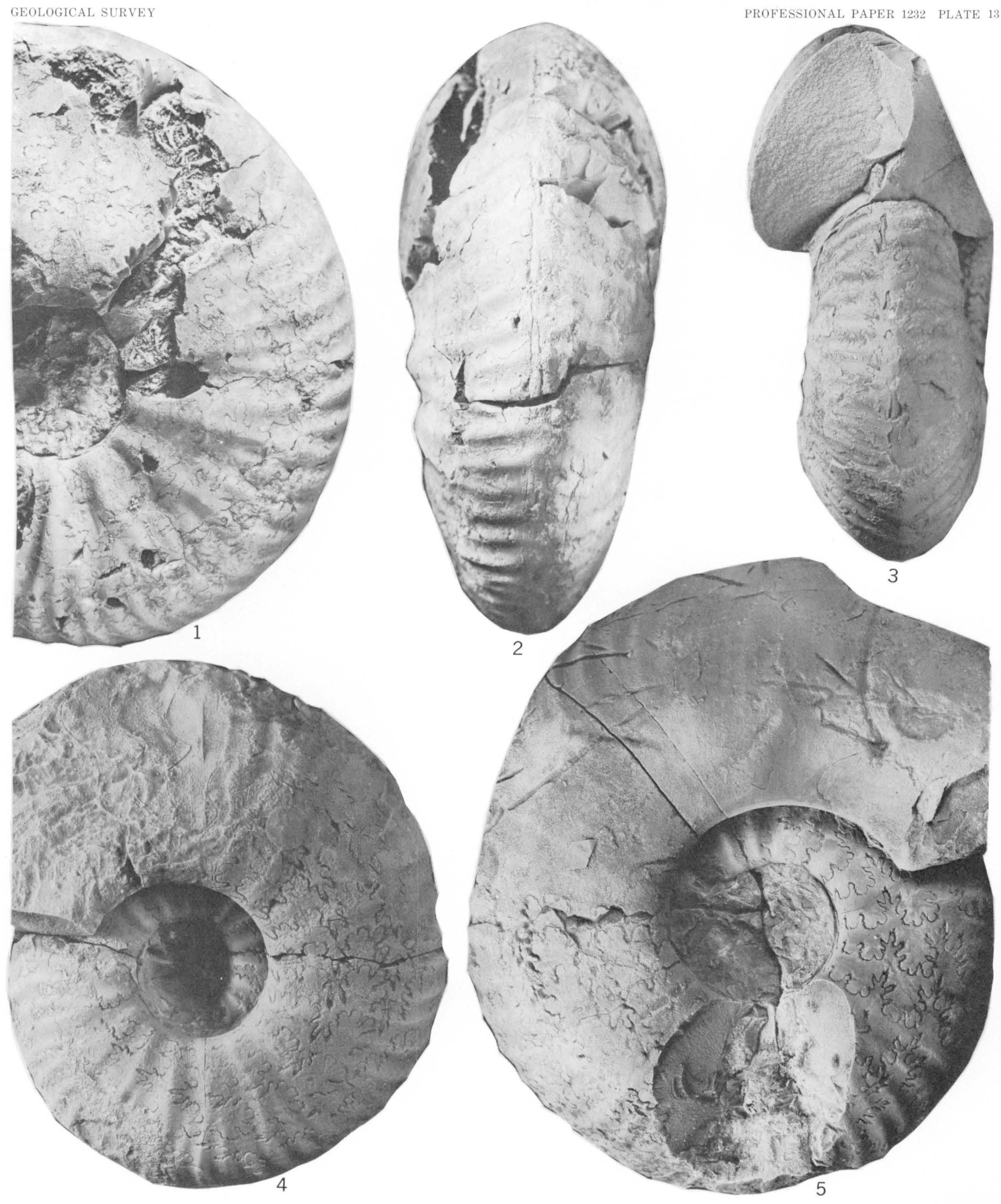

GOLIATHICERAS (PACHYCARDIOCERAS)

PROFESSIONAL PAPER 1232 PI ATE 13 


\section{PLATE 14}

[All figures are natural size]

FIGURES 1-8. Cardioceras (Scarburgiceras) crookense Reeside (p. 33)

1, 2. Apertural and lateral views of holotype, USNM 32316, from USGS Mesozoic loc. 16615

3-6. Lateral, ventral and apertural views of hypotype, USNM 303661, from USGS Mesozoic loc. 529.

7, 8. Lateral and ventral views of hypotype, USNM 303662, from USGS Mesozoic loc. 19570.

9-13. Goliathiceras (Pachycardioceras) incertum (Reeside) (p. 30)

9. Lateral view of hypotype, USNM 303633, from USGS Mesozoic loc. 28401.

10-13. Lateral and ventral views of inner septate whorl and outer nonseptate whorl of hypotype, USNM 303631, from USGS Mesozoic loc. 30109. 


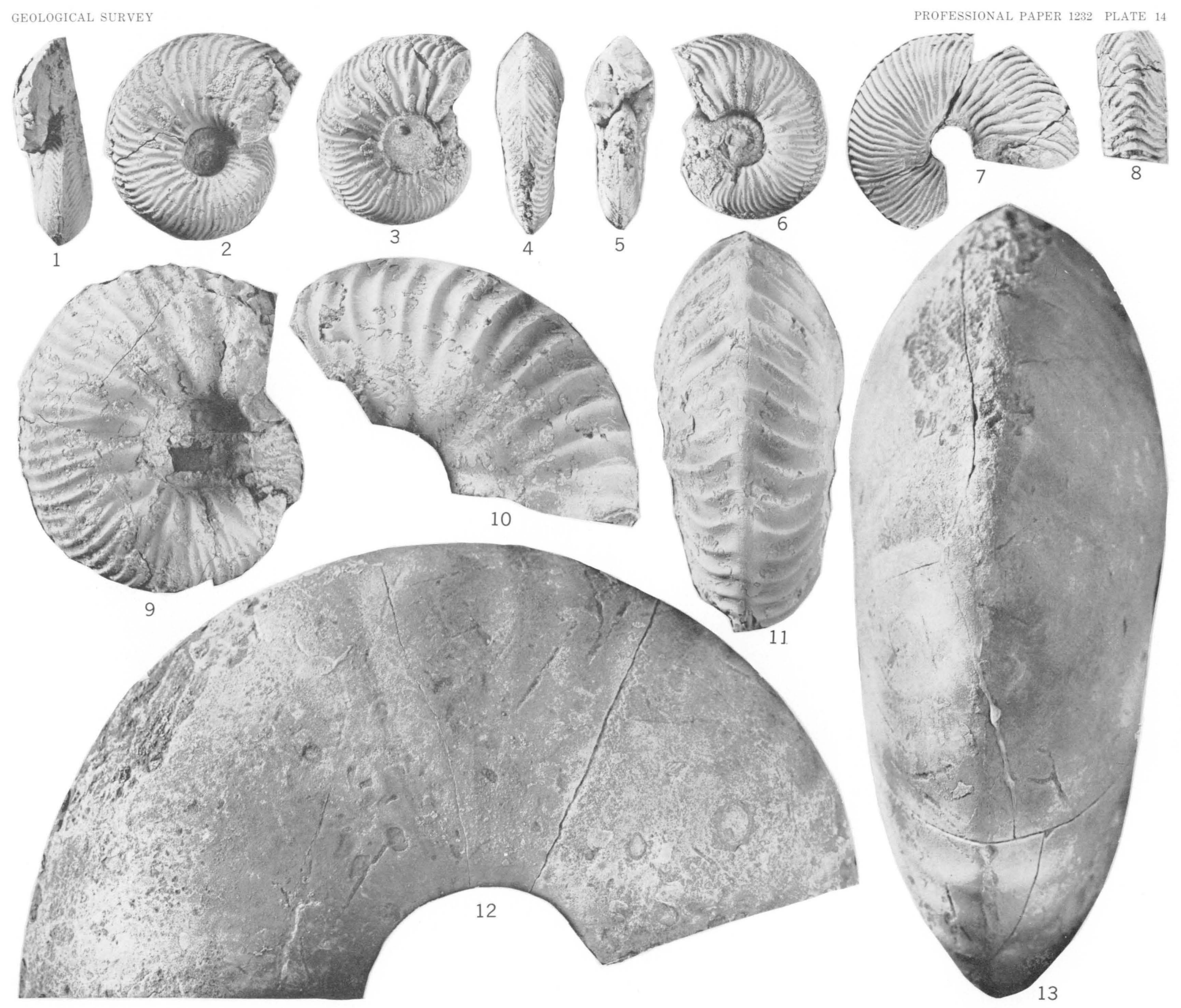

CARDIOCERAS (SCARBURGICERAS) AND GOLIATHICERAS (PACHYCARDIOCERAS) 
PLATE 15.

[All figures are natural size]

FIGURES 1-14. Cardioceras Cardioceras (Cardioceras) hyatti Reeside (p. 31)

1, 2. Holotype, USNM 32326, from USGS Mesozoic loc. 31715.

3, 4. Hypotype, USNM 303647, from USGS Mesozoic loc. 30306.

5-7. Hypotype, USNM 303648, from USGS Mesozoic loc. 24776.

8, 9. Hypotype, USNM 303650, from USGS Mesozoic loc. 24776.

10. Hypotype, USNM 303649, from USGS Mesozoic loc. 24776.

11. Hypotype, USNM 303646, from USGS Mesozoic loc. 30306.

12-14. Hypotype, USNM 303645, from USGS Mesozoic loc. 28402. Outer half whorl is nonseptate. 

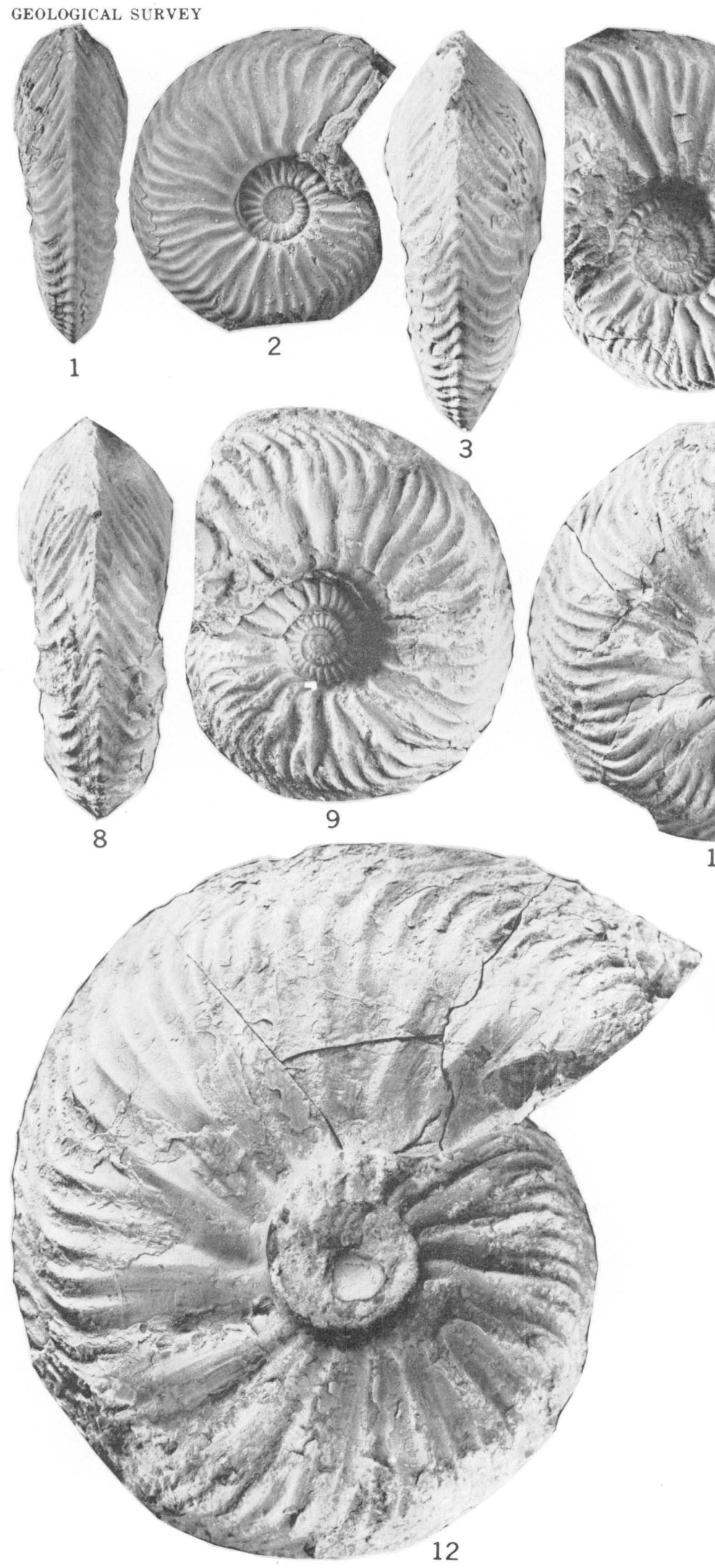

CARDIOCERAS (CARDIOCERAS
PROFESSIONAL PAPER 1232 PLATE 15
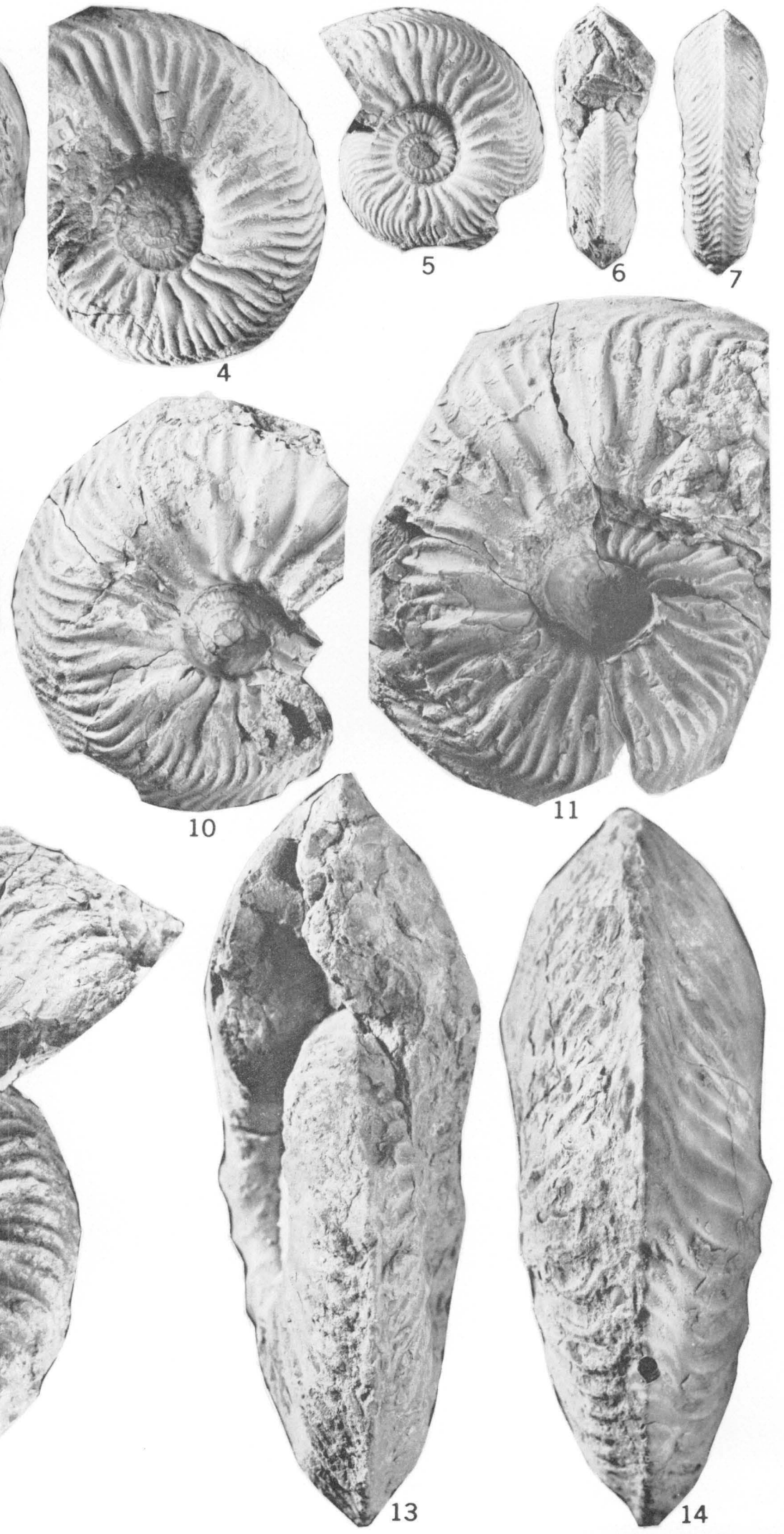


\section{PLATE 16}

[Figures natural size unless otherwise indicated]

FIGURES 1-8. Cardioceras (Scarburgiceras) reesidei Maire (p. 36).

1, 2. Hypotype, USNM 303677, from USGS Mesozoic loc. 16616.

3. Hypotype, USNM 32314, from USGS Mesozoic loc. 16616. Illustrated by Reeside (1919, pl. 8, fig. 6) as Cardioceras cordiforme (Meek and Hayden).

4, 5. Holotype, USNM 32313, from USGS Mesozoic loc. 16616. Illustrated by Reeside (1919, pl. 8, figs. 4,5) as Cardioceras cordiforme (Meek and Hayden).

6-8. Hypotype, USNM 303723, from USGS Mesozoic loc. 16616. Figures 7 and $8(\times 2)$.

9-15. Cardioceras (Cardioceras) auroraense Reeside (p. 31)

9. Hypotype, USNM 303641, from USGS Mesozoic loc. 10635.

10. Hypotype, USNM 303640, from USGS Mesozoic loc. 10635.

11-12. Hypotype, USNM 303639, from USGS Mesozoic loc. 29529.

13. Hypotype, USNM 303638, from USGS Mesozoic loc. 19567.

14. Hypotype, USNM 303637, from USGS Mesozoic loc. 25176.

15. Holotype, USNM 32319, from USGS Mesozoic loc. 2388. 

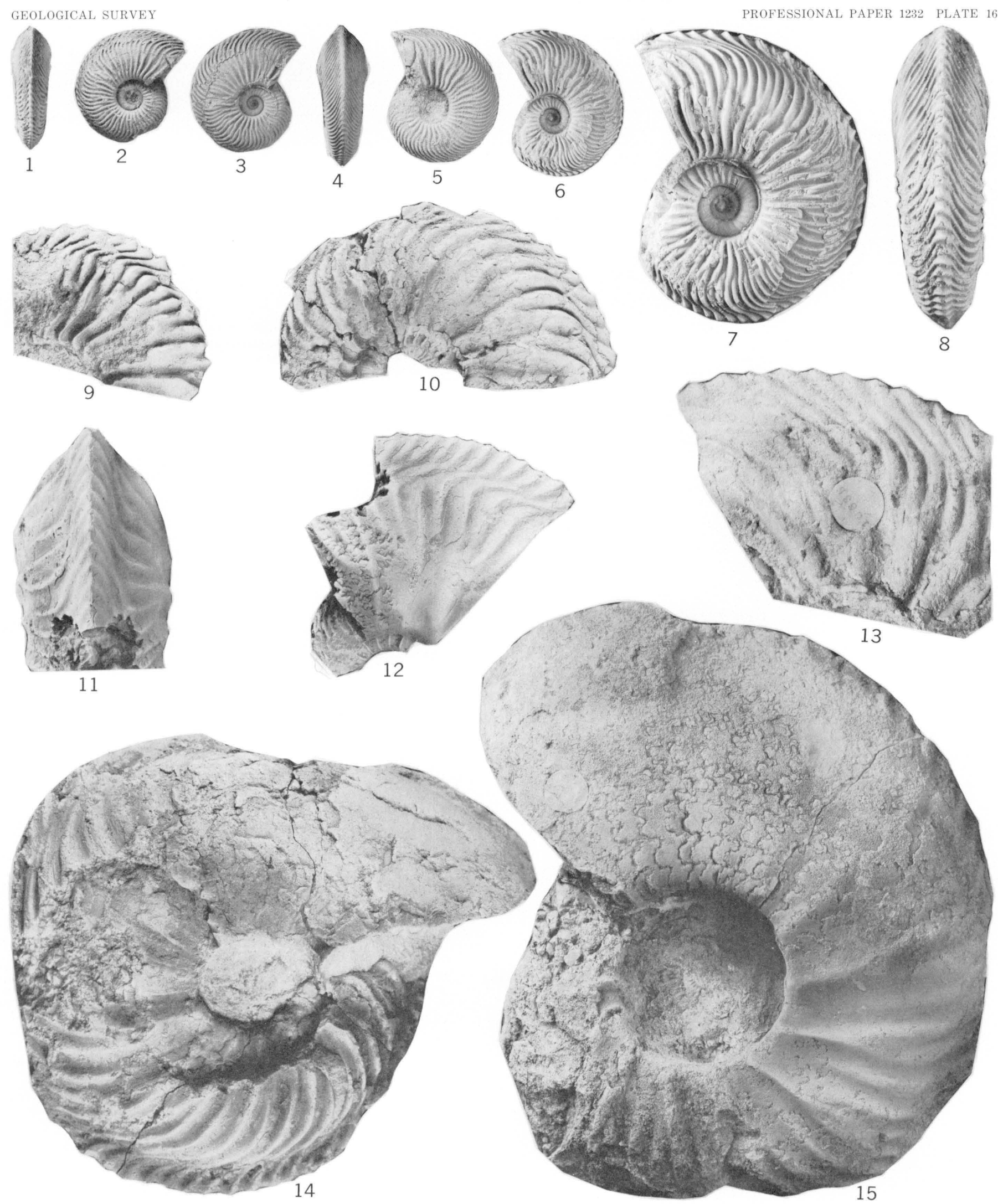

CARDIOCERAS (SCARBURGICERAS) AND C. (CARDIOCERAS) 


\section{PLATE 17}

[All figures are natural size]

FIGURES 1-16. Cardioceras (Cardioceras) distans (Whitfield) (p. 31)

1, 2. Paratype, USNM 32339, from USGS Mesozoic loc. 16620.

3. Hypotype, USNM 303644, from USGS Mesozoic loc. 31744.

4-5. Holotype, USNM 32332, from USGS Mesozoic loc. 19382.

6, 7. Paratype, USNM 12308, from USGS Mesozoic loc. 31716.

8,9. Paratype, USNM 32337, from USGS Mesozoic loc. 16620.

10, 14. Hypotype, USNM 303642, from USGS Mesozoic loc. 19567.

11. Hypotype, USNM 303643, from USGS Mesozoic loc. 31744.

12. Paratype, USNM 32334, from USGS Mesozoic loc. 19382.

13, 15, 16. Paratype, USNM 32333, from USGS Mesozoic loc. 19382. 

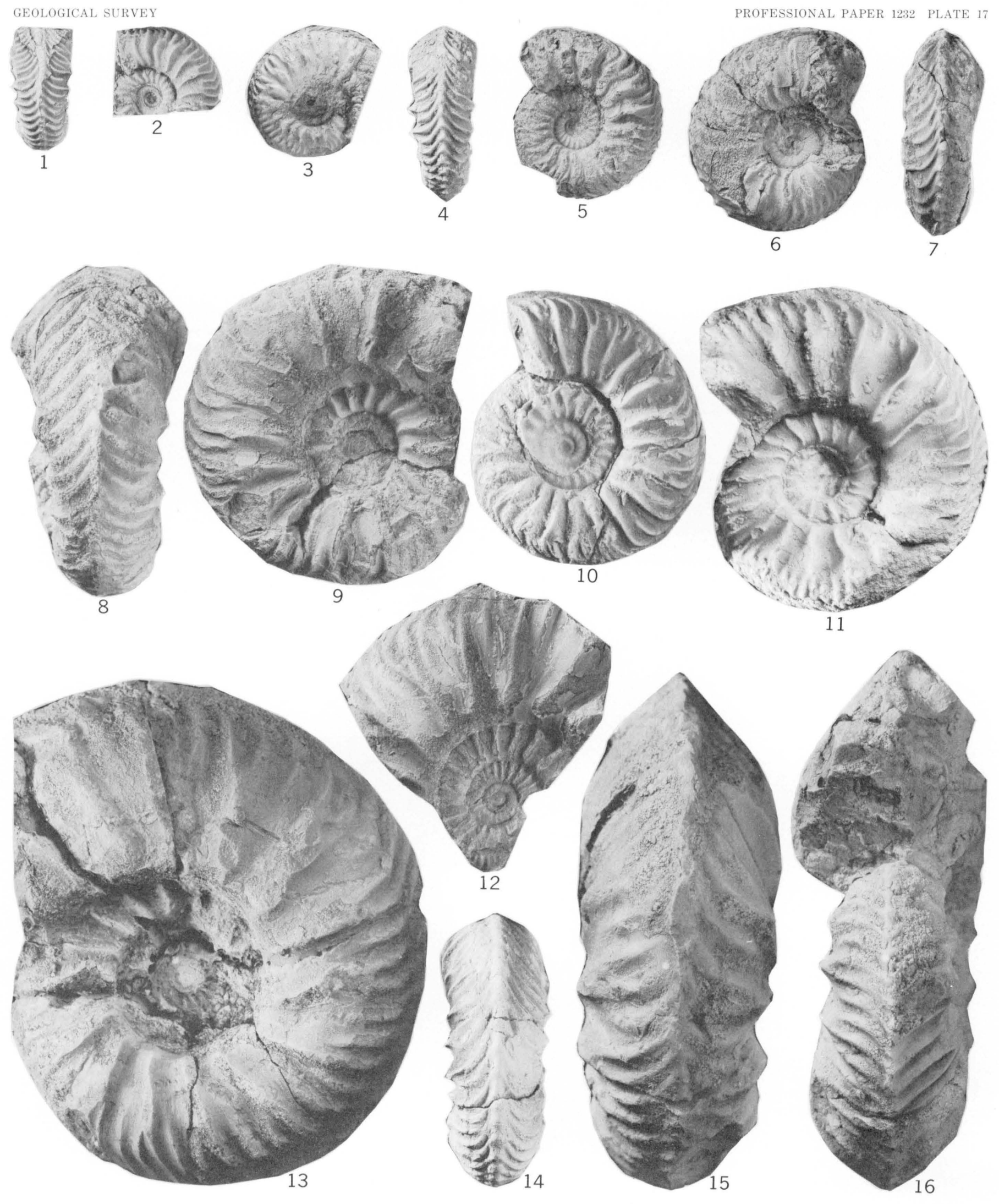

CARDIOCERAS (CARDIOCERAS) 


\section{PLATE 18}

[All figures natural size unless otherwise indicated]

FIGURES 1-8. Cardioceras (Scarburgiceras) schucherti Reeside (p. 34)

1, 2. Hypotype, USNM 303669, from USGS Mesozoic loc. $31382(\times 2)$.

3. Hypotype, USNM 303666, from USGS Mesozoic loc. 30911. Photo based on rubber imprint of an external mold.

4, 5. Hypotype, USNM 303668, from USGS Mesozoic loc. 19205.

6. Hypotype, USNM 303667, from USGS Mesozoic loc. 24735.

7, 8. Holotype, USNM 32322, from USGS Mesozoic loc. 2385.

9-21. Cardioceras (Scarburgiceras) americanum Reeside (p. 32)

9, 10. Hypotype, USNM 303652, from USGS Mesozoic loc. 19204.

11, 12. Paratype, USNM 32309, from USGS Mesozoic loc. 16620.

13-15. Holotype, USNM 32308, from USGS Mesozoic loc. 16620.

16, 19-21. Hypotype, USNM 303651, from USGS Mesozoic loc. 29529. Inner whorl attaches to lower part of adoral end of specimen shown on figure 19.

17, 18. Hypotype, USNM 303653, from USGS Mesozoic loc. $19207(\times 2)$. 


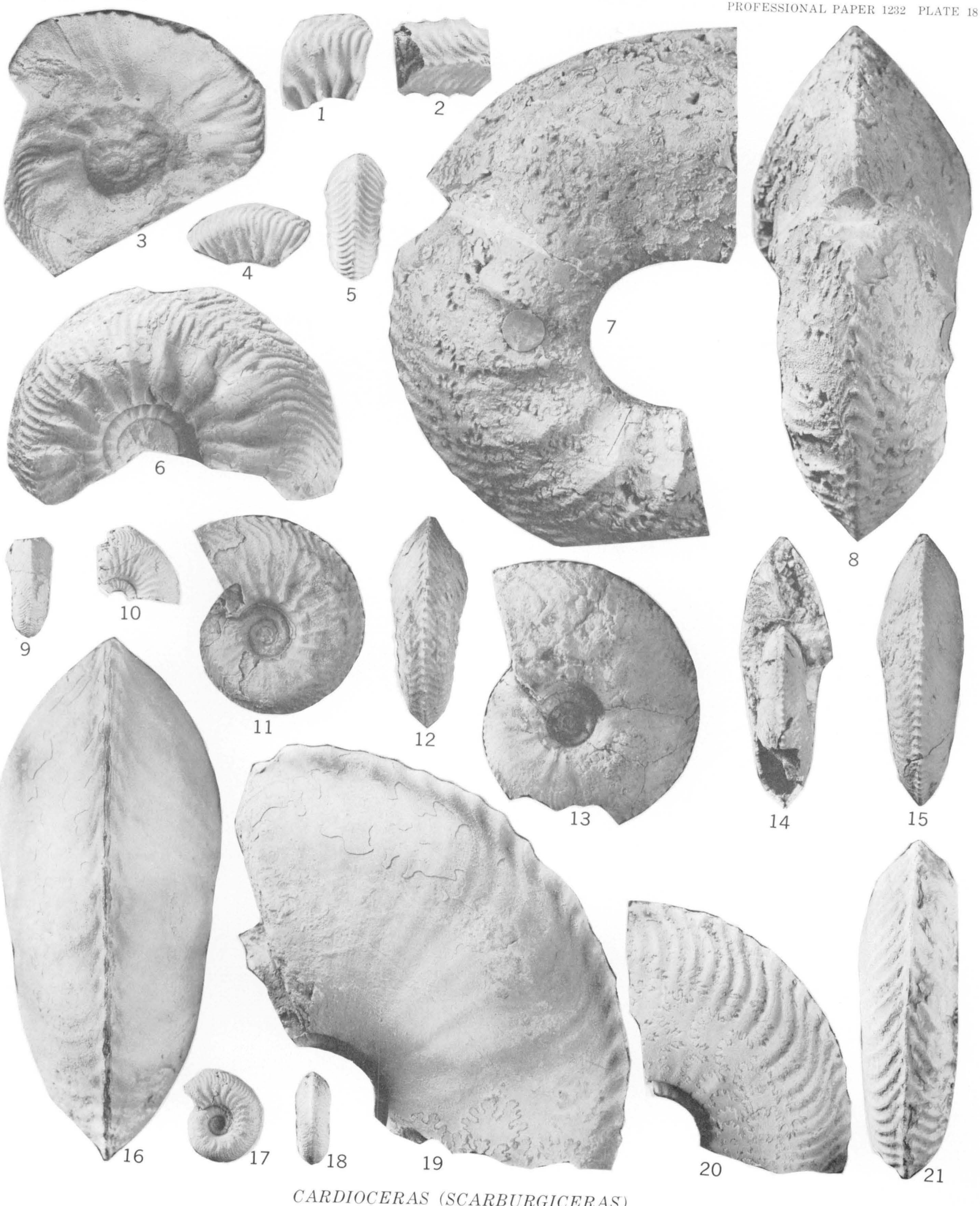




\section{PLATE 19}

[All figures are natural size]

FIGURES 1-7. Cardioceras (Scarburgiceras?) mathiaspeakense Imlay, n. sp. (p. 34)

1, 2. Paratype, USNM 303664, from USGS Mesozoic loc. 19568.

3, 4. Paratype, USNM 303665, from USGS Mesozoic loc. 25176.

5-7. Holotype, USNM 303663, from USGS Mesozoic loc. 25176. Body chamber represents about half of a whorl. 

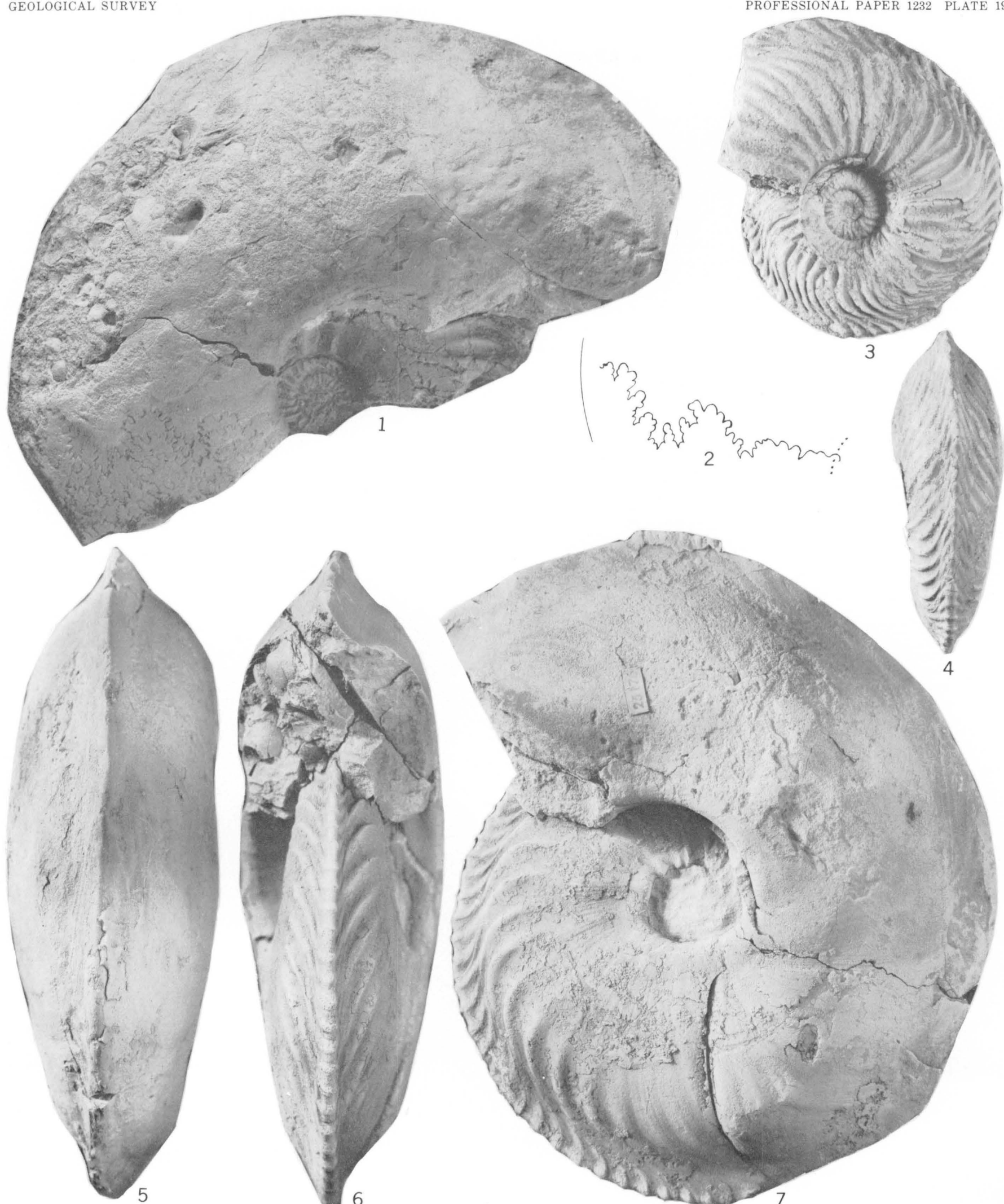

32

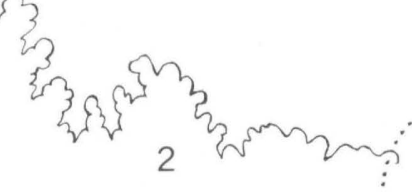

3
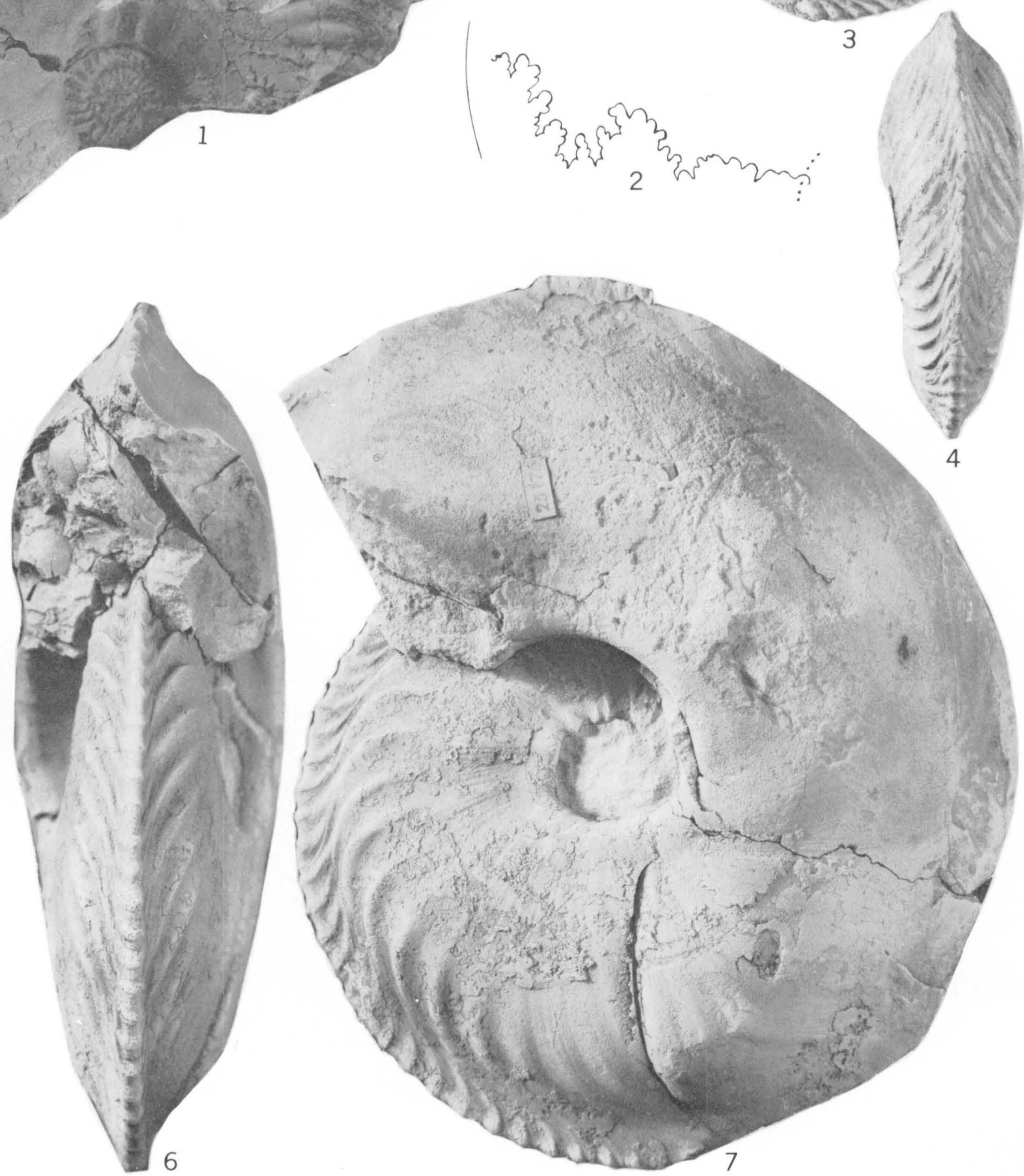

CARDIOCERAS (SCARBURGICERAS?) 


\section{PLATE 20}

[All figures are natural size]

Figures 1-7. Cardioceras (Scarburgiceras) bighornense Imlay, n. sp. (p. 32)

1, 3. Apertural and lateral views of paratype, USNM 303657, from USGS Mesozoic loc. 29770.

2. Rubber imprint of external mold of paratype, USNM 303655, from USGS Mesozoic loc. 29751

4, 5. Paratype, USNM 303656, from USGS Mesozoic loc. 26717.

6, 7. Holotype, USNM 303654, from USGS Mesozoic loc. 29770. 

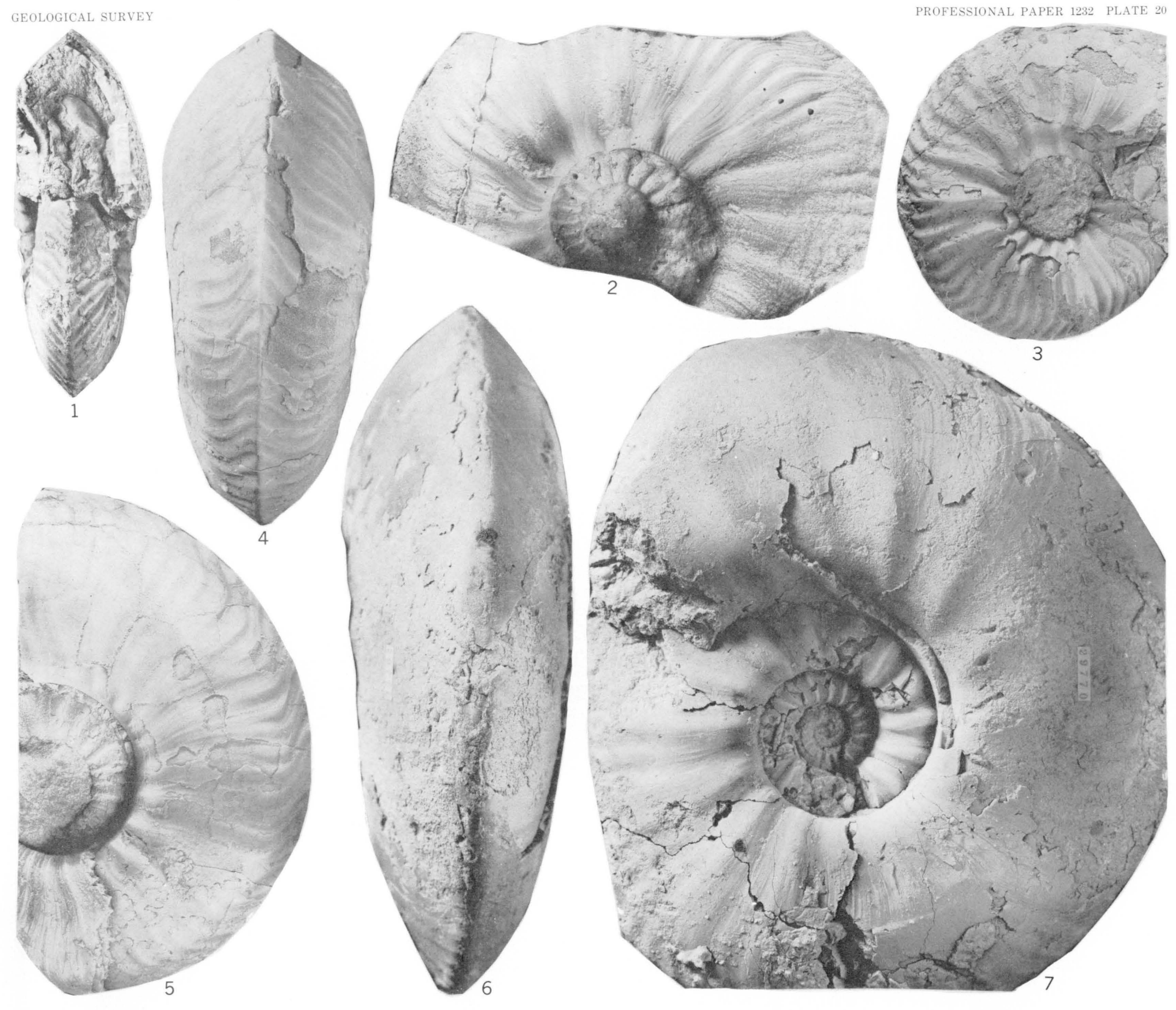

CARDIOCERAS (SCARBURGICERAS) 


\title{
PLATE 21
}

\author{
[All figures are natural size]
}

FIGURES 1-15. Cardioceras (Scarburgiceras) wyomingense Reeside (p. 35)

1, 2. Paratype, USNM 32331, from USGS Mesozoic loc. 16620.

3. Hypotype, USNM 303676, from USGS Mesozoic loc. 29534. 4, 5. Holotype, USNM 32329, from USGS Mesozoic loc. 529.

6, 7. Hypotype, USNM 303675, from USGS Mesozoic loc. 29528.

8. Hypotype, USNM 303674, from USGS Mesozoic loc. 29528.

9, 10. Hypotype, USNM 303672, from USGS Mesozoic loc. 29528.

11, 13. Hypotype, USNM 303673, from USGS Mesozoic loc. 30324.

12. Hypotype, USNM 303671, from USGS Mesozoic loc. 29534. 14, 15. Hypotype, USNM 303670, from USGS Mesozoic loc. 19171. 

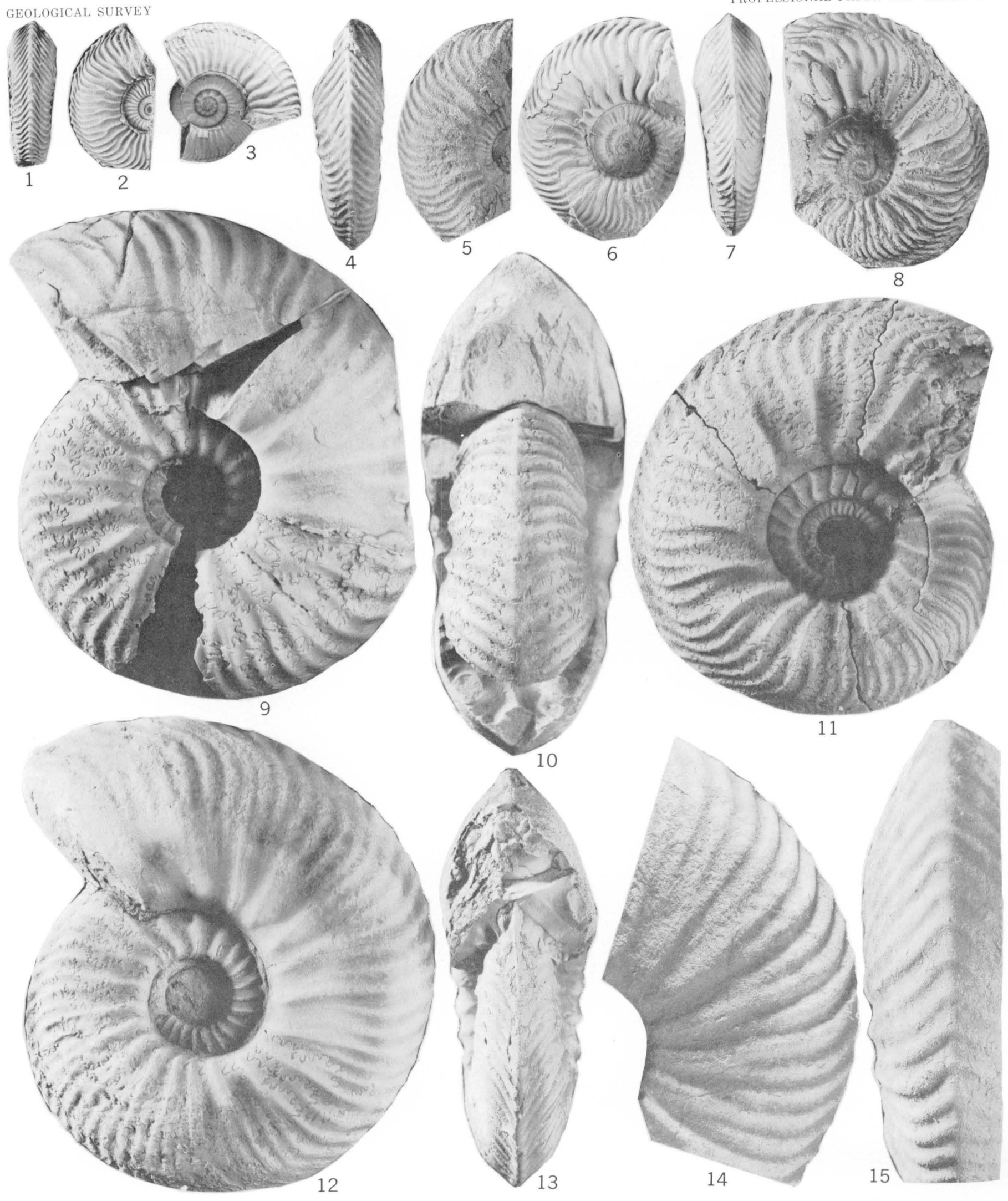

CARDIOCERAS (SCARBURGICERAS) 


\section{PLATE 22}

[All figures natural size unless otherwise indicated]

FIGURES 1-3, 8-10. Cardioceras (Scoticardioceras) whitfieldi Reeside (p. 36)

1-3. Paratype, USNM 12345, from USGS Mesozoic loc. 31716.

8-10. Holotype, USNM 12294, from USGS Mesozoic loc. 31716.

11-13. Cardioceras (Scoticardioceras) stillwelli Reeside (p. 36)

Holotype, USNM 32307, from USGS Mesozoic loc. 16620.

4. Cardioceras (Scoticardioceras)? cf. C. (S.) whitfieldi Reeside (p. 36).

Rubber imprint of figured specimen, USNM 303679, from USGS Mesozoic loc. 27641.

5-7. Cardioceras (Scoticardioceras) cf. C. (S.) stillwelli Reeside (p. 36).

Figured specimen, USNM 303678, from USGS Mesozoic loc. 19579. Figure $5(\times 2)$.

14-21, 26-28. Cardioceras (Cawtoniceras) whiteavesi Reeside (p. 37)

14, 15. Hypotype, USNM 303684, from USGS Mesozoic loc. 30102.

16, 17. Hypotype, USNM 303686, from USGS Mesozoic loc. 31720.

18, 19. Hypotype, USNM 303685, from USGS Mesozoic loc. 30102.

0, 21. Hypotype, USNM 303683, from USGS Mesozoic loc. 28400.

26-28. Holotype, USNM 32340, from USGS Mesozoic loc. 16620.

22-25, 29-32. Cardioceras (Scarburgiceras) cordiforme (Meek and Hayden) (p. 33)

22, 24, 25. Hypotype, USNM 303659, from USGS Mesozoic loc. 11256.

23. Hypotype, USNM 303660, from USGS Mesozoic loc. 11256.

29, 30. Hypotype, USNM 303658, from USGS Mesozoic loc. 29541.

31, 32. Holotype (septate whorls only), USNM 203, USGS collections from southwest base of Black Hills, South Dakota. 


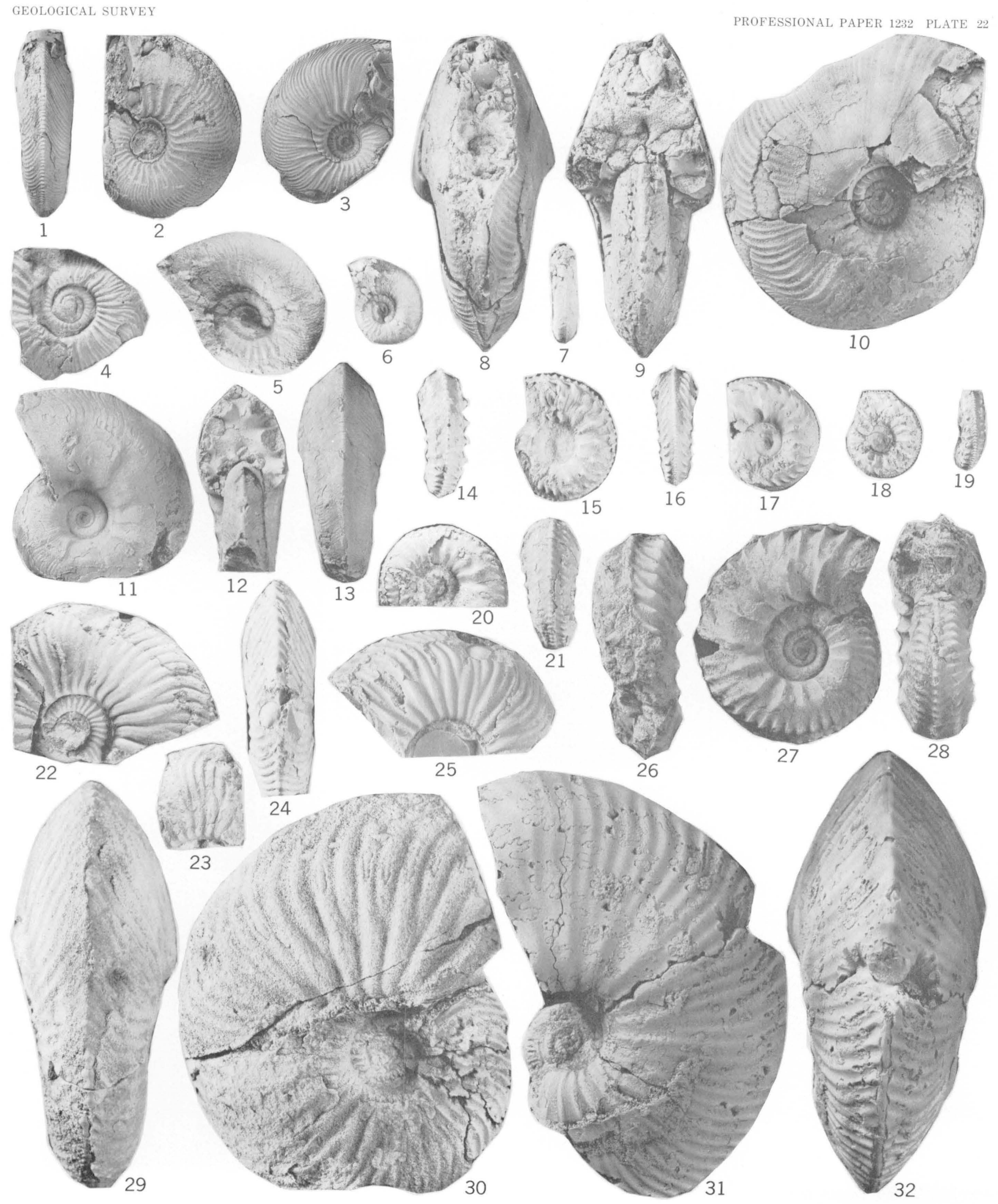

CARDIOCERAS (SCOTICARDIOCERAS), C. (CAWTONICERAS), AND C. (SCARBURGICERAS) 


\section{PLATE 23}

[All figures are natural size]

FIGURES 1-16. Cardioceras (Maltoniceras) sundancense Reeside (p. 37)

1-4. Ventral, apertural and lateral views of hypotype, USNM 303691, from USGS Mesozoic loc. 31754.

5. Rubber imprint of hypotype, USNM 303690, from USGS Mesozoic loc. 29278.

6. Hypotype, USNM 303689, from USGS Mesozoic loc. 29278.

7-9. Paratype, USNM 32346, from USGS Mesozoic loc. 16620.

10. Hypotype, USNM 303688, from USGS Mesozoic loc. 19612.

11, 14. Paratype, USNM 32345, from USGS Mesozoic loc. 16620.

12, 13. Paratype, USNM 32344, from USGS Mesozoic loc. 16620.

15. Hypotype, USNM 303687, from USGS Mesozoic loc. 30087.

16. Holotype, USNM 29314, from USGS Survey Mesozoic loc. 16620. 


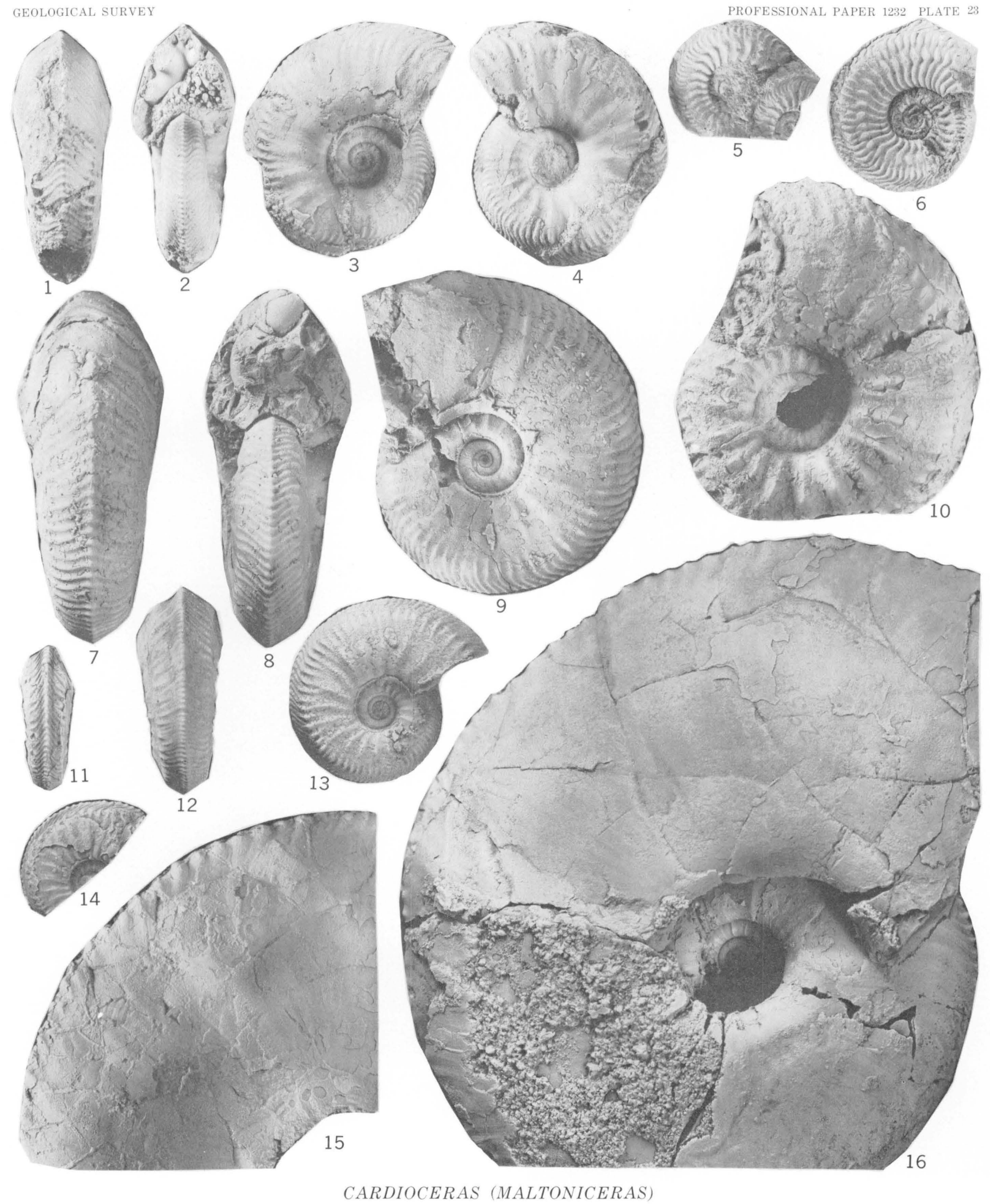




\title{
PLATE 24
}

\author{
[All figures are natural size]
}

FiguREs 1-18, 21. Cardioceras (Maltoniceras?) reddomense Imlay, n. sp. (p. 38)

1, 2. Paratype, USNM 303701, from USGS Mesozoic loc. 19612.

3, 4. Paratype, USNM 303694, from USGS Mesozoic loc. 30087.

5, 6. Paratype, USNM 303700, from USGS Mesozoic loc. 19612.

7, 8. Paratype, USNM 303698, from USGS Mesozoic loc. 29769.

9, 10. Holotype, USNM 303692, from USGS Mesozoic loc. 30087.

11, 12. Paratype, USNM 303699, from USGS Mesozoic loc. 19612.

13, 14. Paratype, USNM 303693, from USGS Mesozoic loc. 30087.

15, 18. Paratype, USNM 303697, from USGS Mesozoic loc. 29769.

16, 17. Paratype, USNM 303696, from USGS Mesozoic loc. 29769.

21. Paratype, USNM 303695, from USGS Mesozoic loc. 29769.

19, 20. Cardioceras (Maltomiceras) plattense Reeside (p. 37)

Holotype, USNM 32318, from USGS Mesozoic loc. 2386. 


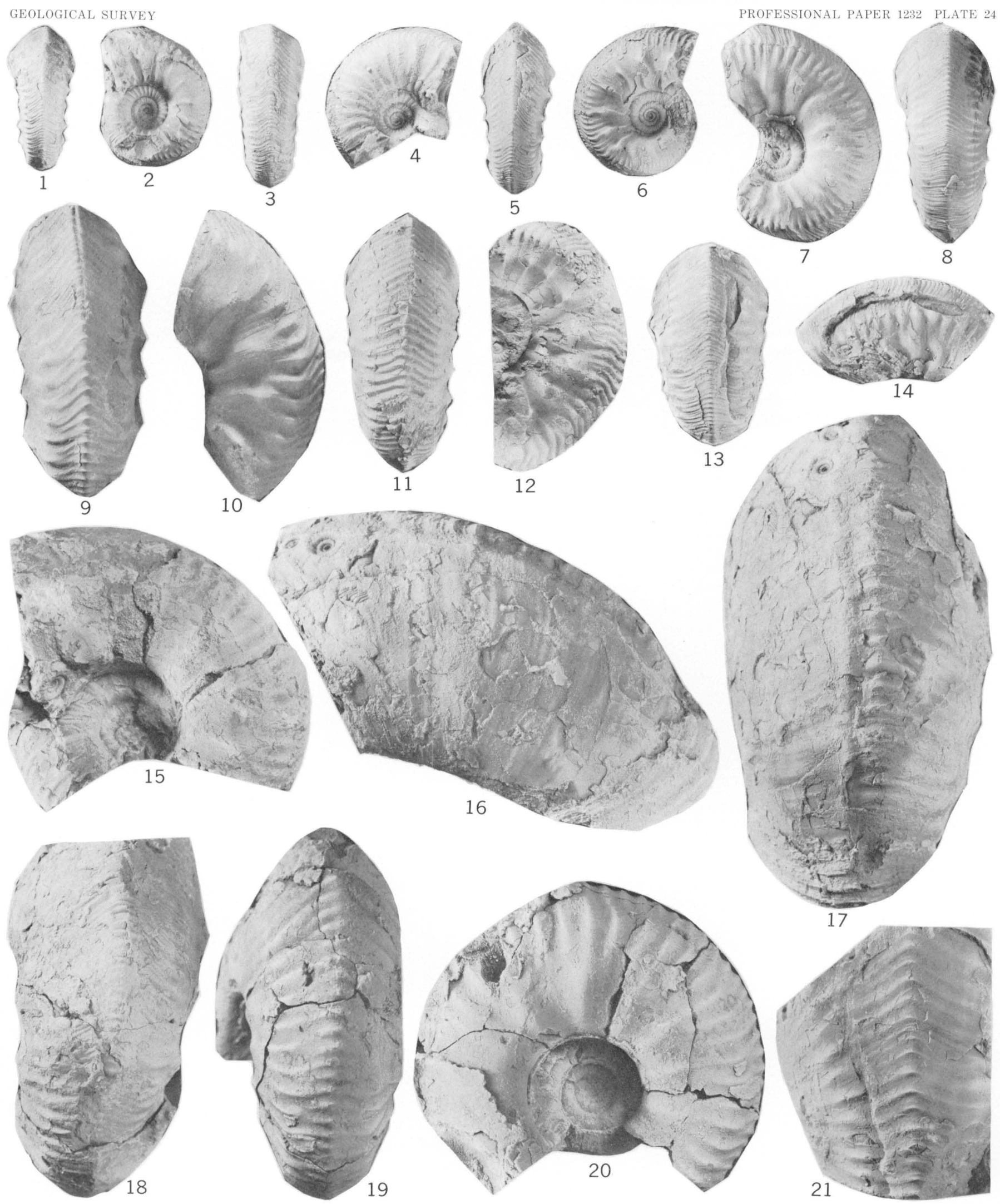

CARDIOCERAS (MALTONICERAS?) AND C. (MALTONICERAS) 


\section{PLATE 25}

[All figures natural size unless otherwise indicated]

Figures 1-10. Cardioceras (Vertebriceras) haresi Reeside (p. 39)

1, 2. Hypotype, USNM 303715, from USGS Mesozoic loc. 28816.

3, 4. Hypotype, USNM 303714, from USGS Mesozoic loc. 28816.

5, 6. Holotype, USNM 32349, from USGS Mesozoic loc. 16616.

7, 8. Hypotype, USNM 303716, from USGS Mesozoic loc. 28816.

9, 10. Paratype, USNM 32350, from USGS Mesozoic loc. 9357.

11-13. Grossouvria? sp. (p. 40)

Figured specimen, USNM 303721, from USGS Mesozoic loc. 19577. Figures 12 and $13(\times 2)$.

14, 15. Grossouvria (Poculisphinctes?) cf. G. (P.) trina (Buckman) (p. 40).

Figured specimen, USNM 303720, from USGS Mesozoic loc. 19570.

16-26. Cardioceras (Vertebriceras) stantoni Reeside (p. 39)

16-18. Hypotype, USNM 303713, from USGS Mesozoic loc. 19571.

19. Hypotype, USNM 303712, from USGS Mesozoic loc. 19571.

20, 21. Paratype, USNM 32328, from USGS Mesozoic loc. 31715.

22-24. Hypotype, USNM 303711, from USGS Mesozoic loc. 19571.

25, 26. Hypotype, USNM 303710, from USGS Mesozoic loc. 19571. 

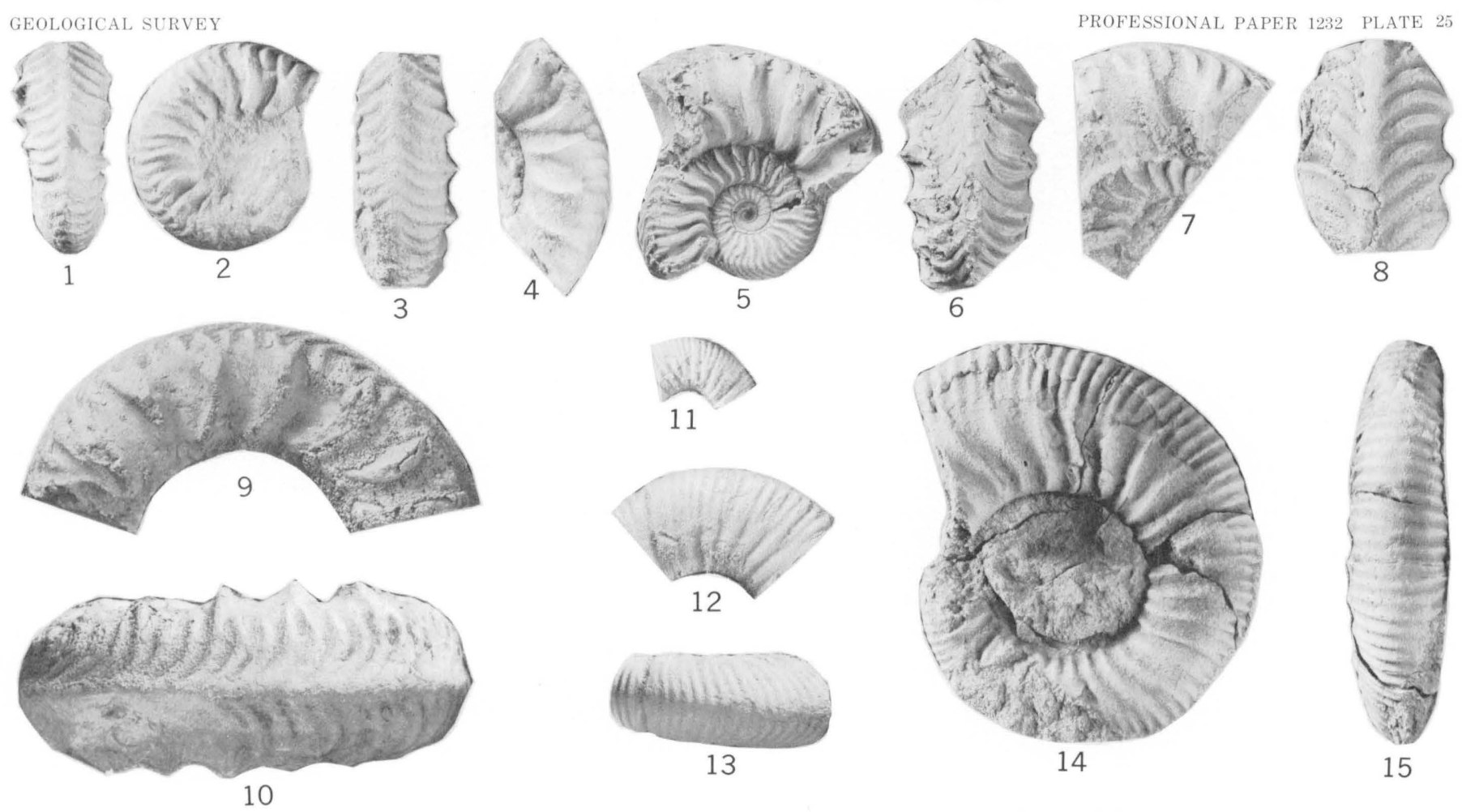

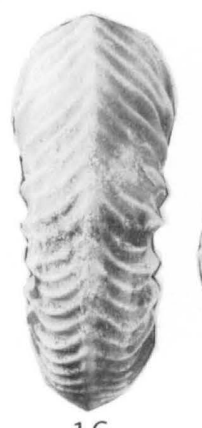

16
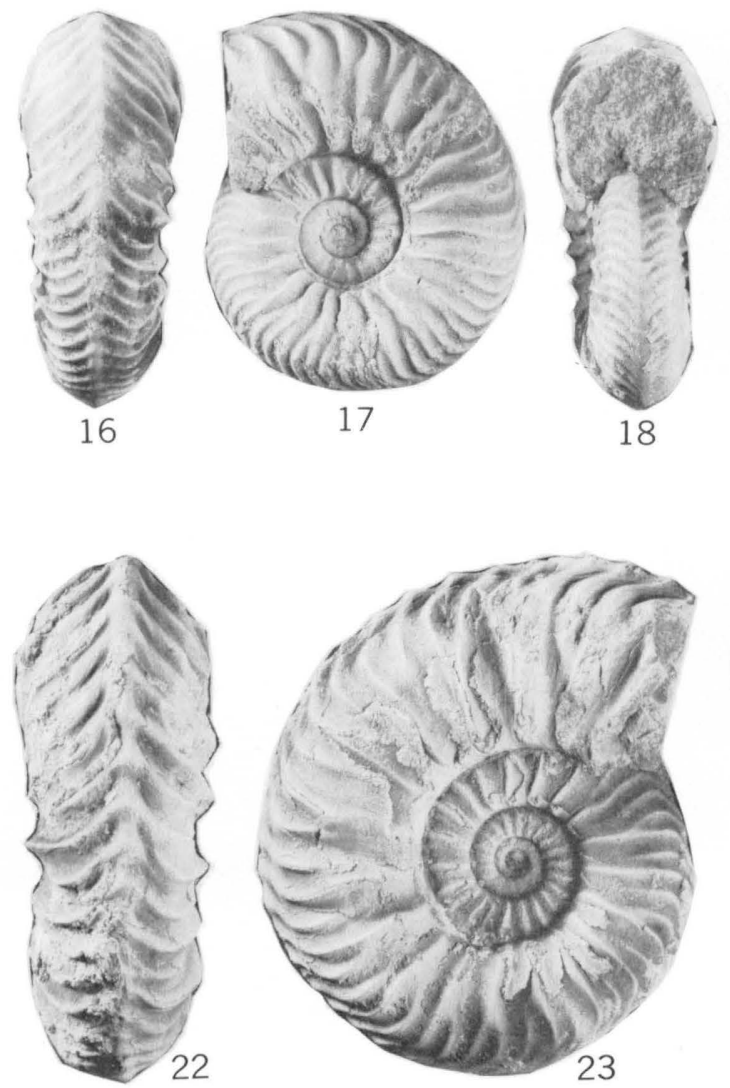
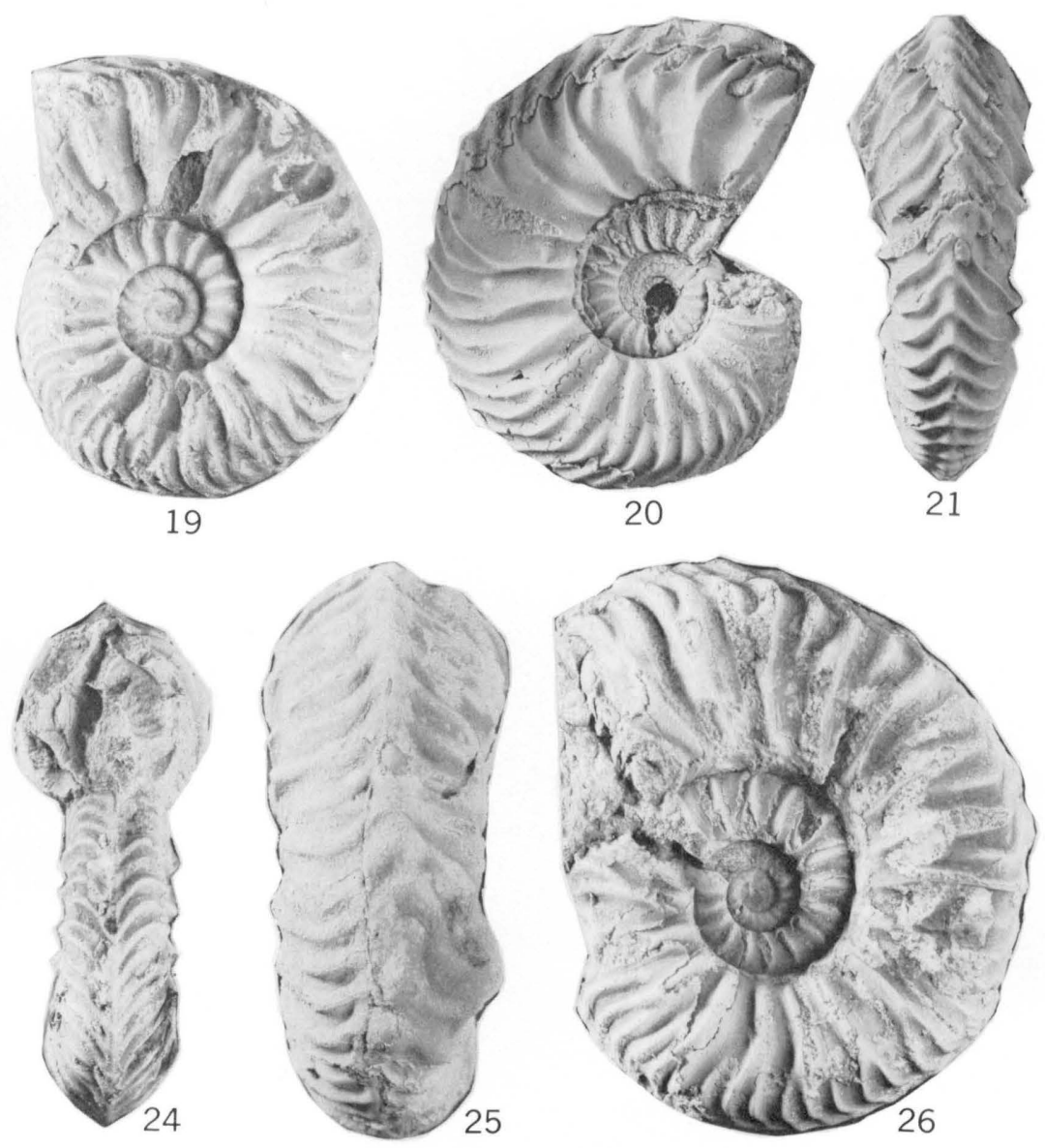

CARDIOCERAS (VERTEBRICERAS), GROSSOUVRIA?, AND GROSSOUVRIA (POCULISPHINCTES?) 


\section{PLATE 26}

[All figures natural size unless otherwise indicated]

FIGURES 1-7, 11-13. Cardioceras (Sagitticeras) obtusum Reeside (p. 40)

1, 2. Paratype, USNM 32352, from USGS Mesozoic loc. 16615.

3, 4. Hypotype, USNM 303717, from USGS Mesozoic loc. 16615.

5-7. Hypotype, USNM 303718, from USGS Mesozoic loc. 6283. Figures 5 and 6 show different parts of the venter.

11. Hypotype, USNM 303719, from USGS Mesozoic loc. 29677.

12, 13. Holotype, USNM 32351, from USGS Mesozoic loc. 16616.

8-10. Prososphinctes? sp. (p. 40)

Figured specimens, USNM 303722, from USGS Mesozoic loc. 19579. Figures 8 and $10(\times 2)$.

14-33. Cardioceras (Subvertebriceras) canadense Whiteaves (p. 38)

14, 15. Hypotype, USMN 32343, from USGS Mesozoic loc. 16620.

16, 17. Hypotype, USNM 32342, from USGS Mesozoic loc. 16620.

18, 19. Hypotype, USNM 303709, from USGS Mesozoic loc. 16620.

20, 21. Hypotype, USNM 303708, from USGS Mesozoic loc. 29769.

22, 23. Hypotype, USNM 303705, from USGS Mesozoic loc. 29769.

24. Hypotype, USNM 303706, from USGS Mesozoic loc. 29769.

25, 26. Hypotype, USNM 303707, from USGS Mesozoic loc. 29769.

27, 28. Hypotype, USNM 32341, from USGS Mesozoic loc. 16620.

29, 31. Hypotype, USNM 303704, from USGS Mesozoic loc. 29769.

30. Hypotype, USNM 303703, from USGS Mesozoic loc. 29769.

32, 33. Hypotype, USNM 303702, from USGS Mesozoic loc. 30087. 

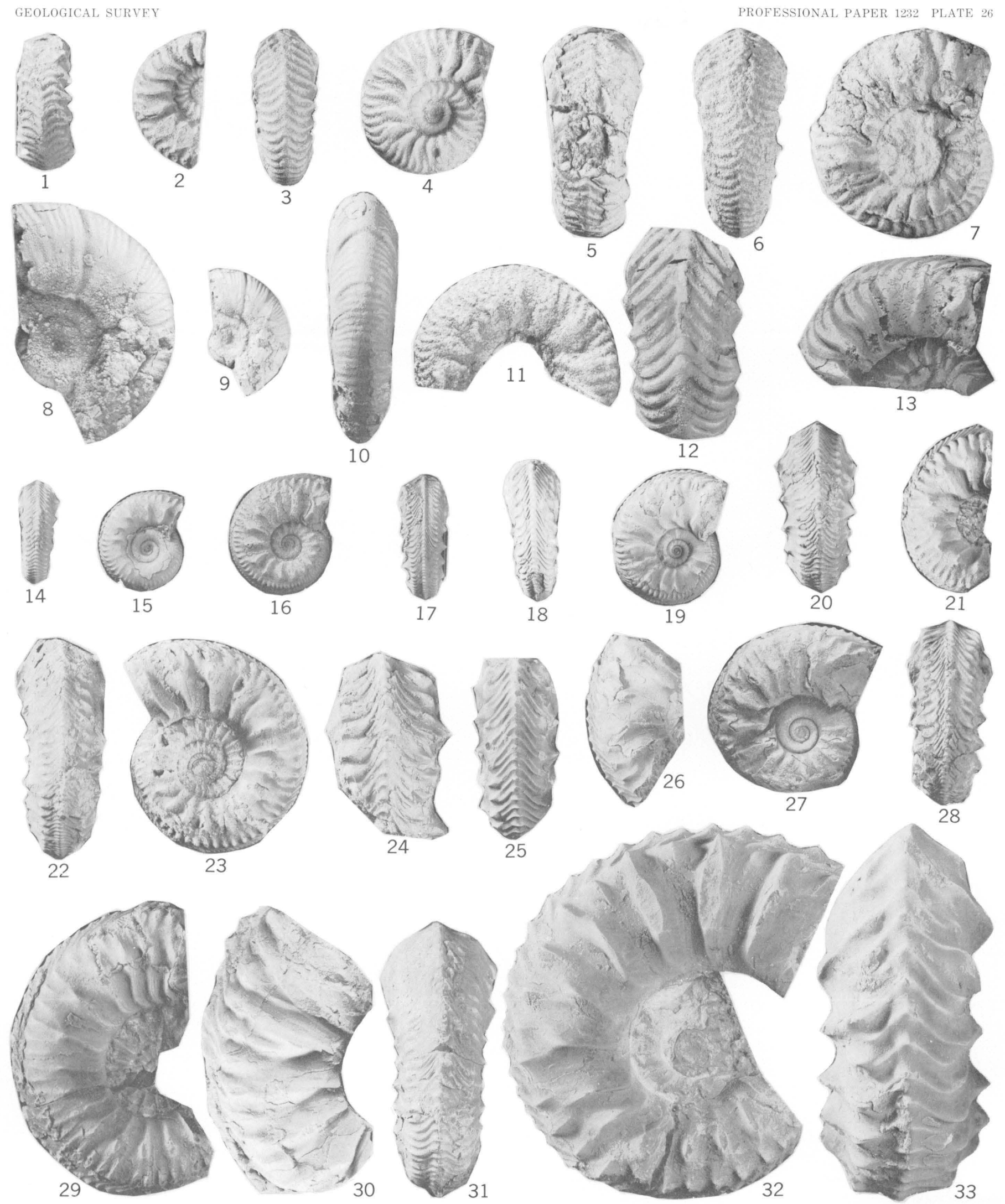

CARDIOCERAS (SAGITTICERAS), PROSOSPHINCTES?, AND CARDIOCERAS (SUBVERTEBRICERAS) 